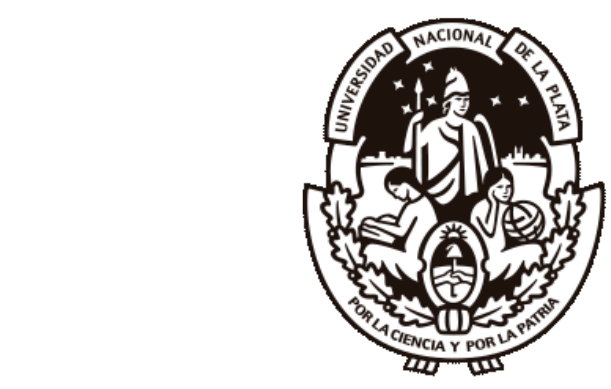

Universidad Nacional De LA Plata

Facultad de Ciencias Exactas

Departamento de Física

Tesis presentada para optar al grado de Doctor de la Facultad de Ciencias Exactas

\title{
ESCALAS ESPACIALES Y MECANISMOS DE RELAJACIÓN EN LÍQUIDOS SOBREENFRIADOS
}

Tesista: Lic. Alejandro Seif

Director: Dr. Tomas S. Grigera 



\section{Resumen}

Tanto en el mundo natural como en el artificial, existen sistemas compuestos únicamente por elementos simples para los que se observan fenómenos de gran complejidad.

Nuestro objetivo fue estudiar estos sistemas, mediante modelos lo más sencillos posibles en su formulación, pero que a la vez sean capaces de exhibir un comportamiento complejo. Pusimos nuestro énfasis en fenómenos para los que el sistema comienza a salirse del equilibrio (líquidos sobre-enfriados) y fenómenos en que el sistema se encuentra completamente fuera de equilibrio (partículas autopropulsadas)

En el capítulo 2 introdujimos los antecedentes y conceptos relacionados al estudio de la transición vítrea para líquidos sobreenfriados. Se presentaron las características salientes de vidrios, líquidos sobreenfriados y cristales, haciendo hincapié en descripciones de la estructura de estos. En el capítulo 3 presentamos el método Monte Carlo Cinético y nociones técnicas para modelos formadores de vidrios. Luego, en el capítulo 4 presentamos los resultados obtenidos para el modelo de retículo PCTCC. Mostramos un estudio de metaestabilidad y una posible temperatura de Kauzmann $T_{K}$. Utilizando el método de tiempos cortos, determinamos una temperatura espinodal termodinámica. Finalmente presentamos un estudio de envejecimiento mediante un exponente de escala para los tiempos de espera. En el capítulo 5, caracterizamos el modelo de retículo t154, e investigamos su dinámica tanto en condiciones de contorno periódicas, así como utilizando una cavidad que lo confina. Realizamos un estudio de su estática y extraemos una longitud de correlación estática.

En el capítulo 6 introdujimos los antecedentes y conceptos relacionados al novedoso campo de la materia auto-propulsada. En el capítulo 7, discutimos el uso de Dinámica Molecular y el estudio de efectos de tamaño finito mediante el método de sub-bloques. Los capítulos 8 y 9 presentan los resultados hallados para mezclas binarias de partículas auto-propulsadas con volumen excluido y los efectos de agregar interacciones orientacionales en todas ellas (Capitulo 9). Notamos que las partículas únicamente se diferencian en su velocidad, clasificándolas en rápidas y lentas. Para el caso sin interacción orientacional observamos una transición de segregación al ralentizar a las partículas lentas, detectando presencia de estructura cristalina mediante medidas de funciones de correlación estática. Corroboramos la presencia o ausencia de esta transición mediante un análisis de sub-bloques para un estudio de tamaño finito. Cuando incorporamos interacciones orientacionales, el sistema presenta segregación en una región de parametros distinta al caso anterior y sin indicios de estructura de largo alcance.

Finalmente, presentamos una discusión y conclusiones del trabajo de tesis en el capítulo 10. 


\section{Agradecimientos}

- A mi director Tomás S. Grigera, siempre paciente con mis caprichos.

- A Gabriel Baglietto, un colaborador excepcional.

- Al IFLYSIB y la gente con que compartí trabajo, comida, jornadas y tareas de carpintería.

- Al INIFTA y la gente que estuvo cerca en los primeros pasos en la investigación.

- Al CONICET y a AGENCIA por la financiación de mi vida de graduado y gastos de mi doctorado.

- A mis padres por la financiación de mi vida de pre-graduado y otros servicios.

- A mis amigos. Variados, todos me enseñaron cosas.

- A mi esposa Arika, que siempre busca sacar lo mejor de mi.

La realización de esta tesis fue posible, debido a una beca doctoral de CONICET y a fondos de AGENCIA. Tuve lugar de trabajo en el INIFTA durante 2013-2015 y luego en el IFLYSIB 2015-2017. 


\section{Índice general}

$\begin{array}{lr}\text { Resumen } & 1\end{array}$

$\begin{array}{lr}\text { Agradecimientos } & 2\end{array}$

$\begin{array}{lr}\text { 1. Introducción General } & 9\end{array}$

I Sistemas Vítreos 12

2. Introducción a los Líquidos Sobreenfriados 13

2.1. Vidrios: Un Problema muy Viejo y muy Vigente . . . . . . . . . . . . . 13

2.2. Vidrios . . . . . . . . . . . . . . . . . . 15

2.2.1. Materia Condensada en una Carilla . . . . . . . . . . . 15

2.2.2. Rigidez de un Vidrio . . . . . . . . . . . . . . . . . . 16

2.3. Fenomenología Vítrea . . . . . . . . . . . . . . . . . . . . 17

2.3.1. El Desorden de los Vidrios . . . . . . . . . . . . . . . . . . 18

2.3.2. Líquidos Formadores de Vidrios . . . . . . . . . . . . . . . . 18

2.3.3. La Teoría de Adam Gibbs . . . . . . . . . . . . . . . . . . 19

2.3.4. Crisis de Entropía y Tiempo de Relajación . . . . . . . . . . 20

2.3.5. Difusión y la Relación de Stokes-Einstein . . . . . . . . . . . 21

2.3.6. Relajación en Dos Pasos . . . . . . . . . . . . . . . . . . 21

2.3.7. La Jaula . . . . . . . . . . . . . . . . . . . . . . . . 22

2.3.8. Caída de $c_{p} \ldots \ldots \ldots \ldots \ldots \ldots \ldots$

2.3.9. La Exponencial Estirada . . . . . . . . . . . . . . . . . . 24

2.3.10. Correlaciones Dinámicas y Envejecimiento . . . . . . . . . . 25

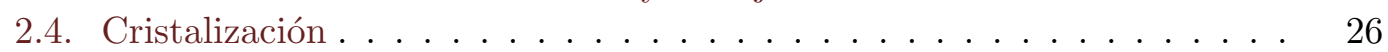

2.4.1. Nucleación . . . . . . . . . . . . . . . . . . . . . 26

2.4.2. Dinámica de la Nucleación . . . . . . . . . . . . . . . . . . . . . . . 27

2.4.3. La Temperatura Espinodal Cinética . . . . . . . . . . . . 28

2.4.4. Formación de Cristales . . . . . . . . . . . . . . . . 30

2.4.5. La Función de Correlación de Pares g(r) . . . . . . . . . . 30

2.4.6. El Factor de Estructura Estático $\mathrm{S}(\mathrm{k}) \ldots \ldots \ldots$

2.5. Correlación Punto Conjunto . . . . . . . . . . . . . . . . . 33

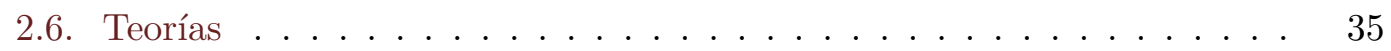

2.6.1. Topografía: Propuesta de Paisaje de Energía . . . . . . . . . . 35 
2.6.2. Entonces, ¿Cómo Detectamos un Vidrio? . . . . . . . . . . . 35

2.6.3. Teoría de Modos acoplados MCT . . . . . . . . . . . 36

2.6.4. Transición aleatoria de primer orden RFOT (Teoría de Mosaico) 37

2.7. Modelos de Vidrio de Retículo . . . . . . . . . . . . . . . . . . . . 39

2.8. Objetivos de Este Trabajo . . . . . . . . . . . . . . . . . . 40

3. Simulaciones I: Metodología Utilizada en el Estudio Modelos de Retículo para Líquidos Sobreenfriados $\quad 42$

3.1. Simulaciones Computacionales . . . . . . . . . . . . . . . . . . . 42

3.2. Sistemas Discretos : Retículos . . . . . . . . . . . . . . 43

3.3. El Método Monte Carlo MC . . . . . . . . . . . . . . . . . . . 43

3.3.1. Muestreo de Importancia . . . . . . . . . . . . . . . . . . . . . 44

3.3.2. Procesos de Markov . . . . . . . . . . . . . . . . . . 45

3.3.3. Ergodicidad y Balance Detallado . . . . . . . . . . . . . 45

3.3.4. El Algoritmo de Metropolis . . . . . . . . . . . . . . . . . 46

3.4. El Método Monte Carlo Cinético (KMC) . . . . . . . . . . . . . . 46

3.4.1. Sistemas de Eventos Improbables y Perdida de Ergodicidad . . . 47

3.5. Herramientas Complementarias . . . . . . . . . . . . . . . . . . 49

3.5.1. Dinámica de Tiempos Cortos . . . . . . . . . . . . . . 49

3.5.2. Prueba BIC . . . . . . . . . . . . . . . . 50

4. Modelo PCTCC

4.1. Modelo PCTCC . . . . . . . . . . . . . . . . . . . . . . . . 54

4.2. Comparación entre Monte Carlo Metropolis y Monte Carlo Cinético . . 56

4.3. Estudio de Metaestabilidad . . . . . . . . . . . . . . . . . . . 57

4.4. Límites de la Metaestabilidad . . . . . . . . . . . . . . . . . . 60

4.5. Dinámica de Tiempos Cortos . . . . . . . . . . . . . . . 61

4.6. Estudios de Envejecimiento . . . . . . . . . . . . . . . . . 64

5. Modelo t154

5.1. El Modelo de Biroli Mezard . . . . . . . . . . . . . . . . . . 68

5.2. t154: Una Proporción que no Cristaliza . . . . . . . . . . . . . 70

5.3. Dinámica . . . . . . . . . . . . . . . . . . . . . 72

5.3.1. Efectos de Tamaño Finito . . . . . . . . . . . . . . . . 74

5.4. Estructura . . . . . . . . . . . . . . . . . . . 77

II Partículas Autopropulsadas $\quad 86$

6. Introducción a la Materia Activa $\quad \mathbf{8 7}$

6.0.1. La Imposibilidad de una Descripción Teórica General para Materia Activa . . . . . . . . . . . . . . . 88

6.0.2. Bandadas. . . . . . . . . . . . . . . . . . . 89

6.1. Transiciones de Fase fuera del Equilibrio . . . . . . . . . . . . . . . . 90

6.2. Complejidad y Fenómenos Emergentes . . . . . . . . . . . . . . . . 91

6.3. Fuerzas Intermoleculares y Potenciales Modelo . . . . . . . . . . . . . . 92

6.4. Interacciones Orentacionales . . . . . . . . . . . . . . . . 95

6.4.1. El Modelo XY . . . . . . . . . . . . . . . . . . . . . . 95 
6.4.2. El Modelo de Vicsek . . . . . . . . . . . . . . . . . . . . . 95

6.5. Objetivos de este trabajo . . . . . . . . . . . . . . . . . . 96

7. Simulaciones II: Metodología Utilizada en el Estudio de Materia Ac$\begin{array}{ll}\text { tiva } & \mathbf{9 8}\end{array}$

7.1. Sistemas Continuos . . . . . . . . . . . . . . . . . . . . 98

7.2. Dinámica Molecular . . . . . . . . . . . . . . . . . . . . . . . 99

7.2.1. Integración de las Ecuaciones de Movimiento . . . . . . . . . . 99

7.2.2. Relación con la Mecánica Estadística . . . . . . . . . . . . . . 100

7.3. Detalles de optimización en simulaciones DM . . . . . . . . . . . . 101

7.3.1. Potencial Lennard-Jones truncado . . . . . . . . . . . . . . . . . 101

7.3.2. Paso temporal de las simulaciones . . . . . . . . . . . . . . . 102

7.4. Efectos de Tamaño Finito . . . . . . . . . . . . . . . . . . . . . 102

7.4.1. Cumulante de Binder . . . . . . . . . . . . . . . . . . . . . 103

7.4.2. El Análisis de Sub-Bloques como Medida de Efectos de Tamaño Finito . . . . . . . . . . . . . . . . . . . . 104

7.4.3. Muestreo Utilizando Bloques . . . . . . . . . . . . . . 105

8. Resultados para Partículas Auto-Propulsadas con Volumen Excluído107

8.1. El modelo . . . . . . . . . . . . . . . . . . . . . . . 107

8.2. El Espacio de Parámetros Utilizado . . . . . . . . . . . . . . . . . . 108

8.3. Determinación de comportamiento estacionario . . . . . . . . . . . . 109

8.4. Clasificación de Estados Estacionarios Según $V_{S}$. . . . . . . . . . . . . 112

8.5. Distribuciones Locales de Densidad y Concentración . . . . . . . . . . . 114

8.6. Transición de segregación y cumulantes de Binder . . . . . . . . . . 116

8.7. Detección de Orden Mediante $S(k)$ y g(r) . . . . . . . . . . . . 118

9. Resultados para Partículas Auto-Propulsadas con Volumen Excluido e Interacción Orientacional $\quad 123$

9.1. Interacción Lennard Jones Junto con Interacción Orientacional . . . . . 123

9.1.1. Integración de las Ecuaciones de Movimiento Considerando una Interacción Orientacional . . . . . . . . . . . . . . . . 123

9.2. Determinación y Clasificación de Estados Estacionarios . . . . . . . . . 124

9.3. Distribuciones Locales de Densidad y Concentración . . . . . . . . . 127

9.4. Transición de Segregación y Cumulantes de Binder . . . . . . . . . . 128

9.5. Detección de orden mediante $\mathrm{S}(\mathrm{k})$ y $\mathrm{g}(\mathrm{r}) \ldots \ldots \ldots$

$\begin{array}{lr}\text { III Conclusiones Generales } & 134\end{array}$

$\begin{array}{ll}\text { 10.Conclusiones } & 135\end{array}$

10.1. Modelo PCTCC . . . . . . . . . . . . . . . . . . . 135

10.2. Modelo t154 . . . . . . . . . . . . . . . . . . . 136

10.3. Partículas Auto-Propulsadas con Volumen Excluído . . . . . . . . . . 137

10.4. Partículas Auto-Propulsadas con Volumen Excluído e Interacción Orientacional . . . . . . . . . . . . . . . . . . . . 138

10.5. Conclusiones Generales . . . . . . . . . . . . . . . . . 138 
Bibliografía 140 


\section{Índice de figuras}

2.1. Esquema de la relajación en dos pasos . . . . . . . . . . . . . . . 22

2.2. Esquema de temperatura espinodal cinética $T_{S P} \ldots \ldots \ldots$. . . . . . . . 29

2.3. Ejemplos esquemáticos para función de correlación de pares $\mathrm{g}(\mathrm{r}) \ldots$. . 31

2.4. Caricatura de un paisaje de energía para un sistema en el continuo. . . 35

3.1. Representación de la lógica para la evolución del algoritmo Monte Carlo Cinético KMC . . . . . . . . . . . . . . . . . . . . . 48

3.2. Caricatura de la prueba de condición inicial BIC . . . . . . . . . . 51

4.1. Caricatura del modelo de retículo PCTCC para un caso bidimensional 54

4.2. Comparación entre el tiempo real de simulación entre simulaciones Monte Carlo Metropolis (MMC) y Monte Carlo Cinético (KMC). . . . . . . 57

4.3. Enfriamiento a distintos ritmos del modelo .PCTCC . . . . . . . . . . . . 58

4.4. Gráfico Angell mostrando un comportamiento no-Arrhenius. . . . . . . 59

4.5. Densidad $\rho$ en función del tiempo. . . . . . . . . . . . . . . . 60

4.6. Masa cristalina $m$ en función del tiempo. . . . . . . . . . . . . . . 61

4.7. Evolución de la masa cristalina m comenzando desde el desorden inicial 63

4.8. Evolución de la masa cristalina $m$ usando dinámica de tiempos cortos STD ............................... 63

4.9. Medidas de autosolapamiento para $T=\mu / 9$ mostrando envejecimiento. $\quad 65$

4.10. Ajuste sigmoideal para determinar relación entre tiempos de espera y

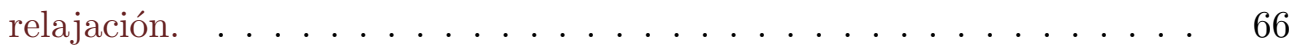

5.1. Caricatura del modelo de Biroli Mézard para un caso bidimensional . . 70

5.2. Ajuste VFT para dinámicas $\mathrm{C} / \mathrm{GC} \ldots \ldots \ldots . \ldots . \ldots 73$

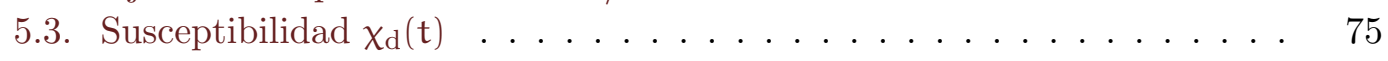

5.4. Tiempo de relajación $\tau$ vs $\rho$ usando condiciones de contorno periódicas (PBCs) Canónico . . . . . . . . . . . . . . . . . . 76

5.5. Tiempo de relajación $\tau$ vs $\langle\rho\rangle$ usando PBCs GC . . . . . . . . . . 76

5.6. Tiempo de relajación punto-conjunto (PTS) $\tau$ vs $\langle\rho\rangle$ usando condiciones de contorno amorfas (ABCs) gran canónica (GC) . . . . . . . . 77

5.7. Densidad $\rho$ vs $\alpha$ determinados para $L=30 \ldots \ldots \ldots 78$

5.8. Densidad $\rho$ vs $\alpha_{1}$ para dinámica PBCs GC. . . . . . . . . . . . 78

5.9. Fluctuaciones de la densidad para dinámicas GC en condiciones $\mathrm{ABCs}$ y $\mathrm{PBC} \ldots \ldots \ldots \ldots \ldots \ldots \ldots \ldots \ldots$

5.10. Medidas de autosolapamiento global $Q(t)$ y punto conjunto $q(t) \ldots . \quad 81$

5.11. Prueba BIC para cavidades de distintos tamaños. . . . . . . . . . . . 82 
5.12. Ajustes de las predicciones teóricas sobre los valores asintóticos $\mathrm{q}_{\infty} \mathrm{y} \mathrm{Q}_{\infty} \cdot 84$

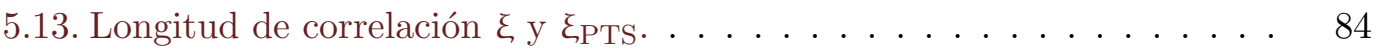

5.14. Ley de potencia para tiempos de relajación en función de longitud de

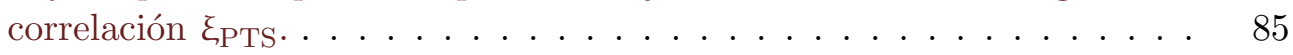

6.1. Representación esquemática para potenciales intermoleculares. . . . . . 94

6.2. Representación de los rangos de las interacciones entre partículas. . . . 97

7.1. El potencial Lennard Jones utilizado, truncado para $r_{c}=2^{1 / 6} \sigma \quad \ldots$.

7.2. Representación del análisis de sub-bloques . . . . . . . . . . . . . 106

8.1. Distintas configuraciones iniciales con el mismo conjunto de parámetros 110

8.2. Configuraciones finales con el mismo conjunto de parámetros . . . . . . 111

8.3. Prueba de condición inicial usando observable LJSF . . . . . . . . . . . 111

8.4. Configuraciones finales para distintos valores de $V_{S} \ldots \ldots \ldots$

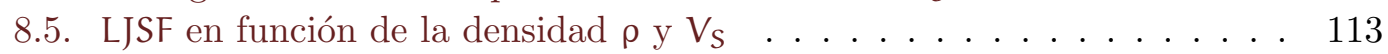

8.6. Histograma de densidad local $\rho_{\mathrm{B}}$. . . . . . . . . . . . . . . . 115

8.7. Ajuste gaussiano para determinar las densidades mas prominentes en el histograma de densidades locales $\rho_{B} \ldots \ldots \ldots \ldots \ldots \ldots$

8.8. Ley de potencia para diferencia de densidad local en función de $\mathrm{V}_{\mathrm{S}}$. . 116

8.9. Histograma para la concentración de partículas rapidas $\mathrm{C}^{\mathrm{F}}$. . . . . 117

8.10. Cumulante de Binder para concentración de partículas rápidas $U\left(C^{f}\right) \cdot 117$

8.11. Cumulantes de Binder para concentración de partículas rápidas $U\left(C^{f}\right)$, en función de $V_{S}$ para distintos valores de FPR. . . . . . . . . . . . 118

8.12. Función de correlación de pares g(r) para todas las partículas. . . . . . 119

8.13. Función de correlación de pares $\mathrm{g}(\mathrm{r})$ discriminando por tipo de partícula. 120

8.14. Factor de estructura estático $S(k)$ para todas las partículas. . . . . . . 120

8.15. Factor de estructura estático $S(k)$ discriminando por tipo de partícula. 121

8.16. Diagrama de fase cualitativo. . . . . . . . . . . . . . . . 122

9.1. Prueba de condición inicial usando los observables LJSF y $\Phi$. . . . . . 125

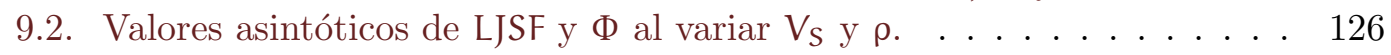

9.3. Configuraciones finales del sistema incorporando interacciones orientacionales. . . . . . . . . . . . . . . . . . . 126

9.4. Histograma de la densidad local $\rho_{\mathrm{B}} \ldots \ldots \ldots \ldots$. . . . . . . . . . 127

9.5. Histograma de la concentración local de partículas rápidas $C^{F} \ldots \ldots$

9.6. Cumulante de concentración de partículas rápidas $\mathrm{U}\left(\mathrm{C}^{\mathrm{F}}\right)$. . . . . . . 129

9.7. Función de correlación de pares $\mathrm{g}(\mathrm{r})$ para todas las partículas, incorporando interacción orientacional. . . . . . . . . . . . . . .

9.8. Función de correlación de pares $\mathrm{g}(\mathrm{r})$ discriminando por tipo de partícula, incorporando interacción orientacional. . . . . . . . . . . . .

9.9. Factor de estructura estático $S(k)$ para todas las partículas, considerando interacción orientacional. . . . . . . . . . . . . . . . .

9.10. Factor de estructura estático $S(k)$ discriminando por tipo de partícula, considerando interacción orientacional. . . . . . . . . . . . . . . 132

9.11. Diagrama de fase cualitativo considerando interacción orientacional. . . 133 


\section{Capítulo 1}

\section{Introducción General}

Existe una buena comprensión de los sistemas en equilibrio. La física de materia condensada nos brinda herramientas para describir y determinar lo que le sucede a grandes números de partículas simples que interactúan mediante fuerzas conocidas. El comportamiento de equilibrio puede ser extraído de la minimización de la energía libre F, cuya obtención depende de la función de partición Z. Si bien el cálculo de Z puede presentar un problema matemático/computacional muy difícil, al menos, sabemos que existe una receta general disponible para estudiar el equilibrio.

La situación es distinta cuando deseamos estudiar el sistema cuando éste está fuera del equilibrio. Un sistema líquido enfriado muy por debajo de su temperatura de fusión puede salirse del equilibrio por aumento exponencial del tiempo de relajación $\tau$ (transición vítrea). Otro caso son los sistemas de partículas autopropulsadas, las cuales son sistemas de agentes autónomos capaces de convertir energía del entorno en movimiento persistente. Estas partículas están manifiestamente fuera del equilibrio, precisamente por su consumo de energía en el movimiento. Estos dos casos son ejemplos donde ya no contamos con recetas sobre como extraer información del sistema.

Las propiedades macroscópicas de sistemas de materia condensada generalmente dependen fuertemente de interacciones complejas entre los constituyentes microscópicos del sistema. Podemos obtener una comprensión teórica estudiando analítica o numéricamente un modelo simplificado del sistema real, donde el comportamiento predicho depende de los parámetros del modelo específico considerado. Sin embargo, existen casos donde el comportamiento colectivo que emerge es en gran parte independiente de los detalles microscópicos del sistema y, como consecuencia de esto, es independiente del modelo utilizado para describir el fenómeno. Esta propiedad se conoce como universalidad [Calabrese and Gambassi, 2005] y caracteriza el comportamiento físico cuando el sistema se acerca al punto crítico. Allí, el sistema atraviesa una transición de fase 
continua. La emergencia del comportamiento colectivo es revelada por el crecimiento de una longitud de correlación $\xi$, definida como la distancia típica a la que las variables microscópicas estan correlacionadas. Lejos del punto crítico, ६ es típicamente del orden de las interacciones microscópicas, pero a medida que el sistema se aproxima al punto crítico, $\xi$ crece hasta diverger. De esta manera, posicionándonos cerca del punto de transición, $\xi$ se vuelve mesoscópica y provee una escala de longitud relevante para el sistema critico.

En los líquidos sobreenfriados, la intuición física indica que el crecimiento del tiempo de relajación estructural (esencialmente proporcional a la viscosidad) debe estar relacionado al crecimiento de alguna longitud de correlación そ [Cavagna, 2009], como también se ha concluido recientemente desde un enfoque riguroso [Montanari and Semerjian, 2006a]. Sin embargo, el carácter amorfo del sistema hace difícil detectar correlaciones al no conocerse el parámetro de orden (las correlaciones de densidad de dos partículas, por ejemplo, no sufren ningún cambio dramático). Por estos motivos, la búsqueda de una longitud de correlación creciente ha sido el objetivo de muchos estudios en los últimos 20 años [Berthier and Biroli, 2011].

De acuerdo al tiempo característico del experimento, los líquidos sobreenfriados pueden ser observados como tales (es decir en equilibrio), o bien como vidrios, es decir fuera del equilibrio. Dada la velocidad de crecimiento de los tiempos de relajación al bajar la temperatura, estos sistemas típicamente se observan (si la temperatura es menor de aproximadamente un tercio de la temperatura de fusión) en un estado no ergódico, de viscosidad extremadamente alta y con las propiedades mecánicas de un sólido pero estructura amorfa. Este es el estado vítreo.

Recientemente ha comenzado a estudiarse con mucho interés otro tipos de sistemas, inherentemente fuera del equilibrio debido a estar sometidos a permanente inyección de energía: son los sistemas llamados de materia activa [Ramaswamy, 2010], que han sido motivo de numerosos estudios mediante herramientas de la materia condensada. Comparten con los sistemas vítreos el estar apartados del equilibrio, aunque por motivos distintos (ruptura de ergodicidad vs. inyección de energía).

Es notorio que si bien no hay equilibrio, los sistemas de materia activa compuestos por partículas auto-propulsadas, son capaces de alcanzar estados estacionarios y sufrir transiciones de fase fuera del equilibrio. Estos sistemas [Vicsek and Zafeiris, 2012], aún sin interacciones orientacionales, exhiben una fenomenología rica, presentando transiciones de segregación.

Esto presenta una oportunidad para estudiar sistemas próximos al no-equilibrio (líquidos sobreenfriados) y sistemas completamente fuera de equilibrio (partículas auto- 
propulsadas) desde el punto de vista de su dinámica y organización, fuera del equilibrio termodinámico.

El objetivo general de este trabajo de tesis es estudiar la relación entre las escalas de longitud de correlación y la dinámica de sistemas de líquidos formadores de vidrios, así como el surgimiento de correlaciones espaciales y transiciones de fase fuera del equilibrio en sistemas de partículas autopropulsadas.

Los aportes originales específicos que realiza este trabajo de tesis son:

- La caracterización de la metaestabilidad y el envejecimiento, para el modelo de retículo PCTCC de líquido formador de vidrio, así como la presentación de los beneficios en performance del uso del algoritmo Monte Carlo Cinético KMC, respecto al algoritmo Monte Carlo Metropolis MMC.

- Re-visitar el estudio del modelo de retículo t154 para líquido formador de vidrio, realizando un estudio de su dinámica y estructura en el contexto de condiciones de contorno periódicas (PBC) así como condiciones de contorno amorfas (ABC).

- La extracción de una longitud de correlación estática そ para el modelo t154, que crece cuando el sistema aumenta su densidad.

- La verificación de que ambos modelos de retículo presentan características de fenomenología vítrea, pero su comportamiento en altas densidades parece alejarse del de un líquido sobreenfriado.

- La determinación de que la diferencia de velocidades y la densidad controlan la segregación de una mezcla binaria de partículas auto-propulsadas (con y sin interacción orientacional). Hallamos la existencia de una transición de fase de segundo orden para el caso no orientacional, así como una región de criticalidad cuando la interacción orientacional esta activada.

- La observación de que la interacción orientacional inhibe la formación de orden estructural de largo alcance. Este orden está presente para una región específica de parámetros, cuando la interacción orientacional se encuentra desactivada. Notamos que el agregado de la interacción orientacional genera un orden de corto alcance variable para todo el espacio de parámetros, que no fue observado en el caso sin interacciones orientacionales. 


\section{Parte I}

\section{Sistemas Vítreos}




\section{Capítulo 2}

\section{Introducción a los Líquidos Sobreenfriados}

\subsection{Vidrios: Un Problema muy Viejo y muy Vigente}

Cuerdas multidimensionales, agujeros negros y energía oscura reciben gran atención de la prensa y el público en general. Se trata de fenómenos exóticos, excitantes y hasta incluso místicos para algunos. También resultan completamente alejados de la vida cotidiana. Bien puede ser que nuestros propios cuerpos estén compuestos de excitaciones que oscilan como una cuerda, o que los agujeros negros gobiernen el pasado y futuro del universo, pero para la mayoría de las personas, el impacto de éstos en nuestra vida diaria es nulo.

No está mal recordar que hace un par de siglos, la electricidad o el magnetismo, no jugaban rol conocido alguno para la vida diaria de la gente. Hoy en día nadie se sorprende por tocar una tecla e iluminar una habitación oscura, e incluso podemos enfadarnos si al realizar una videollamada, con alguien a miles de kilómetros de distancia desde un dispositivo que entra en un bolsillo, la calidad de la imagen no es alta definición. Pero un par de siglos atrás, estos fenómenos que hoy nos parecen cotidianos, eran esotéricos, curiosidades de científicos e intelectuales trabajando en laboratorios aislados. Como quizás lo son hoy para muchos las cuerdas o los agujeros negros.

En el mundo industrializado y globalizado en el que vivimos, la vida y bienestar de la mayoría, depende completamente de los descubrimientos realizados por científicos considerados esotéricos un par de siglos atrás. El electromagnetismo y la termodinámica de los sólidos, líquidos y gases, luego acompañada por la mecánica cuántica, la comprensión moderna de los átomos y moléculas y como se organizan, todo confluye en lo que hoy conocemos como física de la materia condensada. 
Pero entre los problemas que se fueron presentando y comprendiendo a lo largo de de la historia reciente de la ciencia, hubo uno que fue quedando inconcluso con el paso de los siglos. Y no fue por falta de intentos o ideas innovadoras que aún hoy no exista una teoría, al menos rudimentaria, que nos explique que esta sucediendo. Nos referimos al problema de los vidrios.

Ya los antiguos egipcios, hace aproximadamente 3500 años [Varberg et al., 2015], utilizaban técnicas de fabricación de vidrio, fabricando objetos de distinta opacidad, color y forma. Estas técnicas fueron copiadas, mejoradas, olvidadas y re descubiertas por distintos vidrieros alrededor del mundo a lo largo de la historia de la humanidad. Los vidrios, y los líquidos sobreenfriados con los que usualmente son preparados, han sido objeto de estudio por casi 100 años. Un caso notable es el llamado experimento mas largo del mundo o caída de la gota de brea [Edgeworth et al., 1984], que desde 1927 estudia el flujo de brea en un embudo, exhibiendo de manera sencilla la existencia de sustancias que parecen sólidas, pero sin embargo fluyen con una viscosidad muy alta. Se estima que la viscosidad de la brea $\eta_{\mathrm{Brea}} \simeq 2 \times 10^{9}$ Poise, sin embargo se consideran vidrios a materiales con viscosidad $\eta>10^{13}$ Poise.

Actualmente, los vidrios juegan un rol clave en materia de tecnología, tanto actual como en desarrollo [Ediger and Harrowell, 2012]. Fibra óptica, semiconductores y polímeros, son algunas de las tecnologías que cambian la manera en que funciona el mundo gracias a sus aplicaciones. Sin embargo, todo estos años de uso y experimentación aún no han permitido que se pueda encontrar una teoría que responda preguntas fundamentales al respecto de su comportamiento.

Uno de los numerosos frutos de la llegada de las simulaciones computacionales, han sido los avances en la comprensión de estos materiales amorfos. Mediante simulaciones, podemos contemplar tanto modelos atomísticos como modelos mínimos (en la literatura llamados toy-models) que intentan dar cuenta de la fenomenología de estos materiales de maneras inaccesibles para experimentos y así contrastar con algunas de las diversas teorías propuestas.

En este trabajo se estudiarán mediante simulaciones computacionales, dos modelos mínimos que denotaremos como PCTCC y t154, que aún en su sencillez y con sus severas limitaciones, describen algunos aspectos de la fenomenología vítrea. 


\section{2. $\quad$ Vidrios}

\subsubsection{Materia Condensada en una Carilla}

Si tuviésemos que resumir toda la mecánica estadística en unas pocas ideas, podemos pensar a un sistema en términos de probabilidades de configuraciones [Stein and Newman, 2013]. Dejemos fijas cantidades como el número de partículas (cerca de 1023), volumen, tipo de partículas, etc y tomemos como parámetro externo a la temperatura T. La temperatura $T$ actúa como una especie de selector de probabilidades para indicarnos cuales configuraciones de los grados de libertad estarán presentes. A medida que T aumenta, aparecen configuraciones con mayor energía E. De esto exactamente se trata la distribución de Boltzmann, donde $\mathrm{k}_{\mathrm{B}}$ es el factor de Boltzmann y la probabilidad relativa de que aparezca una configuración con energía $E$ a temperatura $T$, viene dada por:

$$
\exp \frac{-E}{k_{B} T}
$$

Esto también significa, que configuraciones con energía $E$ mas alta que $k_{B} T$ son fuertemente suprimidas. Otro factor, que esta expresión no tiene en cuenta, es que a mayor E, existe una mayor cantidad de configuraciones diferentes, todas con energía E. La cantidad de configuraciones distintas con una misma energía E es una medida de la entropía $S$ del sistema. De aquí concluimos, que mientras mas alta sea la cantidad de configuraciones posibles para una dada E, más alta será la entropía S. Y como en general, mientras más alta la temperatura T mas alta la energía E, más alta será la entropía $S$ del estado termodinámico resultante.

La idea de un numero creciente de configuraciones con el aumento de temperatura resulta natural si pensamos en todas las maneras que los átomos se acomodan en un líquido o, más aún, en un gas. Ambos son estados estados desordenados y de alta entropía, siendo la entropía del gas mayor a la del líquido. Ahora, a medida que la temperatura es disminuída, las configuraciones desordenadas dejan de ser seleccionadas por el sistema y las únicas configuraciones que se vuelven probables son unas pocas y de baja energía.

Pero uno podria preguntarse: ¿cómo medir o cuantificar estos ordenamientos o desordenamientos? Así es como los parámetros de orden, nuevas variables termodinámicas, entran en juego. Se trata de cantidades que podemos definir para un sistema, cuya cualidad es ser cero en una fase (alta temperatura por ejemplo) y no nula, para otra fase (baja temperatura). En algunos sistemas, el parámetro de orden se vuelve no nulo de manera discontinua (pegando un salto), en otras crece continuamente desde cero (pero 
su derivada es discontinua). Un ejemplo de esto, sea $S$ la entropía, es el calor especifico $C_{V}=\left.T \frac{\partial S}{\partial T}\right|_{V}$, que según la naturaleza del material estudiado y su transición, puede tener un crecimiento continuo o discontinuo.

Los parámetros de orden, no solo muchas veces nos brindan una ruta experimental para encontrar transiciones de fase, si no que además son centrales para entender la física de la materia condensada.

No podemos dejar de resaltar, que este análisis, las técnicas matemáticas y los problemas resueltos usando estas técnicas, resultan potentes para material ordenados. Para estos, las simetrías y regularidades nos brindan intuición física y simplicidad matemática. Estas regularidades no se encuentran presentes en vidrios, lo que nos inhabilita a aplicar gran parte de los recursos desarrollados por la materia condensada ordenada. Esta repentina obsolencia de entendimiento, sera el punto de partida de nuestro tratamiento de los vidrios.

\subsubsection{Rigidez de un Vidrio}

Cuando pensamos en las partículas que constituyen un sistema, podemos tomar la temperatura como una medida de la energía cinética del sistema [Stein and Newman, 2013]. A altas temperaturas, la entropía es alta y tenemos un sistema en estado líquido o gaseoso. A temperaturas bajas, las fuerzas atractivas prevalecen, la entropía es mas baja y el sistema asume un estado ordenado y de baja energía, el sólido cristalino. Los líquidos y los cristales, son dos fases diferentes de la materia y la transición entre ellas, es una transición de fase.

Una de las cualidades naturales que esperamos del estado cristalino, es un cierto grado de rigidez generalizada, es decir, cuando uno empuja un átomo en un extremo del cristal, la fuerza se propaga de tal manera a través de dicho cristal, tal que el sólido completo se mueve como una entidad. Hasta aquí no hay sorpresas.

Pero esta condición de rigidez generalizada no debe ser tomada por obvia. Las fuerzas interatomicas son de muy corto alcance $\left(10^{-8} \mathrm{~cm}\right)$, pero ese empujoncito en el extremo del cristal se transmite de manera uniforme en un rango miles de millones de veces mas grande que el alcance de las fuerzas interatómicas. Nuevamente, esto no parece algo muy novedoso. Los átomos se encuentran en un estado de baja energía (cristal) y deformar/romper/torcer este estado, requeriría una gran cantidad de energía.

Sin embargo, debemos notar que la rigidez no es un efecto exclusivo de los cristales y que puede ser tratada como un comportamiento emergente de un sistema con muchas partes interactuantes. Las fuerzas interatómicas son de corto alcance, sin embargo, el fenómeno de rigidez es de largo alcance (largo alcance comparado con las fuerzas) y 
posee una simetría rota.

Por otro lado, un vidrio, es un líquido que se vuelve más y más viscoso y lento a medida que se enfría, hasta que deja de moverse en escalas apreciables por humanos. El problema aquí es que, hasta donde entendemos, no existe una transición de fase entre líquido y vidrio. Tampoco hay una simetría rota o la aparición de un cierto orden a largo alcance. Pero sí hay rigidez.

Comenzamos a ver las dificultades. El vidrio es un tipo de sistema desordenado y gran parte de las herramientas utilizadas en sistemas de materia condensada, se aplica a sistemas ordenadas con simetrías claras que nos permiten el uso de simplificaciones matemáticas, entre otros beneficios.

\subsection{Fenomenología Vítrea}

Antes de pasar a las teorías (sección 2.6) y los modelos que estudiaremos (sección 2.7), primero introduciremos las características salientes de los líquidos sobreenfriados y el comportamiento vítreo, sin detenernos a intentar explicar por qué suceden, sino simplemente cuales son dichos rasgos. Si bien discutiremos fenomenología en términos de comportamientos que surgen al disminuir $\mathrm{T}$, este análisis funciona para otros parámetros de control, como el aumento de la densidad $\rho^{1}$.

Cuando enfriamos un líquido bien por debajo de su $\mathrm{T}_{\mathrm{f}}$, el tiempo de relajación así como su viscosidad crece exponencialmente. Para la viscosidad puede crecer hasta 14 décadas [Cavagna, 2009] en un pequeño rango de T. Este crecimiento poco tiene que ver con lo observado en $T>T_{f}$, donde los cambios son suaves y mucho más moderados. Una cualidad que surge es que este comportamiento es común a muchos líquidos con estructuras microscópicas muy diferentes.

De seguir enfriando el sistema, encontraremos una temperatura $T_{g}$ para la cual el sistema ya no relaja durante el tiempo experimental disponible. Para $T<T_{g}$ la muestra está fuera de equilibrio en la escala de tiempo de nuestro experimento. Hemos formado un vidrio. De manera que podemos tomar como definición de transición vítrea dinámica (o cinética) vítrea a $\mathrm{T}=\mathrm{T}_{\mathrm{g}}$ donde el tiempo de relajación excede al tiempo experimental:

$$
\tau_{R}\left(T<T_{g}\right)>\tau_{\exp }
$$

\footnotetext{
5

${ }^{1}$ Precisamente sera $\rho$ el parámetro de control que usaremos para estudiar el modelo t154 en la sección
} 


\subsection{1. $\quad$ El Desorden de los Vidrios}

Notamos que el desorden de los vidrios, no es el mismo tipo de desorden que se observa en líquidos o gases. En estos últimos, los átomos están ubicados en posiciones aleatorias, pero conforme más alta es la temperatura, más se mueven. Se trata de un desorden que surge de la agitación térmica. Esto nos permite hacer promedios estadísticos mediante técnicas físico-matemáticas.

Los vidrios, se encuentran enfriados súbitamente o templados (del ingles quench), en un estado desordenado de baja temperatura, donde ninguna de las técnicas disponibles para líquidos o sólidos nos brinda información alguna.

No los estudiaremos en este trabajo, pero cabe mencionar que existen materiales cuyo desorden templado no es estructural, como el que surge de líquidos sobreenfriados formadores de vidrios, sino magnético. Esta clase de materiales son llamados vidrios de spin. Los vidrios de spin exhiben frustración (no todos los espines pueden ser felices simultáneamente) y en una traducción de las palabras de uno de sus padres fundadores: El mundo esta hecho de sistemas con intereses en conflicto, desde teoría de juegos hasta un grupo de gente eligiendo un menú en un restaurante, uno comienza a ver que los vidrios de spin no es un mal modelo para muchos aspectos de la vida. [Anderson, 1989]. Aparte de sus aplicaciones directas a materiales magnéticos, los estudios de vidrios de spin son aplicables a campos como la biología, las finanzas, la neurociencia y las ciencias de la computación, entre otros [Zarinelli, 2012].

Otro enfoque para entender el desorden de los vidrios, en particular los estructurales, puede obtenerse si uno considera como un líquido sobreenfriado puede crear un vidrio

\subsubsection{Líquidos Formadores de Vidrios}

Si un líquido es enfriado por debajo de su temperatura de fusión $T_{f}$, éste puede sufrir una transición de primer orden, rompiendo simetría y transformándose en un cristal. Sin embargo, si enfriamos un líquido por debajo de $T_{f}$ de manera que no pueda nuclear, el cristal no se forma y tenemos un líquido sobreenfriado. Este es un estado metaestable (pues para $\mathrm{T}<\mathrm{T}_{\mathrm{f}}$, el estado fundamental es el cristal) que puede considerarse como un equilibrio local en el espacio de configuraciones. De seguir enfriando este líquido sobreenfriado, observamos un incremento exponencial en las viscosidad, hasta que llegamos a $T_{g}$. Es para esta temperatura que el líquido se sale del equilibrio, pues los tiempos de equilibración superan los tiempos experimentales. Es importante notar que $T_{g}$ es una medida totalmente antropocéntrica definida como la temperatura para la cual el sistema alcanza una viscosidad de $10^{13}$ Poise. Nada en particular sucede a esa 
viscosidad, es solo una convención ${ }^{2}$. Sin embargo, cuando el líquido se sale de equilibrio, se ha transformado en un vidrio. El vidrio es sólido, pero por otro lado, el vidrio tiene las mismas simetrías que un líquido, de aquí se tiene que el vidrio es un sólido amorfo.

Un recurso frecuentemente usado al comienzo de presentaciones de trabajos con vidrios, consiste en mostrar imágenes que exhiben configuraciones de líquidos y vidrios, en las que el orador desafía a la audiencia a determinar cuál es cuál a simple vista. Esta aparente imposibilidad de determinar cuál es cuál, surge por que estáticamente, ambos estados se ven igual. Este desafió ha motivado trabajos, en los que se busca justamente una herramienta para poder hacer esta diferenciación estática [Kurchan and Levine, 2009], mediante el estudio de distancias de correlación puramente geométricas.

\subsubsection{La Teoría de Adam Gibbs}

Una posible interpretación de lo que sucede al llegar a $T_{g}$, es que al llevar el sistema fuera del equilibrio, no le estamos dando suficiente tiempo para explorar el espacio de fase y de esta manera, estamos disminuyendo el numero de grados de libertad efectivos accesibles al sistema. Es decir, el sistema deja (por un tiempo) de ser ergódico, sufriendo una ruptura de ergodicidad débil. Decimos que es una ruptura débil, pues cuando el sistema esta a $T<T_{f}$, puede quedar atrapado en un valle del paisaje de energía [Barrat, 2003] durante un cierto (largo) tiempo, hasta que consigue escapar y recorrer otras partes del espacio de fase.

Una posible cuantificación de esta disminución de ergodicidad, puede hacerse utilizando argumentos entrópicos mediante la teoría de Adam-Gibbs. Segun la teoría de Adam-Gibbs [Masiewicz et al., 2015], existe una relación entre el tiempo de relajación $\tau_{R}$ y el exceso de entropía $\Delta S_{\text {ex }}(T)=S(T)-S_{\text {cristal }}$, donde $S_{\text {cristal denota la entropía }}$ del cristal perfecto.

Podemos relacionarla con la formula de Arrhenius, en la expresión [Pauling, 2014]:

$$
\tau_{R}=\tau_{0} \exp \left(\frac{B}{T \Delta S_{e x}(T)}\right)
$$

Donde B contiene constantes. Esta poderosa expresión vincula la entropía con el tiempo de relajación, pero si no conocemos la forma de $S(T)$ no podemos avanzar demasiado. Recién cuando consideremos la crisis de entropía de Kauzmann podremos obtener un mejor entendimiento.

\footnotetext{
${ }^{2}$ Otra convención para definir $\mathrm{T}_{\mathrm{g}}$ es cuando el tiempo de relajación de la muestra excede el tiempo experimental de $10^{3}$ segundos
} 


\subsubsection{Crisis de Entropía y Tiempo de Relajación}

Si bien no es posible realizar medidas de equilibrio para $T \leqslant T_{g}$, podemos intentar interpretar y extrapolar cantidades medibles para extraer intuiciones. Esto fue lo que hizo Kauzmann [Debenedetti and Stillinger, 2001], cuando realizo extrapolaciones sobre el exceso de entropía $\Delta S_{\text {ex }}(T)$, encontrando que para algunos materiales, había una temperatura $T_{K}>0$ para la cual, la entropía del vidrio igualaba a la del cristal. Más aún, existían valores de temperatura $\mathrm{T}>0$ para los cuales el líquido tenia una entropía menor a la del cristal el cual es el estado mínimo de energía. Este hallazgo fue conocido como la crisis de entropía y fue el propio Kauzmann, quien postulo que la extrapolación vale hasta $T=T_{K}$, mientras que hay teorías [Debenedetti and Stillinger, 2001] que proponen la existencia de una transición de fase termodinámica al misterioso vidrio ideal en $T=T_{K}$, mostrando que $T_{g}$ es simplemente una precursora a una transición de fase termodinámica que no se ha observado aún y la fenomenología vítrea observada, es evidencia de la aproximación a tal transición ideal.

Si utilizamos que $\Delta S_{\text {ex }}\left(T_{K}\right)=0$, podemos desarrollar:

$$
\Delta S_{e x}(T) \simeq A\left(T-T_{K}\right)
$$

De manera que si reemplazamos en la ecuación 2.3 y agrupamos las constantes A y $B$ en $B^{\prime}$, resulta:

$$
\tau_{R}=\tau_{0} \exp \left(\frac{B^{\prime}}{T-T_{K}}\right)
$$

Obteniendo una expresión que depende de $T_{K}$. Usando valores medidos de $T$ y $\tau_{R}$ en la región metaestable, podemos extrapolar un valor de $T_{K}$ mediante el uso del ajuste empírico de la ley Vogel-Fuchter-Tamman (VFT). En su trabajo [McKenna, 2008], McKenna estudia una variedad de datos experimentales de formadores de vidrios, mostrando que todos presentan una relación entre T y $\tau$ dada por VFT. Si realizamos un gráfico tipo Angell [Debenedetti and Stillinger, 2001], podemos ver que del crecimiento explosivo del tiempo de relajación (o la viscosidad) en la región sobreenfriada, surgen dos clasificaciones. Formadores de vidrios cuyas $T_{K} \simeq 0$, a los que llamaremos fuertes, y otros cuya $T_{K}>0$, a los que llamaremos frágiles.

En la sección 2.4.3 veremos argumentos dinámicos (espinodal cinética), también debidos a Kauzmann, por los cuales la crisis de entropía podría ser evitada, quedando prohibida la transición vítrea ideal. 


\subsubsection{Difusión y la Relación de Stokes-Einstein}

Consideremos la ecuación de difusión unidimensional para la concentración $\rho(x, t)$ [Cavagna, 2009] :

$$
\dot{\rho}(x, t)=D \frac{\partial \rho}{\partial x^{2}}
$$

Donde D representa el coeficiente de difusión. Podemos vincular estas cantidades con

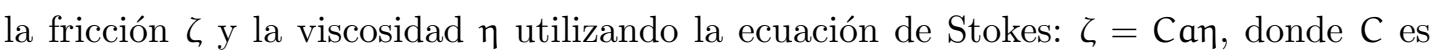
una constante dependiente de las condiciones de borde y a es el radio de una esfera moviendose en un líquido de viscosidad $\eta$. Mediante una serie de pasos [Cavagna, 2009], es posible vincular estas cantidades en la relación de Stokes-Einstein:

$$
\mathrm{D \eta}=\frac{\mathrm{k}_{\mathrm{B}} \mathrm{T}}{\mathrm{Ca}}
$$

Esta relación vincula el coeficiente de difusión con la viscosidad y si bien, solo es válida para la difusión de una esfera mucho mayor al tamaño de las moléculas la del fluido, resulta sorpresivamente precisa en la descripción del coeficiente de auto-difusión D en casos donde la partícula y el fluido son de tamaños similares.

Sin embargo, se observa que para líquidos sobreenfriados [Cavagna, 2009, Darst et al., 2010],

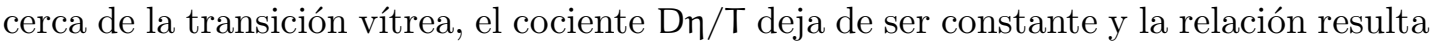
violada.

\subsubsection{Relajación en Dos Pasos}

Hemos visto que para muestras sobreenfriadas que se aproximan a $T_{g}$, el tiempo de relajación $\tau_{R}$ crece exponencialmente. De manera que la correlación dinámica, a diferencia de una correlación estática, sída cuenta de que hay un cambio significativo de comportamiento al aproximarnos a $T_{g}$, pero más aún, aparte de este efecto cuantitativo $\left(\tau_{\mathrm{R}}\right.$ crece fuertemente) surge un cambio de comportamiento cualitativo:

Como muestra la figura 2.1, al llevar al sistema a la región sobreenfriada, la relajación se desdobla. El sistema relaja hasta un valor intermedio, en el cual permanece un tiempo variable (meseta), luego del cual continua relajando hasta 0 [Cavagna, 2009]. Denotaremos a la primer relajación rápida $\beta$ y a la relajación posterior a la meseta, relajación lenta $\alpha$. Tomaremos este cambio en la forma de la curva de relajación, en particular la formación de una meseta, como una de las marcas distintivas de aproximarnos a la transición vítrea. Lógicamente, localizar el valor de T para el cual se comienza a formar la meseta, resulta impreciso, pero lo que podemos garantizar es que para cuando la meseta se observa, estamos en presencia de fenomenología propia de 


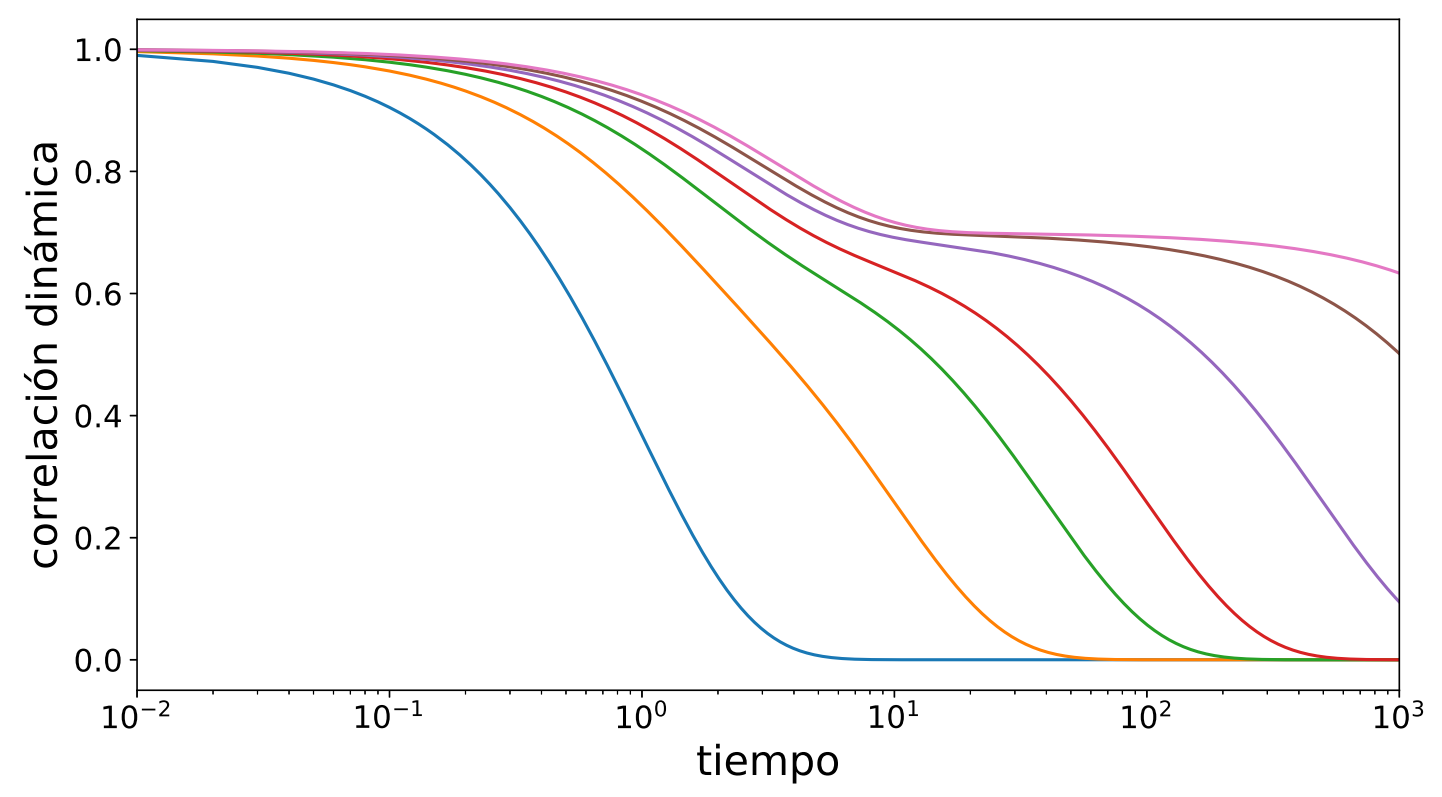

Figura 2.1: Caricatura de la relajación en dos pasos, inspirada en la correlación dinámica para un sistema con interacción Lennard Jones [Cavagna, 2009]. Observamos un decaimiento rápido (relajación $\beta$ ), seguido de una meseta que se hace más y más sostenida en el tiempo al enfriar el sistema. Finalmente el sistema experimenta un decaimiento lento (relajación $\alpha$ ).

un vidrio. La meseta nos da más información. El crecimiento gradual de la meseta nos indica, es que la transición entre relajación exponencial a relajación en dos pasos, es continua.

La relajación en dos pasos, sugiere que usar un único tiempo (o una única escala temporal) ya no es una buena descripción del sistema. La duración de la relajación $\beta$ no depende fuertemente de $T$ mientras que el tamaño de la meseta y la consiguiente relajación $\alpha$ resultan sumamente sensibles. Es decir, la separación de escalas temporales se acrecienta a medida que $T$ se aproxima a $T_{g}$. Daremos una interpretación de este comportamiento en la sección 2.3 .

\subsubsection{La Jaula}

A continuación analizaremos en detalle la meseta producida en la relajación en dos pasos (ver sección 2.3.6), mediante un observable que analice el movimiento de las partículas. Para ello, recurrimos al desplazamiento cuadrático medio (DCM) que se define como:

$$
\left\langle r^{2}(t)\right\rangle=\frac{1}{N} \sum_{i}\left\langle\left\|\overrightarrow{x_{i}}(t)-\overrightarrow{x_{i}}(0)\right\|^{2}\right\rangle
$$


Donde $\overrightarrow{x_{\mathfrak{i}}}(\mathrm{t})$ representa la posición en función del tiempo $t$ de la i-esima de un total de $\mathrm{N}$ partículas. Del comportamiento de líquidos esperamos que el DCM tenga un comportamiento balístico $\left\langle\mathrm{r}^{2}(\mathrm{t})\right\rangle \mathrm{t}$ para tiempos cortos, luego pasando a partículas en un régimen difusivo donde $\left\langle\mathrm{r}^{2}(\mathrm{t})\right\rangle \mathrm{t}^{2}$, debido a colisiones.

Sin embargo, para el régimen sobreenfriado aproximándose a $T_{g}$, se produce la aparición de una meseta entre los comportamientos t y $t^{2}$ [Cavagna, 2009]. En este caso, la meseta tiene una interpretación directa. Las partículas apenas vibran alrededor de posiciones fijas. Durante este periodo, las partículas resultan atrapadas en una cierta pequeña región, fenómeno denominado enjaulamiento. Notamos que no se trata de la aparición de partículas o fuerzas nuevas actuando, son las mismas partículas las que generan esta jaula que las confina. En términos de la relajación en dos pasos (ver figura 2.1), podemos decir que cualitativamente en la relajación $\beta$ las partículas se mueven dentro de la jaula y recien durante la relajación $\alpha$ efectivamente escapan fuera de la jaula y se alejan de sus posiciones en $t=0$.

Esta no es una explicación del comportamiento, sino una interpretación. Notamos también que este comportamiento en el DCM, no se trata de una interpretación de un comportamiento en el espacio de fase, sino de una observación directa del espacio real, donde las partículas se mueven.

\subsubsection{Caída de $c_{p}$}

Varias cosas apreciables experimentalmente suceden en torno a $T_{g}$. Al estar fuera de equilibrio, el sistema ya no tiene tiempo suficiente de explorar el espacio de fase, lo que implica una fuerte pérdida de grados de libertad accesibles. Una consecuencia de esto es una fuerte caída en el calor especifico $c_{p}$ para $T=T_{g}$ [Cavagna, 2009]. Esto explica del hecho que $c_{p}\left(T<T_{g}\right)$ resulta ligeramente superior al $c_{p}$ (cristal). En un cristal, el movimiento de las partículas se debe a vibraciones de las mismas alrededor de sus posiciones de equilibrio ordenadas. No hay reacomodamientos. La repentina similitud entre calores específicos nos indica que para el vidrio, las partículas se encuentran vibrando alrededor de sus posiciones de equilibrio desordenadas, casi sin reacomodamientos. En el vidrio la ergodicidad esta dinámicamente rota, en la misma manera que para el cristal esta termodinámicamente rota, confinando las partículas a un mínimo local (el mínimo absoluto es el cristal) en el espacio de fase. El resultado puede parecer el mismo, tanto el vidrio como el cristal, están atrapados en una configuración en la que el sistema no recorre todo el espacio de fase.

Esto muestra una diferencia central entre la ruptura dinámica de ergodicidad para un vidrio (mínimo relativo en espacio de fase), y la ruptura termodinámica de ergodici- 
dad para el cristal (mínimo absoluto en espacio de fase). Es mas, el calor especifico $c_{p}$ es una cantidad definida en la termodinámica del equilibrio. Si esperásemos un tiempo muy largo (millones de años quizás), le daríamos tiempo al sistema para recuperar ergodicidad y recorrer todo el espacio de fase, dándonos un valor de $c_{p}$ similar al del líquido. El sólido por otra parte, está atrapado por siempre en ese mínimo y la ergodicidad está eternamente rota. En este aspecto, comparar vidrios con cristales, es comparar tiempos extremadamente largos con tiempos infinitos.

\subsubsection{La Exponencial Estirada}

La evolución de un sistema próximo a $T_{g}$ resulta heterogénea, lo que nos lleva a pensar que sus relajaciones no se producen de manera global, sino por regiones, cada una con un tiempo $\tau$ [Lindsey and Patterson, 1980], de manera que el efecto global no es una relajación exponencial, sino una curva exponencial estirada propuesta por Kohlrausch-Williams-Watts (KWW):

$$
C(t)=C_{0} \exp \left(-(t / \tau)^{\beta}\right)
$$

Notar que el coeficiente $\beta<1$ no está relacionada a la rápida relajación $\beta$ sino con la relajación $\alpha$. El valor de $\beta$ representa un exponente que decrece con el aumento de la temperatura, así distinguiendo el comportamiento en la zona cercana a $T_{g}$ del comportamiento exponencial en $\mathrm{T}>\mathrm{T}_{\mathrm{g}}$.

Existen argumentos [Cavagna, 2009] que proponen, que el fenómeno de relajación en la proximidad a la zona vítrea no es una colección de distintas relajaciones homogéneas, que al promediarse se vuelven exponenciales estiradas, sino que la no-exponencialidad es un fenómeno intrínseco inclusive a nivel local, que surge debido al entorno desordenado que cada partícula ve a su alrededor.

En el trabajo Heitjans y colaboradores [Heitjans and Kärger, 2006] $\beta$ puede tomar valores experimentales entre 0.3 y 0.7 (dependiendo del material). Esta exponencial estirada, se aleja marcadamente del comportamiento $\beta=1$ de la relajación de Debye, la cual esta caracterizada por un proceso activado con una única energía de activación.

Nuestra $C(t)$ decae rápidamente para tiempos cortos con una cola larga, señalando el proceso de transporte en medios desordenados. En sistemas vítreos, esta función obedece un comportamiento de escala de tiempo-temperatura, que satisface que todas las medidas a diferentes temperatura $T$ deben de caer sobre una curva maestra, de hacer $\mathrm{C}(\mathrm{t} / \tau(\mathrm{T}))$. Hablaremos mas sobre esto en la sección 2.3.10. 


\subsubsection{Correlaciones Dinámicas y Envejecimiento}

Además de los argumentos estáticos que ya hemos provisto, quizás la diferencia fundamental entre un vidrio (fuera de equilibrio) y un cristal, viene por el hecho de que si bien cantidades como la energía o volumen parecen casi constantes en el tiempo, cantidades de dos tiempos (como funciones de correlación dinámica) muestran un comportamiento totalmente novedoso. A diferencia de los sólidos, los vidrios exhiben dependencia explicita de dos tiempos (en vez de su diferencia).

Venimos hablando de que la transición vítrea es un fenómeno dinámico. Resulta lógico que estudiemos cantidades dinámicas en busca de un comportamiento que nos permita distinguir una muestra aproximándose a $T_{g}$ de un líquido a alta temperatura. Para esto, podemos considerar el estudio de funciones de correlación dinámicas. Consideremos una función genérica $\varphi$ y su correlación de este tipo:

$$
\mathrm{C}\left(\mathrm{t}_{1}, \mathrm{t}_{2}\right)=\frac{1}{\mathrm{~N}} \sum_{\mathrm{k}=1}^{\mathrm{N}}\left\langle\varphi_{\mathrm{k}}\left(\mathrm{t}_{1}\right) \varphi_{\mathrm{k}}\left(\mathrm{t}_{2}\right)\right\rangle
$$

Notemos que tenemos que una cantidad $\varphi_{k}(t)$ relativa a la partícula $k$ en el tiempo $t$. $\mathrm{Si}$ el sistema esta en equilibrio, existe invariancia translacional en el tiempo y haciendo $t=t_{2}-t_{1}$, esta función pasa a depender de solo 1 variable:

$$
C(t)=\frac{1}{N} \sum_{k=1}^{N}\left\langle\varphi_{k}(t) \varphi_{k}(0)\right\rangle
$$

Podemos pensar que existe un tiempo de relajación $\tau_{\varphi}$ asociado a la cantidad $\varphi$, pero en altas temperaturas $\mathrm{T}$ existe una única escala temporal intrínseca, de manera que los distintos observables (y sus $\tau$ asociados) serán re-escaleos unos de otros. $\mathrm{C}(\mathrm{t})$ es una medida de la memoria del sistema, es decir, de como las correlaciones dentro del sistema decaen en el tiempo. Podemos esperar que un sistema a una temperatura por encima de la temperatura de fusión, $T>T_{f}$, decaiga principalmente mediante un régimen disipativo, típicamente descripto por:

$$
C(t)=C_{0} \exp (-t / \tau)
$$

Otra consecuencia de la no invariancia translacional en el tiempo que surge al aproximarnos a $T_{g}$ es que, como los humanos, los vidrios sufren envejecimiento.

Una forma en que el envejecimiento se manifiesta, es que las curvas de correlación $C(t)$ cambian, a medida que transcurre el tiempo. Es decir, si aguardamos un tiempo $t_{w}$ en hacer una medida, las curvas $\mathrm{C}\left(\mathrm{t}, \mathrm{t}_{w}=0\right)$ y $\mathrm{C}\left(\mathrm{t}, \mathrm{t}_{w} \neq 0\right)$ brindan resultados distintos, 
manifestandose como una relajación $\alpha$ con duración mayor. Esto es una característica típica de estar fuera de equilibrio, la invariancia traslacional en el tiempo esta rota, dando $\mathrm{C}\left(\mathrm{t}, \mathrm{t}_{w}\right) \neq \mathrm{C}\left(\mathrm{t}-\mathrm{t}_{w}\right)$. Para sistemas que exhiben este fenómeno, podemos estudiar el escaleo de la relajación $\alpha$, mediante un exponente $v$ dado para $t_{w}>>1$ :

$$
C\left(t, t_{w}\right)=f\left(t / t_{w}^{v}\right)
$$

De manera que para sistemas en equilibrio $t$ es suficiente, mientras que para fuera del equilibrio, en presencia de envejecimiento, debemos considerar dos tiempos $t_{1}, t_{2}$.

\subsection{Cristalización}

Hasta ahora, hemos discutido fenómenos pertinentes a los sistemas vítreos. Pero quizás primero debamos preguntarnos, que le pasa a un sistema que cuando $T<T_{\text {f }}$. Es decir, por que es que no todos los sistemas automáticamente cristalizan ni bien su temperatura cae por debajo de su punto de fusión. Además de argumentos termodinámicos que consideran fases de equilibrio sólidas o líquidas, debemos hacer consideraciones cinéticas, relacionadas a metaestabilidad y barreras de energía.

Sabemos que el líquido metaestable posee una energía y una entropía mayor a la del cristal para esa misma temperatura. El balance entre energía, entropía y como evoluciona el sistema, esta regulado por la temperatura $T$ mediante la expresión de la energía libre $\mathrm{G}=\mathrm{U}-\mathrm{TS}$. Para $\mathrm{T}>\mathrm{T}_{\mathrm{f}}$ el balance de energía y entropía, resulta en una energía libre menor, cuando la entropía es mayor, resultando en que el líquido es la fase estable. Análogamente, la fase estable para $\mathrm{T}<\mathrm{T}_{\mathrm{f}}$ sera la del cristal. Sin embargo, la cristalización no necesariamente es un proceso automático cuando $T<T_{f}$. Si bien la formación de un núcleo cristalino es favorecida por una $T<T_{f}$, la formación de una interfase entre cristal y líquido, resulta en un precio energético adicional debido a efectos de superficie.

Es por esto, que primero debemos orientar nuestros esfuerzos a entender que es la cristalización y los dos eventos que la caracterizan: Nucleación y Crecimiento Cristalino.

\subsubsection{Nucleación}

El cambio total en energía libre, debida a la formación de un núcleo de cristal en d dimensiones de radio R, viene dado por el cambio de energía libre de Gibbs G:

$$
\Delta \mathrm{G}(\mathrm{R})=\sigma \mathrm{R}^{\mathrm{d}-1}-\delta g \mathrm{R}^{\mathrm{d}}
$$


Donde $\sigma$ representa la tensión superficial, asociada a la energía libre por unidad de área en formar la interfaz entre fases líquido-sólido, $\delta \mathrm{g}$ representa la diferencia de densidad de energía libre entre la fase líquida y la sólida, dada por:

$$
\delta g=g_{\text {líq }-g_{\text {sól }}}(2.15)
$$

Por definición, $\delta g\left(T=T_{f}\right)=0$, siendo creciente para $T<T_{f}$. En general, puede ajustarse en función de la entalpía molar de fusión $\delta$ h y el volumen molar de un cristal $v$, con $\mathrm{T}<\mathrm{T}_{\mathrm{f}}$, usando [Cavagna, 2009]:

$$
\delta g(T)=\frac{\delta h}{v}\left(1-\frac{T}{T_{f}}\right)
$$

Donde $\delta$ h es la entalpía molar de fusión y v es el volumen molar del cristal. En esta expresión y en la ecuación 2.14, queda expuesta la competencia entre costo de superficie $\sigma$, que se opone a formar un núcleo, y el empuje termodinámico $\delta g$ que lo favorece.

Maximizando 2.14 para $T \neq T_{f}$, hallamos un valor critico $R=R_{C}$ que nos brinda el valor extremo:

$$
\Delta \mathrm{G}\left(\mathrm{R}=\mathrm{R}_{\mathrm{C}}\right)=\frac{\sigma^{\mathrm{d}}}{\delta \mathrm{g}^{\mathrm{d}-1}}
$$

Esta expresión cuantifica la barrera de energía libre para formar un núcleo estable.

Adicionalmente, podemos ver de 2.14, que para $\mathrm{T}=\mathrm{T}_{\mathrm{f}}$ formar un núcleo cristalino resulta una desventaja en términos de superficie. Este es el motivo por el cual, para producir la transición de fase, es necesario un sobreenfriamiento de la muestra. También vemos que a medida que $\mathrm{T}$ disminuye, $\delta \mathrm{g}$ aumenta, disminuyendo la barrera energética y favoreciendo la nucleación.

\subsubsection{Dinámica de la Nucleación}

Conocer los tiempos involucrados relacionados a la cristalización es central para comprender como es posible que se forme un vidrio. Hasta ahora hemos tenido consideraciones independientes del tiempo que nos indican si un sistema minimiza su energía manteniéndose en una fase o en la otra.

Ahora consideraremos los tiempos involucrados en la formación de un núcleo de cristal de tamaño $R_{C}$. Utilizando la expresión de Arrhenius para procesos activados [Debenedetti and Stillinger, 2001] y usando la barrera de energía hallada en la ec. 2.17, tenemos el tiempo de nucleación $\tau_{N}$ como:

$$
\tau_{\mathrm{N}}=\tau_{0} \exp \left(\frac{\Delta G\left(R_{C}\right)}{k_{B} T}\right)=\tau_{0} \exp \left(\frac{\sigma^{d}}{k_{B} T \delta g^{d-1}}\right)
$$


Ignorando $\tau_{0}$ momentáneamente y prestando atención al argumento de la exponencial, notamos que no hay referencia al volumen en esta fórmula. Esta observación es notable, pues la formación de núcleos es un proceso estocástico y local. Es decir, en cada región del sistema, hay una chance de formar un núcleo cristalino. Si incrementamos el tamaño del sistema, habrá más regiones y la chance de formar un cristal en alguna de todas las regiones aumenta.

Esta situación se aclara, si miramos una cantidad más fundamental de la nucleación: la tasa de nucleación $\mathfrak{j}_{\mathrm{N}}$, que nos indica la cantidad de núcleos formados por tiempo y por unidad de volumen [Cavagna, 2009]. Si el volumen $V$ es grande, $j_{N}$ resulta una constante dada por:

$$
j_{N}=j_{0} \exp \left(-\frac{\sigma^{d}}{k_{B} T \delta g^{d-1}}\right)
$$

Ahora, si la tasa por unidad de volumen para formar un núcleo cristalino es constante, entonces el tiempo necesario para formar un cristal debe escalear como $\mathrm{V}^{-1}$, de donde sale:

$$
\tau_{\mathrm{N}}=\frac{1}{j_{\mathrm{N}} V}
$$

De esta manera aclarando la dependencia de $\mathrm{V}$ con la ecuación 2.19: $\tau_{0}$ ha de contener un termino $1 / \mathrm{V}$.

Esto nos dice que en muestras de volumen grande, la nucleación puede producirse antes, dejando rápidamente la fase metaestable. De manera que una buena estrategia experimental para observar meta-estabilidad, es trabajar con muestras pequeñas.

Otra observación que podemos hacer, es que $\mathrm{V}$ permanece fuera de la exponencial de $\tau_{N}$. Esto nos indica que cambios sutiles en $V$ no producen consecuencias drásticas, mientras que un cambio en $T$, produce profundos cambios en $\tau_{N}$. Además, vemos de las ecuaciones 2.16 y 2.18 , que $\tau_{\mathrm{N}}$ diverge para $\mathrm{T}=0$ y $\mathrm{T}=\mathrm{T}_{\mathrm{f}}{ }^{3}$. Analizando este comportamiento, vemos que $\tau_{N}$ debe tener un mínimo $\tau_{\text {min }}$ entre esos puntos, donde la cristalización se vuelve más probable.

\subsubsection{La Temperatura Espinodal Cinética}

Si consideramos que existe un $\tau_{\min }$, entonces una posible manera de evitar una cristalización, seria enfriar el sistema mas rápidamente que un ritmo de enfriamiento $r$, que segun $\tau_{\min }$ va como $r_{\min }=1 / \tau_{\min }$. Ahora, recordemos que según la expresión 2.5, mientras mas baja $\mathrm{T}$ mayor es el tiempo de relajación, es decir, el tiempo que debemos esperar para que el sistema relaje. Esto nos plantea una contraposición si queremos

\footnotetext{
${ }^{3}$ Este comportamiento vale si asumimos que $\tau_{0}$ es independiente de T. Esto no siempre es cierto, pero no le quita toda la validez al análisis
} 

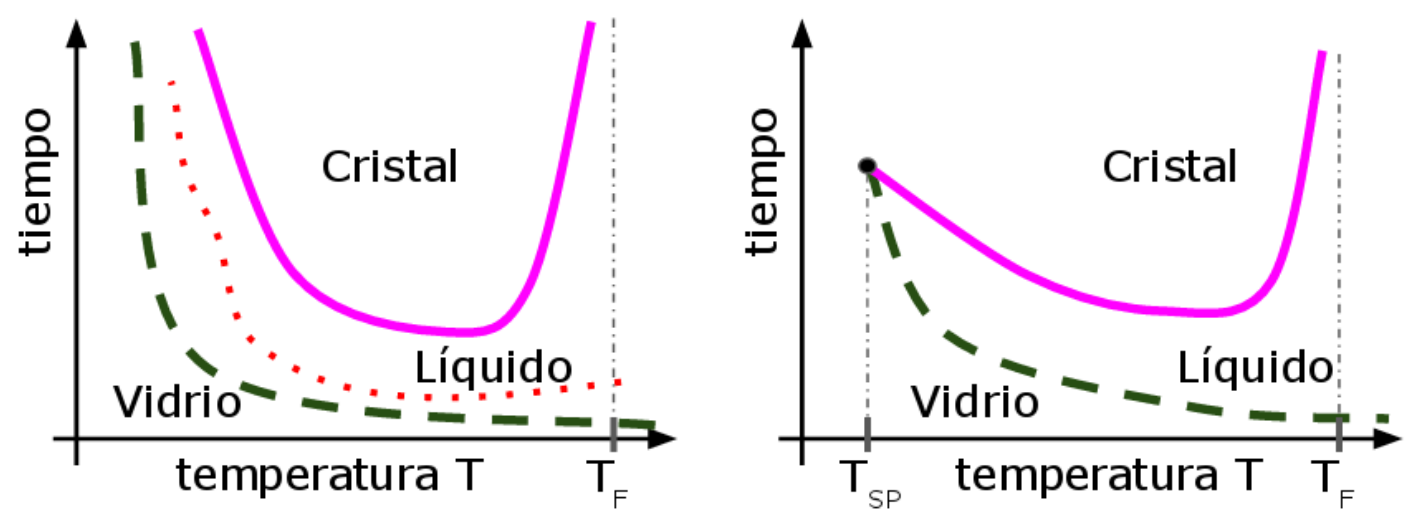

Figura 2.2: A la izquierda: Este esquema muestra la importancia en la velocidad de los procesos de enfriamiento por debajo del punto de fusión $T_{F}$. Un enfriamiento veloz, lleva al sistema a un estado vitreo. Un enfriamiento muy lento, lleva el sistema a cristalizar. La linea punteada, representa una posible ruta de enfriamiento $r(T)$. A la derecha: Este esquema es similar al anterior pero considera el escenario de la ecuación 2.21. No hay ruta de enfriamiento que permita evitar la cristalización o vitrificación por debajo de TSP.

evitar la cristalización y mantenernos en la región de líquido metaestable:

Debemos enfriar suficientemente rápido, como para no darle tiempo al sistema para que comience la nucleación, pero no tan rápido, como para sacarlo fuera de equilibrio. Podemos entonces pensar, que la manera de lograr un líquido sobreenfriado de baja temperatura, es realizar un enfriamiento no lineal, una ruta térmica $r(T)$. Esta ruta de enfriamiento, puede plantearse como se muestra en la figura 2.2.

Sin embargo, existe la posibilidad de que para ciertos valores de $T$ en algunos sistemas, $\tau_{R}>\tau_{N}$, resultando imposible mantener el líquido metaestable. La temperatura bajo la cual sucede esto, se conoce como temperatura espinodal cinética $T_{S P}$ y viene dada por:

$$
\tau_{N}\left(T_{S P}\right)=\tau_{R}\left(T_{S P}\right)
$$

Queda claro, que si existe una espinodal cinética, resulta imposible hacer medidas de equilibrio sóbre el líquido sobreenfriado para $T<\mathrm{T}_{\mathrm{SP}}$, pues la nucleación comienza antes de que pueda equilibrarse la fase líquida.

Notamos que esta es una cantidad cinética, pues depende de cantidades dinámicas. Aclaramos que no depende del protocolo experimental, sino que es una propiedad del material.

La existencia de $T_{S P}$ es el argumento propuesto por Kauzmann, que evita la paradoja descrita en la sección 2.3.4, dado que si $\mathrm{T}_{\mathrm{SP}}>\mathrm{T}_{\mathrm{K}}$, entonces el sistema cristaliza antes de alcanzar $\mathrm{T}_{K}$ y no se produce la crisis de entropía. 


\subsubsection{Formación de Cristales}

En la sección 2.4.2 consideramos a $\tau_{\mathrm{N}}$ y su dependencia con $\mathrm{V}^{-1}$. Podríamos preguntarnos, por que no simplemente aumentar el tamaño del sistema hasta disminuir $\tau_{\mathrm{N}}$ tanto como querramos. La razón por la que este argumento no funciona, es que si bien la nucleación es un fenómeno gobernado tensión superficial y energía libre, el crecimiento del cristal esta gobernado por la viscosidad del líquido y por como las partes de este se difunden.

Debemos recordar que la nucleación es un fenómeno local, de manera que aunque se forme un núcleo cristal en una posición $X$, para que el cristal crezca hasta una posición $Y$ a una cierta distancia, dicho núcleo debe crecer desde $X$ hasta $Y$. Este crecimiento puede retardarse, e incluso bloquearse, por falta de encaje entre dominios cristalinos que crecieron independientemente y que al entrar en contacto, no pueden compatibilizar sus geometrías. Sin embargo, hay que notar que una vez que se produce nucleación, el sistema ya no es mas un líquido, sino un cristal.

La cristalización es la combinación de nucleación y crecimiento de cristales. Podemos destacar que un régimen de cristales pequeños es indetectable, pero se encuentra fuera de la fase líquida. Esto nos indica que con tiempo suficiente, el sistema cristalizara. No debemos confundir esta situación de evolución lenta como la de un sistema fuera de equilibrio, como lo es un vidrio.

\subsubsection{La Función de Correlación de Pares g(r)}

Para poder cuantificar la presencia de orden en las posiciones de las partículas en el tiempo, es preciso que definamos una serie de herramientas matemáticas que nos permitan estudiar correlaciones espaciales.

La función de correlación de pares $\mathrm{g}(\mathrm{r})$ es una medida de la probabilidad de encontrar una partícula a una distancia $r$ medida desde una partícula de referencia dada, relativa a la de un gas ideal. El algoritmo general para el cálculo de g(r) involucra el determinar cuántas partículas se encuentran a una distancia $r+d r$ de una partícula de referencia.

Esta función usualmente se calcula determinando la distancia entre todos los pares de partículas y colocando los datos en un histograma. Entonces, el histograma se normaliza con respecto a un gas ideal completamente decorrelacionado. De esta manera se mide el orden respecto al desorden de un gas ideal.

Para sistemas discretos en 2 dimensiones, usando funciones $\delta$ de Dirac, $g(r)$ resulta 


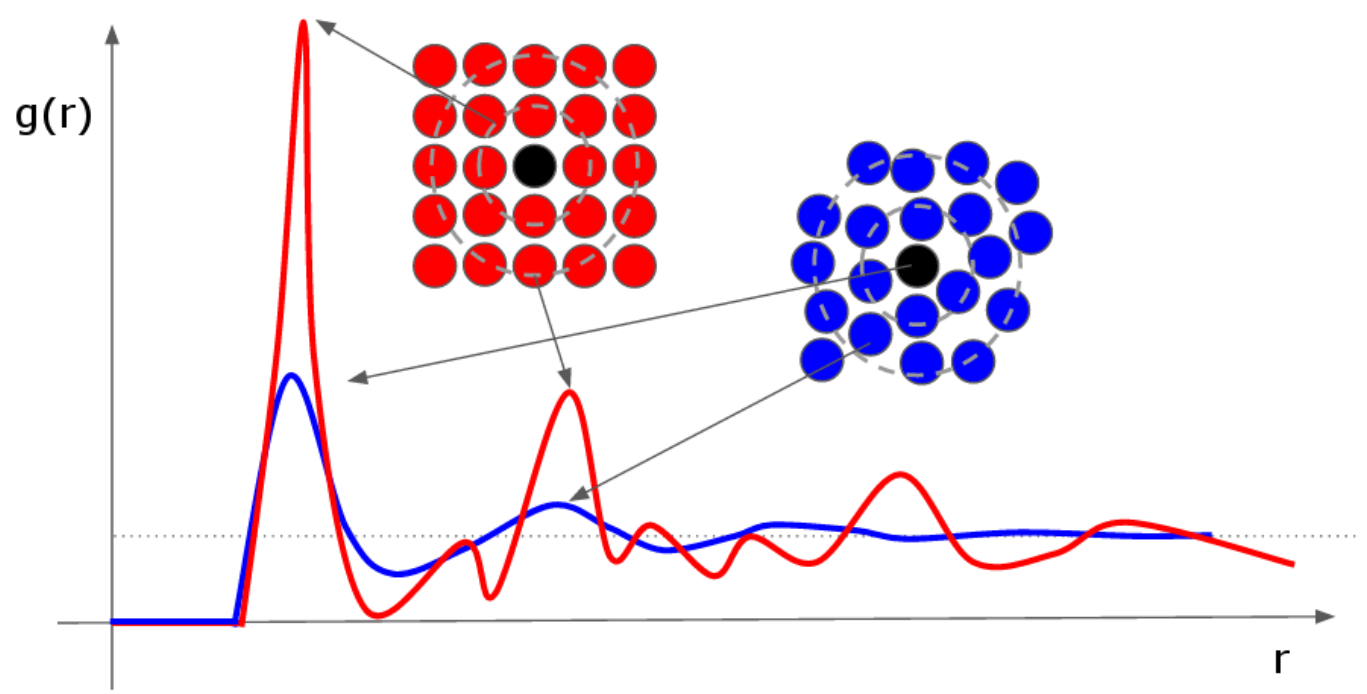

Figura 2.3: Ejemplo cualitativo para g(r), inspirado en [Lu and Szpunar, 1997], para un sistema líquido (azul) y uno cristalino (rojo). La rápida atenuación de la curva del líquido indica la falta de orden de largo alcance.

[Hansen and McDonald, 1990]:

$$
g(r)=\frac{1}{2 \pi r N \rho} \sum_{i=1}^{N} \sum_{j \neq i}^{N}\left\langle\delta\left(r-\left|r_{j}-r_{i}\right|\right)\right\rangle
$$

Algunas propiedades de $g(r)$, a medida que se incrementa $r$ (ver figura 2.3), son:

- Para $r \gtrsim 0, g(r)=0$ indica el tamaño efectivo de los átomos, es decir la distancia mínima a la cual pueden aproximarse entre sí sin interpenetrarse.

- Un primer pico (al incrementar r) es esperable, debido a que este indica el empaquetamiento de átomos próximos.

- Al seguir incrementando $r$, esperamos que un material cristalino, presente más picos de alturas comparables a las del primer pico, mientras que para un líquido o vidrio, la altura de los picos decae a 1 rápidamente, debido al desorden inherente a estos sistemas.

- La rápida atenuación de los picos, indica un decaimiento del orden respecto a la partícula central. Esto puede interpretarse como la ausencia de un orden de largo alcance. 


\subsubsection{El Factor de Estructura Estático $S(k)$}

A diferencia de los cristales, la materia en estado líquido no posee orden de alcance largo, por lo que el factor de estructura no exhibe los picos discretos que caracterizan a la difracción por cristales [Hansen and McDonald, 1990]. La expresión general para $S(\vec{k})$ viene dada por:

$$
S(\vec{k})=\left\langle\frac{1}{N} \sum_{i=1}^{N} \sum_{j=1}^{N} e^{-i \vec{k} \cdot \overrightarrow{r_{i}}} e^{i \vec{k} \cdot \overrightarrow{r_{j}}}\right\rangle
$$

Sin embargo, los líquidos tienen un cierto grado de orden de corto alcance, que depende de su densidad y la magnitud de la interacción entre sus partículas conformantes. Como los líquidos son isotrópicos, el factor de estructura solo depende del módulo del vector $(k=|\vec{k}|)$ y no de su dirección. Si en la expresión general para el factor de estructura se separan los términos diagonales, cuya fase es 0 y son por tanto iguales a la unidad se obtiene:

$$
S(k)=1+\frac{1}{N} \sum_{j \neq k} e^{-i \vec{k}\left(\vec{R}_{j}-\vec{R}_{k}\right)}
$$

$S(k)$, así como $g(r)$, resultan útiles únicamente para verificar que un líquido sobreenfriado presenta (o no) cristalización. Esto se debe, a que el desorden estructural propio de los líquidos o vidrios, no puede ser captado por una función de correlación de dos puntos. Para los experimentales, $\mathrm{g}(\mathrm{r})$ es sumamente atractiva pues mediante técnicas de colisión de neutrones inelasticos [Fischer et al., 2005], es posible medir el factor estático de estructura $S(k)$, el cual esta relacionado a $\mathrm{g}(\mathrm{r})$ mediante:

$$
S(k)=1+4 \pi \rho \int_{0}^{\infty} \mathrm{drr}{ }^{2} \frac{\operatorname{sen}(\mathrm{qr})}{\mathrm{qr}}(\mathrm{g}(\mathrm{r})-1)
$$

Si bien esta conexión tan directa entre experimento y comprensión resulta muy auspiciosa, no lo es para medidas de comportamiento vítreo. Pues muestras muy por arriba o muy por debajo de $T_{f}$ exhiben esencialmente el mismo comportamiento. Esto nos muestra que resulta imposible utilizar el factor de estructura (u otras cantidades estructurales estandar) para determinar si la muestra se encuentra cercana a la transición vítrea.

Sin embargo, estas herramientas confirman que tanto $g(r)$ como $S(k)$, tienen una dependencia muy débil con $\mathrm{T}$ cerca de $\mathrm{T}_{\mathrm{g}}$. Esto resulta confuso, pues es en esa región de temperatura que hay un crecimiento explosivo en los tiempos de relajación. La intuición nos diría que una relajación que aumenta súbitamente, debe tener asociada una distancia de correlación igualmente creciente. La conexión con distancia de correlación 
es debida a que a mayor tamaño de dominios a reacomodar, mas grande debe ser el tiempo de relajación.

En la sección 2.5 vemos que existen funciones de correlación no estándar (puntoconjunto), que presentan una distancia de correlación creciente, confirmando las sospechas de que a mayor reacomodamiento, debe haber un orden de alcance creciente.

\subsection{Correlación Punto Conjunto}

Existe otro tipo de correlación que podemos estudiar, que pertenece al grupo de las correlaciones agnósticas, pues no pretende conocer cual es el parámetro de orden creciente. Nos referimos a la correlación Punto Conjunto (PTS) [Montanari and Semerjian, 2006b]. Para estudiar esta correlación, es preciso partir el sistema en dos partes concéntricas. Una exterior, para la cual las partículas quedan inmovilizadas (o congeladas) y una cavidad interior, para la cual las partículas que pertenecen a dicho volumen pueden evolucionar considerando las restricciones que proveen las partículas en el borde externo a la cavidad $\mathcal{R}$. De esta manera queda definida una superficie, que envuelve a un volumen dentro del cual las partículas interactúan entre sí y con las partículas en dicha superficie. Decimos que las partículas en la cavidad experimentan condiciones de contorno amorfas $(A B C)$.

La correlación Punto Conjunto (PTS) [Cavagna et al., 2012], identifica a ६.PTS (T) como la mínima longitud de escala para la que una superficie formada por partículas congeladas, confinando en su interior partículas que evolucionan con cierta dinámica, deja de tener influencia en el comportamiento de las partículas en el centro del volumen determinado por dicha superficie. Es decir, nos dice hasta que punto los bordes dictaminan el comportamiento del interior de la cavidad $\mathcal{R}$.

Podemos tomar una función de correlación dinámica (ver ec. 2.11), considerando únicamente la correlación en un punto $^{4}$ en el centro de la cavidad concéntrica que describimos. De esta manera, podemos agregar un nuevo parámetro para estudiar, el cual es el tamaño de la cavidad. Si consideramos un sistema en el retículo, podemos llamar a la variable de cada sitio $\sigma$ (considerar el spin por ejemplo). Si definimos una cavidad habrá spines que pertenecen al interior de la cavidad (los llamaremos s) y otros spines que quedan por fuera (los llamaremos $\kappa$ ). Notar que $s=\left\{s_{1}, . ., s_{M}\right\}$ y $\kappa=\left\{\kappa_{M+1}, . ., \kappa_{N}\right\}$. Puesto que usando $A B C$, los espines $\kappa$ permanecen congelados, el estudio que hagamos sobre los spines s, sera influenciado por el arreglo especifico de

\footnotetext{
${ }^{4}$ Por punto nos referimos a un volumen mucho menor al volumen del sistema y menor al de la cavidad.
} 
los spines $\mathrm{k}$ La función de correlación PTS entre un sitio i y la cavidad $\mathcal{R}, \mathrm{C}(i, \mathcal{R})$ [Cavagna et al., 2010] verifica que:

$$
C(i, \mathcal{R})=\left\langle s_{i}\left\langle s_{i}\right\rangle_{\kappa}\right\rangle=\frac{1}{z} \sum_{s, \kappa} e^{-\beta H(s, \kappa)} s_{i} \frac{1}{Z_{\kappa}} \sum_{s^{\prime}, \kappa^{\prime}} e^{-\beta H\left(s^{\prime}, k^{\prime}\right)} s_{i}^{\prime}=\overline{\left\langle s_{i}\right\rangle_{\kappa}^{2}}
$$

Esta cantidad da una medida de autocorrelación para un borde k. Lógicamente, las medidas no pueden depender de un único borde congelado $k$, de manera que en el estudio de PTS, se debe partir de distintas configuraciones del equilibrio, de manera que cuando se determine la cavidad $\mathcal{R}$, el borde que la confina sea distinto cada vez.

Así, podemos partir desde un estado de equilibrio, definir sobre este cavidades de distinto tamaño y estudiar como evoluciona dicha correlación en función del tamaño. Cuando alcancemos un umbral de tamaño para el cual, si seguimos incrementando el tamaño de cavidad, el sistema evoluciona de la misma manera, diremos que los efectos del borde del sistema dejan de ser percibidos en el seno de este. Utilizaremos correlaciones punto conjunto (PTS) para distintas $\mathrm{ABC}$ en la sección 5 con el objetivo de determinar una longitud de correlación $\xi_{\text {PTs }}$. Volveremos sobre el uso de ABC en la prueba de condición inicial BIC (ver sección 3.5.2).

En su trabajo [Biroli et al., 2008], Biroli y colaboradores estudian la pronunciada ralentización de la dinámica de los líquidos sobreenfriados, la cual no exhibe ningún cambio estructural o termodinámico durante el enfriamiento. Observan, que la función de correlación dinámica se vuelve progresivamente no exponencial a medida que la temperatura disminuye, pero no hay rastro de ninguna correlación estática que distinga entre un líquido a alta temperatura y un líquido formador de vidrio sobreenfriado. Sin embargo, la influencia de los bordes se propaga dentro del seno del material. Donde dicha influencia aumenta, con el enfriamiento. Con el incremento de esta longitud de correlación estática, la influencia del borde decae no-exponencialmente.

Otro trabajo relevante, es el de Hocky y colaboradores [Hocky et al., 2012], donde presentan el estudio de la longitud de correlación termodinámica punto-conjunto (ver sección 2.5) para varios modelos canónicos y muestran la conexión cuantitativa entre esta longitud de correlación y los aumentos en tiempos de relajación.

El trabajo [Gradenigo et al., 2013] de Gradenigo y colaboradores, presentan resultados donde las condiciones de contorno amorfas confinan al seno del material, para una geometría plana "sandwich". Sus resultados confirman el uso de correlaciones puntoconjunto como una novedosa herramienta para extraer información de las propiedades estáticas de los líquidos formadores de vidrios y la transición entre fase ergódica a fase no ergódica, en función del tamaño del confinamiento. 


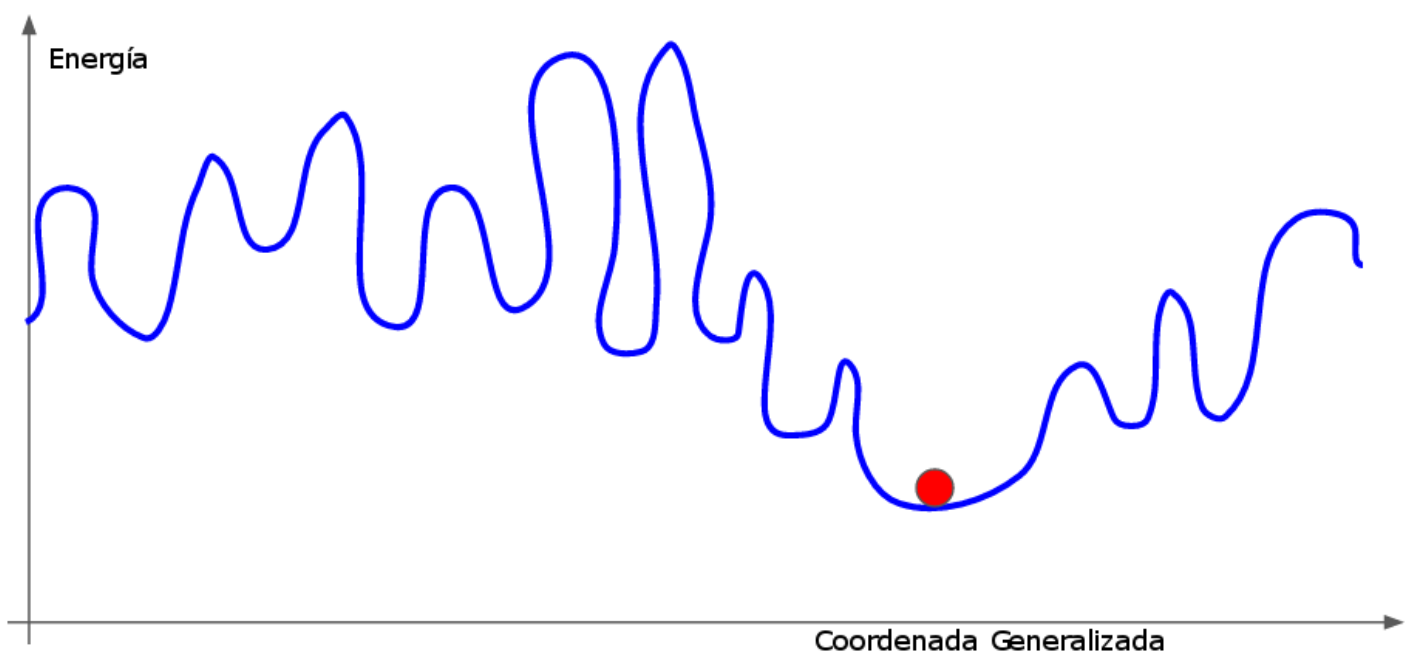

Figura 2.4: Caricatura de un paisaje de energía para un sistema en el continuo que se encuentra en un mínimo absoluto de energía. Existen otros mínimos locales a los que puede acceder, pero hay numerosas barreras a su alrededor.

\subsection{Teorías}

\subsubsection{Topografía: Propuesta de Paisaje de Energía}

Goldstein [Goldstein, 1980] introdujo una propuesta teórica para intentar comprender el comportamiento de los líquidos sobreenfriados. El propuso que los distintos reacomodamientos en el espacio real de las partículas, son simplemente saltos entre distintos valles en un espacio de fase, donde la topología de este paisaje representa la energía del sistema (ver figura 2.4). De esta manera, existe un mínimo absoluto, que corresponde al cristal y muchos mínimos locales, que corresponden a los distintos sólidos amorfos. Así, a menor temperatura, el sistema debe superar barreras más altas de potencial entre valles, lo que hace que el reacomodamiento de partículas en el espacio real se vuelva más infrecuente y visto desde el laboratorio, la viscosidad (o tiempo de relajación) aumente. Así, podemos pensar que el numero de partículas involucradas en un reacomodamiento aumenta, a medida que las barreras entre mínimos locales crecen. De esta manera, un orden amorfo se propaga en el líquido formador de vidrio, cuando la temperatura baja y si en $T=T_{K}$ el exceso de entropía se vuelve 0 , el sistema queda atrapado en uno de dichos mínimos y la ergodicidad queda rota.

\subsubsection{Entonces, ¿Cómo Detectamos un Vidrio?}

En las secciones previas mencionamos la aparición de un orden amorfo, relacionado al aumento del tamaño de las regiones cooperativas que deben reacomodarse al enfriar 
el sistema. Como en otras transiciones de fase, encontrar una función de correlación estática que exhiba una longitud de correlación creciente al acercarnos al punto de transición, nos indicaría que puede existir una transición a un vidrio ideal. El problema, es que aún no es posible encontrar tal longitud de correlación divergente. Como mencionamos antes, distinguir un líquido sobreenfriado alrededor de $T_{g}$ de un líquido sobre $\mathrm{su} \mathrm{T}_{\mathrm{f}}$ todavía resulta imposible usando su estructura estática.

Para sortear este obstáculo, y poder detectar algún tipo de distancia de correlación creciente, buscaremos una distancia de correlación estática, pero usando métodos no estándar en la termodinámica actual. Nos referimos al uso de correlaciones puntoconjunto (ver sección 2.5) en vez del usual punto a punto.

Resulta natural preguntarse, considerando el argumento de paisajes de energía de Goldstein que describimos en 2.6.1, cuales reacomodamientos configuracionales son posibles en el líquido sobreenfriado, dada la gran cantidad de posibles topografías multidimensionales. Y si bien, seria agradable tener una respuesta universal y directa que describa el comportamiento de todos los formadores de vidrios, quizás debamos dejar abierta la posibilidad de que existan distintas descripciones, algunas mas relevantes que otras, que se adecuen a la subfamilia de formadores de vidrio en estudio. O que quizás, las distintas teorías existentes, solo describen bien distintas regiones de la fenomenología.

Del gran abanico de interpretaciones teóricas en el mercado que interpretan la ralentización de la relajación estructural en líquidos sobreenfriados, surgen dos grandes categorías [Stillinger and Debenedetti, 2013]. Una de ellas, puramente enfocada en fenómenos dinámicos y dependientes de tiempo, atribuyendo nada a la termodinámica. La otra, todo lo contrario. En este trabajo, mencionaremos solo dos: Teoría de Modos Acoplados (MCT) y la Teoría Transición Aleatoria de Primer Orden (RFOT).

\subsubsection{Teoría de Modos acoplados MCT}

La teoría de modos acoplados (MCT) [Das, 2004, Gotze and Sjogren, 1992], es un enfoque para estudiar el complejo comportamiento de los líquidos sobreenfriados. Del acoplamiento de funciones de correlación de decaimiento lento, la teoría predice la existencia de una temperatura característica $T_{\mathcal{c}}$ por sobre la temperatura de transición vítrea experimental $\mathrm{T}_{\mathrm{g}}$ para el líquido. $\mathrm{MCT}$, perteneciente a la categoría de dinámica dependiente del tiempo está basada en el formalismo de Mori-Zwanzig y describe la dependencia temporal de las fluctuaciones de densidad a nivel atómico/molecular. Así, genera una predicción para la función de scattering intermedio de un líquido $F(k, t)$, 
con un vector de longitud $k=|\vec{k}|$ :

$$
\begin{array}{r}
F(k, t)=N^{-1}\langle\rho(\vec{k}, 0) \rho(-\vec{k}, t)\rangle \\
\rho(\vec{k}, t)=\sum_{j=1}^{N} \exp \left(i \vec{k} \overrightarrow{r_{j}}\right)
\end{array}
$$

Donde las coordenadas tridimensionales de las $N$ partículas están dadas por $\overrightarrow{r_{1}}, . ., \overrightarrow{r_{N}}$. Si bien en alta temperatura $\left(T>T_{f}\right)$ se presenta el comportamiento esperado de una relajación exponencial para tiempos largos, a medida que la temperatura baja en la región sobreenfriada $\left(T<T_{f}\right)$ aparece una relajación en 2 pasos en forma de una meseta. Sin embargo, para una temperatura $\mathrm{T}_{\mathrm{C}}>0$, predicha por $\mathrm{MCT}$, la meseta no desaparece nunca, quedando el sistema fuera de equilibrio y rompiendo ergodicidad para $T \leqslant T_{C}$. Esto resulta en que las partículas ya no pueden difundirse libremente, sino que quedan localizadas alrededor de sus posiciones iniciales.

Esto resulta problemático, pues mediante tiempos de relajación obtenidos experimentalmente en la región sobreenfriada [Stillinger and Debenedetti, 2013], mediante ajuste de la función con dependencia $\left(T-T_{C}\right)^{\gamma}$, resulta que $T_{C}>T_{g}$. Precisamente esa relación, nos muestra que MCT no contempla los mecanismos de relajación más importantes para el estudio de relajación del líquido sobreenfriado en la cercanía de $T_{\mathbf{g}}$

Esto nos dice que si bien MCT no provee una descripción correcta de la fenomenología vítrea en la cercanía de $T_{g}$, si nos da una descripción aceptable para $T>T_{C}>T_{g}$, incluyendo la existencia de una relajación en dos pasos para la función de scattering intermedio.

\subsubsection{Transición aleatoria de primer orden RFOT (Teoría de Mosaico)}

También conocida como Teoría de Mosaico, la teoría de Transición Aleatoria de Primer Orden (RFOT) se basa en la termodinámica de los líquidos sobreenfriados y la transición vítrea [Stillinger and Debenedetti, 2013], en particular en forma de una transición vítrea ideal, la cual es responsable de la fenomenal ralentización en las relajaciones y consecuente vitrificación.

En su trabajo [Lubchenko and Wolynes, 2007], Lubchenko y colaboradores reveen a RFOT y su capacidad de predecir o explicar fenómenos por sobre y por debajo de $\mathrm{T}_{\mathrm{g}}$. Entre éstos, se presentan las caracteristicas fenomenológicas expuestas en la sección 2.3. El crecimiento explosivo de la viscosidad; el salto en la capacidad calorífica en $\mathrm{T}_{\mathrm{g}}$; la no exponencialidad de relajaciones y su relación con la fragilidad; la heterogeneidad dinámica en líquidos sobreenfriados, atribuyéndola a la estructura de mosaico; 
desviaciones de la ley de VFT; desviaciones de la relación de Stokes-Einstein cerca de $\mathrm{T}_{g}$; envejecimiento y su relación con fragilidad. Los autores encuentran que RFOT tiene gran poder de predicción cuantitativamente en regiones mesoscópicas, de manera de obtener suficiente estadística. Además, para sustancias que presentan comportamiento vítreo junto con tipos de orden adicional, como los polímeros, presentan buen acuerdo cualitativo con los cálculos de RFOT.

Esta teoría incorpora resultados de campo medio para sistemas de spines con interacciones. Los resultados se originan en modelos con desorden templado (del ingles quenched) aleatorio y rango infinito, como el modelo de Sherrington-Kirkpatrick [Sherrington and Kirkpatrick, 1975], modelo de Potts [Wu, 1982] y el modelo de p-spin [Zarinelli, 2012]. La propuesta innovadora de RFOT es la de pensar un vidrio, como una colección aperiódica de mosaicos. Podemos aproximar la energía libre de un mosaico genérico, en condiciones isocoricas, mediante la funcional:

$$
\mathrm{F}^{i n h}[\rho(\overrightarrow{\mathrm{r}})]=\int \mathrm{d} \overrightarrow{\mathrm{r}} \rho(\overrightarrow{\mathrm{r}})[\ln \rho(\overrightarrow{\mathrm{r}})-1]+\frac{1}{2} \iint \mathrm{d} \overrightarrow{\mathrm{r}} \mathrm{d} \overrightarrow{\mathrm{r}^{\prime}}\left[\rho(\overrightarrow{\mathrm{r}})-\rho_{0}\right]\left[\rho\left(\overrightarrow{\mathrm{r}^{\prime}}\right)-\rho_{0}\right] \varphi\left(\overrightarrow{\mathrm{r}}, \overrightarrow{\mathrm{r}^{\prime}}\right)
$$

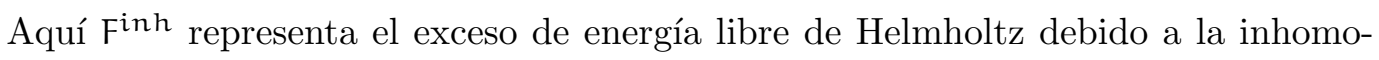
geneidad de la densidad, comparado con la uniformidad de la densidad $\rho_{0}, \rho(\overrightarrow{\mathrm{r}})$ es la densidad no-uniforme y $\varphi\left(\vec{r}, \overrightarrow{r^{\prime}}\right)$ simboliza una energía de interacción adimensional. El primero de los términos en el lado derecho de la ecuación 2.29 representa el costo de energía libre asociado con la localización de las partículas, mientras que el otro termino representa la contribución energética.

Dada la vibración armónica alrededor de sus posiciones de equilibrio, podemos modelar estos movimientos mediante desplazamientos gaussianos y parámetros variacionales $\alpha$ que nos permiten cuantificar la localización. Insertando este modelización de la vibración en la ecuación 2.29 resulta que la energía libre desarrolla mínimos metaestables con respecto a $\alpha$ para temperaturas suficientemente bajas (o densidades $\rho_{0}$ suficientemente altas). Y si bien, estos mínimos corresponden a una transición de congelamiento de primer orden, el congelamiento es en forma de un gran conjunto de estructuras aperiódicas. Esto resulta en la predicción de una transición aleatoria de primer orden en la cual no hay una discontinuidad de volumen o entalpía.

La conexión de estos fenómenos con la dinámica viene a través del ritmo en que las estructuras aperiódicas descritas, relajan en configuraciones de equilibrio tipo líquido. Esta relajación es entrópica y es causada por la multiplicidad de estructuras tipo líquido en que el sólido aperiódico se puede transformar. Dado que las relajaciones son heterogéneas, existe una penalización al relajar una gota (de tipo líquido) rodeada de 
mosaicos cristalinos.

De este argumento, surgen numerosas predicciones sobre la dinámica y termodinámica de materiales formadores de vidrios, incluyendo:

- Relación entre fragilidad y discontinuidad en la capacidad calorifica $c_{P}$

- Dependencia de T en el tamaño de las regiones de reacomodamiento cooperativo

- Relación entre fragilidad y la dinámica de relajación de exponencial estirada

- Divergencia de tiempos de relajación $\left(y S_{C} \rightarrow 0\right)$ para una temperatura $0>T_{0} \equiv$ $\mathrm{T}_{\mathrm{K}}$

- No exponencialidad en sus relajaciones

\subsection{Modelos de Vidrio de Retículo}

Suele suceder, que a medida que un modelo busca mejorar su representación de la realidad, este requiere mas parámetros y detalles específicos. Si bien esta complejización puede acercarnos (a veces solo un poco) más a la realidad, nos aleja de una performance rápida. Es por eso que seleccionar los elementos fundamentales que dictaminan un comportamiento y deshacerse del resto no es tarea fácil. Los modelos que priorizan solo algunos elementos clave son llamados modelos mínimos. Estos suelen ser modelos sencillos, en dos o tres dimensiones, que logran captar las características claves del fenómeno en estudio y a la vez nos brindan una compresión cualitativa del fenómeno. Una reducción adicional sobre la complejidad, se obtiene al trabajar sobre un retículo (del ingles lattice) en vez de sobre el continuo. Una característica clave del retículo, es que los sitios que las partículas pueden ocupar están definidos y son numerables.

Kob y colaboradores [Kob and Andersen, 1993], presentan evidencia de un modelo de gas de red cinético para líquido atómico, el cual presenta formación de jaula para alta densidad, en la cual el sistema experimenta una transición dinámica de comportamiento ergódico a no ergódico a medida que la densidad aumenta. Para estos resultados, pocas de las predicciones de MCT valen, a pesar de que el modelo fue diseñado para incorporar los efectos de jaula dinámica que MCT predice. Este hecho, resulta una evidencia de que MCT no parece proveer una descripción correcta de una transición ideal vítrea inducida por comportamiento de jaula, como la del modelo estudiado por Kob y colaboradores.

En nuestro caso, estamos lidiando con heterogeneidades que desencadenan el comportamiento vítreo. Existen dos tipos populares de modelos de grano grueso que buscan presentar estos fenómenos. Por un lado, están los modelos Cinéticamente Cons- 
treñidos (KCM) [Ritort and Sollich, 2003], concentrados en efectos cinéticos como los únicos causantes de la vitrificación. Por otra parte los modelos de Vidrio de Retículo (LGM) [Kob and Andersen, 1993, McCullagh et al., 2005, Pica Ciamarra et al., 2003a, Biroli and Mézard, 2001], proponen que los comportamiento cinéticos son causados por efectos de la termodinámica de las configuraciones, donde las partículas viven en un retículo con reglas globales para su movimiento. En [Garrahan and Chandler, 2002], Garrahan y colaboradores muestran como las heterogeneidades dinámicas en sistemas formadores de vidrio emergen como una consecuencia de las restricciones dinámicas. Ofreciendo además una interpretación de la transición vítrea como una crisis de entropía en el espacio de trayectorias (espacio-tiempo) en vez de el espacio de configuraciones.

Una diferencia saliente entre KCM y LGM viene en que, para los KCM la ralentización viene a través de defectos. En KCM la dinámica es compleja, pero la termodinámica es trivial, pues todas las configuraciones son igualmente probables.

Por otra parte, los LGM no se ralentizan debido a la presencia de ciertos defectos, sino que todas las partículas deben seguir reglas (o vínculos) globales. Esto causa que al aumentar la densidad del sistema disminuya la cantidad de configuraciones distintas para las cuales estas constricciones globales se satisfacen. De esta manera, es la entropía de las configuraciones la que gobierna la ralentización de la dinámica. Además, los LGM tienen la característica de que una dinámica Monte Carlo reproduce las características de dinámica heterogénea observadas en simulaciones newtoneanas de dinámica molecular, pues se basan en realizar movidas configuracionalmente permitidas [Binder et al., 1999].

Nuestro interés estará puesto en en el estudio de modelos LGM que viven en un retículo tridimensional. Los modelos que utilizaremos como líquidos formadores de vidrios son el PCTCC y t154, los cuales desarrollaremos en sus capítulos pertinentes.

\subsection{Objetivos de Este Trabajo}

A pesar de todo el contenido desarrollado en esta introducción, aún queda en pie, quizás, la pregunta mas importante de todas: Causalidad. Aún en ausencia de una transición vítrea ideal (termodinámica), el problema de la ralentización en la relajación estructural, ¿es debido a un fenómeno cinético o termodinámico?

Identificar pruebas irrevocables para determinar si la transición vítrea en un sistema está causada por cuestiones entropicas o puramente por restricciones cinéticas, seria un avance teórico monumental.

En nuestro caso, nuestros esfuerzos serán destinados a objetivos más modestos. 
Las simulaciones computacionales hacen posible explorar distintos modelos de sistemas clásicos de muchas partículas. Sin embargo, y a pesar de la creciente potencia computacional, estos estudios generalmente se encuentran limitados a escalas de tiempo cortas y/o número de partículas pequeño, en comparación con sistemas experimentales.

En un esfuerzo por estudiar tiempos largos y sistemas de tamaño mas ambicioso, hemos optado por estudiar dos modelos mínimos (PCTCC y t154), que en su simplicidad, y mediante técnicas de simulación rápidas que describiremos en el próximo capitulo, nos permiten estudiar la presencia (o ausencia) de fenomenología vítrea en estos modelos y en uno de ellos ( 1154 ), explorar argumentos propuestos por RFOT, en la búsqueda de una longitud estática creciente $\xi(T)$ para temperatura decreciente (o densidad $\rho$ creciente), así como un comportamiento no exponencial para las correlaciones dinámicas.

Estudiaremos también (t154) el comportamiento del tiempo de relajación de sistemas pequeños y confinados con condiciones de contorno amorfas, buscando indicios del mecanismo de relajación para sistemas confinados y su relación con los bordes. 


\section{Capítulo 3}

\section{Simulaciones I: Metodología}

\section{Utilizada en el Estudio Modelos}

de Retículo para Líquidos

\section{Sobreenfriados}

\subsection{Simulaciones Computacionales}

Se puede pensar que gran parte de la educación universitaria en física, es un gran repositorio de problemas altamente idealizados, con una solución cerrada, fruto de una manipulación elegante de expresiones matemáticas [Rapaport, 2004]. Suele haber un abismo entre el planteo que hace una teoría y la extracción de información cuantitativa que pueda ser útil para interpretar un experimento. Las soluciones exactas suelen ser la excepción y no la regla. Es por esto que muchas teorías dependen fuertemente de realizar aproximaciones, tanto numéricas como analíticas.

Las simulaciones suplementan a la teoría, pues estas requieren pocas sino nulas aproximaciones analíticas, a expensas de esfuerzos en calculo numérico. Sin embargo, con el abundante poder de calculo presente en la actualidad, las simulaciones computacionales resultan accesibles a cualquier persona con una computadora. La salida (los resultados) de una simulación, debe ser tratada con los mismos métodos estadísticos que en el análisis de un experimento de laboratorio.

En este trabajo utilizaremos dos técnicas:

- El método Monte Carlo cinético (sección 3.4) para la simulación de sistemas discretos para modelos de líquidos sobreenfriados. 
- La dinámica molecular (sección 7.2), para el estudio de sistemas continuos de partículas autopropulsadas en presencia de distintas interacciones.

\subsection{Sistemas Discretos : Retículos}

Un modelo de retículo es un modelo físico definido sobre un espacio discreto. Los modelos de líquidos sobreenfriados que estudiaremos en el presente trabajo son modelos de retículo, donde las posiciones de las partículas no son representadas por numeros reales, sino por un numero natural (celda) en un retículo $\mathrm{N}$-dimensional. Definiendo el volumen de la celda y el de la caja que contiene el sistema a estudiar, todas las posibles celdas (posiciones) a ser utilizadas quedan completamente definidas. Es importante notar que el uso de un retículo no representa una teselación del espacio. Al teselar, se utiliza una forma geométrica repetida sobre todo el espacio, cubriéndolo sin dejar huecos o solapamiento. La discretización del espacio busca simplificar su descripción dejando pre-definidas la gran cantidad, pero numerable, de configuraciones que el sistema puede tomar.

La simplificación que brinda el uso de celdas permite desarrollar algoritmos que resultan ordenes de magnitud mas veloces que si consideráramos un espacio continuo. El ejemplo mas claro de esta aceleración se da en la búsqueda de vecinos de una dada partícula. En los modelos continuos, las posiciones de las partículas son representadas por números reales, de manera que las posiciones de los vecinos deben ser encontradas con algún criterio, antes de poder realizar operaciones con éstos (ver 7). En cambio, para una celda en un retículo tal búsqueda es innecesaria. Las posiciones de las celdas son conocidas en todo momento, por la propia construcción del espacio. De manera que para una dada celda, las posiciones de todos sus vecinos están determinadas y fijadas.

En este marco, aplicaremos el método Monte Carlo sobre sistemas en el espacio discreto.

\subsection{El Método Monte Carlo MC}

Si nuestro deseo es calcular el valor de expectación $\langle X\rangle$ de un observable $X$, como la energía interna o magnetización, debemos calcular sobre todos los estados $\mu$ del sistema la siguiente expresión:

$$
\langle X\rangle=\frac{\sum_{\mu} X_{\mu} e^{-\beta E_{\mu}}}{\sum_{\mu} e^{-\beta E_{\mu}}}
$$


De esta manera pesamos a todos los estados $\mu$ con su propia probabilidad de Boltzmann. Sin embargo, este método solo puede ser aplicado en la práctica en sistemas muy pequeños, donde el tiempo que lleva tratar todos los estados es experimentalmente aceptable. Para sistemas más grandes debemos promediar sobre algún subconjunto de la totalidad de los estados, introduciendo imprecisiones en el cálculo. Utilizaremos el método Monte Carlo (MC) para minimizar esta imprecisión y alcanzar una buena estimación de $\langle X\rangle$.

El método MC funciona eligiendo un subconjunto de estados, aleatoriamente, a partir de una distribución de probabilidad $p_{\mu}$ que definamos. Si tomamos un subconjunto de estados $M=\mu_{1}, \ldots, \mu_{M}$, podemos estimar la cantidad $X$ así:

$$
X_{M}=\frac{\sum_{i=1}^{M} X_{\mu_{i}} p_{\mu_{i}}^{-1} e^{-\beta E_{\mu_{i}}}}{\sum_{j=1}^{M} p_{\mu_{j}}^{-1} e^{-\beta E_{\mu_{j}}}}
$$

Llamamos $X_{M}$ un estimador de $X$. A medida que aumentamos el numero $M$, el estimador se vuelve mas preciso, de manera que si $M \rightarrow \infty$ tenemos $X_{M}=\langle X\rangle$.

Si contamos con una cantidad de estados limitada, la elección de $p_{\mu}$ es un factor clave a determinar. Si tomásemos $p_{\mu}=1 / N$, caso conocido como muestreo simple, no estaríamos discriminando ningún estado sobre otro. Así, las muestras que tomáramos del sistema probablemente no sean representativas de la totalidad del sistema y el estimador $X_{M}$ nos daría un mala estimación de $X$. Si conociésemos de antemano, que estados tienen la contribución mas importante al calculo de $\langle\mathrm{X}\rangle$, entonces podríamos elegir muestrear entre esos estados $M$ y así obtener un buen estimativo de $\mathrm{X}$. Existe una técnica para elegir los estados mas importantes para la buena determinación de $\mathrm{X}$ y se la conoce como muestreo de importancia.

\subsubsection{Muestreo de Importancia}

Tomaremos una muestra de los estados del sistema, de manera que la probabilidad de cada estado sea proporcional a su peso de Boltzmann, es decir, tomamos los estados de manera que la probabilidad de que un cierto estado $\mu$ resulte elegido sea $p_{\mu}=$ $Z^{-1} e^{\beta E_{\mu}}$, donde $Z$ es la función de partición. Resulta así, reemplazando en (3.2):

$$
X_{M}=\frac{1}{M} \sum_{i=1}^{M} X_{\mu_{i}}
$$

De esta manera tenemos un método para estudiar $X_{M}$, eligiendo mas frecuentemente los estados que el sistema ocupa la mayor parte del tiempo, y así, representando mas precisamente el sistema real. 
Sin embargo, falta aún explicar como seleccionaremos los estados de manera que cada uno aparezca con su probabilidad de Boltzmann asociada. Para comprender esta ultima parte, deberemos comprender los procesos de Markov.

\subsubsection{Procesos de Markov}

Un proceso de Markov hace que un sistema que se encuentra en un estado $\mu$ pase a un nuevo estado (de ese sistema) $v^{1}$. Este paso se realiza aleatoriamente, es decir, no siempre partiendo de $\mu$ se llega a $v$ sino que depende una probabilidad de transición $\mathrm{P}(\mu \rightarrow v)$. Para un proceso de Markov, $\mathrm{P}(\mu \rightarrow v)$ debe satisfacer 2 condiciones: (1) No debe variar con el tiempo. (2) Solo debe depender de $\mu$ y $v$, es decir, independiente de la historia de los estados que recorrió el sistema. Además, debe satisfacer la restricción:

$$
\sum_{v} P(\mu \rightarrow v)=1
$$

Durante una simulación MC, se utiliza un proceso de Markov para generar una cadena de Markov de estados. El proceso de Markov se elige, de manera que luego de muchas iteraciones comenzando en cualquier estado, podemos generar una sucesión de estados que aparecen con probabilidades dadas por una distribución de Boltzmann. Diremos que el sistema llega al equilibrio cuando alcanza una distribución de Boltzmann. Para que esto suceda, debemos pedir además que el proceso de Markov cumpla las condiciones de ergodicidad y balance detallado.

\subsubsection{Ergodicidad y Balance Detallado}

La condición de ergodicidad es el requerimiento para nuestro proceso de Markov, que para una simulación suficientemente larga, el sistema pueda recorrer todos ${ }^{2}$ los estados, sin importar el estado inicial. La condición de ergodicidad nos dice que tenemos permitido hacer $\mathrm{P}(\mu \rightarrow \vee)=0$ para ciertos estados $\mu, \nu$, pero que debe existir algún camino de estados intermedios para partir de $\mu$ y llegar a $v$. Si esto no fuese así, existirían estados inaccesibles.

Por último, la condición de balance detallado, es condición suficiente que la distribución de probabilidad que generamos cuando nuestro sistema llega al equilibrio, sea la distribución de Boltzmann y no otra. Esto queda garantizado si, con $p_{\mu}=Z^{-1} e^{\beta E_{\mu}}$,

\footnotetext{
${ }^{1}$ El nuevo estado $v$ puede ser el estado inicial $\mu$.

${ }^{2}$ Tiene que ser posible recorrer todos los estados, aunque el tiempo para hacerlo puede ser ordenes de magnitud mas grande que el tiempo experimental disponible para realizar la simulación.
} 
se satisface que:

$$
p_{\mu} P(\mu \rightarrow \nu)=p_{\nu} P(\nu \rightarrow \mu)
$$

\subsubsection{El Algoritmo de Metropolis}

Podemos elegir cualquier algoritmo que deseemos para generar nuevos estados, manteniendo nuestro conjunto de probabilidades de transición, utilizando una tasa de aceptación. Metropolis es una forma de conseguir un proceso de Markov con balance detallado. Podemos pensarlo, separando la probabilidad de transición en la siguiente manera:

$$
\mathrm{P}(\mu \rightarrow v)=\mathrm{g}(\mu \rightarrow v) \mathrm{A}(\mu \rightarrow v)
$$

Donde $\mathrm{g}(\mu \rightarrow \boldsymbol{v})^{3}$ es la probabilidad de seleccionar un estado, dado un estado inicial $\mu$ que nuestro algoritmo genere un estado objetivo $v$, mientras que $A(\mu \rightarrow v)$ es la tasa de aceptación o probabilidad de aceptación. La tasa de aceptación es la probabilidad de que nuestro sistema pase del estado $\mu$ al $\nu$ o que se quede en $\mu$. Como elegir $A(\mu \rightarrow v)$ es importante, pues si es pequeña la probabilidad de aceptar un cambio de estado, el sistema se quedara quieto y no tendremos evolución.

La elección típica del algoritmo Metropolis para $A(\mu \rightarrow v)$ es:

$$
A(\mu \rightarrow v)= \begin{cases}e^{-\beta\left(E_{v}-E_{\mu}\right)} & \text { si } E_{v}-E_{\mu}>0 \\ 1 & \text { si no }\end{cases}
$$

Donde $E_{\mu}$ representa la energía del estado $\mu$. Es decir, si el nuevo estado posee una energía menor o igual al estado actual, siempre aceptaremos la transición a dicho estado. Ahora, si la energía del nuevo estado es mayor a la del estado actual, existe una probabilidad dada por la exponencial decreciente, de aceptar la transición a dicho estado de cualquier manera. Este algoritmo satisface el balance detallado si $\mathrm{g}(\mu \rightarrow$ $v)=g(v \rightarrow \mu)$ y es quizás uno de los mas populares en todas las simulaciones MC. Denotaremos a simulaciones de este tipo Metropolis Monte Carlo (MMC).

\subsection{El Método Monte Carlo Cinético (KMC)}

El método MMC que presentamos siempre es correcto, pero no siempre resulta eficiente. Hay sistemas en los que la gran mayoría de las propuestas de movidas que

\footnotetext{
${ }^{3} \mathrm{~g}(\mu \rightarrow v)$ es un parámetro libre del método y debe ser ajustado al problema especifico a tratar.
} 
realiza MMC serán rechazadas. Llevando a que el sistema evolucione de manera infrecuente a pesar del gran esfuerzo computacional. Esto significa, que durante el tiempo experimental disponible, el sistema rara vez visite otros estados. Esto es un problema desde el punto de vista experimental, dado que para ver que el sistema evoluciona, necesitaríamos una cantidad de pasos Monte Carlo demasiado grande, demorando un tiempo (en algunos casos) ordenes de magnitud mayor al disponible. Esto nos lleva a pensar que debemos encontrar otro enfoque, donde podamos lidiar con sistemas bloqueados en tiempos mas cortos. Una herramienta para este tipo de problemas donde los eventos son infrecuentes son las simulaciones Monte Carlo Cinético (KMC).

Notamos que KMC sigue un proceso de Markov con espacio discreto, pero tiempo continuo. Veremos en la próxima sección que el tiempo transcurrido entre estados durante la evolución del sistema no es constante, sino que sera calculado para cada paso.

\subsubsection{Sistemas de Eventos Improbables y Perdida de Ergodicidad}

Un sistema de eventos infrecuentes se caracteriza por tener una dinámica de transiciones ocasionales de un estado a otro, seguido de largos periodos de inactividad entre dichas transiciones [Voter, 2007]. Para un sistema en el continuo, podemos pensar al sistema bloqueado, como si este (en su totalidad) se encuentra en un pozo de mínima energía, sin embargo, próximo al pozo y con una barrera de energía por medio, hay otros pozos a los que el sistema puede ir (Esquematizado en figura 2.4)

Como mencionamos en 2.6.1, si movemos el sistema de un pozo a otro, no estamos simplemente moviendo algunas pocas partículas de un lugar a otro, sino al sistema completo, como un todo, de un estado a otro. Dado que el sistema pasa tanto tiempo en estos mínimos, este se olvida como llego a dicho mínimo en primera instancia, es decir, la probabilidad de escapar del mínimo solo depende del estado actual y estado objetivo, no de la historia del sistema. Esto es una característica típica de una cadena de Markov. De manera que si nos encontramos en un estado inicial $i$ y vamos a un estado final $\mathfrak{j}$, realizando aproximaciones, habrá involucrada una probabilidad $k_{i j}$, de manera que para cada camino que el sistema recorra, tendremos un conjunto de $k_{i j}$.

Consideremos el caso de un sistema en el retículo con muchas restricciones. Si nos encontramos en un estado $i$, puesto que nos encontramos en un retículo, sabemos que existe una cantidad limitada de posibles operaciones que el sistema puede realizar. Esto significa que para cada estado $i$, podemos elaborar una lista (sin aproximaciones) con todos los posibles estados $j$ a los que el sistema puede moverse. A medida que el sistema es sujeto a mas restricciones, menor sera el tamaño de la lista de movidas 

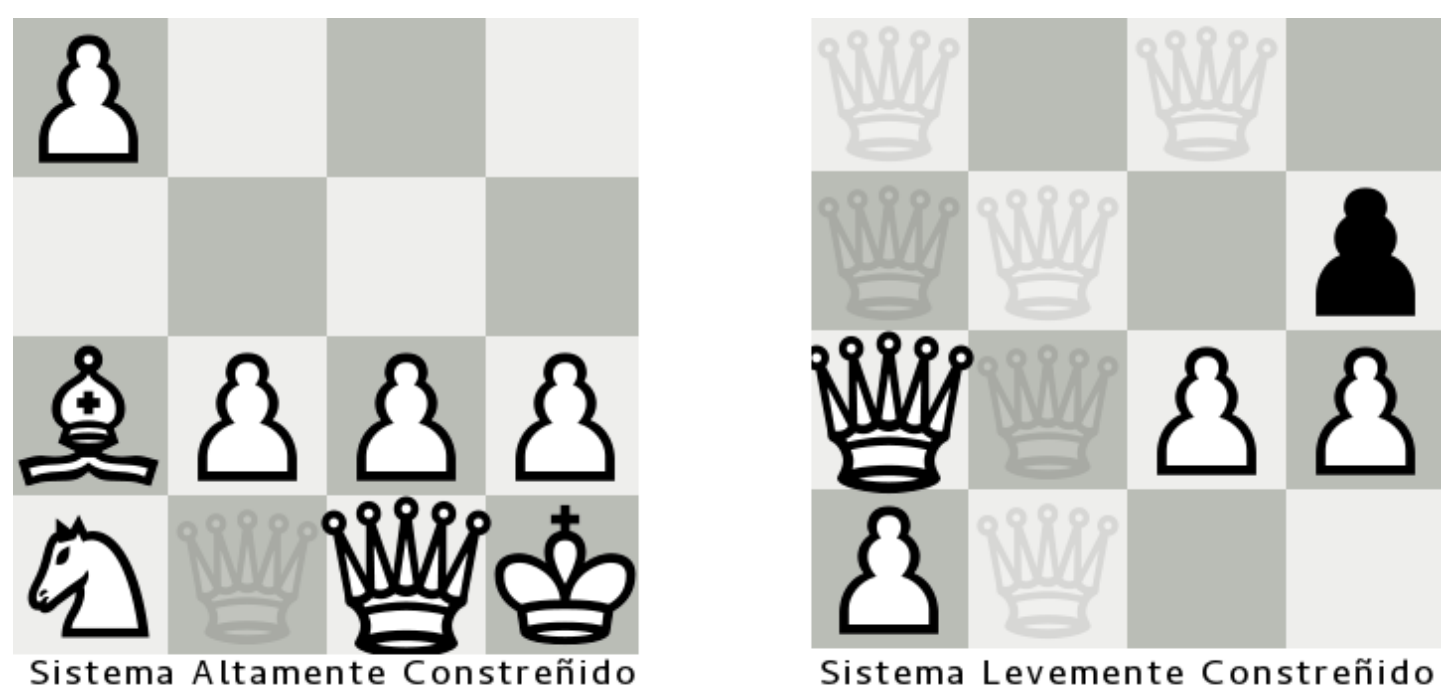

Figura 3.1: Esquematización de los posibles estados j a los que un sistema en el estado $i$ puede evolucionar. En este ejemplo consideramos los posibles movimientos de la reina en un juego de ajedrez. Notamos que un sistema fuertemente constreñido posee una menor cantidad de estados $\mathfrak{j}$ disponibles, mientras que si se eliminan restricciones, el número de posibles estados $\mathfrak{j}$ aumenta.

posibles. Esquematizamos esta idea, dando el ejemplo de dos estados en un juego de ajedrez (ver figura 3.1). Podemos pensar que una persona que sabe las reglas, juega al ajedrez en el estilo KMC, solamente considerando movidas legales. Alguien que no conoce las reglas, intentara ubicar la partícula en cualquier posición (incluso posiciones ocupadas o inaccesibles) y su intento sera rechazado. Como sucede en el MMC.

La característica principal de la simulación Monte Carlo Cinética, es que esta evalúa los conjuntos de $k_{i j}$ en que el sistema se encuentra y calcula la probabilidad que tiene el sistema de avanzar a un nuevo estado $j$ a partir de $i$. En vez de realizar intentos de movida aleatorios, que en su mayoría serán fallidos, KMC no propone, sino que directamente realiza una movida permitida. $K_{\text {tot }}$ representa la probabilidad total de escapar de un cierto estado $i=0$ a cualquier otro estado disponible $i \neq 0$ donde:

$$
K_{\text {tot }}=\sum_{i \neq 0} k_{i}
$$

Luego de realizar una movida, el tiempo sera incrementado en un valor $\Delta \mathrm{t}$ de manera inversamente proporcional a $K_{\text {tot }}$, usando un número aleatorio $x$ uniformemente distribuido en $(0,1]$ y una distribución exponencial [Voter, 2007]:

$$
\Delta \mathrm{t}=\frac{1}{\mathrm{~K}_{\text {tot }}} \exp \left(-\mathrm{x} / \mathrm{K}_{\text {tot }}\right)
$$


De esta manera, los sistemas mas constreñidos, demoran menos tiempo de calculo computacional para avanzar un mismo tiempo experimental. Esta propiedad resulta de tremendo interés para estudiar sistemas vítreos, los cuales se caracterizan por tener una evolución que se enlentece exponencialmente a medida que variamos parámetros del sistema. Volveremos sobre esto en la sección 4.2.

\subsection{Herramientas Complementarias}

En esta sección llevamos nuestra atención a dos técnicas que utilizaremos en partes especificas de los estudios de sistemas vítreos.

Una técnica discutida por [Albano et al., 2011], para estudiar la dinámica de tiempos cortos. Notamos que únicamente utilizaremos esta técnica en un estudio especifico del modelo PCTCC en la sección 4.5.

La otra, es la prueba BIC [Cavagna et al., 2007]. Esta es una herramienta para estudiar el estacionamiento de la relajación de sistemas confinados que presentan dinámica lenta. Sera utilizada en la sección 5.4. En la sección de materia activa 8.3, usamos la prueba BIC como inspiración para realizar una prueba de condición inicial.

\subsubsection{Dinámica de Tiempos Cortos}

La técnica analiza las series temporales de un conjunto de observables, donde los parámetros de control (por ejemplo, temperatura o campo magnético) se encuentran fijados en sus valores de transición. Estos observables son típicamente el parámetro de orden y algunos de sus momentos o cumulantes. El análisis requiere que la configuración inicial sea controlada cuidadosamente, aveces fijando precisamente el valor inicial de dicho parámetro de orden.

En una gran variedad de Hamiltonianos y sistemas dinámicos, luego de un tiempo microscópico, la evolución temporal de los observables en el punto critico sigue una ley de potencia. Esto es significativo, dado que indica que un enfoque de tiempos cortos puede ser utilizado para identificar valores críticos de los parámetros de control.

Dado que los exponentes universales de una transición pueden ser directamente relacionados a los de una ley de potencia de los observables antes mencionados. Obtener los exponentes universales, junto con los parámetros de orden críticos es uno de los objetivos centrales de esta técnica.

Como menciona [Loscar et al., 2009], si nuestro interés es aplicar este método para la determinación de espinodales debemos ser cuidadosos. Estrictamente, el concepto de espinodal esta definido rigurosamente para sistemas con rango infinito de inter- 
acción (campo medio). En sistemas de corto rango, una pseudoespinodal puede ser definida por extrapolación de medidas metaestables, pero donde el punto en si mismo no puede ser alcanzado, dado que yace mas allá del limite de metaestabilidad. Sin embargo, [Loscar et al., 2009] muestran que existe un buen acuerdo con el punto espinodal termodinámico de modelos de campo medio y el punto pseudoespinodal obtenido de la extrapolación del equilibrio metaestable. De esta manera, podemos realizar una determinación del punto espinodal sin preocuparnos por problemas de equilibración determinando puntos espinodales mediante dinámica de tiempos cortos.

\section{La Técnica de Dinámica de Tiempos Cortos (DTC)}

El procedimiento consiste en buscar una ley de potencia de relajación temporal, partiendo de un estado inicial preparado con una cierta prescripción. Para puntos críticos de equilibrio, el régimen de ley de potencia se mantiene por un tiempo que aumenta con el tamaño del sistema, pero para el caso de espinodales, este régimen se encuentra solo para un intervalo finito [Loscar et al., 2009].

El procedimiento es el siguiente:

- Preparar una muestra equilibrada a alta temperatura. Este sera el estado inicial desordenado.

- Al tiempo $t=0$ realizar un enfriamiento súbito a una temperatura por debajo de la fase desordenada.

- Permitir al sistema relajar mientras se recopila el parámetro de orden y sus fluctuaciones hasta un tiempo típicamente muy inferior al de relajación del sistema.

- Buscar comportamiento de ley de potencia. La temperatura espinodal queda determinada como la temperatura a la cual la ley de potencia se dura su máximo.

- Extraer exponentes de la ley de potencia mediante regresión.

\subsubsection{Prueba BIC}

En el estudio de equilibración de sistemas de partículas, podemos preguntarnos cómo verificar que cuando el autosolapamiento (ver sección 2.3.10) decae a un valor estacionario, ese valor es un valor termalizado. Para sortear este problema, podemos utilizar una prueba BIC. En su trabajo [Cavagna et al., 2007], Cavagna y colaboradores describen la prueba de condición inicial $\beta$ (BIC), como una herramienta para verificar si el autosolapamiento asintótico alcanza el valor de equilibrio. La motivación se origina en 
I.

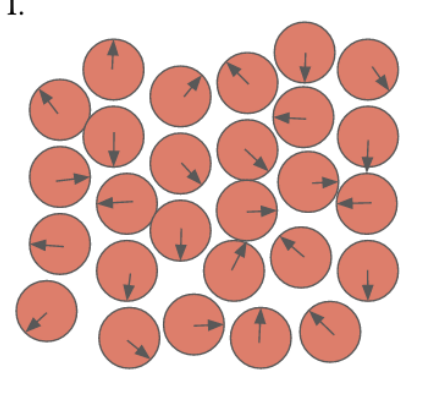

II.

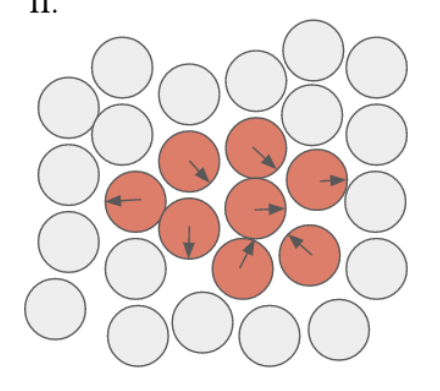

III.

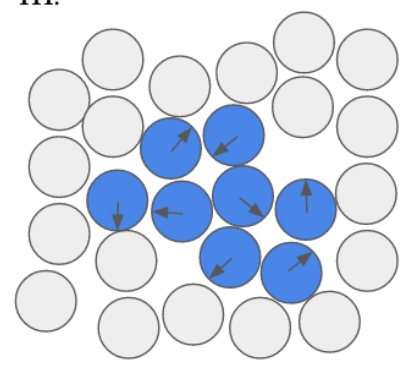

Figura 3.2: Caricatura de los pasos en una prueba BIC. Primero se toma una configuración de partículas. Luego se define un exterior y un interior, donde el exterior queda inmovilizado y define la cavidad. Finalmente se reemplaza el interior de la cavidad, por un nuevo conjunto de partículas con autosolapamiento nulo respecto a las particulas removidas.

la necesidad de asegurarse que las simulaciones son de una duración suficiente como para alcanzar el valor de equilibrio o bien de verificar que el equilibrio no puede alcanzarse en el tiempo disponible.

Esta prueba resulta de interés para el estudio de relajación ante condiciones de contorno amorfas ( $A B C$, ver sección 2.5). En este caso no conocemos a priori el valor termalizado de cada configuración y deseamos verificar que el sistema lo ha alcanzado (y cuanto vale).

Las ABC que utilizaremos consisten en dividir al sistema de partículas en dos partes: Una parte es la externa, la cual quedara congelada, en el sentido de que no podrá mover o cambiar sus partículas de ninguna manera. La otra parte es la interna, para la cual las partículas son libres de moverse, pero quedan confinadas por la parte externa. De esta manera queda definida una cavidad de tamaño arbitrario donde las partículas son libres de moverse.

La idea de la prueba BIC es inicializar la cavidad en una cierta configuración $\beta$ la cual tiene un autosolapamiento nulo con otra configuración $\alpha$ usada para termalizar el sistema y que esta fijada en la condición de contorno. Esquematizamos esta situación en la figura 3.2.

De esta manera, el autosolapamiento $q_{\alpha \beta}(t)$ es nulo a tiempo $t=0$ y debe aumentar al mismo valor asintótico que el del autosolapamiento estandar $q_{\alpha \alpha}(t)$. Si la cavidad es capaz de termalizar, ambos autosolapamientos $\left(q_{\alpha \beta}(t)\right.$ y $\left.q_{\alpha \alpha}(t)\right)$ deben encontrarse en el mismo valor de equilibrio y la prueba resulta positiva. Si los autosolapamientos se vuelven asintóticos sin antes encontrarse o se agota el tiempo de simulación antes que los autosolapamientos se encuentren, diremos que la prueba BIC es negativa y el 
sistema no ha termalizado.

Esta prueba resulta muy simple de realizar en el caso de simulaciones de modelos en el retículo, pues basta definir la cavidad mediante celdas con movilidad y celdas inmovilizadas. En el caso en que se utiliza una dinámica gran canónica, si se vacía la cavidad luego de tomar la configuración inicial de referencia, el autosolapamiento inicial sera nulo. A medida que se creen nuevas partículas dentro de la cavidad, el autosolapamiento crecerá, permitiéndonos efectuar la prueba fácilmente. 


\section{Capítulo 4}

\section{Modelo PCTCC}

Un modelo mínimo, opuesto a un modelo realista, debe ser capaz de exhibir fenomenología vítrea y a la vez permitir un estudio teórico simplificado y veloz de computar mediante una simulación numérica. Debido a la dinámica lenta propia de los vidrios, los tiempos de simulación requeridos para alcanzar la termalización, muchas veces resultan ser mayores que los tiempos de cómputo disponibles. Puesto que para las temperaturas donde se realizarían las observaciones más interesantes, el tiempo para alcanzar la termalización es alto, es sumamente importante contar con un modelo veloz de simular.

Es por eso que consideramos el modelo de vidrio de retículo propuesto por Pica Ciamarra y colaboradores [Pica Ciamarra et al., 2003a] [Pica Ciamarra et al., 2003b], al que llamaremos PCTCC ${ }^{1}$. Este modelo además de poseer una interpretación geométrica sencilla, resulta atractivo, tanto teóricamente por la posibilidad de usar el retículo de Bethe [Rivoire et al., 2004], así como numéricamente por la posibilidad de estudiarlo utilizando el metodo Monte Carlo sin necesidad hacer uso de aproximaciones.

Desde el punto de vista vítreo, Pica Ciamarra y colaboradores detectan la presencia de efecto jaula y la presencia de dos escalas temporales en las funciones de relajación (Discutido en sección 2.3). Además, encuentran que el modelo no tiene tendencia a cristalizar, aun para ritmos de enfriamiento sumamente lentos.

En este capitulo, presentaremos el modelo PCTCC (sección 4.1), luego haremos una discusión referente al uso de los algoritmos MMC y KMC (sección 4.2), despues realizaremos un estudio de la metaestabilidad (sección 4.3) y luego una determinación de sus límites (sección 4.4). Después, mediante el uso de la dínamica de tiempos cortos extraeremos una cota para la temperatura espinodal (sección 4.5) y finalmente realizaremos un estudio de envejecimiento para determinar las capacidades del modelo para ser un líquido formador de vidrios (sección 4.6).

\footnotetext{
${ }^{1}$ Algunos resultados expuestos en este capitulo fueron publicados en [Seif et al., 2015].
} 

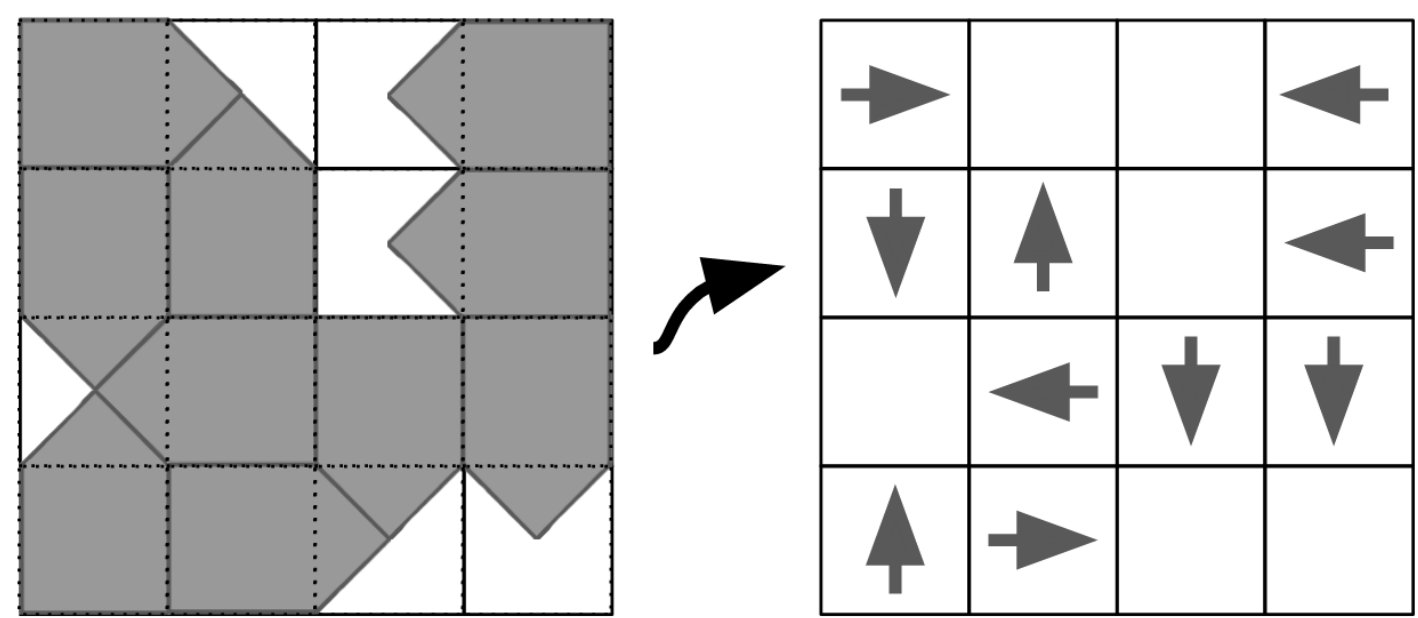

Figura 4.1: Caricatura del modelo de retículo PCTCC para un caso bidimensional inspirado en partículas que excluyen volumen adyacente según su orientación. Se observa que los spines únicamente apuntan a sitios vacíos.

\subsection{Modelo PCTCC}

Podemos formular al modelo de la siguiente manera:

Partículas clásicas con una orientación (spin) son colocadas en un retículo cubico de lado L, donde cada sitio $\vec{R}$ tiene un numero de ocupación $n_{R}=0,1$. En cada sitio ocupado, $\vec{\sigma}_{R}$ representa la orientación de la partícula hacia uno de sus primeros seis vecinos. Recalcamos que hay exclusión, de manera que en cada $\vec{R}$ solo 0 o 1 partícula puede estar alojada y $\vec{\sigma}_{R}$ debe apuntar hacia un sitio $\vec{R}$ vacio $\left(n_{R}=0\right)$. Esto puede resumirse en:

$$
\vec{R}+\vec{\sigma}_{R}=\vec{R}^{\prime} \Longleftrightarrow n_{R^{\prime}}=0
$$

Por analogía puede pensarse que cada partícula no cabe en un sitio y ocupa una parte de un sitio contiguo (al que apunta), dejándolo inhabilitado para ser ocupado por otra partícula. Mostramos una caricatura del sistema en 4.1. Se define una dinámica local, donde las partículas realizan roto+translaciones a primeros vecinos, es decir, las partículas pueden rotar y/o desplazarse hacia un sitio vació entre sus primeros vecinos en una única operación.

Estudiamos 2 tipos de conjuntos:

- Un conjunto canónico, donde el numero de spines (partículas) se encuentra fijo y el sistema solo puede re-acomodarse de acuerdo a los sitios vacíos y las movidas roto+translacionales.

- Un conjunto gran canónico, en el que el sistema se encuentra en contacto con un 
baño de partículas, que permite crear y destruir spines en distintos sitios, además de moverlos como el conjunto canónico.

Para el conjunto canónico no hay escala de energía $(E=0$ o $E=\infty)$, por lo tanto, la temperatura no es una variable relevante y no esta involucrada en ningún paso de la dinámica ${ }^{2}$. Para el caso del conjunto canónico, la única variable relevante sera la densidad $\rho$.

Para el conjunto gran canónico, además de la dinámica roto+translacional del ensamble canónico, contamos con un baño de partículas caracterizado por $\alpha$ que creará y destruirá partículas. Puesto que a través de $\alpha=\beta \mu=\frac{\mu}{k_{B} T}$ controlamos $\rho$, podemos fijar $k_{B}=1$ y trabajar con temperatura $T$ en términos de $\mu$. Mas adelante, veremos que la probabilidad de destruir una partícula depende de $\alpha$. La dinámica gran canónica produce que el sistema no se atasque tan seguido como cuando se utiliza el conjunto canónico, dado que cuando las partículas no tengan movidas roto+translacionales disponibles, existe la posibilidad de que una de ellas sea destruida y aparezcan nuevas movidas permitidas para las demás.

Puesto que no se busca reproducir cuantitativamente ningún resultado experimental de laboratorio, la unidad de tiempo utilizada sera un paso Monte Carlo. Nos referiremos a pasos Monte Carlo o tiempo de manera indistinta.

Si bien comenzaremos las simulaciones con el retículo vacío, utilizando una dinámica gran canónica para crear partículas en los sitios, puede ser de interés, comenzar la simulación con un cristal perfecto (máxima densidad). En [Pica Ciamarra et al., 2003a] se provee la receta para generar un cristal perfecto, que sigue la siguiente formula:

Sea a un numero entero y $x, y, z$ las coordenadas espaciales en las que puede ubicarse el spin. Si calculamos $a=(x+2 y+3 z \bmod 7)$.

Si $a=0$ se deja el sitio vacío, si $a=1,2,3$ se coloca un spin en dirección negativa $(-1)$ en dirección $x, y, z$ respectivamente. Si $a=4,5,6$ se coloca un spin en dirección positiva (1) en dirección $z, y, x$ respectivamente. Utilizando esta receta, se logra un estado cristalino puro con una densidad de $\rho=6 / 7 \simeq 0.857$

Finalmente, como manera de cuantificar la cantidad de fase cristalina presente en una dada muestra, definimos la fracción de masa cristalina m, como la fracción de sitios vacíos rodeados de seis partículas apuntando hacia ellos ${ }^{3}$. Esta cantidad resulta muy fácil de evaluar y da una medida de la cantidad de cristal presente, independiente de el

\footnotetext{
${ }^{2}$ Esto resulta así por que la diferencia de energía entre un estado permitido y uno prohibido es infinito, de manera que si analizamos la expresión (3.7) vemos que siempre se rechazara una movida prohibida, sin importar cuanto valga $\beta \mu \simeq \mu / \mathrm{T}$.

${ }^{3}$ La única manera de que los sitios vacíos aparecen en un cristal perfecto es cuando todos sus vecinos apuntan hacia el.
} 
tamaño de los dominios. Notamos que m no es un correcto parámetro de orden, pues no se anula en la fase liquida, pero vemos que incrementa su valor fuertemente cuando el sistema comienza a cristalizar. Es por eso que m es una medida útil para estudiar el comienzo de la cristalización.

\subsection{Comparación entre Monte Carlo Metropolis y Monte Carlo Cinético}

Nuestro interés por el comportamiento sobreenfriado, nos lleva a estudiar sistemas de alta densidad, donde el modelo evoluciona lentamente debido a que el numero de acciones permitidas es muy pequeño. Por acción nos referimos a cualquier transición entre estados propuesta por el algoritmo Monte Carlo; en el caso gran canónico esto incluye la creación y aniquilación de partículas. Bajo estas condiciones, el algoritmo Monte Carlo Metropolis (MMC) resulta muy ineficiente, dada su gran tasa de rechazos para alta densidad. Es por este motivo que, para acelerar los tiempos de simulación optamos por el algoritmo Monte Carlo Cinetico (KMC) discutido previamente en la sección 3.4.

A fin de tener una verificación cuantitativa sobre los tiempos de computo involucrados usando ambos algoritmos, realizamos simulaciones para los mismos conjuntos de parámetros usando los distintos algoritmos. Verificamos que los resultados producidos por MMC y KMC resultan indistinguibles, como se ve en la figura 4.2.

Observamos 3 regiones de $\mathrm{T} / \mu$ en las que podemos dividir el análisis de los algoritmos:

- $\mathrm{T} / \mu>\frac{1}{3}$ la simulación KMC demora órdenes de magnitud más que MC. El procedimiento KMC de generar y mantener listas de movidas permitidas resulta menos eficiente que sortear propuestas de movidas con el método MC.

- $\frac{1}{5}<\mathrm{T} / \mu<\frac{1}{3}$ Ambos algoritmos demoran tiempos comparables.

- $\mathrm{T} / \mu<\frac{1}{5} \mathrm{KMC}$ resulta ordenes de magnitud mas rápido que MC.

Nuestro interés esta puesto alrededor de la zona $T / \mu \in\left[\frac{1}{12}, \frac{1}{5}\right]$, de manera que seguiremos adelante únicamente utilizando el algoritmo KMC. La variable de control es $\mathrm{T} / \mu$, por simplicidad a lo largo del trabajo nos referiremos a esta cantidad como temperatura, realizando aclaraciones entre $\mathrm{T}$ y $\mathrm{T} / \mu$ donde corresponda. 


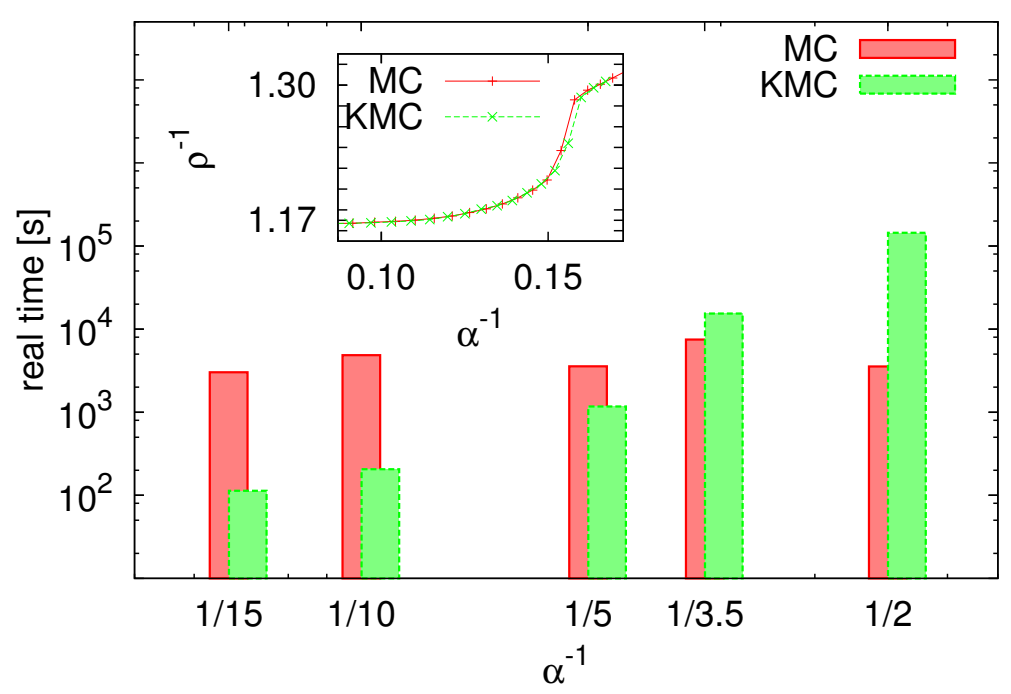

Figura 4.2: Comparación entre el tiempo real de simulación entre simulaciones Monte Carlo Metropolis (MMC) y Monte Carlo Cinético (KMC). Se observa como el tiempo de computo KMC es fuertemente dependiente de la densidad. Recuadro: Presentamos Densidad Inversa $\rho^{-1}$ vs $T / \mu$ para el calentamiento de un cristal perfecto. Comparamos un proceso de calentamiento $\dot{\mathrm{T}} / \mathrm{T}=10^{-8}$, utilizando los mismos parámetros, pero comparando MC con KMC. Observamos buen acuerdo entre los comportamientos.

\subsection{Estudio de Metaestabilidad}

Como discute [Pica Ciamarra et al., 2003a], si preparamos una configuración inicial de cristal perfecto podemos determinar la temperatura de fusión $\mathrm{T}_{\mathrm{m}}$ calentándolo lentamente con distintos ritmos $\dot{T}$ hasta que se produce un cambio súbito en su densidad. Como muestra la figura 4.3, determinamos $\mathrm{T}_{\mathrm{m}}=0.15 \mu$, verificando el resultado hallado por [Pica Ciamarra et al., 2003a]. En contraste, cuando realizamos enfriamientos observamos que el sistema no cristaliza, sino que el sistema se mantiene en un régimen de líquido sobreenfriado hasta que finalmente se sale del equilibrio a una temperatura dependiente del ritmo de enfriamiento.

Otra medida que podemos extraer de los enfriamientos, es la extrapolación (linea punteada en la figura 4.3) de la región de sobre-enfriamiento de $\rho^{-1}$ hasta que intersecta el cristal. Esto arroja un valor de $T_{K} \simeq 0.05 \mu$, valor que podemos utilizar para estimar la temperatura de Kauzmann.

Utilizaremos correlaciones dinámicas (ver sección 2.3.10), en particular el autosolapamiento $Q(t)$, para estudiar la relajación del sistema. Partiendo desde un tiempo inicial 


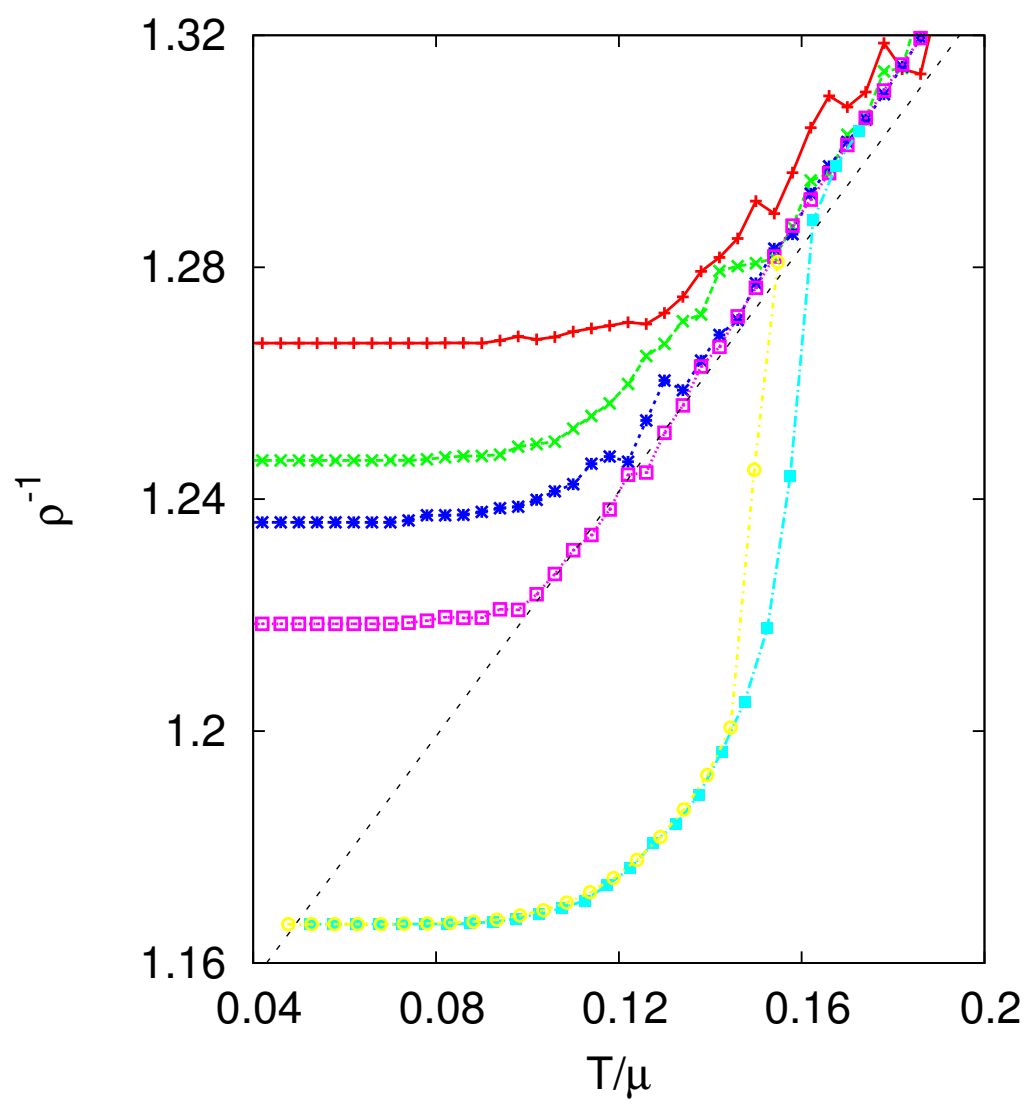

Figura 4.3: $\rho^{-1}$ como una función de $\mathrm{T}$ para distintos ritmos de enfriamiento. Para $\dot{T} / \mathrm{T}=-10^{-5}$ (rojo), el sistema rápidamente sale de equilibrio, mientras que para $\dot{\mathrm{T}} / \mathrm{T}=-10^{-6}$ (verde), $\dot{\mathrm{T}} / \mathrm{T}=-10^{-7}$ (azul) y $\dot{\mathrm{T}} / \mathrm{T}=-10^{-8}$ (purpura) el sistema mantiene un equilibrio metaestable de liquido sobreenfriado que puede ser hallado a temperaturas progresivamente mas bajas. El calentamiento del cristal puede ser utilizado para estimar $\mathrm{T}_{\mathrm{m}}$, como el que se muestra en cyan $\left(\dot{\mathrm{T}} / \mathrm{T}=10^{-8}\right)$ y amarillo $\left(\dot{\mathrm{T}} / \mathrm{T}=10^{-9}\right)$, determinando $T_{m}=0.15 \mu$ de este ultimo. 


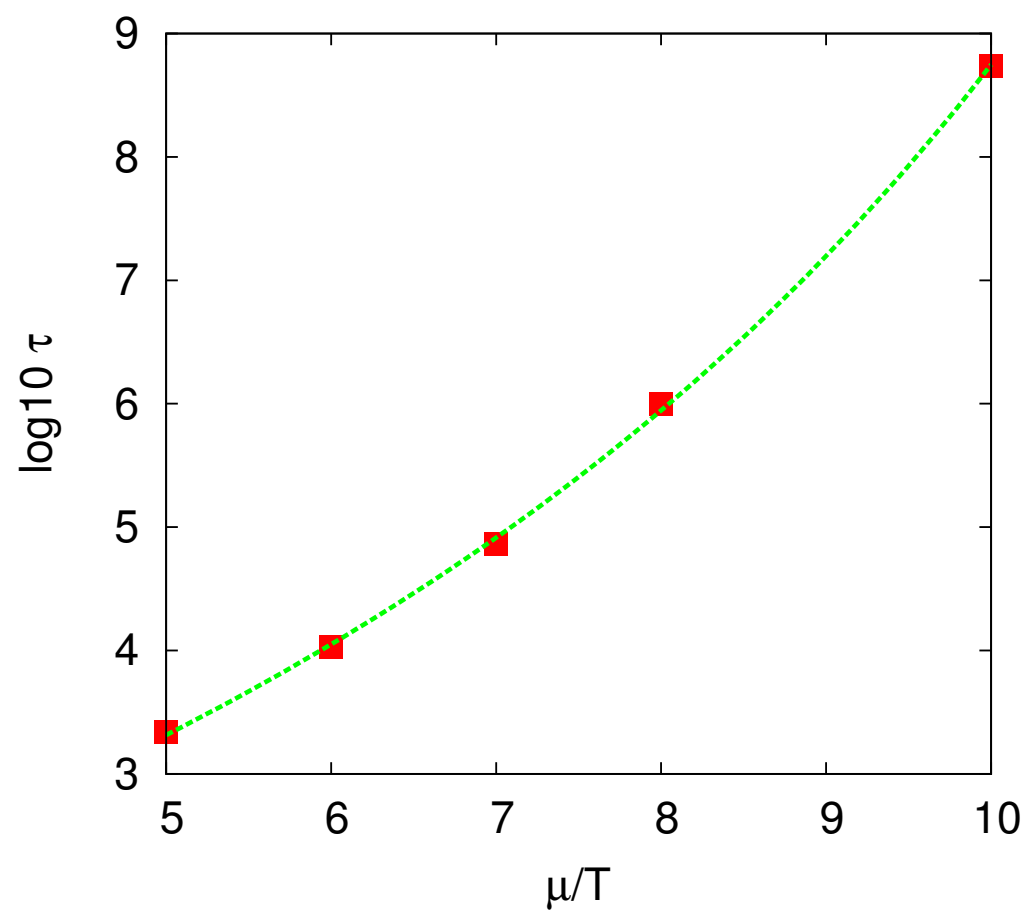

Figura 4.4: Gráfico Angell mostrando un comportamiento no-Arrhenius. La linea continua representa el ajuste VFT para un valor de $T_{0}=0.048 \mu$.

$t_{0}=0$, para este modelo el autosolapamiento Q se define [Pica Ciamarra et al., 2003a]:

$$
Q(t)=\frac{1}{N} \sum_{i}^{N}\left\langle n_{i}(0) n_{i}(t)\left(\vec{\sigma}_{i}(0) \cdot \vec{\sigma}_{i}(t)\right)\right\rangle,
$$

Notamos que $\mathrm{n}$ mide la ocupación del sitio, mientras que $\vec{\sigma}$ mide la orientación de las partículas en dicho sitio. De esta manera, aun si las partículas se mantienen en la misma posición, pero apuntan en direcciones distintas a las originales, $\mathrm{Q}(\mathrm{t})$ disminuirá. Nos interesa extraer un tiempo de relajación $\tau_{R}$ a partir de $Q(t)$. Para ello, ajustamos $Q(t)$ con una exponencial estirada (relajación Kolrausch-William-Watts [Ediger et al., 1996, Lindsey and Patterson, 1980]) $e^{-\left(t / \tau_{R}\right)^{\beta}}$.

Como mencionamos en la sección 2.3.4, podemos obtener una medida de $T_{K}$ realizando un ajuste de la ley de Vogel-Fuchter-Tamman usando $T$ y $\tau_{R}$, como muestra la figura 4.4 usando:

$$
\tau_{R}=\tau_{0} \exp \left[\mathrm{B} /\left(\mathrm{T}-\mathrm{T}_{0}\right)\right]
$$

El ajuste arroja un valor de $\mathrm{T}_{0}=0.048 \mu$, muy cercano al $\mathrm{T}_{\mathrm{K}}$ estimado mediante la extrapolación. El incremento dramático del tiempo de relajación $\tau_{R}$ para pequeñas variaciones de la temperatura que observamos, es típico de la fenomenología vítrea. 


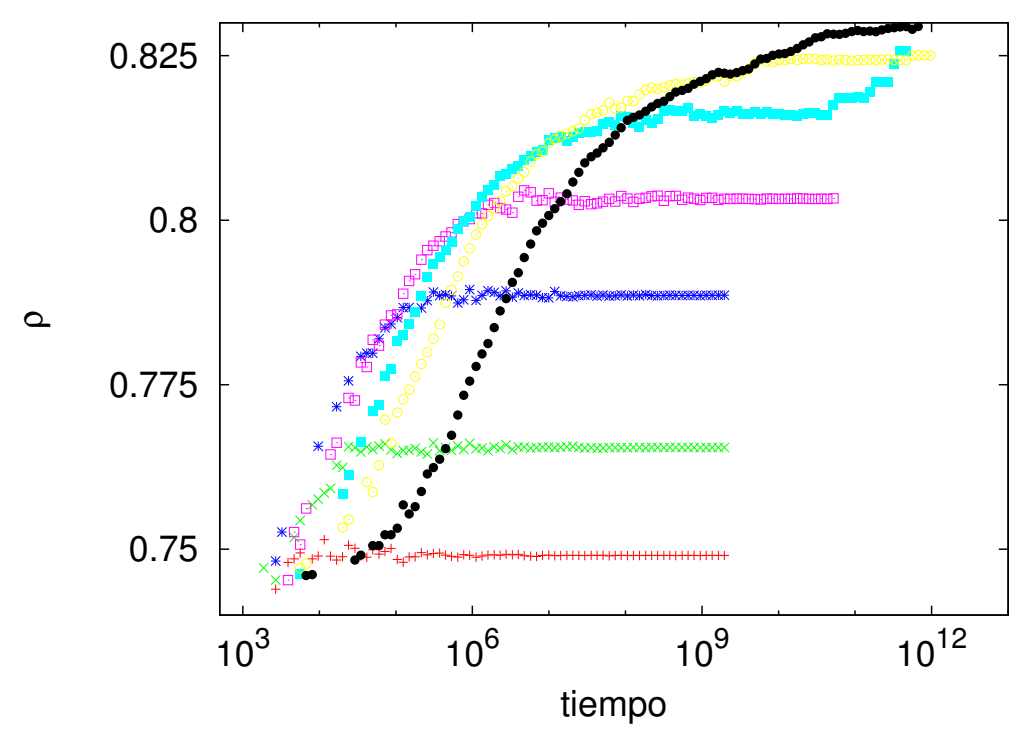

Figura 4.5: Densidad $\rho$ en función del tiempo (de abajo hacia arriba) para: $\mathrm{T} / \mu=$ $\frac{1}{5}, \frac{1}{6}, \frac{1}{7}, \frac{1}{8}, \frac{1}{9}, \frac{1}{10}$ y $\frac{1}{12}$.

\subsection{Límites de la Metaestabilidad}

Intentaremos establecer las temperaturas más bajas a las que el líquido sobreenfriado puede ser equilibrado, es decir, el límite de metaestabilidad. Hemos realizado templados ${ }^{4}$ para distintos valores fijos de $\mathrm{T}_{\mathrm{f}}$ (ver Fig. 4.5) comenzando las simulaciones desde un retículo vació. Cuando $T_{f} \geqslant \mu / 8$, el retículo es llenado rápidamente hasta que la densidad alcanza una meseta, cuya altura depende de la temperatura establecida. Este proceso demora del orden de $\left[10^{3}, 10^{5}\right]$ pasos.

La masa cristalina $\mathrm{m}$, como la definimos en 4.1 , crece mas lentamente que la densidad, sin embargo también alcanza una meseta, como muestra la Fig. 4.6.

El régimen de meseta, es un candidato para el equilibrio, específicamente metaequilibrio, para $T<T_{m} \simeq 0.15 \mu$. Esto es, en tanto el comportamiento en la meseta este sujeto a verificaciones de envejecimiento, que realizaremos en la sección 4.6.

Este es un modelo de corto rango, sin embargo, no esperamos que el estado de metaequilibrio dure por siempre. Efectivamente, para $T=\mu / 9$ observamos que tanto $\rho$ como $\mathrm{m}$ abandonan la meseta hacia valores cristalinos. Interpretamos esto como un régimen de crecimiento cristalino, donde uno o mas núcleos cristalinos supercríticos han sido formados y se encuentran creciendo lentamente. El sistema ya no es un liquido y se encuentra actualmente fuera de equilibrio.

Para $T_{f}=\mu / 12$ el comportamiento es diferente: El crecimiento de $\rho$ y $m$ es len-

${ }^{4}$ Del ingles, quenches 


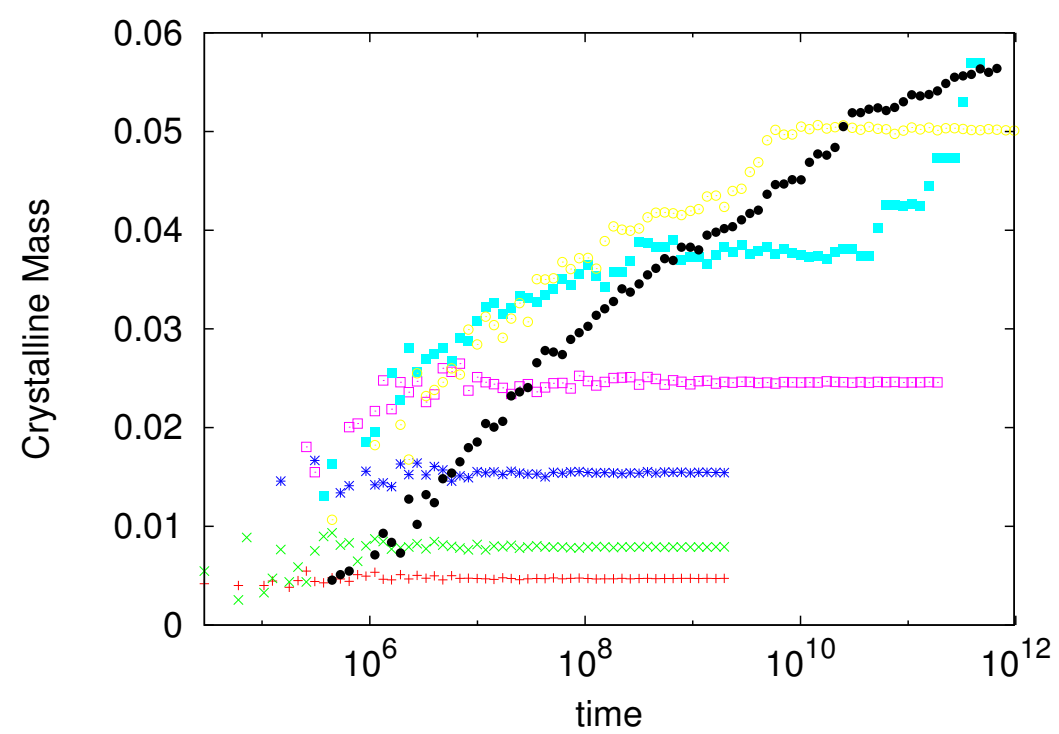

Figura 4.6: Masa cristalina $\mathrm{m}$ en función del tiempo (de abajo hacia arriba) para: $\mathrm{T} / \mu=\frac{1}{5}, \frac{1}{6}, \frac{1}{7}, \frac{1}{8}, \frac{1}{9}, \frac{1}{10}$ y $\frac{1}{12}$.

to, pero el régimen de meseta no es alcanzado nunca. Ambos observables continúan creciendo hacia valores cristalinos, alcanzando valores relativamente altos mas rápidamente que sistemas a valores de $T_{f}$ mayores. El sistema no se encuentra nunca en un estado mestaestable y entra en un régimen de crecimiento de dominios ${ }^{5}$ antes de que el liquido metaestable pueda equilibrar. De esta manera, podemos tomar $\mu / 12 \simeq 0.083 \mu$ como una cota inferior de la temperatura espinodal cinética $T_{s p}$ que introdujimos en la sección 2.4.3. La temperatura estimada de Kauzmann, con un valor de $T_{K} \simeq 0.05 \mu$, se encuentra muy por debajo del limite de metaestabilidad, lo que cuestiona su relevancia.

\subsection{Dinámica de Tiempos Cortos}

En la sección anterior establecimos una cota inferior para la temperatura espinodal cinética $T_{s p}$. En esta sección intentaremos localizar la temperatura espinodal termodinámica $T_{s p}^{*}$ utilizando la técnica de dinámica de tiempos cortos, que mencionamos en 3.5.1.

Notamos que si bien la espinodal termodinámica se encuentra definida únicamente para sistemas con interacciones de rango infinito (campo medio), los trabajo de [Loscar et al., 2009, Loscar et al., 2017] muestran que la dinámica de tiempos cortos permite realizar una determinación de la espinodal termodinámica mediante el estudio de la criticalidad del sistema.

\footnotetext{
${ }^{5}$ Del ingles coarsening
} 
Para ello, utilizaremos la masa cristalina $m(t)$ como parámetro de orden realizando el siguiente procedimiento:

- Preparamos una configuración a una temperatura inicial $\mathrm{T}_{\mathrm{i}}=4 \mathrm{~T}_{\mathrm{m}}$. Esta configuración representa el estado desordenado.

- A tiempo $t=0$ templamos súbitamente el sistema a una temperatura $T_{f}=T_{c}$, donde $T_{c}<T_{m}$. Registramos el parámetro de orden $m$ mientras el sistema relaja durante un tiempo corto de $t \simeq 10^{6}$.

- Buscamos comportamiento de ley de potencia. La temperatura espinodal estará definida como la temperatura donde la ley de potencia tiene una duración máxima.

Puesto que seleccionamos $m(t)$ como parámetro de orden, la cantidad que estamos estudiando sera la fluctuación muestra a muestra, dada por:

$$
\chi_{m}(t)=N \sigma_{m}(t)=N \sqrt{\left\langle[m(t)-\langle m(t)\rangle]^{2}\right\rangle}
$$

Donde $\langle\cdots\rangle$ representa el promedio sobre la historia térmica y sobre la muestra. La fluctuación normalizada $\chi_{m}$ debe ser independiente del tamaño de sistema e invariante ante un desplazamiento de $\mathrm{m}$, lo que resuelve el problema de que $\mathrm{m}$ no es un correcto parámetro de orden para el punto espinodal. De esta manera, en este punto esperamos una dinámica pseudo critica dada por $\chi_{\mathrm{m}} \propto \mathrm{t}^{\phi}$.

La Fig 4.7 muestra la evolución temporal de m, partiendo desde una configuración desordenada a alta temperatura, siendo templada a distintas temperaturas en la zona sobreenfriada. Utilizamos sistemas de tamaño $L=21$ y $L=30$.

Vemos que m es monótonamente creciente, mostrando que es un buen parámetro de orden para detectar el comienzo de formación de la fase sólida. Es importante mencionar que los efectos de tamaño desaparecen luego de $t>10^{2}$ y la técnica comienza a distinguir a las distintas temperaturas para $t>10^{3}$.

La Fig 4.8 muestra $\chi_{m}(t)$ vs $t$ para un sistema de lado $L=30$ para distintas temperaturas. El análisis fue realizado con $10^{4}$ realizaciones de la dinámica. Podemos observar un comportamiento de ley de potencia en el rango de $2 \times 10^{2}<\mathrm{t}<2.7 \times 10^{4}$ (2 décadas) para $\mathrm{T}=\mathrm{T}_{\mathrm{sp}}^{*}=0.104 \mu$. El ajuste de ley de potencia da un exponente $\phi=$ $0.20 \pm 0.01$. En el recuadro de la Fig 4.8 realizamos una comparación de $\chi_{m}(t)$ para un sistema de $L=21$, mostrando nuevamente que los resultados se vuelven independientes de $L$ para $t>200$.

La determinación con esta técnica nos permite estimar la temperatura de la espinodal termodinámica como $\mathrm{T}_{\mathrm{sp}}^{*}=(0.104 \pm 0.004) \mu \simeq 2 \mathrm{~T}_{\mathrm{K}}$. Este resultado, es una segunda 


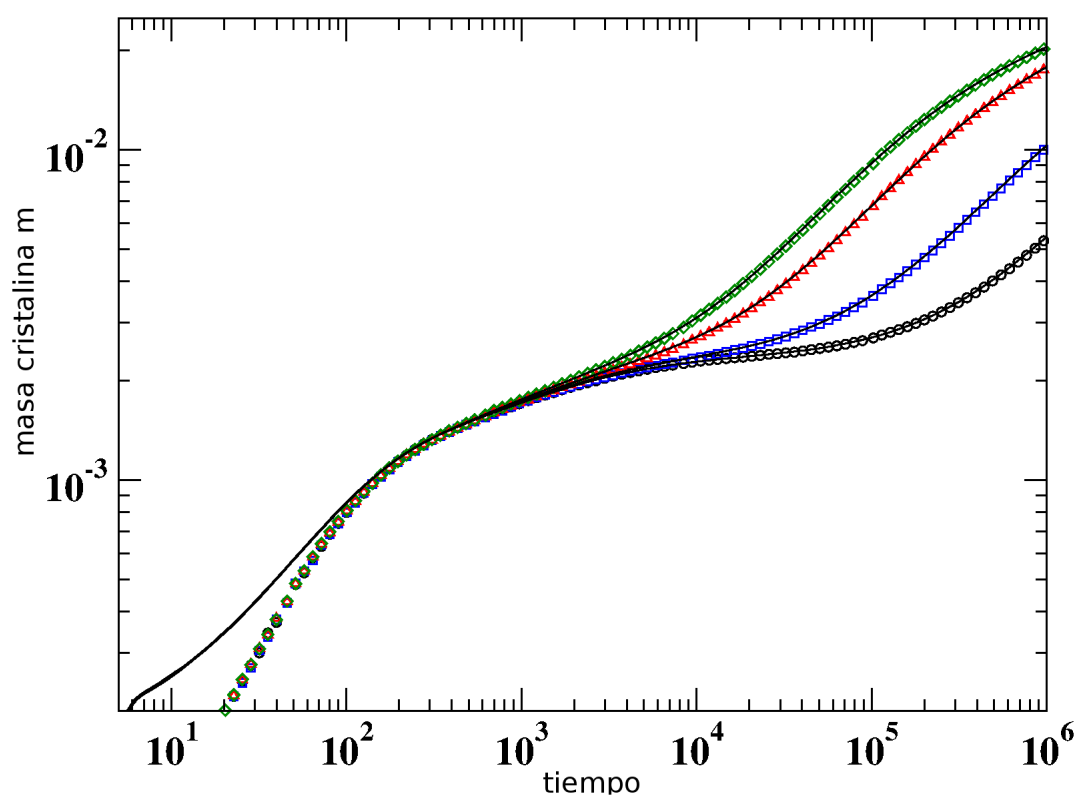

Figura 4.7: Evolución de la masa cristalina $m$ comenzando desde el desorden inicial $\left(\mathrm{T}_{i}=\right.$ $4 \mathrm{~T}_{\mathrm{m}}$ ). Con temperaturas finales $\mathrm{T}_{\mathrm{f}}=0.08 \mu$ (círculos), $0.09 \mu$ (cuadrados), $0.104 \mu$ (triangulo) y $0.112 \mu$ (diamante), utilizando $\mathrm{L}=21$. Las lineas continuas representan las mismas temperaturas, para $\mathrm{L}=30$.

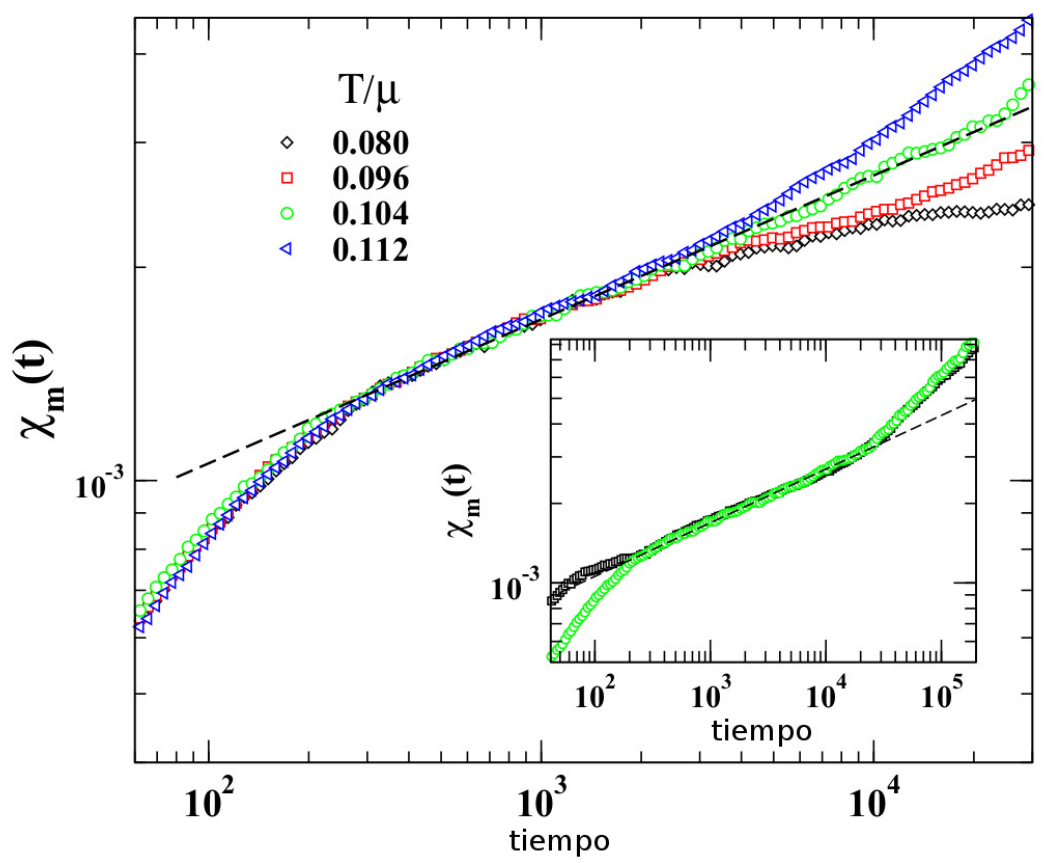

Figura 4.8: La evolución normalizada de las fluctuaciones para distintas temperaturas para un sistema de tamaño $L=30$. Para $T=T_{s p}=0.104 \mu$ obtenemos el máximo comportamiento de ley de potencia. La linea punteada representa un ajuste de ley de potencia, con un exponente $\phi=0.203$. El recuadro muestra resultados para $T=\mathrm{T}_{\mathrm{sp}}^{*}$ 
confirmación de que el punto de Kauzmann es irrelevante para este sistema.

\subsection{Estudios de Envejecimiento}

En las secciones anteriores se realizo un estudio para ver en que regiones existe el líquido. En esta sección estudiamos si dicho líquido es buen formador de vidrios. Ponemos nuestro interés en el estudio del envejecimiento del sistema. Para ello, consideramos el autosolapamiento $\mathrm{Q}\left(\mathrm{t}, \mathrm{t}_{w}\right)$ como una función de 2 tiempos, para tiempos de espera $t_{w} \geqslant 10^{6}$.

En la Fig 4.5, para $T>\mu / 8$ y $t>10^{6}$, el sistema se encuentra en una meseta de densidad . En esta región, observamos invariancia translacional temporal del autosolapamiento, es decir $Q\left(t, t_{w}\right)=Q\left(t-t_{w}\right)$, por lo tanto no se observa envejecimiento.

Para $T=\mu / 9$, encontramos una dependencia de $t_{w}$, como mostramos en la Fig 4.9. En este caso, el sistema exhibe signos de envejecimiento para $10^{6}<t_{w}<10^{8}$. Por sobre este intervalo, las curvas de relajación comienzan a aproximarse las unas a las otras. Esta interrupción del envejecimiento coincide aproximadamente con la aparición de la meseta en $\rho$ y $\mathrm{m}$ (mostradas en Figs. 4.5 y 4.6), y es una indicación de que el liquido esta equilibrando. La meseta correspondiente a $T / \mu$ permanece hasta $t \simeq 10^{11}$, cuando el sistema abandona el equilibrio para comenzar a cristalizar.

Como mencionamos en la sección 2.3.10, en el régimen de envejecimiento, los vidrios estructurales obedecen la siguiente ley de escala:

$$
Q\left(t, t_{w}\right)=f\left(\frac{t}{t_{w}^{v}}\right)
$$

Donde $v$ suele ser cercano a 1 [Kirkpatrick et al., 1989]. En nuestra determinación, vemos que esta relación no vale para los valores de $t_{w}$ presentados en la Fig 4.9, pues las curvas de autosolapamiento coinciden para $t>3 \times 10^{9}$.

Sin embargo, podemos calcular un exponente efectivo de escala $v\left(t_{w}\right)$ [Warren and Rottler, 2013, Strum, 1977]. Definiendo un tiempo característico $t_{c}$ y utilizando un umbral fijo para el autosolapamiento (nosotros elegimos un umbral $\mathrm{Q}\left(\mathrm{t}_{\mathfrak{c}}\right)=0.3$ ), es posible definir un exponente efectivo de escala mediante la siguiente expresión:

$$
v_{e f f}\left(t_{w}\right)=\frac{\partial \ln t_{c}}{\partial \ln t_{w}}
$$

En vez de evaluar numéricamente la derivada presentada en 4.6, realizamos un ajuste sigmoidal para $t_{c}$ vs $t_{w}$ (ver Fig 4.10). Los valores obtenidos de $v$ para $t_{w}=10^{8}$ confirman que el liquido esta alcanzando el equilibrio metaestable. Sin embargo, hallamos 


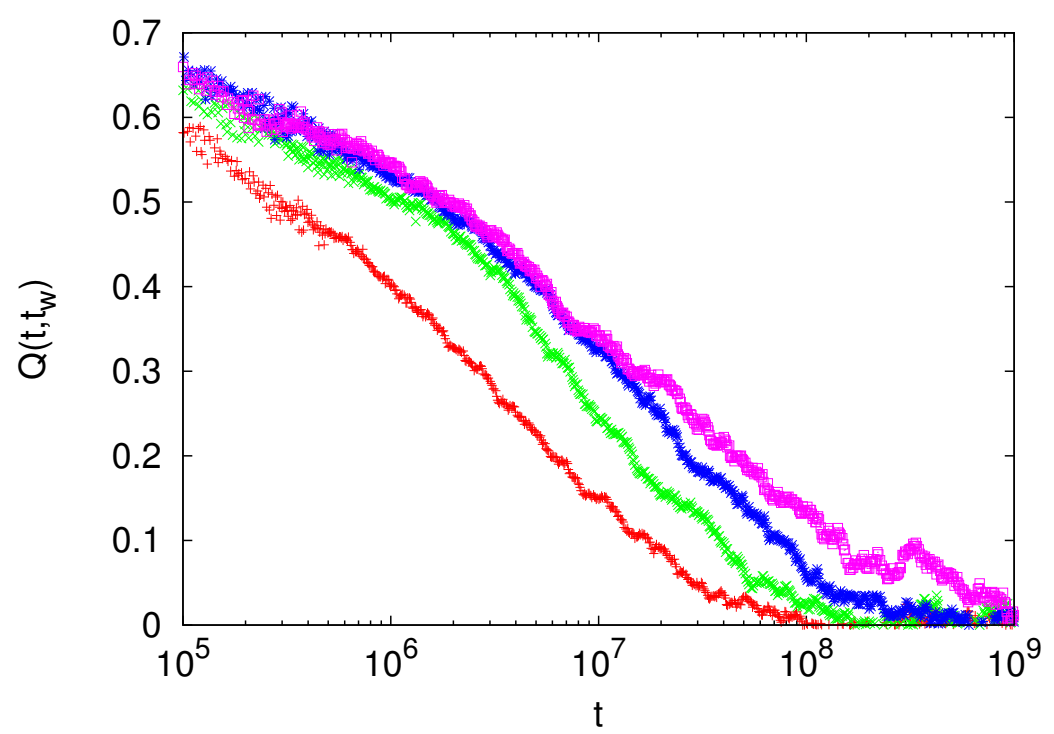

Figura 4.9: Presentamos el autosolapamiento en función del tiempo para $T=\mu / 9$, para tiempos de espera $t_{w}=10^{6}$ (cruces rojas), $t_{w}=10^{7}$ (cruces verdes), $t_{w}=2 \times 10^{8}$ (estrellas azules) y $t_{w}=2 \times 10^{9}$ (cuadrados purpura).

\begin{tabular}{l|c}
\hline $\mathrm{T} / \mu$ & $\gamma_{\max }$ \\
\hline $1 / 8$ & 0.441 \\
$1 / 9$ & 0.476 \\
$1 / 10$ & 0.455 \\
\hline
\end{tabular}

Cuadro 4.1: Estimación de los valores máximos del exponente de envejecimiento $v$ para distintas $\mathrm{T} / \mu$ utilizando un ajuste sigmoidal. Todos los valores hallados se mantienen alejados de $v \simeq 1$.

que $v<0.5$ para todos los casos estudiados, manteniendose muy lejano al valor de $v \simeq 1$ mencionado en [Warren and Rottler, 2013, Kirkpatrick et al., 1989]. Presentamos algunos valores de $v_{\max }$ en la tabla 4.1.

Estos valores de $v$, junto con el comportamiento de $\rho$, nos lleva a interpretar que el modelo se encuentran demasiado cerca del equilibrio como para reproducir el comportamiento vítreo experimental.

En otras palabras, la dinámica se comienza a enlentecer, de manera que demora cada vez mas tiempo en equilibrar el sistema, pero no hay un régimen claro de envejecimiento. El equilibrio es alcanzado relativamente rápido luego de que las cantidades dependientes de 1 tiempo comienzan a estabilizarse.

Como es de esperarse, a temperaturas mas bajas las dinámicas serán mas lentas, de manera que el sistema demorará más en llegar al equilibrio. Sin embargo, como mostramos anteriormente, temperaturas $T<0.1 \mu$ están por debajo del límite de metaesta- 


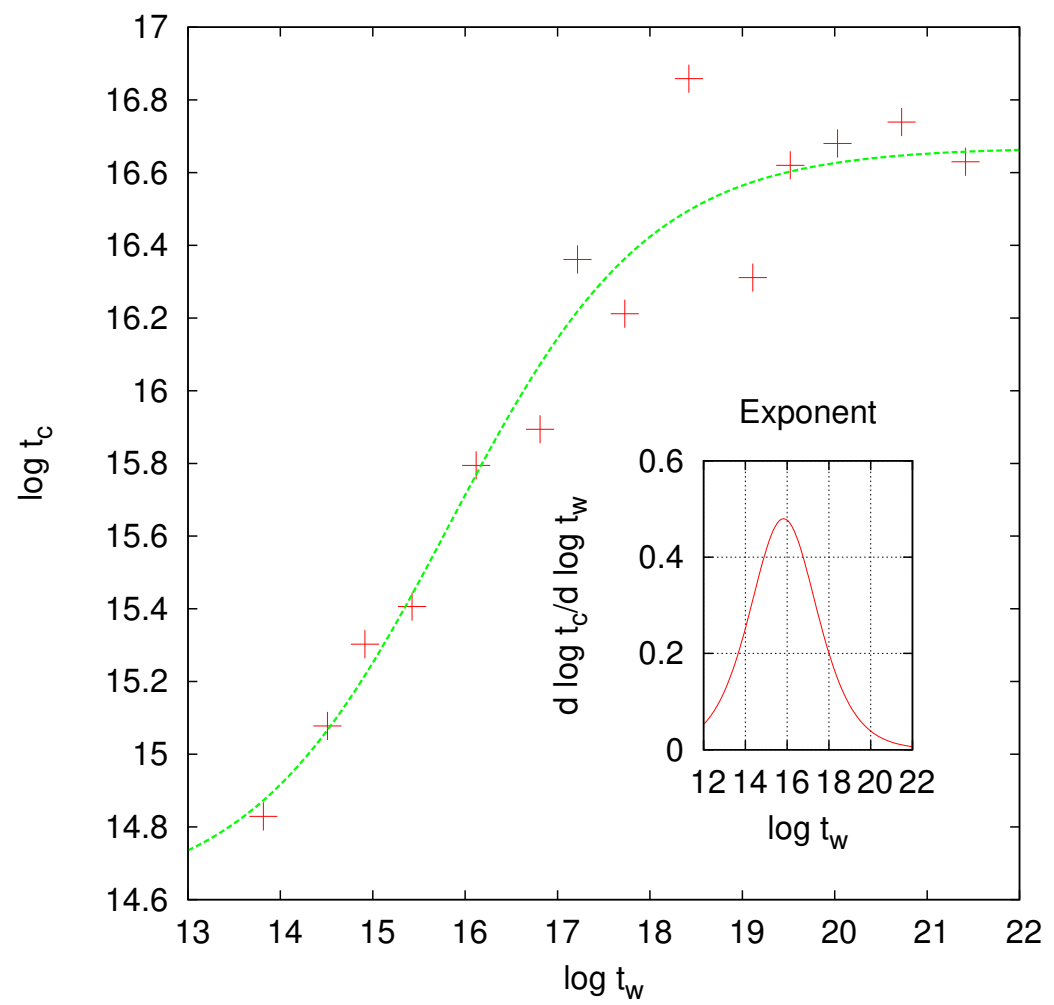

Figura 4.10: Presentamos el tiempo característico de relajación $\left(t_{c}\right)$ vs el tiempo de espera $t_{w}$ para $T=\mu / 9$. La linea representa un ajuste sigmoidal de 4 parámetros, dada por: $S(t)=A+\frac{B}{1+e^{-(t / D-C)}}$. RECUADRO: Determinamos el exponente efectivo mediante $v_{e f f}=\frac{\partial \ln t_{c}}{\partial \ln t_{w}}$ 
bilidad, de manera que el comportamiento fuera de equilibrio de la región corresponde a un régimen de engrosamiento, donde el envejecimiento es cualitativamente distinto al de un vidrio estructural [Berthier et al., 2011]. 


\section{Capítulo 5}

\section{Modelo t154}

Así como en el capítulo 4, analizamos otro modelo de retículo en el cual estudiamos la aparición de la fenomenología vítrea con el aumento de densidad. Este capítulo se organiza de la siguiente manera: En la sección 5.1 presentamos el modelo de retículo de Biroli y Mézard, luego en la sección 5.2 introducimos la variante t154 de dicho modelo. Despues, presentamos un estudio dinámico en la sección 5.3, seguido de un estudio de tamaño finito (ver sección 5.3.1). Por último, en la sección 5.4 presentamos un estudio de la estructura y el comportamiento estático ${ }^{1}$.

\section{1. $\quad$ El Modelo de Biroli Mezard}

Basándose en frustración geométrica, Biroli y Mézard proponen [Biroli and Mézard, 2001] un modelo tridimensional de retículo para el estudio de la transición vítrea. En su trabajo, muestran la existencia de una transición vítrea dinámica similar a las observadas en otros formadores de vidrios, como mezclas binarias Lennard-Jones [Barrat and Latz, 1990].

El modelo de Biroli y Mézard presenta una transición vítrea dinámica y es presentado como una alternativa discreta a los sistemas continuos. Además de un estudio numérico, los autores realizan un estudio de campo medio sobre el retículo de Bethe. Allí, hallan una solución que presenta el mismo escenario que el que se encuentra para campo medio en vidrios de spin [Biroli and Mézard, 2001]. Así como el PCTCC (ver sección 4.1), el modelo de Biroli y Mézard se inspira en la idea de que cada sitio del retículo, representa conjuntos en el continuo de esferas duras de distinta densidad. De esta manera, las partículas en los sitios representan distintas densidades locales en el sistema.

Como muestra la figura 5.1, el modelo consiste en un retículo tridimensional cúbico

\footnotetext{
${ }^{1}$ Algunos resultados desactualizados de este capitulo fueron presentados en [Seif and Grigera, 2016].
} 
de sitios, donde pueden colocarse 0 o 1 partícula. Existen $\ell$ tipos de partícula, donde el tipo representa una restricción de densidad en la siguiente manera: Partículas de tipo $\ell$ admiten hasta $\ell$ primeros vecinos. Esto resulta en que partículas con un tipo $\ell_{i}$ pueden empaquetarse mas que partículas $\ell_{j}$ si vale que $\ell_{i}>\ell_{j}$.

De esta manera, un sitio en el retículo caracteriza la densidad de esferas en una celda local del espacio. La presencia de una partícula en una celda del retículo, representa un arreglo local de esferas muy denso. La ausencia, representa un arreglo local de esferas poco denso. Esto puede producir una frustración geométrica, en el sentido de que quizás no exista un patrón geométrico que pueda ser repetido cubriendo el sistema sin dejar huecos ni solapamientos.

Este modelo forma parte de los LGM que describimos en la sección 2.7. El modelo está definido termodinámicamente, de manera que configuraciones que violen la restricción de densidad están prohibidas. Esto permite hacer un análisis de configuraciones que resulta independiente de la dinámica local, por ejemplo si las partículas recorren el retículo con un numero total constante (ensamble canónico) o si el sistema intercambia partículas con un reservorio (ensamble gran canónico). Adicionalmente, es posible realizar estudios termodinámicos, relacionarlos con observaciones dinámicas y así estudiar la problemática de una transición vítrea ideal.

En [Biroli and Mézard, 2001], los autores estudian un sistema que utiliza una mezcla binaria de partículas, con la siguiente proporción: $30 \%$ tipo 1 y $70 \%$ tipo 2 . Estudiando la densidad del sistema para cambios en el potencial químico $\mu$, de $\dot{\mu} \simeq 10^{-5}$, el sistema cristaliza, produciendo un súbito salto en la densidad. Esto supone un problema al estudiar una dinámica vítrea, dado que la cristalización interviene antes de que el sobreenfriamiento se vuelva significativo. 


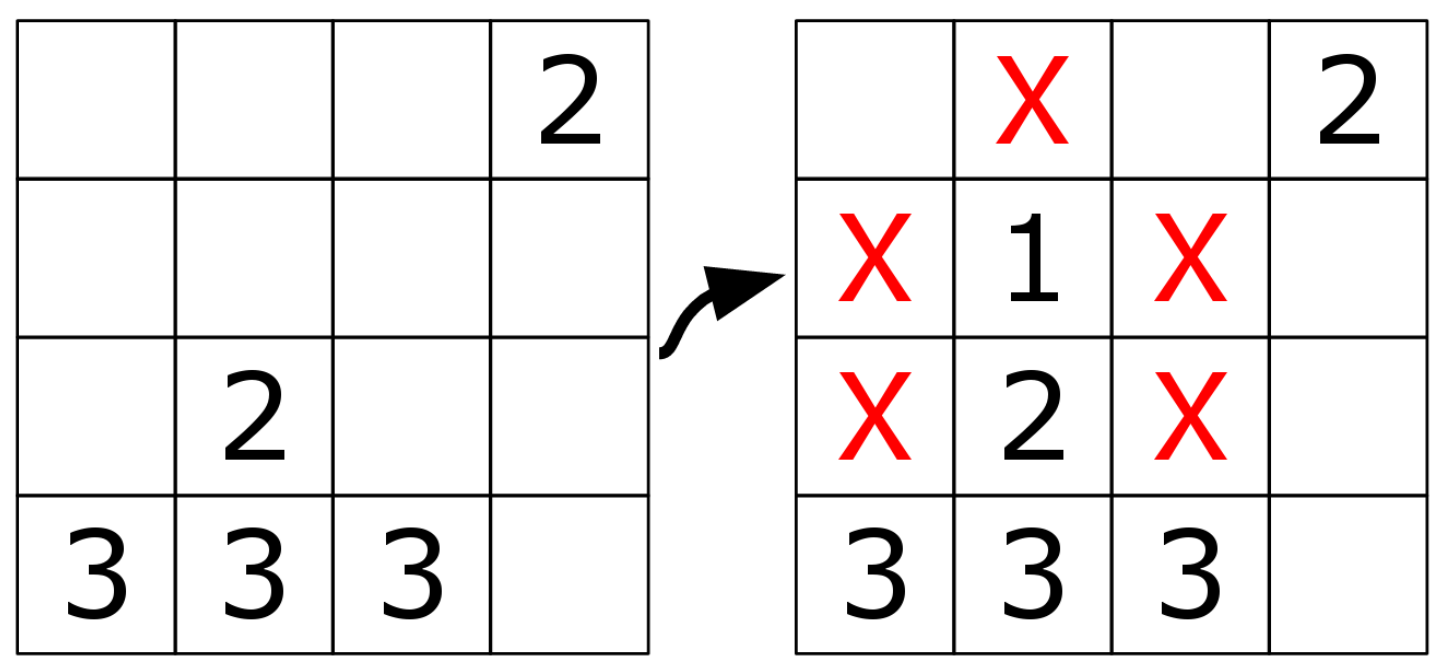

Figura 5.1: Caricatura del modelo de Biroli Mézard para un caso bidimensional lejos de los bordes de la caja. A la izquierda, todos los sitios son capaces de recibir una nueva partícula. En la derecha, agregamos una partícula de tipo 1. Esto desencadena que varios sitios no pueden alojar nuevas partículas (marcados con X).

\section{2. t154: Una Proporción que no Cristaliza}

En los estudios realizados por [Darst et al., 2010], la cristalización era persistente para las mezclas binarias estudiadas. Sin embargo, Darst y colaboradores encontraron una proporción de tres especies de partículas que resulta estable frente a la cristalización para densidades lo suficientemente altas como para estudiar dinámica vítrea. La proporción es la que da nombre al modelo: t154. Esto representa, una mezcla de $10 \%$ de partículas tipo $1,50 \%$ de partículas tipo 2 y $40 \%$ de partículas tipo 3 .

En su trabajo, Darst y colaboradores presentan al t154 en el marco de los modelos de vidrio de retículo (LGM), en contraposición a los modelos constreñidos cinéticamente (KCM). Presentan al t154 como un modelo no propenso a cristalizar, con las propiedades de un liquido formador de vidrio frágil (ver sección 2.3). Detectan movimiento localmente anisotrópico de partículas en escalas temporales intermedias, a pesar de que las reglas que gobiernan al sistema son isotrópicas. Además, presentan la violación de la relación de Stokes-Einstein y el crecimiento de longitudes de escala asociadas a la dinámica heterogénea.

La proporción 154 fue determinada vía prueba y error, buscando tipos de partículas con cristalizaciones en contraposición, frustrando de esta manera la cristalización de la mezcla. Los autores verifican la no cristalización monitoreando cantidades como g(r) y $\mathrm{S}(\mathrm{k})$, así como otros observables del Bulk.

Análogamente al PCTCC, el modelo no cuenta con un hamiltoniano explicito, pues- 
to que o bien los estados están permitidos $(E=0)$ o prohibidos $(E=\infty)$. De la misma manera, estudiamos el sistema tanto en el conjunto canónico (donde solo la densidad $\rho$ será una variable relevante) y en el conjunto gran canónico, donde existen potenciales químicos $\mu_{i}$ con $i=1,2,3$ para cada tipo de partícula.

A diferencia del PCTCC, en este modelo nos encontramos con 2 nuevos desafíos. El primero, es que no conocemos una fase cristalina la cual podemos derretir para determinar la temperatura de fusión y una región de sobreenfriamiento. El segundo, es que nuestro interés está en trabajar con un sistema que mantenga una proporción fija. Esto presenta un problema trabajando en el ensamble gran canónico pues el número de partículas no es constante y esto provoca que la proporción fluctúe.

En efecto cuando trabajamos con el ensamble gran canónico, la cantidad de partículas de cada tipo fluctúa con el tiempo. Nuestro criterio fue mantenernos dentro de una variación menor al $1 \%$ para la proporción. A las configuraciones que respeten esta tolerancia para la regla de proporcionalidad 154 las llamaremos configuraciones legales.

Podemos extraer el conjunto de $\mu_{i}$ para una dada configuración, relevando sus huecos $p_{i}$ y sus partículas $\rho_{i}$. Denotamos huecos, como sitios vacíos que satisfacen las restricciones globales para colocar a una partícula de tipo $\ell$ allí. La formula para calcular $\mu_{i}$ a partir de $p$ y $\rho$ viene dada por:

$$
\beta \mu_{\ell}=\ln \frac{\rho_{\ell}}{p_{\ell}}
$$

Aquí el algoritmo Monte Carlo Cinético nos vuelve a dar una ventaja, pues los huecos son las cantidades naturales que el algoritmo releva para realizar la lista de movidas posibles. Puesto que a priori no conocemos los valores para $\mu_{i}$ que satisfagan la relación 154 deseada, nuestro flujo de trabajo fue el siguiente:

- Crear una configuración legal con un numero de partículas N, con una densidad deseada artificialmente (interrumpiendo la simulación cuando se alcanza el valor deseado de $\rho)$.

- Realizar una simulación canónica partiendo de dicha configuración legal, relevando los huecos y cantidad de partículas en el tiempo.

- Utilizando la expresión (5.1) calcular los $\mu_{\mathrm{i}}$ a partir de la simulación canónica.

- Realizar una simulación gran canónica con el conjunto $\mu_{i}$ obtenido.

- Luego de que el sistema relaje (autosolapamiento), tomar una configuración legal durante una simulación gran canónica. 
- Utilizar dicha configuración legal relajada, para realizar una simulación canónica y así re-calcular $\mu_{i}$.

- Repetir el proceso, hasta que la variabilidad de los números de partícula se mantiene dentro de la proporción 154 .

Nuestro objetivo fue el de establecer una conexión entre la estructura espacial y el comportamiento dinámico. Realizamos medidas espacio-temporales de correlación de la densidad, mediante el observable de autosolapamiento $\mathrm{Q}(\mathrm{t})$ que utilizamos en el modelo anterior (ver capítulo 4). Siendo n la ocupación del sitio (valor 0 o 1), Q(t) viene dado por:

$$
\mathrm{Q}(\mathrm{t})=\frac{1}{\mathrm{~V}} \sum_{\mathrm{i}}^{\mathrm{N}}\left\langle\mathrm{n}_{\mathrm{i}}(\mathrm{t}) \mathrm{n}_{\mathrm{i}}(0)\right\rangle,
$$

\subsection{Dinámica}

Nuestro objetivo fue el de establecer posibles conexiones entre la estructura espacial y el comportamiento dinámico. La principal herramienta utilizada fue el autosolapamiento $\mathrm{Q}(\mathrm{t})$ (ecuación 5.2), el cual encapsula correlaciones espaciales y temporales de la densidad $\rho$.

Consideramos primero un tiempo de relajación $\tau$ medido desde el autosolapamiento $\mathrm{Q}(\mathrm{t})$ para dinámicas canónicas (canónica $(\mathrm{C})$ ) y gran canónicas (gran canónica $(\mathrm{GC})$ ). Ajustamos con una exponencial estirada (relajación Kolrausch-William-Watts [Ediger et al., 1996, Lindsey and Patterson, 1980]) el tiempo de decaimiento del autosolapamiento local Q usando:

$$
\mathrm{Q}(\mathrm{t})=A \exp \left[-\left(\mathrm{t} / \tau_{\beta}\right)^{\beta}\right]+\mathrm{Q}_{\infty}
$$

donde $\mathrm{Q}_{\infty}$ esta fijo a su valor decorrelacionado conocido $\rho^{2}$. El ajuste de los parámetros $\beta$ y $\tau_{\beta}$ determinan la forma y escala temporal, respectivamente, de la relajación. La exponencial estirada puede ser interpretada como la superposición de muchos procesos exponenciales con una distribución ancha de tiempos de relajación exponenciales [Lindsey and Patterson, 1980]. El valor de $\beta$ disminuye con el aumento de la densidad, indicando un mayor estiramiento, ó equivalentemente, un ensanchamiento de la distribución de tiempo de relajación exponencial (Recuadro de la Figura 5.2)

Comparar el $\tau_{\beta}$ de sistemas con distintas formas (distintos $\beta$ ), lleva a confusión. Es por ello que el tiempo de relajación $\tau$ que presentamos, es el promedio sobre la distribución de tiempos de relajación exponenciales que corresponden a la relajación 


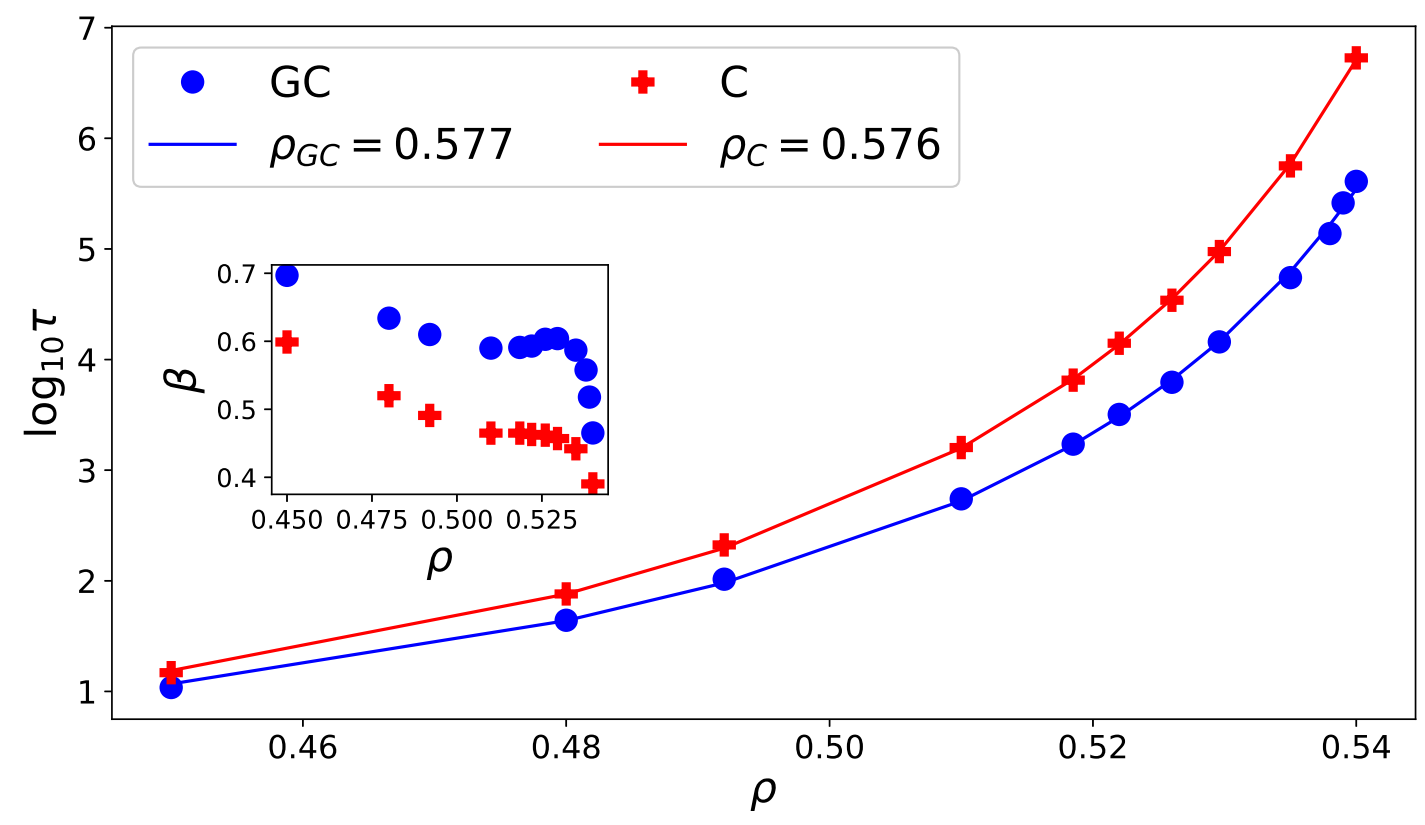

Figura 5.2: Ajuste VFT para dinámicas $\mathrm{C} / \mathrm{GC}$, sistema de tamaño $\mathrm{L}=30$. Notar que la relajación esta presentada en su valor $\tau$ corregido por $\beta$. RECUADRO: Se muestra el coeficiente $\beta$ usado para el ajuste de $Q$ mediante una exponencial estirada.

exponencial estirada [Lindsey and Patterson, 1980]:

$$
\tau=\frac{\tau_{\beta}}{\beta} \Gamma\left(\frac{1}{\beta}\right)
$$

La curva de Arrhenius generalizada [Berthier and Witten, 2009] para los tiempos de relajación, reveló el carácter frágil del modelo (Figura 5.2). La curva puede ser ajustada por una función generalizada de Vogel-Fulchner-Tamman $\tau=\tau_{0} \exp \left[A /\left(\rho-\rho_{K}\right)\right]$.

Debido a las restricciones duras, la dinámica en altas densidades enlentece su relajación. Notar que el precio energético es infinito para configuraciones no permitidas. Por esto, el sistema debe encontrar un camino a la relajación a través de configuraciones permitidas. Podemos imaginar un paisaje de energía que para densidades altas el sistema siente las restricciones energéticas en forma de barreras. A medida que la densidad o el confinamiento aumentan, las configuraciones permitidas se vuelven cada vez menos numerosas, pues las restricciones se vuelven mas difíciles de satisfacer. A densidades aún mayores, en el ensamble canónico, grupos de configuraciones pueden volverse completamente desconectadas (es decir, separadas por barreras de energía infinita) y el sistema se vuelve no-ergódico. La dinámica (no-física) del ensamble GC permite la destrucción y creación de partículas en ubicaciones arbitrarias. Esto disminuye las barreras energéticas, añadiendo conexiones entre configuraciones. En particular, 
la perdida de ergodicidad se evita, pues en el peor de los casos, dos configuraciones pueden ser unidas en un camino que destruye todas las partículas y luego las crea en ubicaciones requeridas.

Así, se pueden esperar tiempos de relajación mas cortos con respecto a la dinámica canónica, al menos para densidades donde esta última se enlentece debido a movidas que llevan a configuraciones prohibidas. Esta expectativa fue verificada, pues la diferencia entre los tiempos de relajación para C y GC mostró un incremento a al aumentar $\rho$. Esto representa una indicación de que la influencia de la estructura en la dinámica aumenta con la densidad, y que densidades mayores a $\rho \simeq 0.45$ pueden ser consideradas influenciadas por el paisaje de energía. Notamos también que $\rho_{K}$, el valor extrapolado de divergencia para $\tau$, es muy similar en ambas dinámicas.

El carácter fragil y el decrecimiento del exponente de estiramiento $\beta$ a medida que $\rho$ aumenta, fue notado por Darst y colaboradores en [Darst et al., 2010]. Allí, el estiramiento progresivo esta fuertemente vinculado, de acuerdo a varios indicadores, a una dinámica crecientemente heterogénea. El autosolapamiento ofrece una manera rápida de verificar este resultado a través de la susceptibilidad dinámica:

$$
\chi_{\mathrm{d}}(\mathrm{t})=\frac{1}{\mathrm{~L}^{3}}\left\langle\mathrm{Q}^{2}(\mathrm{t})\right\rangle-\langle\mathrm{Q}(\mathrm{t})\rangle^{2}
$$

mostrada en la figura 5.3, la susceptibilidad dinámica $\chi_{\mathrm{d}}(\mathrm{t})$ presenta un pico para $\mathrm{t} \approx \tau$, cuya altura crece con la densidad, sugiriendo una escala de longitud dinámica creciente [Darst et al., 2010].

\subsubsection{Efectos de Tamaño Finito}

Como se señalo en el trabajo de [Berthier et al., 2012], el tiempo de relajación de un líquido formador de vidrio debe mostrar efectos de tamaño no triviales en la cercanía de la temperatura de transición vítrea, con efectos más pronunciados esperados para sistemas frágiles. La longitud de correlación a la que los efectos de tamaño finito comienzan a aparecer debe estar relacionada con la longitud de correlación dinámica de una manera que depende del escenario teórico aplicado. Es por esto que investigamos los efectos de tamaño finito en el modelo t154, registrados en el decaimiento del autosolapamiento Q.

Primero consideramos una dinámica canónica con PBCs. Observamos que a medida que el tamaño disminuye, se vuelve más y más probable encontrar muestras atascadas fuera del equilibrio, es decir, cuyo valor $\mathrm{Q}(\mathrm{t})$ nunca alcanza el valor de equilibrio $\mathrm{Q}_{\infty}=$ $\rho^{2}$. En los casos más extremos, el sistema permanece en valores $Q(t) \simeq \rho$, es decir, el 


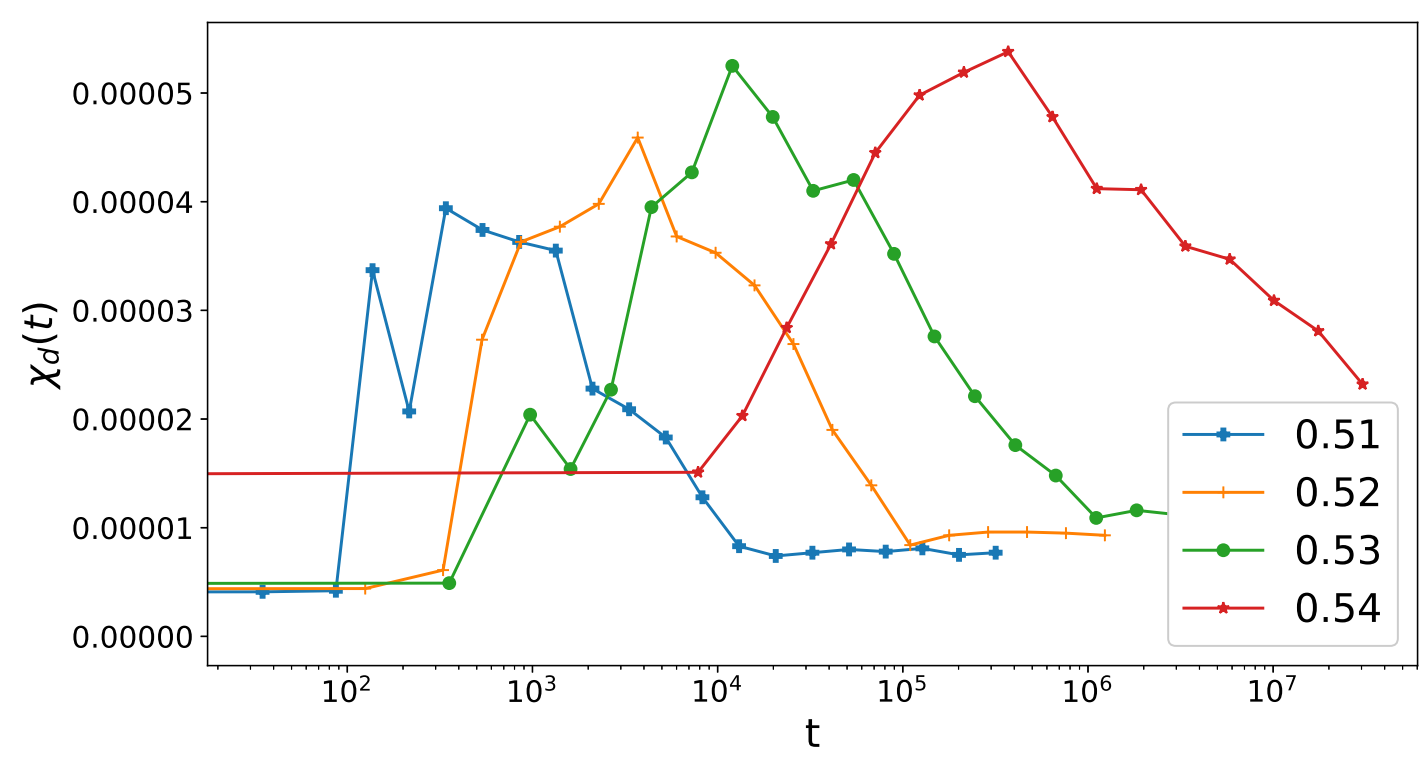

Figura 5.3: Susceptibilidad $\chi_{\mathrm{d}}(\mathrm{t})$ del autosolapamiento para $\mathrm{L}=30$ en el ensamble canónico. La altura y el corrimiento en los picos, a medida que $\rho$ aumenta, no solo muestra un enlentecimiento en la dinámica, sino que tambien exhibe el incremento en la heterogeneidad dinámica.

valor para $\mathrm{Q}(\mathrm{t}=0)$.

Los resultados canónicos que presentamos, fueron obtenidos al tomar promedios únicamente sobre las realizaciones que no se atascan, siguiendo la lógica de [Berthier et al., 2012]. La idea es que este es un modelo minimo con interacciones de núcleo duro, que artificialmente excluye otros mecanismos de relajación (como saltos activados), los cuales un sistema real podría utilizar para relajar. De esta manera, las muestras atascadas contribuyen al promedio con un tiempo de relajación exagerado (infinito). Por eso, excluir del promedio a las configuraciones atascadas da una tendencia cualitativamente más similar al comportamiento de un sistema real. Reportamos tamaños $L \geqslant 10$, pues para $L<10$ los sistemas se atascan muy frecuentemente. Por ejemplo, para $L=6$ a $\rho=0.526$, solo dos de cincuenta realizaciones son capaces de relajar.

La figura 5.4 muestra $\tau$ vs $\rho$ para sistemas PBCs de diferentes tamaños evolucionando con una dinámica canónica. No hubo efectos de tamaño finito visibles hasta para $\mathrm{L}=10$. Repitiendo el análisis para dinámica GC (con mismas condiciones de borde), observamos el surgimiento de ligeros efectos de tamaño para $\rho \gtrsim 0.5$ (ver Figura 5.5, donde ninguna muestra presenta atascamiento). A pesar de que $L=10$ parece ser ligeramente mas lento que otros tamaños, no surge una tendencia clara y el efecto resultante parece ser principalmente una dispersión de los puntos.

Finalmente, consideramos la relajación $q(t)$ con dinámica $\mathrm{GC}$ en cavidades $\mathrm{ABCs}$ 


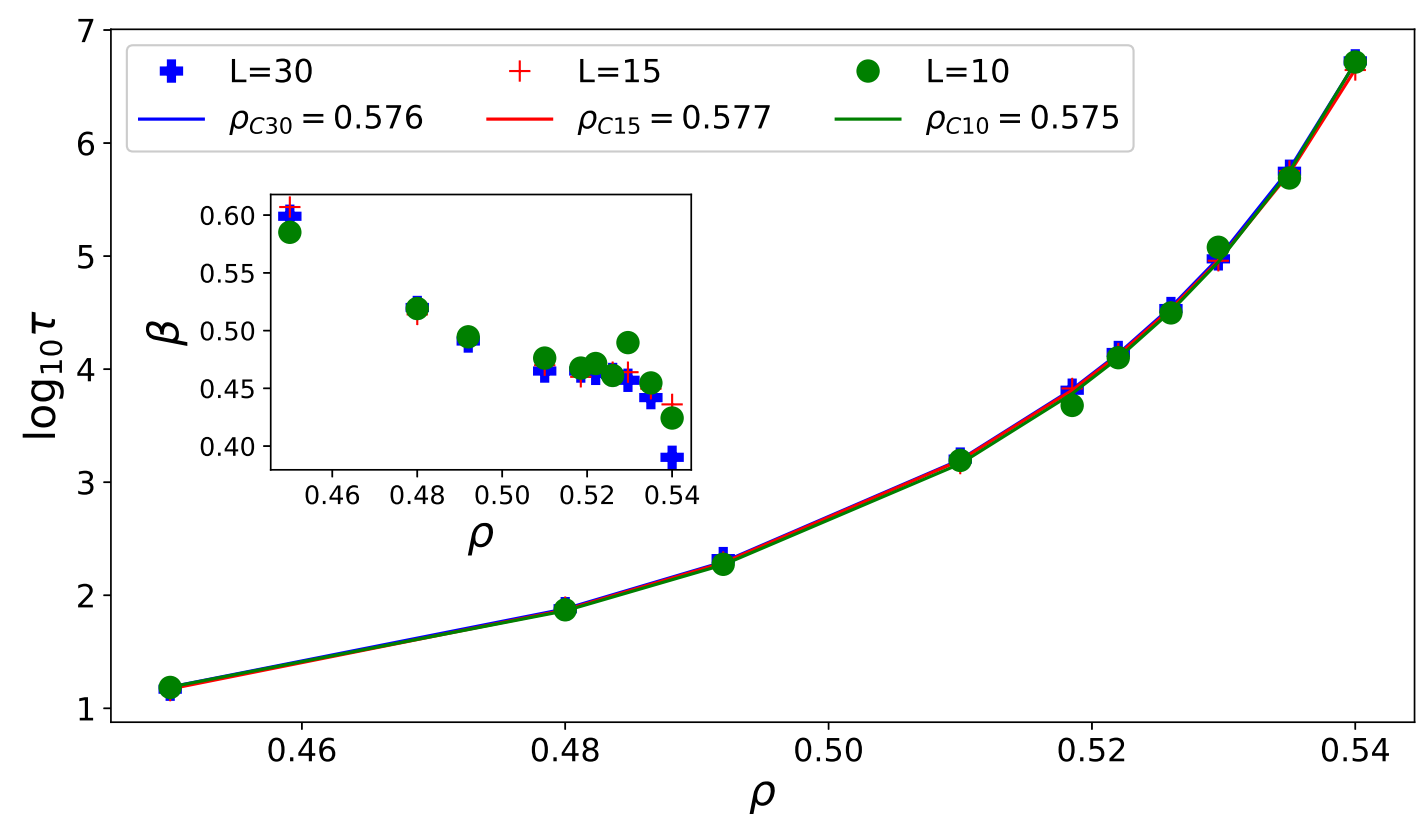

Figura 5.4: Tiempo de relajación $\tau$ vs $\rho$ usando PBCs Canónico, para distintos tamaños de $\mathrm{L}^{3}$. Posee tiempos de relajación mayores al caso GC (ver figura 5.5), pero como sugiere el ajuste VFT, no hay una dependencia visible con L. RECUADRO: Los coeficientes $\beta$ del ajuste de la exponencial estirada, si bien dependen de $\rho$, no muestran una dependencia visible con $\mathrm{L}$

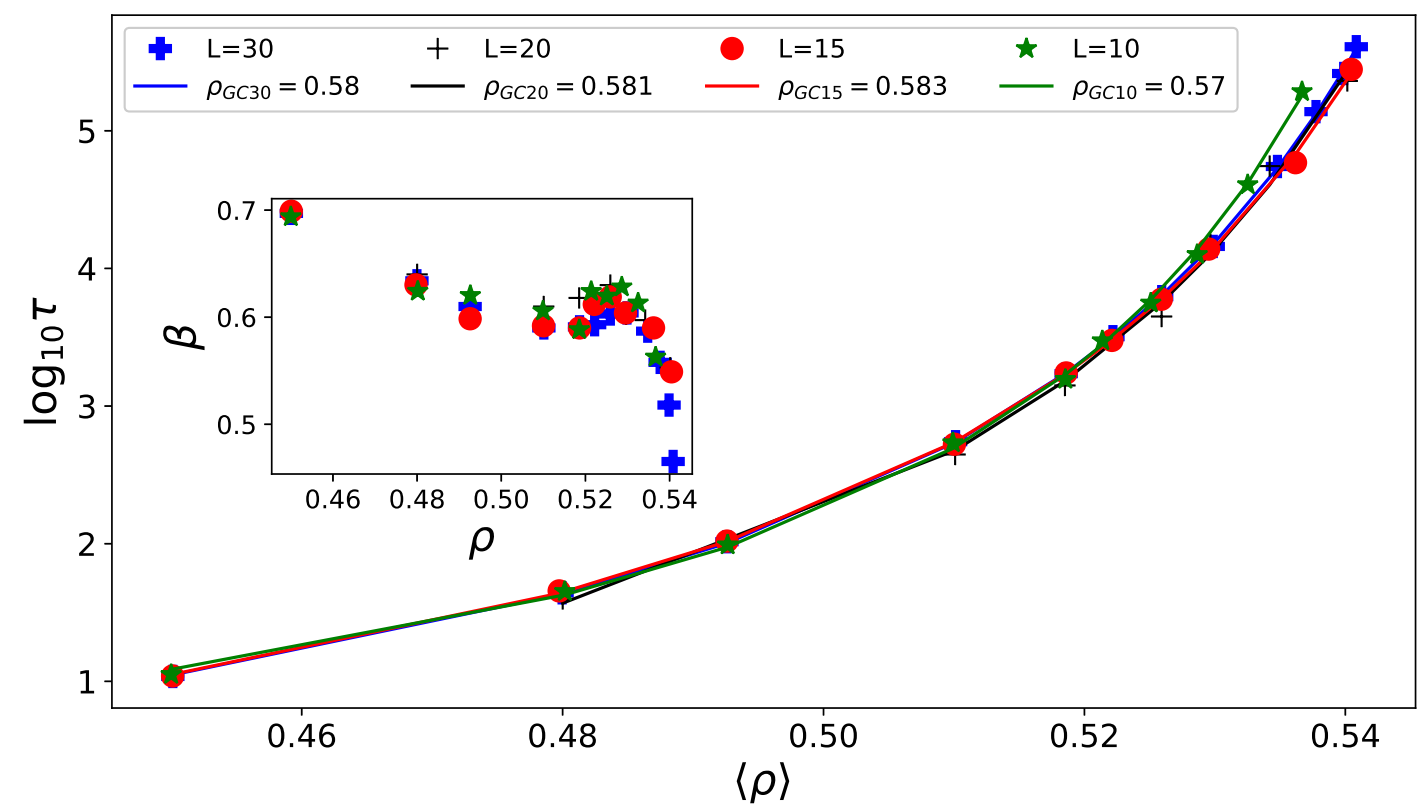

Figura 5.5: Tiempo de relajación $\tau$ vs $\langle\rho\rangle$ usando PBCs GC. Los sistemas grandes parecen relajar ligeramente mas rápido en configuraciones de alta densidad, basándonos en el ajuste VFT. RECUADRO: Coeficientes $\beta$ del ajuste de la exponencial estirada. 


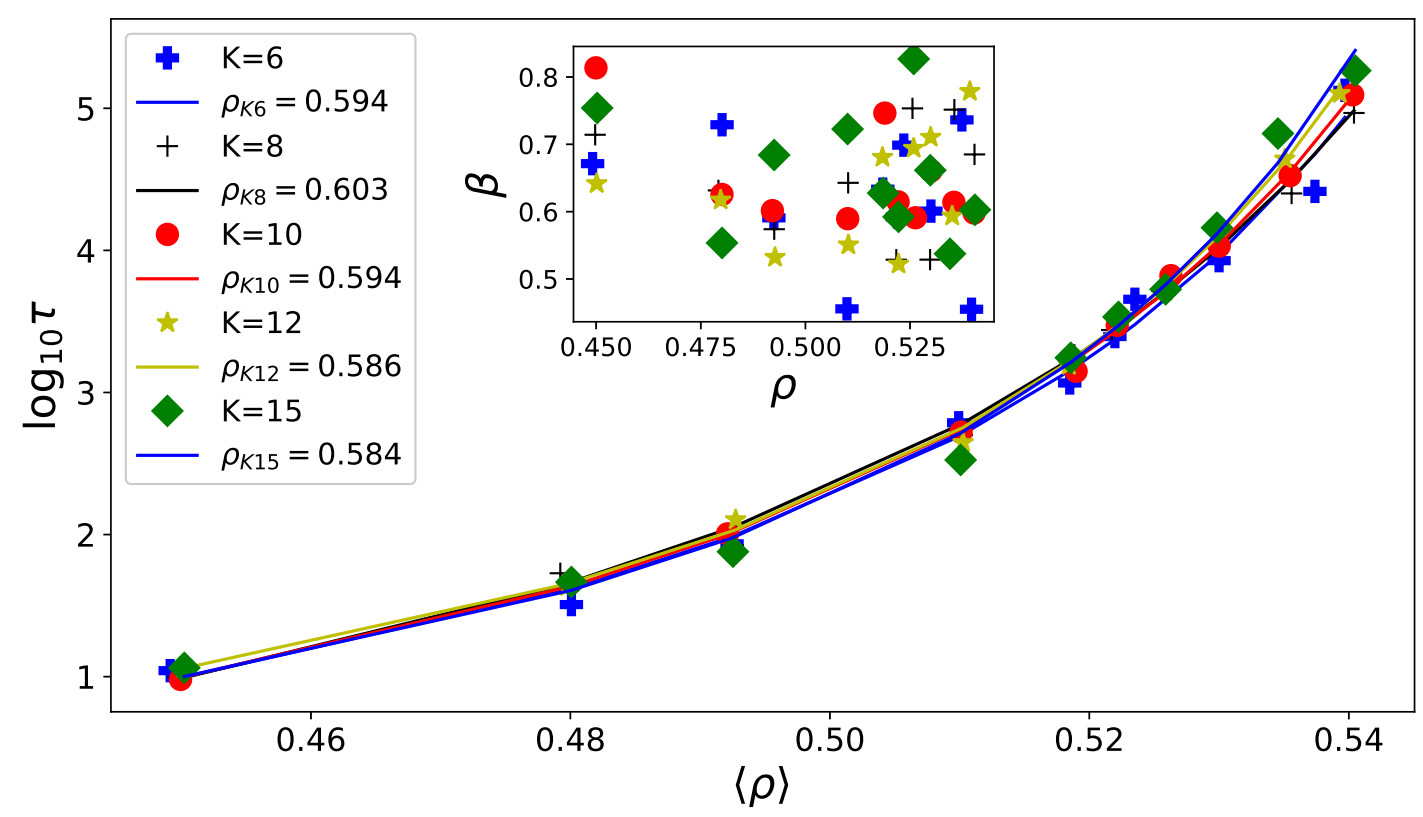

Figura 5.6: Tiempo de relajación PTS $\tau$ vs $\langle\rho\rangle$ usando ABCs GC. El ajuste VFT sugiere que las cavidades mas pequeñas, relajan mas rápidamente. RECUADRO: Coeficientes $\beta$ de la exponencial estirada. A diferencia del caso PBC, la variabilidad de $\beta$ aumenta con $\rho$.

(las mismas cavidades utilizadas para obtener la longitud de correlación estática en la sección 5.4). Los efectos de tamaño, exhibidos en la figura 5.6, son observados para $\rho \gtrsim$ 0.5. Por sobre esta densidad, existe un esparcimiento de puntos sobre las curvas, donde las cavidades pequeñas parecen relajar mas rápidamente que las grandes. Aclaramos que no presentamos un estudio de sistemas confinados por cavidades ABCs para dinámica canónica, pues en estos casos el tiempo de relajación crece muy rápidamente y la mayoría de las muestras resultan completamente atascadas, incluso para las cavidades de mayor tamaño.

\subsection{Estructura}

En esta sección examinamos el comportamiento de la densidad y sus correlaciones. Primero consideramos la densidad $\rho$, como una función del multiplicador de Lagrange $\alpha_{1}=\beta \mu_{1}$. Donde $\alpha_{1}$ representa el logaritmo de la fugacidad de partículas de clase 1 , exhibido en las figuras 5.8, 5.7. Hallamos que para $\alpha_{1}>4$ (correspondiente a $\rho \gtrsim 0.51$ ) existe una dependencia de tamaño en las curvas. Sin embargo, la dependencia no es monotona en $L$ y los valores resultan coincidentes usando las varianzas como barras de error. 


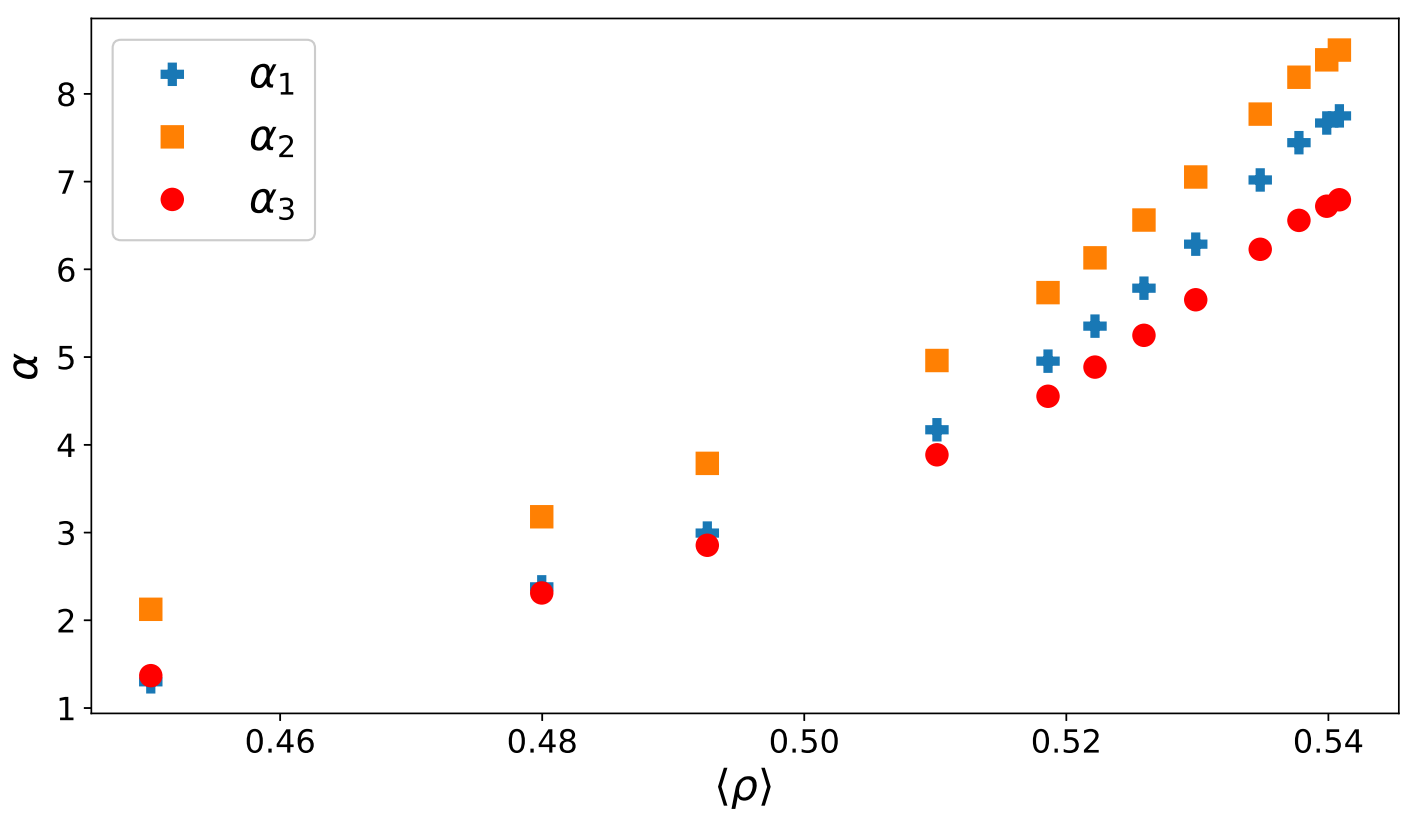

Figura 5.7: Densidad $\rho$ vs $\alpha$ determinados para $L=30$. Observamos que debido a la sensibilidad del sistema a los valores de $\alpha_{l}$, resultó imposible obtener una expresión generalizada. Por ello optamos por determinar cada densidad mediante el proceso descrito en la sección 5.2

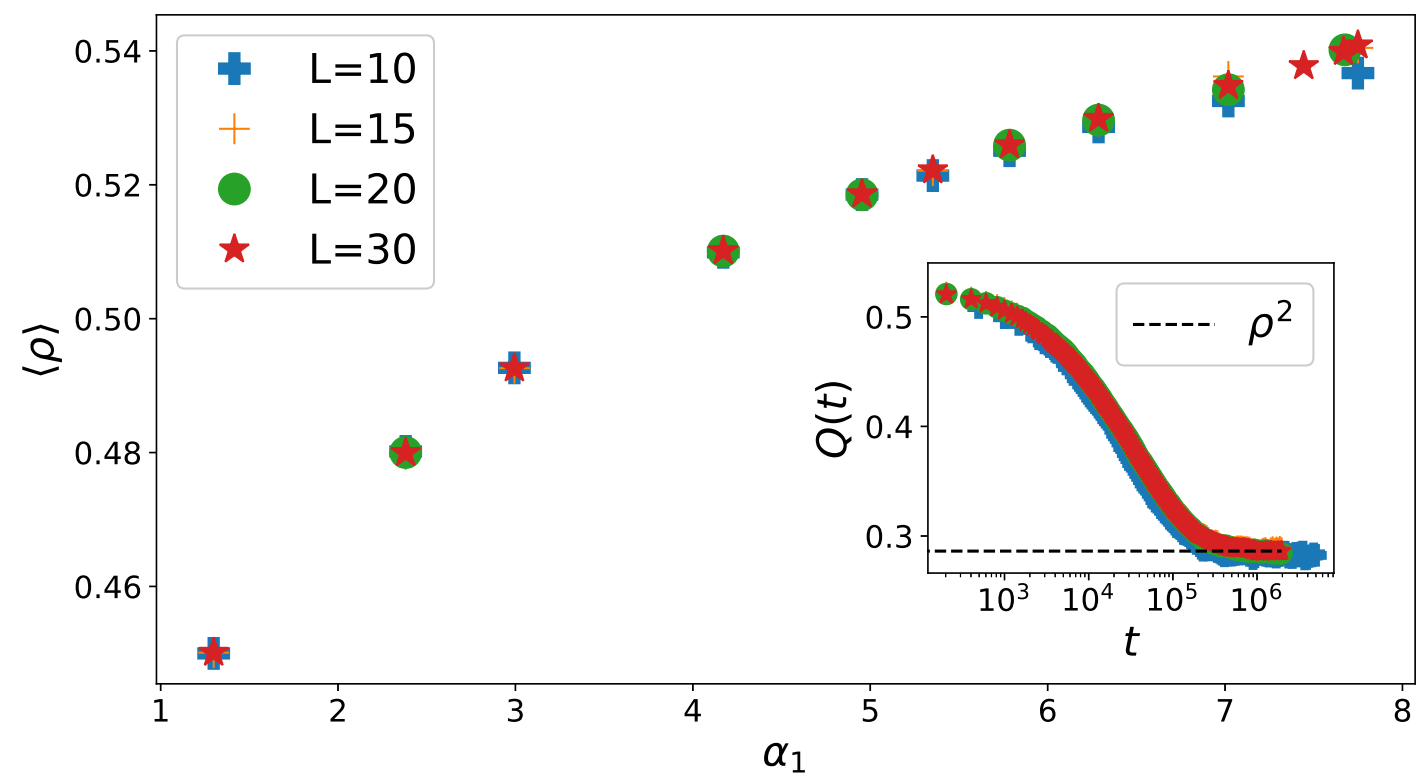

Figura 5.8: Densidad $\rho$ vs $\alpha_{1}$ para dinámica PBCs GC. Los valores mas altos de $\alpha_{l}$ producen $\langle\rho\rangle$ que varia con L. Notamos que el tamaño de los puntos, considera la incerteza de la determinación. RECUADRO: Autosolapamiento $\mathrm{Q}(\mathrm{t})$ vs tiempo para diferentes tamaños de caja L, usando una dinámica PBCs GC (con $\alpha_{l}$ constante. Como esperabamos, $\mathrm{Q}(\mathrm{t})$ para $\mathrm{PBCs} \mathrm{GC}$ decae a $\rho^{2}$ cuando termaliza. 


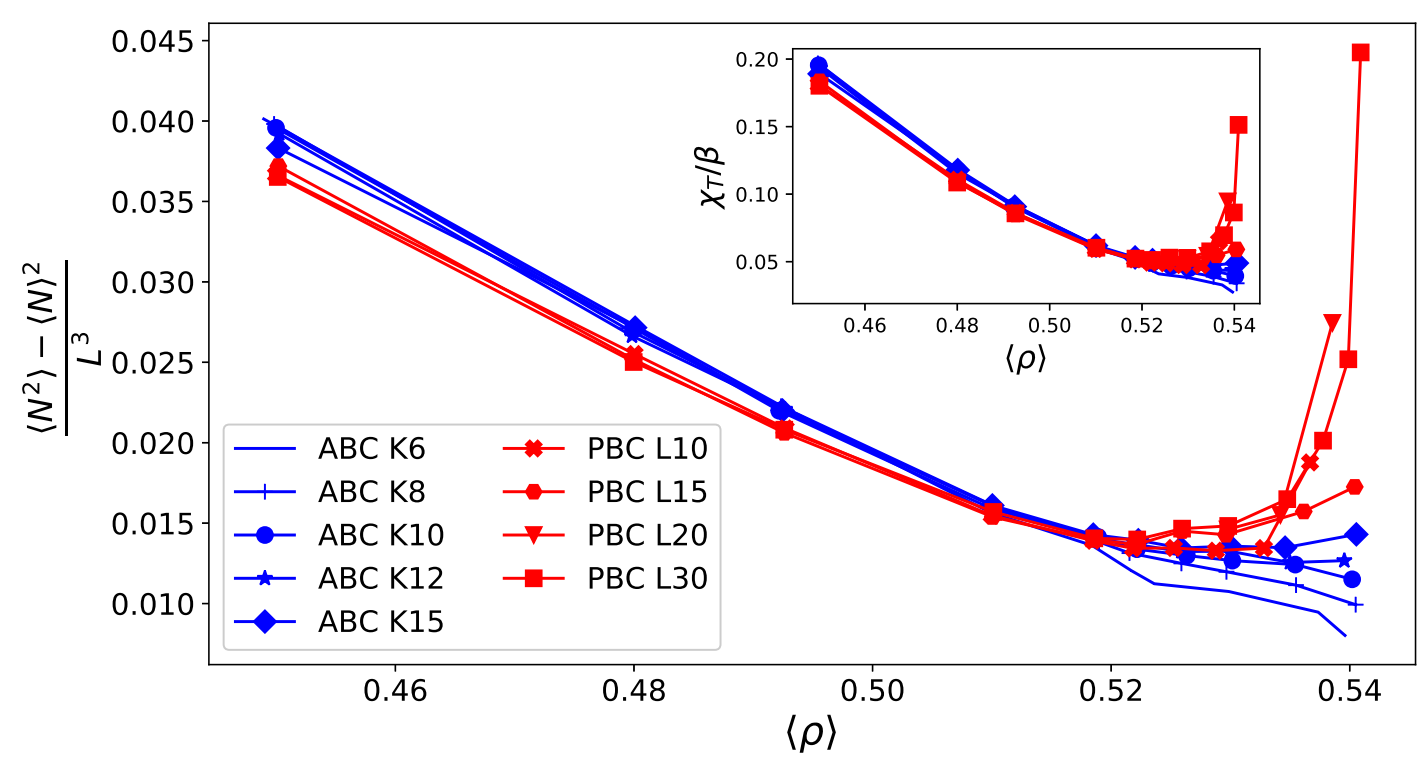

Figura 5.9: Fluctuaciones de la densidad para dinámicas GC en condiciones ABCs y PBCs. El crecimiento de las fluctuaciones sugiere un aumento de la longitud de correlación. RECUADRO: Compresibilidad isotérmica $\chi_{T}$ para los sistemas GC estudiados.

El número global de fluctuaciones en el ensamble GC ayuda a entender las observaciones previas. La varianza del número total de partículas dividas por el volumen, $\left[\left\langle N^{2}\right\rangle-\langle N\rangle^{2}\right] / L^{3}$, esta presentada en la Figura 5.9. En el limite termodinámico, esta cantidad debería ser independiente del tamaño. Sin embargo, como muestra la figura, el régimen es alcanzado solo para sistemas relativamente pequeños cuando la densidad es baja. Cuando $\rho \gtrsim 0.51$, los efectos de tamaño finito se vuelven evidentes para $\mathrm{L} \leqslant 15$. Bajo condiciones PBCs, las fluctuaciones alcanzan un claro mínimo alrededor de $\rho \simeq 0.53$ y luego experimentan un estrepitoso incremento. Este incremento es pequeño o despreciable para ABCs. De esta manera, la aparición de efectos de tamaño finito en el tiempo de relajación para cavidad ABCs, en las curvas $\langle\rho\rangle$ vs. $\alpha_{1}$ y en el número global de fluctuaciones, están correlacionados y esto comienza a ser evidente a partir de $\rho \simeq 0.51$

La fluctuación del número de partículas en el ensamble GC esta relacionada a la compresibilidad isotérmica $\chi_{\mathrm{T}}=-(1 / \mathrm{V})(\partial \mathrm{V} / \partial \mathrm{P})_{\mathrm{T}, \mathrm{N}}$ a través de [Hansen and McDonald, 1990],

$$
\frac{\chi_{\mathrm{T}}}{\beta}=\frac{\left\langle\mathrm{N}^{2}\right\rangle-\langle\mathrm{N}\rangle^{2}}{\rho\langle\mathrm{N}\rangle}
$$

mostrado en la figura 5.9. Las fluctuaciones del número de partículas también están 
relacionadas a la integral de la correlación conectada de densidad,

$$
\begin{gathered}
C_{c}(r)=\langle\rho(0) \rho(r)\rangle-\langle\rho\rangle^{2} \\
\frac{\left\langle N^{2}\right\rangle-\langle N\rangle^{2}}{L^{3}}=\int d^{d} r C_{c}(r),
\end{gathered}
$$

De manera que el fuerte incremento en el número de fluctuaciones, implica que la correlación estática densidad-densidad están aumentando, sugiriendo que una longitud de correlación esta creciendo. Sin embargo, es importante notar que el valor de la integral (o equivalentemente de las fluctuaciones) es muy similar entre las densidades mas bajas y las mas altas. También, los prefactores dependientes de la densidad, hacen que la interpretación de este incremento en fluctuationes sea difícil. De manera que para medir una longitud de correlación directamente, nos volcamos al uso de la correlación punto-conjunto PTS [Montanari and Semerjian, 2006b]. Como mencionamos en la sección 2.5, PTS es una medida agnóstica del orden, en el sentido en que no asume nada sobre el parámetro de orden, o de que tipo de orden se forma. Además, posee la ventaja de que es capaz de detectar la presencia de orden, aun en ausencia de valores apreciables de correlaciones de dos puntos.

Para hallar PTS computamos el decaimiento del autosolapamiento (ver Ecuación 5.2) para sistemas a diferentes potenciales químicos y confinados en cavidades cubicas de lado K con ABCs. En este caso, definiremos como punto de la correlación PTS, a un cubo de tamaño $3^{3}$ en el centro de la caja. De esta manera, todos los volúmenes $\mathrm{K}^{3}$ de cavidad utilizados, deben cumplir $\mathrm{K}^{3}>3^{3}$. Tanto el autosolapamiento global $\mathrm{Q}(\mathrm{t})$, es decir el de toda la cavidad con si misma, así como el autosolapamiento $q(t)$ de un cubo de tamaño $3^{3}$ en el centro de la cavidad, fueron computados (ver Figura 5.10). La correlación PTS fue obtenida como el limite $t \rightarrow \infty$ de $q(t)$. Todos los resultados PTS fueron obtenidos en el ensamble GC, donde la dinámica es mas rápida y permite al sistema equilibrar hasta densidades de $\rho=0.54$.

La dinámica en el ensamble canónico es muy lenta y resulta imposible de equilibrar, aún para cavidades moderadamente confinadas en simulaciones canónicas (ver sección 5.3). Sin embargo, la elección de la dinámica es irrelevante para los resultados estructurales, siempre que el sistema halla termalizado. Dado que hemos hallado dependencia del tamaño del sistema con el potencial químico, hemos verificado que la composición de las cavidades se mantenga en la proporción 154. Hemos hallado fluctuaciones en la composición de las cavidades mas pequeñas, así como para sistemas PBC pequeños, pero en ningún caso superior al $3 \%$.

Cuando la estructura se decorrelaciona completamente, así como cuando termaliza 


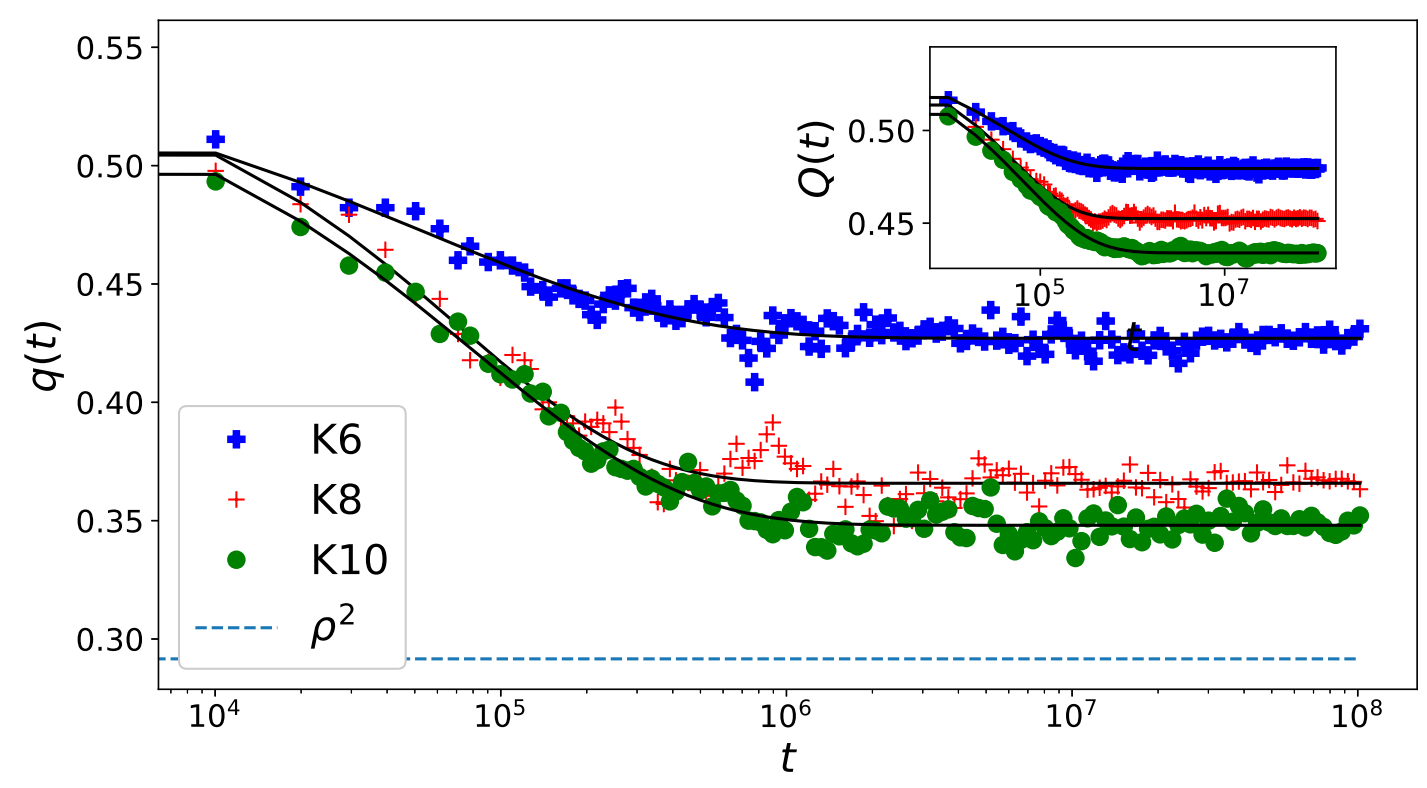

Figura 5.10: Presentamos $q(\mathrm{t})$, y en el RECUADRO: $\mathrm{Q}(\mathrm{t})$, en función del tiempo para $\langle\rho\rangle=0.54$ y cavidades de tamaño $\mathrm{K}^{3}$. En el autosolapamiento $\mathrm{Q}$, todos los valores asintóticos caen por arriba del valor PBCs decorrelacionado (dado por $\rho^{2}$ ), mientras que en el caso punto conjunto q, solo las cavidades mas pequeñas quedan por arriba de el.

en PBCs, alcanza el valor asintótico conocido $\left\langle\rho^{2}\right\rangle$, tanto para $\mathrm{Q}(\mathrm{t})$ como para $\mathrm{q}(\mathrm{t})$ (ver Figura 5.8). La presencia de correlaciones estructurales es revelada por el hecho de que el valor asintótico para la cavidad es mayor que en el caso PBCs. Por supuesto que una simulación que es demasiado corta para termalizar el sistema podría producir un alto valor espureo del autosolapamiento asintótico. Para verificar que el sistema efectivamente ha equilibrado, y que estamos midiendo el verdadero equilibrio PTS, realizaremos una prueba condición inicia $\beta$ (BIC) como la descripta en la sección 3.5.2. Presentamos una de las pruebas realizadas en la Figura 5.11: Observamos que el mismo valor del autosolapamiento es alcanzado comenzando desde configuraciones independientes, tanto con alto como con bajo autosolapamiento inicial. Cuando computamos el valor asintótico de $\mathrm{q}_{\infty} \mathrm{y} \mathrm{Q}_{\infty}$, se promedio sólo para tiempos tales que las dos curvas en las que la prueba BIC coinciden.

Tanto para $\mathrm{Q}(\mathrm{t})$ como para $\mathrm{q}(\mathrm{t})$, resulta claro que para cavidades pequeñas, el borde ejerce una influencia significativa en las partículas en el interior de la cavidad. Usamos los valores $\mathrm{q}_{\infty}(\mathrm{K})$ y $\mathrm{Q}_{\infty}(\mathrm{K})$ para extraer una longitud de correlación. Ambas longitudes contienen información sobre las correlaciones estructurales, pero, si bien Q es menos ruidosa, también resulta mas difícil de interpretar. 


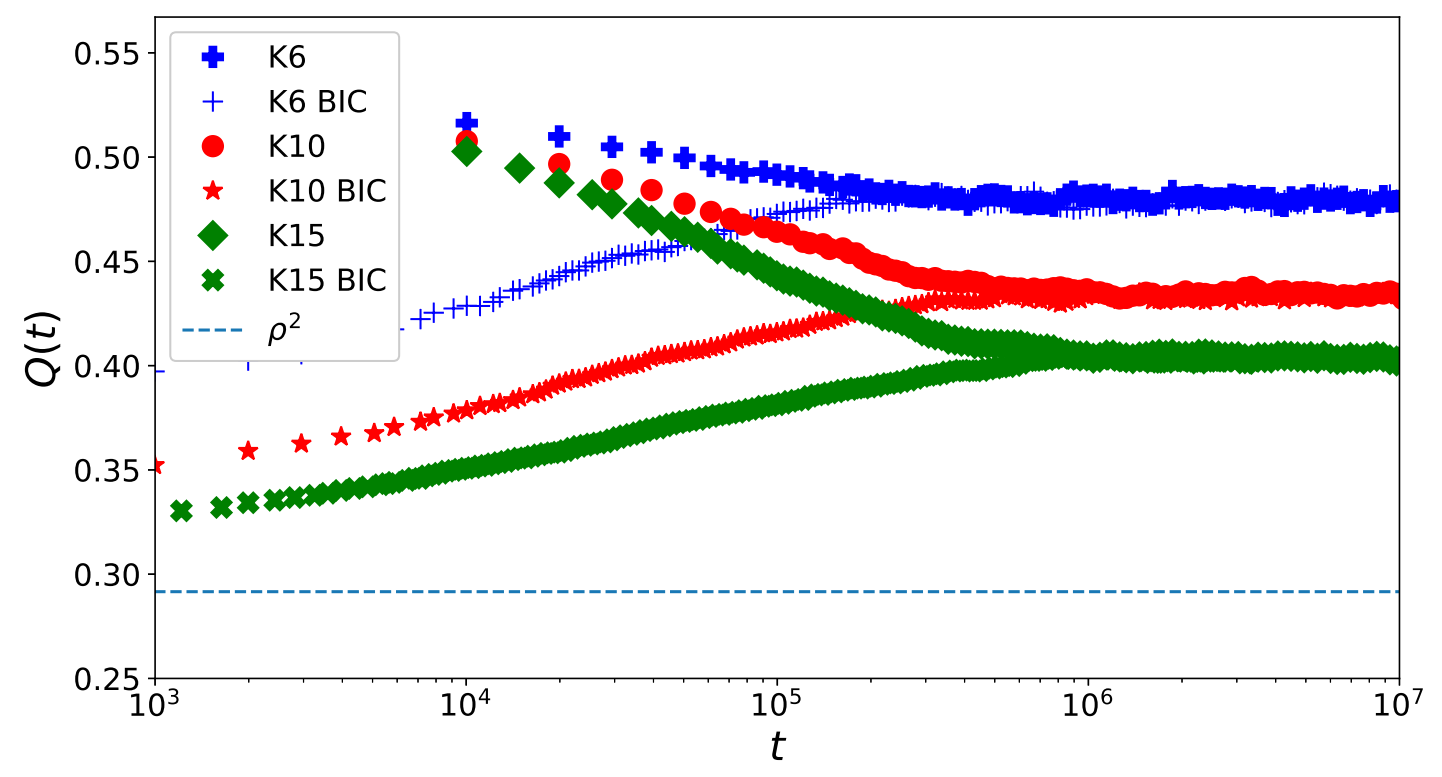

Figura 5.11: Prueba BIC para $\rho=0.54$ para cavidades de tamaño $\mathrm{K}^{3}=6^{3}, 10^{3}, 15^{3}$. Podemos ver que la prueba resulta positiva en los tres casos, dado que ambos puntos de partida alcanzan el mismo valor asintótico. De esta manera, concluimos que las configuraciones han termalizado.

Comenzamos con la correlación PTS para $\mathrm{q}_{\infty}(\mathrm{K})$, presentada en la figura 5.12. Observamos que una simple exponencial ajusta adecuadamente el decamiento de PTS, del cual podemos extraer la longitud de correlación そpTS

$$
\mathrm{q}_{\mathrm{c}}(\mathrm{K})-\mathrm{q}_{\mathrm{o}}=\left(\mathrm{q}_{1}-\mathrm{q}_{\mathrm{o}}\right) \exp \left[-\mathrm{K} / \xi_{\mathrm{PTS}}\right]
$$

Donde $\mathrm{q}_{0}=\langle\rho\rangle^{2}$ y $\mathrm{q}_{1}$ y $\xi_{\mathrm{PTS}}$ son parámetros de ajuste. Cuando $\mathrm{K} \gg \xi_{\mathrm{PTS}}$, el centro de la cavidad es libre para reacomodarse como si estuviese sujeto a condiciones PBCs. De esta manera, $\xi_{\text {PTS }}$ mide cuán lejos la estructura local influencia el arreglo de otras partículas. La figura 5.13 muestra que $\xi_{\text {PTS }}$ aumenta monótonamente, con una pendiente más pronunciada para $\rho>0.53$. El decaimiento de $q_{c}(K)$ está adecuadamente ajustado por una exponencial para todas las densidades. No hallamos presencia de comportamiento no-exponencial, como el descrito por las referencias [Biroli et al., 2008, Hocky et al., 2012] [Gradenigo et al., 2013] referido a la aparición de multiples estados metaestables de la teoría RFOT, presentada en la sección 2.6.4.

La información estructural contenida en $\mathrm{Q}_{\infty}(K)$ está codificada en una manera más complicada: incluso para cavidades mucho más grandes que $\xi_{\mathrm{PTS}}$, donde las distancias involucradas son mayores a las que pueden ser medidas por PTS, el valor de $\mathrm{Q}_{\infty}$ supera al caso PBCs, debido a los efectos de un campo de confinamiento causado por las 
partículas en el borde congelado de la cavidad. Es decir, aún cuando el centro de la cavidad esté completamente decorrelacionado con el borde, el autosolapamiento global detecta la influencia del borde sobre las partículas adyacentes a éste. Asumiendo el simple caso que se trata de un estado, es decir no hay estados metaestables como predice teoría de primer orden aleatorio (RFOT), el autosolapamiento decae de un alto valor $A$ cerca del borde a $q_{0}$ cerca del centro de la cavidad. De esta manera, el autosolapamiento puede ser expresado como una función del radio de la cavidad [Cavagna et al., 2007]. Para ello, dividimos una esfera de radio $\mathrm{K}$ en cascarones esféricos de radio $\mathrm{r}$ :

$$
\mathrm{Q}_{\infty}(\mathrm{K})=\frac{1}{\frac{4}{3} \pi \mathrm{K}^{3} \rho} \int_{0}^{\mathrm{K}} \mathrm{dr} \frac{4 \pi \mathrm{r}^{2}}{1^{3}}\left\langle\mathrm{Q}_{\infty}(\mathrm{r})\right\rangle=\frac{3}{\mathrm{~K}^{3}} \int_{0}^{\mathrm{K}} \mathrm{drr}{ }^{2} \mathrm{G}(\mathrm{r})
$$

Donde $\mathrm{G}(\mathrm{r})=\left\langle\mathrm{Q}_{\infty}(\mathrm{r})\right\rangle / \mathrm{q}_{0}$ representa el autosolapamiento promedio por unidad de volumen, entre un estado $\alpha$ y el estado asintotico congelado. Si asumimos que el efecto del borde implica que $\mathrm{G}(\mathrm{r}=\mathrm{K})=1$, entonces seria razonable proponer

$$
G(r)=\left(A-q_{0}\right) e^{-(K-r) / \xi}+q_{0}
$$

De manera que si insertamos $\mathrm{G}(\mathrm{r})$ en la ecuación 5.10, obtenemos la expresión para un estado:

$$
\mathrm{Q}_{1 \mathrm{~S}}(\mathrm{~K})=3\left(\mathrm{~A}-\mathrm{q}_{0}\right)\left[\frac{1}{x}-\frac{2}{x^{2}}+\frac{2\left(1-\mathrm{e}^{-\mathrm{x}}\right)}{\mathrm{x}^{3}}\right]+\mathrm{q}_{0},
$$

Con $x=K / \xi$, donde la longitud de penetración $\xi$ debe ser proporcional a $\xi_{\mathrm{PTS}}$ en este escenario. Un ajuste de esta expresión, presentado en la figura 5.12, presenta una longitud de penetración que se comporta similarmente, aunque quizas mas suavemente, a la correlación PTS $\xi_{\text {PTS }}$ (ver Figura 5.13).

Puesto que el decaimiento de PTS es (siempre) exponencial y que el decaimiento del autosolapamiento $Q$ puede ser explicado por el ansatz de 1-estado ( $\left.\mathrm{Q}_{1 \mathrm{~S}}\right)$, no parece que nos encontremos en un escenario como el propuesto por RFOT. Si estudiamos $\tau$ vs $\xi$, ver Figura 5.14, observamos que en vez de un comportamiento exponencial, nos encontramos con un comportamiento de ley de potencia. Sin embargo, observamos que los exponentes hallados $\zeta$, se encuentran muy por arriba de lo que corresponde a exponentes críticos dinámicos.

Nuestros hallazgos dan una imagen que no apunta a la de un comportamiento vítreo sino, quizás, a la de un sistema que experimenta una transición de fase. Volveremos sobre esto en las conclusiones en la sección 10. 

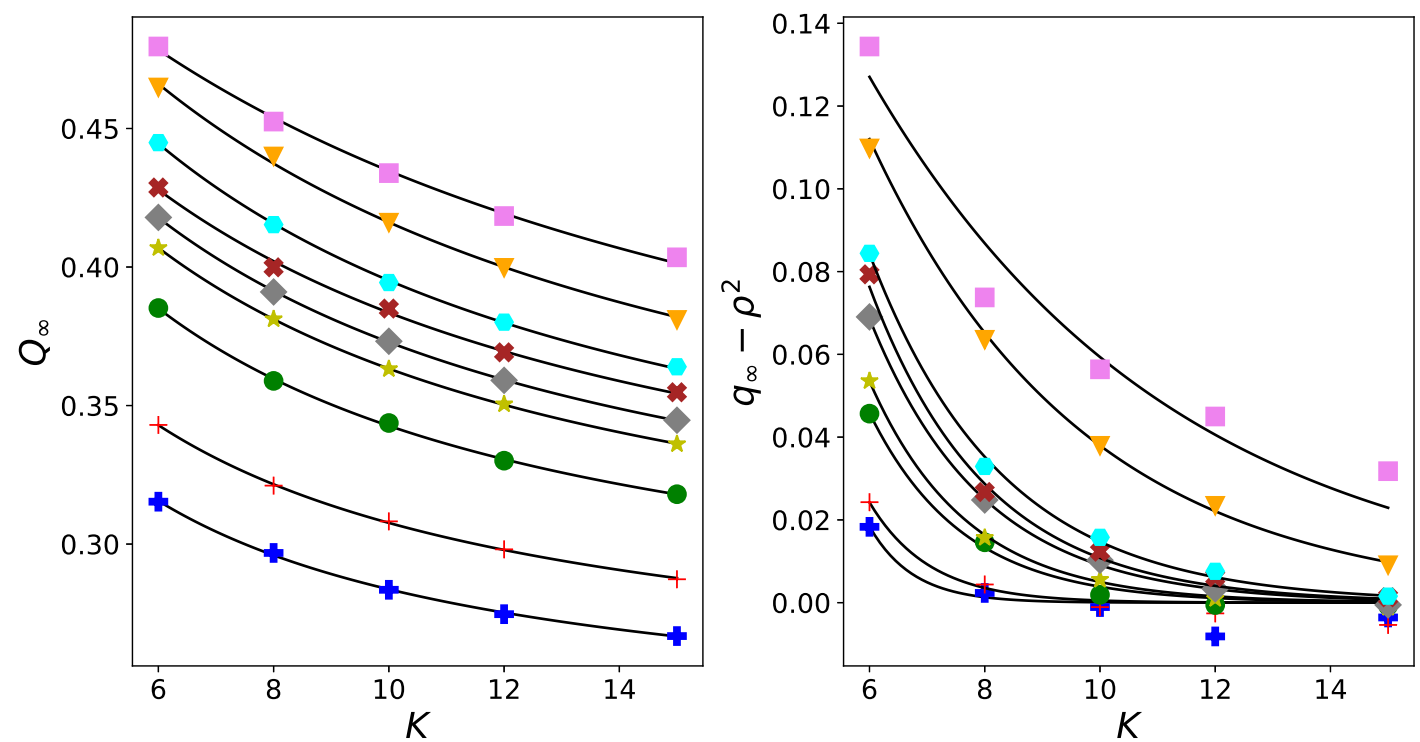

Figura 5.12: De abajo hacia arriba, $\rho=0.48,0.493,0.51,0.518,0.522,0.526,0.5296,0.535,0.54$. El valor estático $\mathrm{Q}_{\infty}(\mathrm{K})$ fue ajustado usando $\mathrm{Q}_{1 \mathrm{~s}}(\mathrm{~K})$ de la ecuación 5.12, mientras que el valor asintótico PTS $q_{\infty}(K)$ fue ajustado usando $q_{c}(K)$ de la ecuación 5.9.

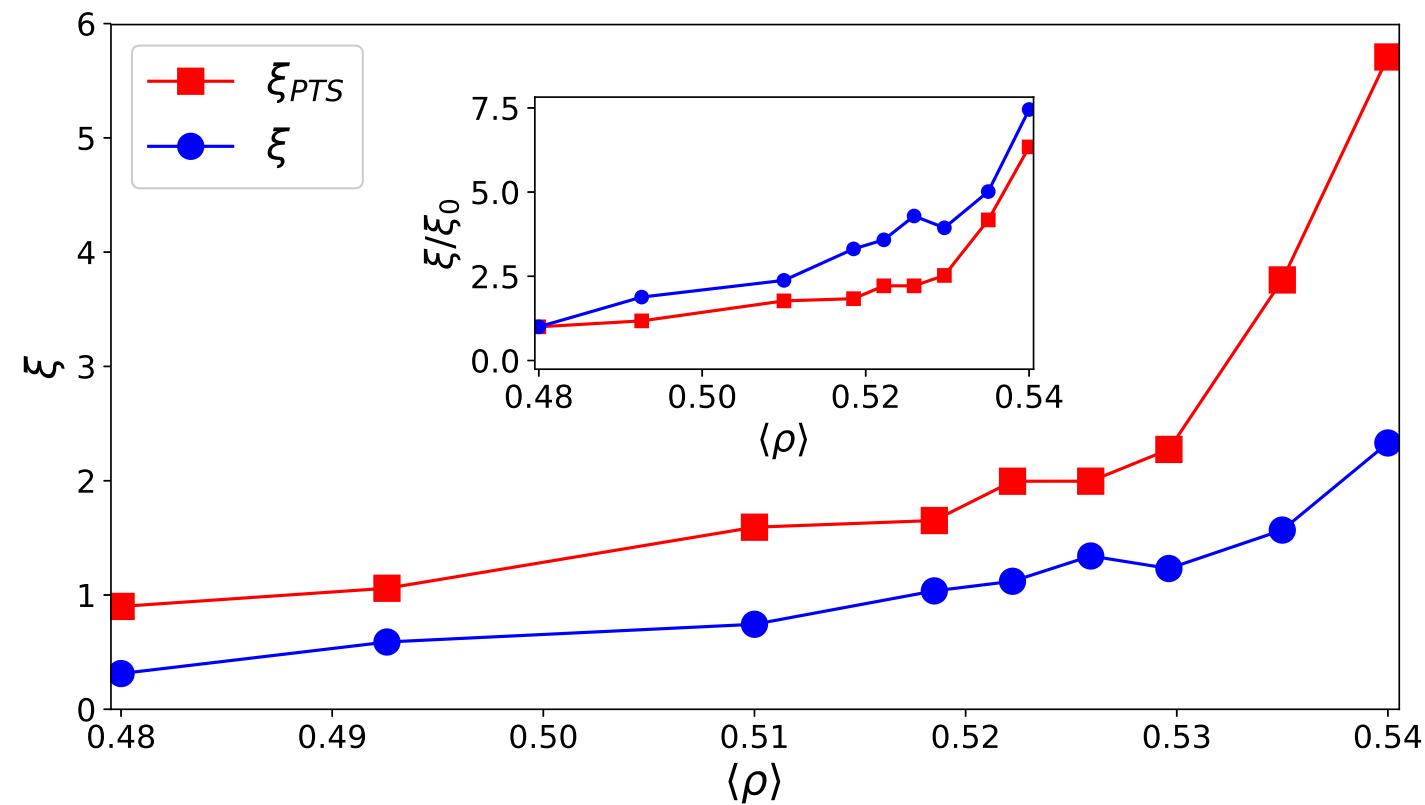

Figura 5.13: Longitud de correlación そ para el autosolapamiento Q y la relajación PTS q. Ambas presentan un suave incremento que duplica su valor inicial, sin embargo, para $\rho>0.53$, PTS presenta un súbito crecimiento. RECUADRO: Longitud de correlación normalizada usando $\xi_{0}=\xi(\rho=0.48)$. 

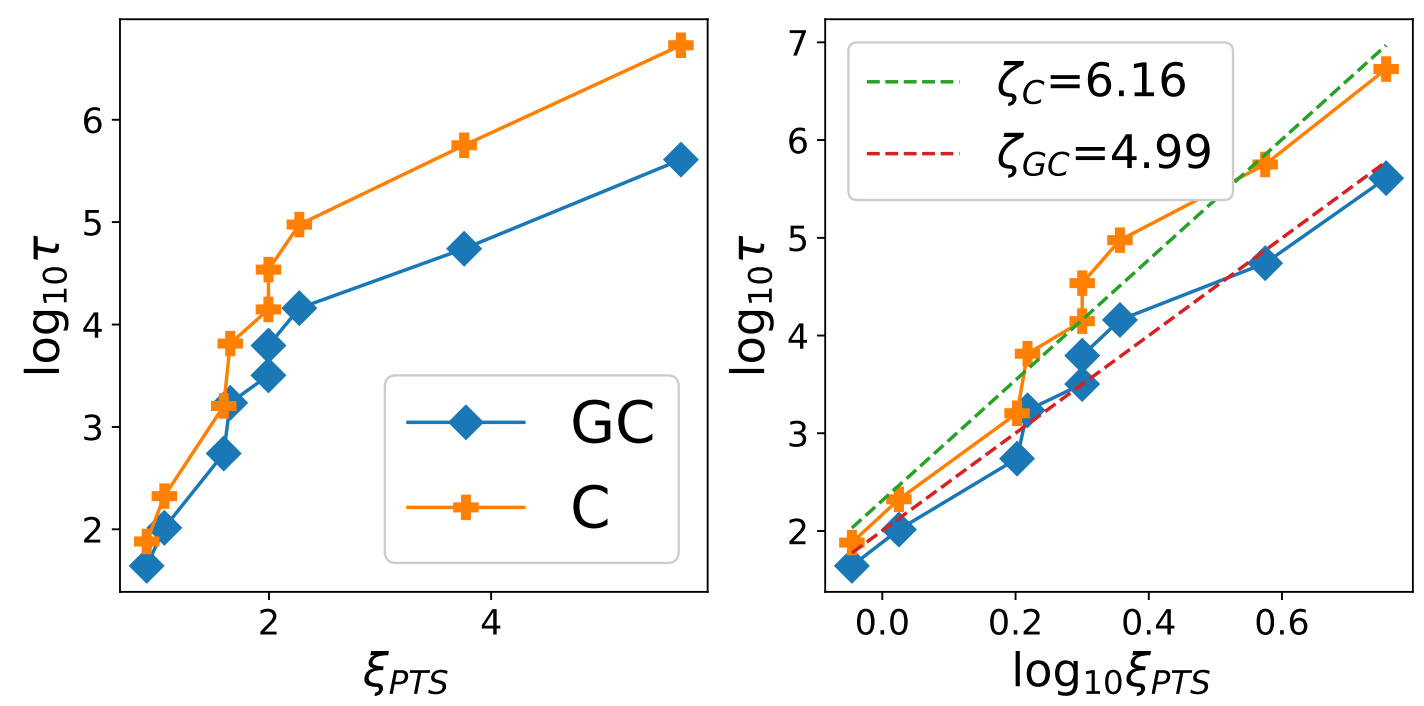

Figura 5.14: Tiempo de relajación $\tau$ para ensambles canónicos y GC en función de la longitud de correlación estática $\xi_{\text {PTS }}$. Observamos un comportamiento de ley de potencia con exponentes $\zeta$. 
Parte II

\section{Partículas Autopropulsadas}




\section{Capítulo 6}

\section{Introducción a la Materia Activa}

Los sistemas fuera de equilibrio aparecen bajo un amplio espectro de situaciones con fenomenologías muy variadas. En la sección 2 presentamos sistemas vítreos que están relajando hacia un equilibrio térmico, pero el cual eventualmente parecen nunca alcanzar. Si bien la relajación puede ser extremadamente lenta, existe una dirección o tendencia en la que el sistema evoluciona, o que evolucionaría si le fuese posible.

En el resto de este trabajo, nos volcaremos hacia otra clase de sistemas fuera del equilibrio, denominados sistemas de materia activa [Ramaswamy, 2010]. La característica que unifica a esta gran variedad de sistemas de materia condensada, sea materia viva o imitaciones de esta, es la capacidad de autopropulsarse convirtiendo energía almacenada, o energía libre del ambiente, en movimiento sistemático [Schweitzer, 2007]. Cada elemento individual que constituye el sistema, presenta una dinámica irreversible. La interacción de partículas activas entre sí y con el medio en que viven da lugar a esfuerzos mecánicos y movimientos colectivos altamente correlacionados.

Los sistemas de partículas activas presentan una variedad de propiedades del noequilibrio [Marchetti et al., 2013]. Éstos poseen estructuras emergentes con movimiento colectivo cualitativamente diferente del de sus constituyentes individuales, transiciones fuera del equilibrio de orden-desorden, formación de patrones en escalas mesoscópicas, propagación de ondas y oscilaciones sostenidas, aun en ausencia de inercia en el sentido estricto.

Esto incluye una gran variedad de sistemas cuyas partículas se desplazan. Ejemplos comunes son: bandadas de pájaros [Vicsek et al., 1995], cardúmenes de peces [Par, 1997], propiedades mecánicas del citoesqueleto como un gel activo [Julicher et al., 2007], células de tejido [Szabó et al., 2006], entre muchos otros.

Si bien muchos de estos sistemas han sido estudiados en gran profundidad, muchas veces desde el campo de la biología/zoología, quizás el trabajo [Vicsek et al., 1995] de 


\begin{tabular}{|c|c|c|}
\hline & Nemático & Polar \\
\hline Seco & varas granulares vibradas & animales migrantes \\
\hline Mojado & varas en suspensión & citoesqueleto de una célula \\
\hline
\end{tabular}

Cuadro 6.1: La tabla presenta ejemplos de las 4 clases de universalidad propuestas en función de simetría y presencia/ausencia de conservación del momento (mojado/seco).

Vicsek y colaboradores, donde presentan el comportamiento de bandadas como una transición de fase orden-desorden, dio uno de los puntapié iniciales para la búsqueda de una descripción genérica de materia activa desde la física estadística.

Es llamativo que un sistema de partículas autopropulsadas con interacciones simples, presenta una variedad de comportamientos de interés. Resulta razonable pensar que estos sistemas puedan formar una subclase de los sistemas fuera del equilibrio con un marco teórico común.

\subsubsection{La Imposibilidad de una Descripción Teórica General para Ma- teria Activa}

En su trabajo [Marchetti et al., 2013], los autores discuten la imposibilidad de (por el momento) sortear la complejidad de describir las propiedades generales de la materia viva. En un dado organismo vivo hay como máximo, 300 tipos distintos de células, el cual es un número pequeño dada la inmensidad del espacio de parámetros accesible.

Quizás, el enfoque sobre principios globales tales como las leyes de conservación y simetrías, sean las que determinen los posibles comportamientos dinámicos de células, organismos o poblaciones de bacterias [Thompson et al., 2011], cardúmenes o bandadas de pájaros. Cuantificar la organización dinámica espontánea y el movimiento de sistemas vivos puede ser un primer paso hacia el entendimiento, de manera genérica, de algunos de estos principios. La esperanza está puesta en poder clasificar la materia activa en un pequeño número de clases de universalidad, basándose en consideraciones de simetría y leyes de conservación, cada una con un comportamiento macroscópico bien definido.

En su trabajo [Marchetti et al., 2013] presenta 4 clases (ver cuadro 6.1) de materia activa de acuerdo a la naturaleza de simetría rota de la fase ordenada y el tipo de disipación de la cantidad de movimiento. Hacemos referencia a algunos de los sistemas que el cuadro pretende clasificar: En su trabajo [Gruler et al., 1999], Gruler y colaboradores estudian la habilidad de células interactuantes para formar fases de cristal liquido. Resaltando la distinción polar/apolar para materia viva. Esta distinción suele estar 
asociada a una simetría rota. Los objetos elongados autopropulsados son, en general, entidades polares con cabeza y cola bien definida. En una fase polar, todos los objetos microscópicos están, en promedio, alineados en la misma dirección. El orden polar esta descrito por un parámetro de orden vectorial $\vec{p}$, llamado polarización. Por otra parte, ordenamiento nemático puede ser obtenido tanto en sistemas donde objetos polares (cabeza-cola) autopropulsadas se ordenan de forma paralela o bien en partículas que resultan simétricas en cabeza-cola, como los melancocitos que distribuyen pigmento en la piel [Marchetti et al., 2013].

\subsubsection{Bandadas}

Es importante hacer la distinción, de que la materia activa se ocupa (en parte) de la mecánica estadística de la materia viva [Ramaswamy, 2010]. El aspecto interesante de la materia biológica es la habilidad de traducir energía libre en movimiento sistemático. Es ésta la propiedad que define a la materia activa y a las propiedades mecánicas que surgen de dicho movimiento. Si consideramos esto, junto con el movimiento colectivo de grandes números de constituyentes, resulta natural considerar una bandada coherente de pájaros, bestias o bacterias como una fase orientacionalmente ordenada de materia viva.

En un estudio hidrodinámico [Toner and Tu, 1995], Toner y colaboradores brindan una teoría general de bandadas. En su trabajo titulado Órden de largo alcance en un modelo XY dinámico bidimensional: Como los pájaros vuelan juntos ${ }^{1}{ }^{1}$, los autores discuten un modelo continuo para el movimiento colectivo de grupos de grandes números de organismos biológicos, dentro de cuya clase de universalidad incluye al trabajo de [Vicsek et al., 1995].

En vez del enfoque hidrodinámico, podemos optar por un modelo de agentes. Los modelos basados en agentes ofrecen un enfoque mínimo para el estudio de sistemas activos, con un énfasis en el orden y fluctuaciones, en vez de fuerzas y mecánica. Estos modelos [Vicsek and Zafeiris, 2012], describen partículas puntuales con velocidad constante moviéndose sobre un sustrato inerte. La dirección del movimiento cambia de acuerdo a una regla de ruido local que requiere alinearse con sus vecinos para cada instante del tiempo. Esta familia de modelos exhibe una transición bien definida de desorden-orden al disminuir la fuerza del ruido o incrementar la densidad. En particular, en los estudios del modelo de Vicsek [Vicsek et al., 1995] (ver sección 6.4.2), existe una controversia respecto al orden de la transición [Grégoire and Chaté, 2004]

\footnotetext{
${ }^{1}$ Titulo original en ingles: 'Long-Range Order in a Two-Dimensional Dynamical XY Model: How Birds Fly Together'
} 
[Baglietto and Albano, 2009]. Los autores estudian la transición de orden-desorden, exponiendo que efectos de condiciones de borde y tamaño finito son los que causan la controversia.

Mas allá de la controversia, resulta claro que el estudio de movimiento colectivo para partículas auto-propulsadas es un campo rico en aplicaciones para la mecánica estadística. Desde un punto de vista puramente físico, el fenómeno espontáneo de formación de bandadas sin líderes, campos externos o geometrías que restringen el proceso puede ser dicho de otra manera: La ruptura espontánea de una simetría continua, donde vemos el movimiento colectivo de la bandada como una fase orientacionalmente ordenada de materia activa. Es decir, la formación de una bandada puede ser asociada a una transición de fase fuera del equilibrio.

\subsection{Transiciones de Fase fuera del Equilibrio}

Así como en la sección 2, el estudio de sistemas de materia activa nos aleja de los sistemas en equilibrio termodinámico. Para sistemas en equilibrio termodinámico, es posible definir estados en equilibrio macroscópico, como aquellos que resultan simétricos a inversiones y translaciones temporales. Esto requiere que sean estados macroscópicamente estacionarios y que no existan flujos macroscópicos netos de ningún tipo (materia, energía, etc.) sosteniéndolos.

En cambio, estados fuera de equilibrio pueden exhibir flujos de masa y/o energía, que pueden ser generados por condiciones de contorno (espaciales y/o temporales), por campos externos dependientes del tiempo o ambos [Racz, 2002]. Esto lleva a que los estados fuera del equilibrio no pueden quedar unívocamente caracterizados por parámetros externos, sino que además dependen de la historia del sistema. Sin embargo, estos parámetros son suficientes para especificar el estado cuando los flujos son estacionarios (el caso mas simple).

Las variaciones de parámetros externos pueden desestabilizar un estado fuera del equilibrio y causar un cambio de estado. Este puede ser sucedido por un fenómeno de ordenamiento análogo al que ocurre con sistemas en equilibrio. Estos cambios son llamados transiciones de fase fuera del equilibrio [Marro and Dickman, 2005] [Cross and Hohenberg, 1993].

Sin embargo, a diferencia de sus contrapartes en equilibrio, las transiciones de fase fuera del equilibrio no poseen un marco teórico bien establecido. Esto lleva a que el progreso en esta rama de la física se base en gran proporción en el estudio y comprensión de modelos simples. En muchas ocasiones, la descripción de sistemas fuera del equilibrio se hace prescindiendo de una formulación hamiltoniana. Ya sea por que no se cuenta 
con una (o resulta impráctica), la consecuencia directa de la ausencia del hamiltoniano es la ausencia de una energía libre con la cual podamos definir precisamente el orden de las transiciones de fase.

Para suplir esta carencia, la presencia de fenómenos críticos (escaleo, exponentes críticos, etc.) y el cambio continuo o discontinuo del parámetro de orden en los puntos de transición son los criterios que definen la transición [Loscar and Albano, 2003].

\subsection{Complejidad y Fenómenos Emergentes}

La emergencia ${ }^{2}$ de ciencia no-lineal sobre fines del siglo 20, atrajo interés en la vieja idea de que explicaciones causales no siempre pueden ser completamente reducidas a correspondencias uno-a-uno entre causa y efecto. Las cosas complejas se suelen autoorganizar como patrones de alto nivel, mediante procesos de interacciones locales entre entidades simples. La emergencia del todo (comportamiento colectivo de las unidades) a partir de tal no linealidad, hace que la capacidad de predicción para la evolución de un sistema sea muy escasa [Enc, 2004]. Un sistema complejo está compuesto por varias partes interactuantes, cuyas interacciones crean información adicional no visible al analizar las partes de forma individual. Estos sistemas suelen resultar difíciles, sino imposibles, de resolver exactamente. El ejemplo mas notorio es el de un sistema de tres partículas con interacciones gravitatorias [Poincaré, 1891].

Gran parte de la atención en el estudio de estos sistemas se centra en las denominadas propiedades emergentes. Estas son propiedades globales que le dan al sistema una identidad propia, es decir, las propiedades emergentes de un sistema manifiestan no tanto la base material de sus componentes (pájaros, peces) sino la organización del material (bandadas, cardumenes).

La complejidad que emerge de los sistemas complejos no necesariamente se debe a que las interacciones entre los constituyentes sean complejas, sino a que no se las puede despreciar y por que conducen a que el ¡comportamiento del sistema como un todo sea cualitativamente diferente al de los constituyentes.

La simplicidad de las interacciones, nos permite tratar una variedad de problemas de ramas como la biología o finanzas, pues suponemos se trata de sistemas de partículas interactuantes. Estas partículas pueden ser predadores y presas o agentes del mercado financiero. Naturalmente se trata de partículas muy complejas, pero la aproximación de sistemas de partículas interactuantes, pone el enfoque en las interacciones efectivas entre ellas, despreciando su estructura interna.

\footnotetext{
${ }^{2}$ Usamos emergencia, en el sentido de algo que emerge o surge.
} 
Evitando una descripción detallada de las partículas, podemos estudiar al sistema buscando entender la complejidad que emerge de algún tipo particular de interacción entre las partes del mismo.

Para trazar una conexión con los sistemas vítreos, podemos considerar el argumento de paisaje de energía (ver sección 2.6.1). Los estados de un sistema, tienen la misma estructura que los mínimos de su hamiltoniano [Castellani and Cavagna, 2005]. El número de mínimos $\mathcal{N}$ crece exponencialmente con el tamaño del sistema $\mathrm{N}$.

$$
\mathcal{N} \sim e^{N \Sigma}
$$

$\Sigma$ es llamada complejidad (o entropía configuracional). Sea E la densidad de energía podemos calcular $\Sigma$ haciendo:

$$
\Sigma(E)=\lim _{N \rightarrow \infty} \frac{1}{N} \log \mathcal{N}(E)
$$

Lo que puede interpretarse como que la complejidad, corresponde al logaritmo del número de mínimos (pozos) en el paisaje de energía. Considerar los grados de libertad discretos (número de partículas) y continuos (sus posiciones), junto con fenómenos emergentes debidos a las interacciones colectivas, origina la complejidad de estos paisajes de energía.

\subsection{Fuerzas Intermoleculares y Potenciales Modelo}

Podemos tomar como punto inicial de la discusión sobre las propiedades de una substancia en distintas fases, a la relación entre presión $\mathrm{P}$, densidad $\rho=\mathrm{N} / \mathrm{V}, \mathrm{y}$ temperatura $T$, explicitada en $f(P, \rho, T)=0$ [Hansen and McDonald, 1990].

Para la fase líquida, la interacción definitoria en el comportamiento entre partículas, es el de la repulsión que aparece a corto rango. Las fuerzas atractivas, si bien actúan a largo alcance, varían mucho más suavemente con la distancia y juegan un rol menor en la determinación de la estructura de un líquido.

Separar los efectos de repulsión de los de atracción tiene raíces en las ideas de van der Waals. El modelo mas sencillo para representar un fluido mediante la interacción entre partículas separadas por una distancia $r$, modelada por un potencial de pares $\mathrm{V}(\mathrm{r})$, es:

$$
V(r)= \begin{cases}\infty & \text { para } r \leqslant d \\ 0 & \text { para } r d\end{cases}
$$

Donde la ecuación 6.3 representa el potencial de esferas duras, con un diámetro d. Este 
potencial representa la rigidez total de las partículas. Si bien este potencial incluso exhibe una transición de cristalización ( para $\rho \mathrm{d}^{3} \simeq 0.945$ ), la ausencia de fuerzas atractivas implica la existencia de una única fase de fluido.

Un potencial capaz de describir un verdadero líquido puede ser obtenido suplementando el potencial de esferas duras, con un término de atracción. Un potencial que además considera atracción y con características de interés teórico, es el potencial de Yukawa [Hansen and McDonald, 1990]:

$$
V(r)= \begin{cases}\infty & \text { para } r<d \\ -\frac{\epsilon d}{r} \exp \left[-\frac{\lambda}{d}(r-d)\right] & \text { para } r>d\end{cases}
$$

Donde $€$ y $\lambda$ son parámetros que caracterizan la atracción.

Sin embargo, un potencial aún más realista, basado en una construcción mecánicocuántica para átomos neutros, es el potencial propuesto por John Lennard-Jones [Hansen and McDona Este potencial considera las contribuciones dominantes de las interacciones de dispersión multipolar entre momentos eléctricos en átomos.

Si bien el comportamiento resulta sumamente complicado, los casos limites cuando $r \rightarrow 0$ y $r \rightarrow \infty$ pueden agruparse en la simple expresión:

$$
\mathrm{V}_{\mathrm{LJ}}(\mathrm{r})=4 \epsilon\left[\left(\frac{\sigma}{\mathrm{r}}\right)^{12}-\left(\frac{\sigma}{\mathrm{r}}\right)^{6}\right]
$$

En esta expresión, $\sigma$ representa el diámetro de colisión, es decir la separación de partículas cuando $\mathrm{V}_{\mathrm{LJ}}(\mathrm{r})=0 \mathrm{y} \in$ representa la profundidad del pozo de potencial en el mínimo de $\mathrm{V}_{\mathrm{LJ}}(\mathrm{r})$.

Este potencial provee una descripción de la interacción de gases nobles, así como una buena representación para átomos y moléculas esféricas neutras a corta y larga distancia de interacción. El ordenamiento de partículas de menor energía es el empaquetamiento hexagonal. De incrementar la temperatura, el empaquetamiento que minimiza la energía libre es el FCC, para luego pasar a fase líquida.

Nos preguntamos si un sistema binario de partículas autopropulsadas, con alguna variante de los potenciales presentados, da lugar a una transición de segregación. En su trabajo [Jungblut and Dellago, 2011], Jungblut y colaboradores estudian una mezcla binaria con interacción Lennard-Jones. En este caso, las partículas difieren únicamente en diámetro, lo que produce un enlentecimiento de un orden de magnitud en el crecimiento cristalino.

Por otra parte, Huang y colaboradores [Huang et al., 2006], presentan una separación de fase en un fluido binario bidimensional modelado con una variación del potencial 


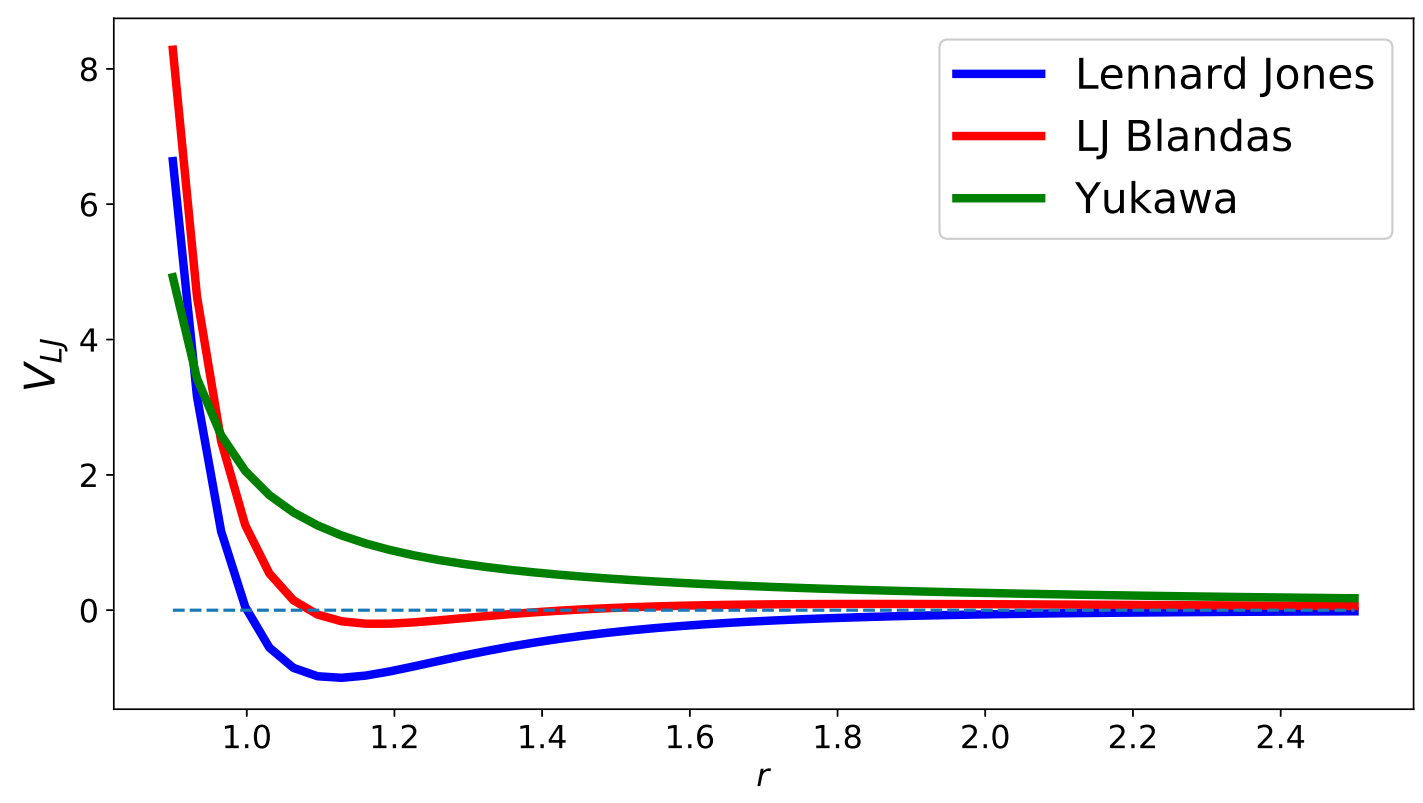

Figura 6.1: Representación esquemática para los potenciales mencionados. Si bien el comportamiento cuando $\mathrm{r} \rightarrow 0$ y $\mathrm{r} \rightarrow \infty$ es similar, los distintos potenciales varian su comportamiento en la zona intermedia. Valores usados: $\epsilon=1, \epsilon^{\prime}=1.2, \sigma=1, \mathrm{~d}=1, \lambda=$ 0.1 .

de Lennard-Jones, el núcleo blando U(r):

$$
\mathrm{U}(\mathrm{r})=4 \epsilon\left[\left(\frac{\sigma}{\mathrm{r}}\right)^{12}-\left(\frac{\sigma}{\mathrm{r}}\right)^{6}\right]+\epsilon^{\prime}\left(\frac{\sigma}{\mathrm{r}}\right)^{3}
$$

El potencial resulta puramente repulsivo, presentando segregación en cumulos ${ }^{3} \mathrm{o}$ total en función de la concentración. Concluyen, que la diferencia de tamaño en las partículas, es un factor importante en la determinación de las distintas partes de espacio de fase del sistema. Presentamos los distintos potenciales, de manera esquemática, en la figura 6.1

Otro caso [Redner et al., 2013] de partículas activas con volumen excluído sin interacción orientacional, es el que presenta Redner y colaboradores. El fluido coloidal fuera del equilibrio estudiado por ellos, se separa en una fase densa y una diluida. Dicha fase densa, la cual ellos denominan sólido activo, exhibe evidencia estructural de ser un sólido cristalino, así como movimiento superdifusivo en escalas temporales intermedias.

En su trabajo [Bialké et al., 2013], Bialké y colaboradores estudian un sistema bidimensional de discos autopropulsados sin interacción orientacional. En este, la inestabilidades dinámicas producen una separación de fase y requieren que el sistema no

${ }^{3}$ Por cumulo nos referimos a la palabra inglesa cluster 
alcance el equilibrio térmico.

Estos trabajos señalan la presencia de una separación de fase por movilidad en sistemas de mezclas de partículas activas, ya sea con volumen excluído o una variación del potencial de Lennard Jones.

\subsection{Interacciones Orentacionales}

Es llamativo el surgimiento de un ordenamiento colectivo sin la presencia de lideres de ningún tipo, gradientes en el medio o confinamiento geométrico [Couzin et al., 2005]. Enfocado desde la materia condensada, consideramos algunos modelos físicos para presentar el modelo orientacional a estudiar.

\subsubsection{El Modelo XY}

El modelo tradicional para el estudio de transiciones de fase, es el modelo de Ising [Goldenfeld, 1992].

Observamos se trata de un modelo no lineal, debido a las interacciones entre espines. Allí radica precisamente la existencia del fenómeno cooperativo que es la transición de fase. Si bien el modelo de Ising, presenta interacción entre partículas y orientaciones, este aun resulta demasiado limitado para poder modelar el comportamiento de una bandada de aves o un cardúmen de peces. Un paso mas en complejidad, seria permitir que los espines $s_{i}$ sean capaces de apuntar a cualquier dirección en el plano. Esto nos lleva al modelo XY [Goldenfeld, 1992] [Toner and Tu, 1995].

El modelo $X Y$ posee el mismo hamiltoniano que el modelo de Ising. Al brindarles simetría O(2) a los espines, transformándolos en vectores unitarios capaces de rotar en el plano, estos resultan una mejor aproximación del ordenamiento de una bandada de aves en el cielo. Sin embargo, la elección del modelo XY para representar fenómenos de transporte colectivo de partículas autopropulsadas no es correcta, dado que la posición de los espines esta fija en el espacio.

\subsubsection{El Modelo de Vicsek}

Tamas Vicsek y colaboradores, introdujeron [Vicsek et al., 1995] un modelo mínimo pero capaz de capturar de forma no trivial las principales características del movimiento colectivo. El modelo de Vicsek (MV) puede ser descrito como el estudio del surgimiento de orden orientacional de largo alcance a traves de una ruptura espontánea de la simetría en sistemas de partículas autopropulsadas [Vicsek and Zafeiris, 2012, Czirók et al., 1996]. 
En el MV, partículas puntuales se mueven con módulo de velocidad fija, tratando de alinearse con sus vecinas mientras experimentan ruido orientacional. El modelo presenta tanto una fase ordenada de transporte neto de materia (bajo ruido) y una fase gaseosa desordenada (alto ruido).

Nuevamente basándonos en el magnetismo, el observable comunmente usado para el estudio de sistemas con orden orientacional, es la magnetización ${ }^{4} \Phi$. Ésta viene dada por la ecuación:

$$
\Phi=\frac{1}{N}\left|\sum_{i}^{N} \vec{v}\right|
$$

De esta manera, si todas las partículas apuntan en la misma dirección, $\Phi$ vale 1. Si las partículas se encuentran apuntando en direcciones aleatorias, $\Phi$ debe valer 0 . Este parámetro de orden es usado ampliamente en los estudios de materia activa [Marchetti et al., 2013] y da una medida del orden emergente del movimiento colectivo del sistema.

\subsection{Objetivos de este trabajo}

El equilibrio termodinámico establece una distribución de Boltzmann. Esto implica que el estado estacionario debe ser independiente de los parámetros cinéticos. Para las partículas autopropulsadas, este no es el caso. Las partículas autopropulsadas consumen energía continuamente. De esta manera se ven desafectadas de las leyes de la termodinámica de equilibrio. Éstas tienden a acumularse a medida que se mueven mas lentamente [Cates and Tailleur, 2015]. También pueden enlentecerse debido a alta densidad, tanto por razones estéricas como bioquímicas. Este comportamiento, puede llevar a una separación de fase inducida por la propia movilidad, entre fases densas y diluidas.

Por otra parte, como presenta [Huang et al., 2006], las mezclas binarias pasivas bidimensionales presentan distintos tipos de segregación en función de concentración y diferencia de tamaño. Un primer paso para considerar las diferencias con el caso pasivo, es el de tomar partículas activas esféricas autopropulsadas, cuyas interacciones son isotrópicas.

Aun considerando interacciones de atracción y repulsión, sin interacciones orientacionales, la fenomenología presente en estos sistemas de fluidos simples (pero activos) resulta mucho mas rica y compleja que la de sus contrapartes pasivas [Levis and Berthier, 2014] [Thompson et al., 2011].

En su trabajo [Fily and Marchetti, 2012], Fily y colaboradores, presentan un estu-

\footnotetext{
${ }^{4}$ Aveces también llamado polarización
} 

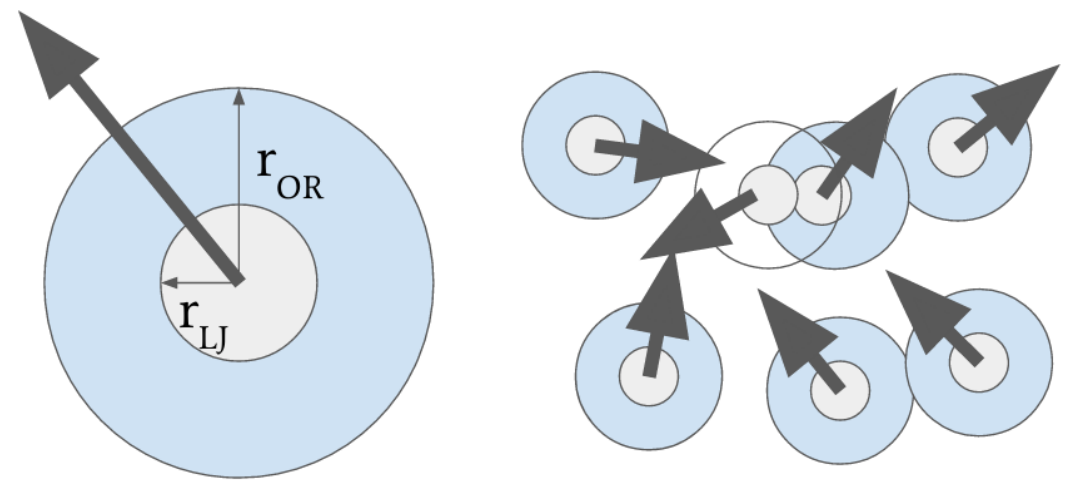

Figura 6.2: Representación de los rangos de las interacciones entre partículas. Notar que partículas separadas a distancia mayor que el radio orientacional $\left(\mathrm{r}_{\mathrm{OR}}\right)$ no experimentan ninguna interacción y se mueven libres. Cuando las partículas se aproximan a una distancia menor a $r_{\mathrm{LJ}}$ comienzan a experimentar repulsión Lennard Jones.

dio de discos autopropulsados en un espacio bidimensional, interactuando únicamente mediante fuerzas repulsivas isotrópicas y el agregado de ruido rotacional. El sistema exhibe el comportamiento de grumo típico de partículas activas.

Nuestro objetivo es el de caracterizar un sistema binario activo, de partículas con volumen excluido. Las partículas solo difieren en su velocidad, clasificandolas como rápidas o lentas. Buscamos determinar si el volumen excluido, modelado por una interacción Lennard Jones truncada (solo repulsión), da lugar a una transición de segregación como la observada para un sistema pasivo por [Rovere et al., 1990]. Denotamos a este modelo como LJ, debido a que solo contempla interacciones Lennard Jones truncadas.

Este sistema canónico nos brinda parámetros como la composición, densidad y velocidad de las partículas, para estudiar formación de dominios y segregación.

Excluimos el ruido orientacional de todos los estudios, puesto que el propio volumen excluído y el carácter binario del sistema ofrecen ya suficientes restricciones para evitar que el sistema alcance un régimen estacionario trivial.

Una vez caracterizado el modelo LJ, activamos las interacciones orientacionales que dan lugar al comportamiento de enjambre en conjunto con la interacción del volumen excluído. Volveremos a estudiar la naturaleza de la segregación al incorporar interacción orientacional. Presentaremos las metodologías utilizadas en el capítulo 7. 


\section{Capítulo 7}

\section{Simulaciones II: Metodología Utilizada en el Estudio de Materia Activa}

\subsection{Sistemas Continuos}

Si bien algunas diferencias entre sistemas en el retículo y sistemas continuos fueron presentadas en el capítulo 3, debemos mencionar que resulta imposible lidiar con números reales con infinita precisión en una computadora. Este problema no existe para números enteros.

Notamos que números irracionales u otros ciertos números reales no enteros, no pueden ser representados con sus infinitas cifras por una computadora. Los números reales de los que disponemos poseen una cierta precisión, es decir un cierto numero de dígitos. Valiéndonos de la coma flotante, podemos representar números racionales extremadamente grandes y pequeños de una manera muy eficiente y compacta, y con la que se pueden realizar operaciones aritméticas.

El uso de unidades adimensionales nos brinda la posibilidad de trabajar con valores del orden de la unidad, en vez de los valores típicamente muy pequeños, asociados a la escala atómica, o valores muy grandes, asociados escalas astronomicas [Rapaport, 2004].

Estas unidades implican no solo una simplificación de las ecuaciones de movimiento, sino la posibilidad de escalear los resultados para toda una clase de sistemas descritos con el mismo modelo. Es en este marco que seleccionaremos los valores de los parámetros de las simulaciones de dinámica molecular. 


\subsection{Dinámica Molecular}

La dinámica molecular [Hansen and McDonald, 1990] (DM) es un método para simular un sistema de $\mathrm{N}$ partículas, que poseen coordenadas iniciales dentro de un volumen fijo, usualmente una caja en dos o tres dimensiones. Un conjunto de velocidades iniciales es asignado, típicamente extraído de una distribución de Maxwell, adecuada a la temperatura de interés y seleccionada de manera que el momento lineal neto es nulo.

El calculo subsecuente define el movimiento de las partículas en el espacio por medio de integración de las ecuaciones clásicas de movimiento. Las propiedades de equilibrio pueden ser obtenidas a través de promedios temporales sobre la historia de la dinámica del sistema. Para minimizar efectos de borde, y así simular un comportamiento similar al de un sistema macroscópico, se utilizan condiciones de contorno periódicas.

La DM opera en el continuo, a diferencia de los modelos basados en retículos (como los discutidos en la sección 3). Si bien los modelos de retículo resultan muy efectivos desde un punto de vista computacional, sufren de problemas de diseño tales como limitaciones en el rango de velocidades de las partículas y efectos no deseados debidos a la simetría del retículo [Rapaport, 2004], entre otros.

En sistemas de N partículas, las ecuaciones de movimiento solo pueden ser resueltas numéricamente. En DM, las partículas se mueven en trayectorias determinadas por la solución numérica de las ecuaciones de movimiento, típicamente las ecuaciones de Newton, donde las fuerzas entre partículas y sus energías potenciales son calculadas utilizando potenciales o campos de fuerza.

Debido a la naturaleza de la interacción inter-atómica, ejemplificada por el núcleo repulsivo del potencial de Lennard Jones (ver sección 6.5), las trayectorias son inestables en el sentido de que una perturbación infinitesimal crece a un ritmo exponencial. Esto señala que no todos los problemas, por ejemplo trayectorias atómicas para tiempos moderadamente cortos, son buenos candidatos para la DM. Los errores de integración numérica se van acumulando, e incluso si estos son minimizados mediante una selección apropiada de parámetros y algoritmos, no pueden ser eliminados completamente. Sin embargo, para sistemas bajo la hipótesis ergódica, la evolución de una simulación de DM se puede utilizar para determinar las propiedades termodinámicas macroscópicas del sistema.

\subsubsection{Integración de las Ecuaciones de Movimiento}

Usando las leyes de Newton y considerando un incremento temporal dt suficientemente pequeño (volveremos sobre esto en la sección 7.3.2), podemos pensar el método 
de Euler como el mas directo para resolver las ecuaciones de movimiento:

$$
\begin{aligned}
\vec{r}(t+d t) & =\vec{r}(t)+\vec{v}(t) d t \\
\vec{v}(t+d t) & =\vec{v}(t)+\frac{\vec{F}(t)}{m} d t
\end{aligned}
$$

Donde $\vec{x}, \vec{v}, \vec{F}$ son función del tiempo. Sin embargo, en la literatura, existe una gran variedad de integradores, como el presentado en 7.4, pero que además consideran cuidados adicionales (estabilidad numérica, reversibilidad del tiempo, etc.) a un costo computacional casi idéntico [Rapaport, 2004].

Uno de los integradores mas populares, es el velocity-Verlet que viene dado por:

$$
\begin{gathered}
\vec{r}(t+d t)=\vec{r}(t)+\vec{v}(t) d t+\frac{\vec{F}(t)}{2 m} d t^{2} \\
\vec{v}(t+d t)=\vec{v}(t)+\frac{\vec{F}(t)+\vec{F}(t+d t)}{2 m} d t
\end{gathered}
$$

Resulta crucial el orden de las operaciones en este algoritmo, pues involucra el conocimiento simultaneo de $\vec{v}$ y $\vec{F}$ tanto en $t$ como en $t+d t$. En particular, el orden de las operaciones para el algoritmo velocity-Verlet resulta:

- Dado un estado inicial $\vec{v}(t)$ y $\vec{r}(t)$ a tiempo $t$, primero computar las fuerzas $\vec{F}(t)$ sobre cada átomo.

- Realizar una actualización de posiciones $\vec{r}=\vec{r}+\vec{v} d t+\frac{\vec{r}(t)}{2 m} d t^{2}$.

- Realizar una actualización parcial de las velocidades, usando $\vec{v}=\vec{v}+\frac{F(t)}{2 m} \mathrm{dt}$.

- Calcular las nuevas fuerzas $\vec{F}(t+d t)$ usando las nuevas posiciones $\vec{r}(t+d t)$.

- Completar la actualización de velocidades, usando $\vec{v}=\vec{v}+\frac{\mathrm{F}(\mathrm{t}+\mathrm{dt})}{2 \mathrm{~m}} \mathrm{dt}$.

- Continuar paso siguiente.

\subsubsection{Relación con la Mecánica Estadística}

Hemos visto como utilizar la Dinámica Molecular para calcular trayectorias de partículas que interactúan mediante una fuerza $F(r)$, debida a un potencial $U(r)$. Nuestro interés ahora se vuelca sobre como extraer información de cantidades termodinámicas a partir de dichas simulaciones.

La mecánica estadística se ocupa del promedio sobre ensambles. Para el ensamble canónico, donde la temperatura $\mathrm{T}$ y el número de partículas $\mathrm{N}$ se encuentra fijado, el 
equilibrio de una cantidad $A$ puede expresarse en términos de integrales del espacio de fase con energía potencial $\mathrm{U}\left(\mathrm{r}_{1}, . ., \mathrm{r}_{\mathrm{N}}\right)$ :

$$
\langle A\rangle=\frac{\int A\left(r_{1}, . ., r_{N}\right) e^{-\beta U\left(r_{1}, . ., r_{N}\right) d^{3 N} r}}{\int e^{-\beta U\left(r_{1}, . ., r_{N}\right) d^{3 N_{r}}}}
$$

Donde $r_{i} \in 1, . . N$ son las coordenadas, $\beta^{-1}=k_{B} T$, para $k_{B}$ la constante de Boltzmann. Este promedio corresponde a una serie de medidas sobre un ensamble de sistemas independientes.

La hipótesis ergódica relaciona el promedio de ensambles con medidas llevadas a cabo para un sistema en equilibrio, durante el curso de su evoluciona natural. Ambas deberían producir el mismo resultado [Rapaport, 2004]. Por ello, MD produce el promedio de la forma:

$$
\langle A\rangle=\frac{1}{M} \sum_{\mu=1}^{M} A_{\mu}\left(r_{1}, . ., r_{N}\right)
$$

Donde $M$ son las medidas tomadas mientras el sistema evoluciona. Si el muestreo resulta suficientemente exhaustivo como para capturar el comportamiento típico, ambas promedios deberían ser idénticos.

\subsection{Detalles de optimización en simulaciones DM}

\subsubsection{Potencial Lennard-Jones truncado}

Para ahorrar calculo computacional, el potencial Lennard Jones presentado en 6.5, puede ser truncado. En nuestro caso, como muestra la figura 7.1 elegimos truncarlo en el mínimo de $V_{L J}(r), r_{c}=2^{1 / 6} \sigma$. De esta manera resulta:

$$
\begin{cases}V_{L J}(r)=4 \epsilon\left[\left(\frac{\sigma}{r}\right)^{12}-\left(\frac{\sigma}{r}\right)^{6}\right] & \text { para } r<r_{c} \\ 0 & \text { para } r \geqslant r_{c}\end{cases}
$$

La consecuencia de este truncamiento, resulta en que las partículas son libres para separaciones $r \geqslant r_{c}$, mientras que para $r<r_{c}$ sienten un efecto de repulsión que a medida que $r$ disminuye, la repulsión crece súbitamente. Notar que en el mínimo del potencial, la repulsión se vuelve nula. Esto significa que no hay efectos atracción, los cuales aparecerian para $r>r_{c}$. 


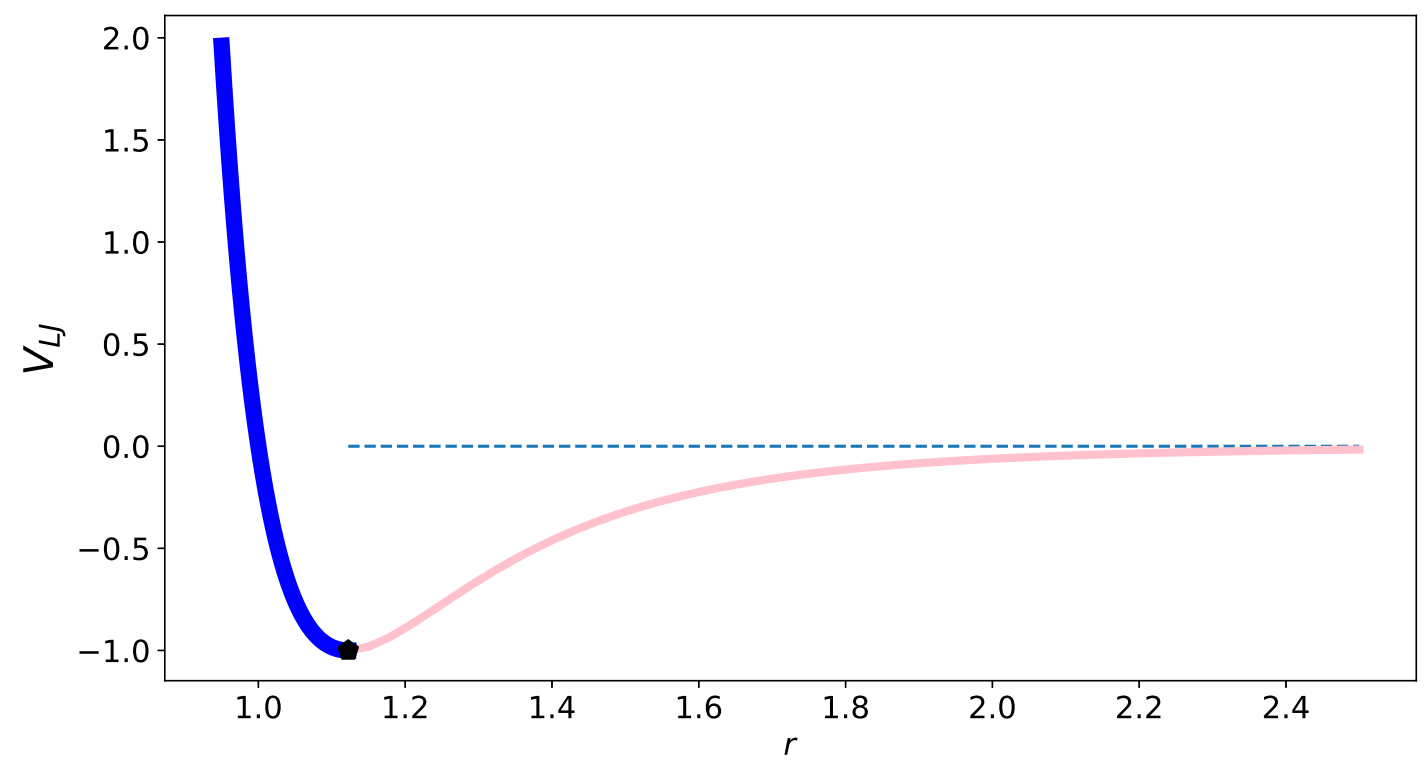

Figura 7.1: El potencial Lennard Jones utilizado, truncado para $r_{c}=2^{1 / 6} \sigma$, donde $\sigma=1$. Observamos repulsión, pero en un rango de alcance limitado.

\subsubsection{Paso temporal de las simulaciones}

Por motivos de estabilidad numérica y precisión, es preciso seleccionar un paso temporal dt aproximadamente un orden de magnitud menor que la escala temporal mas rápida en el sistema [Rapaport, 2004].

Un paso temporal muy grande puede causar que toda la simulación se vuelva inestable con el tiempo. Esto se debe, a que dos partículas pueden evolucionar a posiciones muy próximas en una misma integración. Cuando se realice el nuevo calculo de fuerzas, estas partículas experimentaran una fuerza inmensa que las enviara a una distancia muy grande (que usando condiciones de contorno periódicas, puede ser cualquier sitio de la caja), produciendo un efecto en cadena. Este comportamiento suele ser referido como explosión.

Por otra parte, un paso temporal demasiado pequeño si bien no produce explosiones puede demorar un tiempo total de simulación que el experimentador no esta dispuesto a esperar. Con estos criterios y basándonos en [Yelash et al., 2008],[Das et al., 2003] utilizamos un paso temporal $\mathrm{dt}=0.002$.

\subsection{Efectos de Tamaño Finito}

Ya hemos mencionado en la sección 6.1, que las transiciones de fase de segundo orden se caracterizan por la divergencia de la longitud de correlación $\xi$ en el punto 
critico. De esta manera, distintas cantidades termodinámicas pueden diverger o decrecer fuertemente a medida que nos aproximamos a dicho punto critico.

Las transiciones de fase son fenómenos colectivos cooperativos, lo que significa que solo tienen lugar en sistemas compuestos por partículas que interactúan entre sí. Dicha interacción vuelve al planteo del problema extremadamente complejo, generalmente imposibilitando su resolución analítica. Podemos atacar esta situación mediante el uso de simulaciones numéricas, pero esto también implica el uso de un número finito de partículas. Esto esta en desacuerdo con el hecho de que las transiciones de fase tienen lugar en el limite termodinámico donde, entre otras cantidades, el número de partículas tiende a infinito.

Inmediatamente queda claro que en un sistema finito, resulta imposible hallar una longitud de correlación $\xi \rightarrow \infty$. Las propias dimensiones del sistema acotan el fenómeno crítico, dando lugar a algunas de las siguientes situaciones en el estudio de una transición de fase [Henkel and Pleimling, 2011]:

- $\xi \leqslant \mathrm{L}$, donde L representa una dimensión lineal del sistema finito.

- La susceptibilidad $x$ presenta un pico, no una divergencia.

- La ubicación del valor máximo de $\chi$ puede depender de L

- El parámetro de orden no vale exactamente cero en la fase desordenada, sino que mantiene valores finitos (debido a fluctuaciones estadísticas)

Resulta entonces, que podemos tomar al tamaño finito del sistema L, como una variable mas para el estudio. De esta manera, podemos realizar el estudio de distintas cantidades termodinámicas en función de alguna dimensión lineal L del sistema y observar su tendencia.

\subsubsection{Cumulante de Binder}

La varianza, relacionada a la susceptibilidad $\chi$, es una de las cantidades mas simples y útiles que podemos estudiar usando los momentos de un parámetro de orden. Sea $\phi$ un parámetro de orden, su varianza $\operatorname{Var}(\phi)$ viene dada por:

$$
\operatorname{Var}(\phi)=\left\langle\phi^{2}\right\rangle-\langle\phi\rangle^{2}
$$

Donde $\operatorname{Var}(\phi) \sim \chi_{\phi}$ y $\langle\ldots\rangle$ representa el promedio sobre configuraciones.

Además de estudiar los momentos de primer o segundo orden, podemos ir a ordenes mayores con el fin de extraer información de los efectos de tamaño finito. Un observable 
de utilidad para la determinación del punto crítico en transiciones de fase de segundo orden (continuas), es el cumulante (estático) de Binder de cuarto orden, dado por [Landau and Binder, 2014]:

$$
\mathrm{U}=1-\frac{\left\langle\phi^{4}\right\rangle}{3\left\langle\phi^{2}\right\rangle^{2}}
$$

Si además, consideramos [Binder, 1981] el comportamiento de los momentos $\left\langle\phi^{k}\right\rangle_{\mathrm{L}}$ en términos de la longitud de correlación $\xi$, cerca del punto crítico, tenemos:

$$
\begin{array}{r}
\left\langle\phi^{k}\right\rangle_{L}=L^{-k y}\left(a^{k} C_{0}\right)^{-1} f_{k}(\xi / L) \\
U=1-\frac{C_{0} f_{4}(\xi / L)}{3 f_{2}^{2}(\xi / L)} \rightarrow U^{*}=1-\frac{C_{0} f_{4}(\infty)}{3 f_{2}^{2}(\infty)}
\end{array}
$$

Donde $y, a$ y $\mathrm{C}_{0}$ son cantidades independientes de L y constantes. Si nos posicionamos en el punto critico, $\mathrm{U}$ alcanza un valor $\mathrm{U}^{*}$ independiente de $\mathrm{L}$. Para distintos valores de L suficientemente grandes, las curvas U se cruzan en función de la temperatura (o alguna otra cantidad relevante al sistema) en un punto fijo $\mathrm{U}^{*}$. La ubicación del punto fijo de cruce es el punto crítico. Esto hace que el calculo del cumulante de Binder para sistemas de distinto L sea una excelente herramienta para hallar la presencia y ubicación del punto crítico.

Con esto en mente, en vez de realizar simulaciones para distintos tamaños de sistema L, podemos utilizar una única simulación para un sistema con L grande, para realizar un estudio de tamaño finito dividiendo al sistema en sub-bloques [Landau and Binder, 2014] [Binder, 1981] como describimos en la próxima sección.

\subsubsection{El Análisis de Sub-Bloques como Medida de Efectos de Tamaño Finito}

En su trabajo [Trefz et al., 2017], Trefz y colaboradores estudian un sistema de partículas propulsadas con una interacción orientacional. El sistema presenta una separación de fase, entre una fase rica en coloides y otra rica en polímeros, donde el diagrama de fase depende fuertemente de las interacciones orientacionales. Para realizar una determinación del punto crítico y una estimación de exponentes críticos, los autores utilizan un análisis de sub-bloques.

El análisis de sub-bloques [Rovere et al., 1990, Binder, 1981] consiste en tomar a un sistema de volumen $\mathrm{V}$, con una dinámica canónica y dividirlo en $n_{B}$ volúmenes idénticos mas pequeños que no se solapan, los cuales llamaremos bloques. Naturalmente el lado B del bloque debe satisfacer $B \leqslant L$ de manera que el volumen $V=L^{d}$ quede completamente cubierto. Si bien el análisis vale para cualquier dimensión, nos enfocaremos en $\mathrm{d}=2$. 
De esta manera, el número de partículas en los bloques puede fluctuar, simulando así un sistema quasi gran canónico. Luego, para cada bloque podemos estudiar la densidad local y compararla con la global (la cual esta fija) determinando los momentos:

$$
\begin{aligned}
& m^{2}=\frac{1}{n_{B}^{3}} \sum_{i}\left(\rho_{i}-\rho\right)^{2} \\
& m^{4}=\frac{1}{n_{B}^{3}} \sum_{i}\left(\rho_{i}-\rho\right)^{4}
\end{aligned}
$$

Donde $\rho=\frac{\mathrm{N}}{\mathrm{L}^{2}}$ es la densidad global (constante) y $\rho_{i}=\frac{\mathrm{N}_{i}}{\mathrm{~B}^{2}}$ es la densidad local, para un bloque de volumen $\mathrm{B}^{2}$ que contiene $\mathrm{N}_{i}$ partículas. El número de bloques viene dado por $n_{B}=L / B$. Notamos que $B$ debe ser un múltiplo entero de $L$ de manera que $n_{B}$ sea entero. Exploraremos una alternativa en la sección 7.4.3

La utilidad del análisis de bloques reside en que solo es necesario realizar simulaciones para un único tamaño de sistema L suficientemente grande. Luego, contando con las coordenadas de las partículas, podemos realizar a posteriori un estudio de tamaño finito variando el tamaño del lado de los bloques B.

\subsubsection{Muestreo Utilizando Bloques}

La elección del tamaño del bloque B debe ser cuidadosa. Un valor de B muy pequeño implica que las fluctuaciones por el agregado o remoción de una partícula sean muy grandes. Un valor $\mathrm{B} \simeq \mathrm{L}$ implicaría $n_{\mathrm{B}}$ muy pequeño, lo que volvería la correlación entre bloques muy grande.

Podemos pensar que $\mathrm{B}$ debe satisfacer $\xi \ll \mathrm{B} \ll \mathrm{L}$, donde $\xi$ es la distancia de correlación, la cual es constante pero incógnita. Con estos cuidados, seleccionamos distintos valores para B de manera arbitraria.

En este trabajo de tesis, como alternativa a tomar B tal que sean múltiplos enteros de $\mathrm{L}$, optamos tomar $\mathrm{B} \ll \mathrm{L}$ donde $\mathrm{B}$ es próximo a un múltiplo entero de $\mathrm{L}$. Como muestra la figura 7.2, esto implica que no es posible cubrir el espacio completamente con volúmenes $\mathrm{B}^{2}$.

Notemos que si B no es múltiplo entero de $L$, entonces $n_{B}=L / B$ resulta un numero real. Si llamamos $n_{B}^{\prime}$ a la parte entera de $n_{B}$, podemos cubrir un volumen $V^{\prime}<V=L^{2}$ con $n_{B}^{\prime}$ bloques.

Sin embargo, podemos usar el espacio no cubierto como una herramienta más del muestreo. Si cada vez que realizamos una medida con el análisis de sub-bloques, colocamos al volumen $V^{\prime}$ en una posición aleatoria dentro de $V$, podemos repetir las medidas para distintas posiciones y finalmente seleccionar la que presenta mayor varianza para 

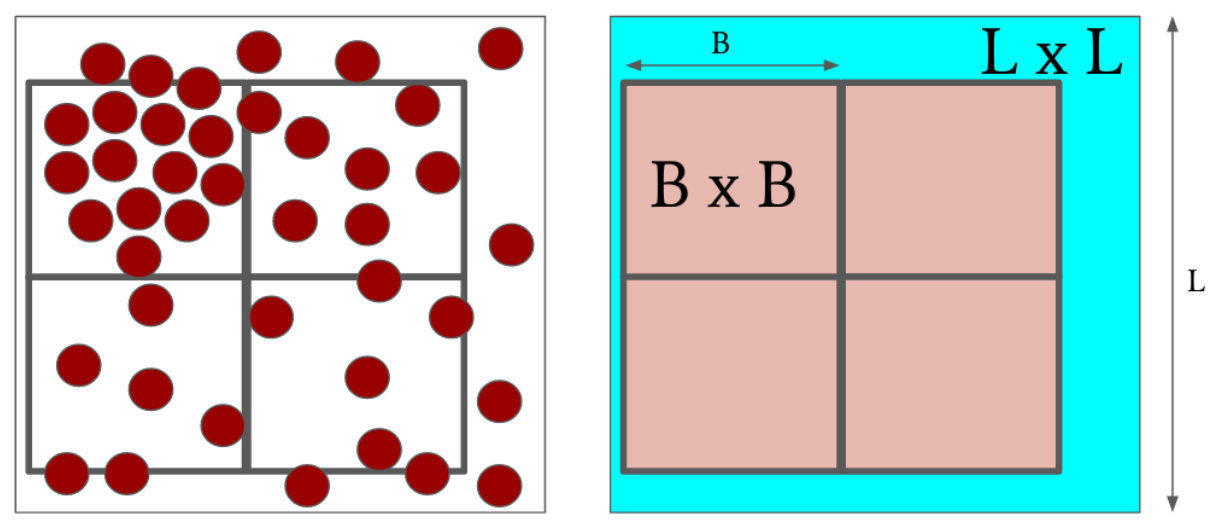

Figura 7.2: Representación del análisis de sub-bloques, usando bloques de lado B que no son múltiplos enteros de L.

calcular las cantidades locales.

Una manera sencilla de realizar esta variación, que también resulta útil para el caso en que los bloques cubren perfectamente el volumen, es la de realizar un único desplazamiento rígido aleatorio de las partículas, antes de realizar el análisis de sub-bloques. Valiéndonos de las condiciones de contorno periódicas, es posible analizar la varianza entre bloques para distintos desplazamientos $(\delta x, \delta y)$ y seleccionar la que genera mayor varianza, pues eso implica que sera el arreglo que capture mayor diversidad de distribuciones locales de partículas. Mencionamos que esta elección no presenta un sesgo para las medidas, pero sí disminuye el ruido presente en las curvas para las que el retículo esta ubicado en una posición fija, cubriendo todo el volumen. De esta manera, en caso de cambiar el numero de partículas $\mathrm{N}$ y tamaño de caja L, para densidad constante, los mismos tamaños de bloque B pueden ser utilizados para un nuevo estudio, lo que permite una comparación directa entre sistemas de distinto L. 


\section{Capítulo 8}

\section{Resultados para Partículas Auto-Propulsadas con Volumen Excluído}

\subsection{El modelo}

En este capítulo estudiamos una mezcla binaria de partículas autopropulsadas. Todas las partículas poseen el mismo volumen excluido y experimentan la misma intensidad $\epsilon$ de fuerza Lennard Jones, mediante un potencial Lennard Jones truncado en su mínimo como el descripto en la sección 7.1. La única diferencia entre las dos tipos de partículas reside en su velocidad, cada tipo de partícula tiene una velocidad constante en módulo. De esta manera, podremos clasificar a las partículas en lentas y rápidas. Mencionamos también que las partículas viven en una caja cuadrada bidimensional de volumen $\mathrm{V}=\mathrm{L}^{2}$, con condiciones de contorno periódicas.

Dada la restricción de que la rapidez de las partículas es constante, notamos que el efecto de las interacciones es únicamente el de cambiar la dirección del movimiento, dejando su rapidez constante. Para realizar una integración de las ecuaciones de movimiento, utilizamos una implementación tipo Euler, usando el siguiente algoritmo de calculo: 


$$
\begin{array}{r}
\vec{x}(t+d t)=\vec{x}(t)+\vec{v}(t) d t \\
\overrightarrow{v^{\prime}}=\vec{v}(t)+\vec{F}(t) d t \\
\theta=\operatorname{atan} 2\left(v_{y}^{\prime}, v_{x}^{\prime}\right) \\
\vec{v}(t+d t)=v_{0}^{\top}[\cos \theta \hat{i}+\sin \theta \hat{j}]
\end{array}
$$

Usamos la función atan2 para extraer el ángulo que forma la velocidad. Esta función computa el valor principal de la función argumento aplicada al número complejo a $+i b$. Es decir, $\operatorname{atan} 2(b, a)=\operatorname{Pr}[\arg (a+i b)]=\operatorname{Arg}(a+i b)$. De esta manera un cambio de argumento en $2 \pi$ no tiene efecto y la función queda definida (usando el valor principal) en el rango $(-\pi, \pi]$ resultando: $-\pi<\operatorname{atan} 2(b, a) \leqslant \pi$. $v_{0}^{\top}$ representa la rapidez constante para el tipo de partícula $\mathrm{T}$. La fuerza $\mathrm{F}^{j}$ es la de Lennard Jones:

$$
\overrightarrow{F^{j}}=\sum_{i} 24 \epsilon \sigma^{6} r_{i j}^{-8}\left[2 \sigma^{6} r_{i j}^{-6}-1\right] \vec{r}_{i j}
$$

Donde $i$ recorre las i-esimas partículas a una distancia $r_{i j}$ de la partícula $j$ con $r_{i j} \leqslant$ $2^{1 / 6} \sigma$. Notamos que la fuerza no esta acotada, pero esta integración de las ecuaciones de movimiento evita que las partículas experimenten el comportamiento explosivo causado por proximidad elevada, mencionado en el capitulo 7 .

\subsection{El Espacio de Parámetros Utilizado}

Puesto que son las colisiones las que controlan la dinámica del sistema, utilizaremos como observables a los choques entre partículas, donde definimos choque o colisión, como dos partículas que se aproximan a una distancia $r_{i j} \leqslant 2^{1 / 6} \sigma$.

Como tenemos interés en los efectos de separación de fase, el observable central será la cantidad relativa de colisiones entre una partícula lenta (S) y una partícula rápida $(\mathrm{F})$. Sea $\mathrm{n}_{\mathrm{SF}}$ el número de colisiones en un dado instante entre partículas de distinto tipo y $\mathrm{N}$ el número total de partículas, el observable que mide las colisiones debidas a la interacción Lennard Jones es denotado LJSF y viene dado por:

$$
\mathrm{LJSF}=\frac{\mathrm{n}_{\mathrm{SF}}}{\mathrm{N}}
$$

Con esta definición, LJSF disminuye su valor a medida que el sistema experimenta una 
segregación. Elegimos utilizar el modulo de la velocidad de las partículas lentas, $\mathrm{V}_{\mathrm{S}}$, como parámetro de orden. Por ello, decidimos fijar el modulo de la velocidad de las partículas rápidas $V_{F}$, con $V_{S} \leqslant V_{F}=0.1$. Fijando el paso temporal en $d t=0.002$, como se menciono en la sección 7 , permite despreocuparnos de pasar por alto efectos sutiles que se perderían utilizando desplazamientos abruptos (ya sea por un dt y/ó $V_{\mathrm{F}}$ mayor al seleccionado).

Parte de nuestros objetivos fue utilizar el esquema de sub-bloques para realizar estudios de tamaño finito. Para ello utilizamos sistemas con numero total de partículas $N=1000$ con un tamaño de caja $L=45$, lo que representa una densidad $\rho \simeq 0.49$. La proporción de partículas, esta controlada por la fracción de partículas rápidas $0 \leqslant$ FPR $\leqslant 1$, donde $N=N_{\text {Rapidas }}+N_{\text {Lentas }}=N F P R+N(1-F P R)$. Salvo se mencione lo contrario, utilizamos FPR $=0.5$ para los estudios realizados.

Por simplicidad, mantuvimos los parámetros de la fuerza de Lennard Jones en $\epsilon=1$ y $\sigma=1$ para ambos tipos de partícula. Recalcamos que la única diferencia entre las partículas o su interacción, es la que viene dada por su rapidez.

\subsection{Determinación de comportamiento estacionario}

El sistema se encuentra manifiestamente fuera del equilibrio. Para estudiarlo, buscamos determinar que el sistema alcanza estados estacionarios. Si asumimos que son las colisiones las que regulan el comportamiento, verificamos que el sistema estaciona cuando el observable LJSF estaciona. Sin embargo, esta determinación resulta problemática cuando $\mathrm{V}_{\mathrm{S}}<<\mathrm{V}_{\mathrm{F}}$, pues la dinámica se enlentece y no resulta claro, de la evolución de LJSF, si el sistema ha estacionado o si continua evolucionando lentamente. Es por esto, que así como en el estudio de sistemas vítreos, el enlentecimiento de la dinámica llevó a que recurramos a métodos complementarios para verificar estacionareidad. Inspirándonos en la prueba BIC utilizada en la sección 3.5.2, realizamos una prueba de condición inicial para verificar estacionareidad.

Dicha prueba consistió en realizar simulaciones para un mismo conjunto de parámetros, partiendo tanto desde una configuración inicial de posiciones aleatorias, así como de una configuración artificialmente segregada de partículas lentas distribuidas en forma de circulo, como muestra la figura 8.1. La configuración artificialmente segregada, ubica a las partículas en un arreglo periódico, donde la densidad del circulo es $\rho=2$. Dado que la dirección de la velocidad inicial es aleatoria para todas las configuraciones, es posible generar tantas realizaciones distintas como se deseen. Optamos por una geometría circular, pues se asemeja a la que el sistema adopta cuando $V_{S}<0.01$. Además, 

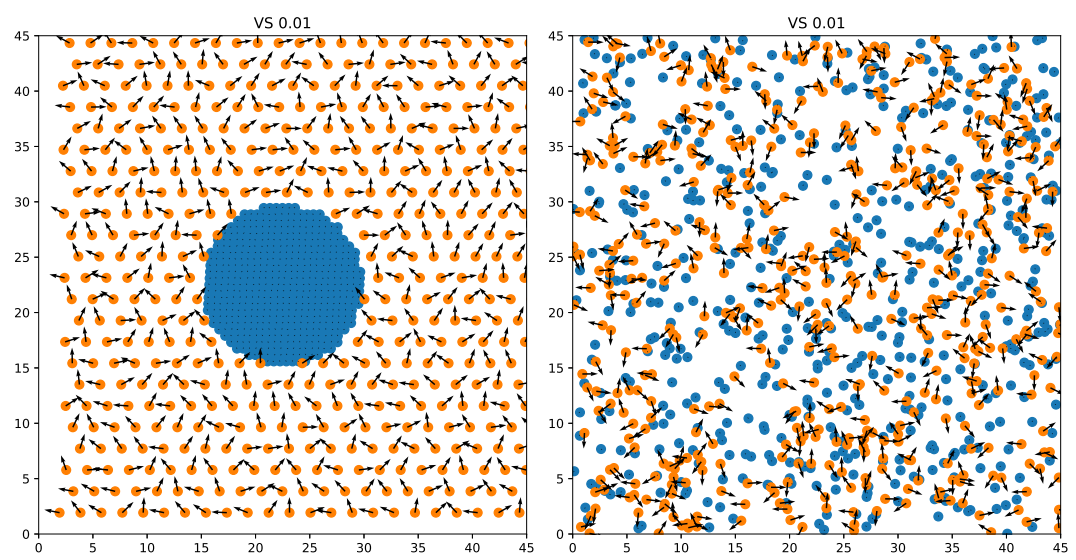

Figura 8.1: Presentamos configuraciones iniciales con el mismo conjunto de parámetros. A la izquierda, presentamos una bola de partículas lentas confinada por las partículas rápidas. A la derecha partículas sorteadas en posiciones aleatorias. En este caso, ambas representan sistemas con $V_{S}=0.01$.

partiendo desde una configuración completamente segregada, el valor de LJSF es mínimo, por lo que esperamos que este observable crezca al evolucionar el sistema. En las figuras 8.3 y 8.2 damos una muestra de la ergodicidad del sistema, mostrando que sin importar la configuración inicial, un dado conjunto de parámetros posee un valor estacionario bien definido.

Este método presenta el beneficio adicional, de acortar fuertemente los tiempos de simulación, pues determinamos que el sistema ha estacionado luego de que ambas curvas se encuentran. Como muestra la figura Fig. 8.3, para $V_{S}=0.008$, partir desde una configuración inicial con un arreglo circular de partículas lentas, resulta $10^{3}$ mas rápido en alcanzar el comportamiento estacionario que comenzando desde el desorden. Por otra parte, $\mathrm{V}_{\mathrm{S}}=0.02$ demora aproximadamente el mismo numero de pasos de simulación en llegar al régimen estacionario, partiendo tanto desde el desorden como de un arreglo circular. La detección de esta diferencia, nos permite optimizar las simulaciones realizadas de acuerdo al valor de $\mathrm{V}_{\mathrm{S}}$ utilizado, eligiendo de manera astuta el arreglo de la configuración inicial tal que llegue al valor estacionario mas aprisa. 

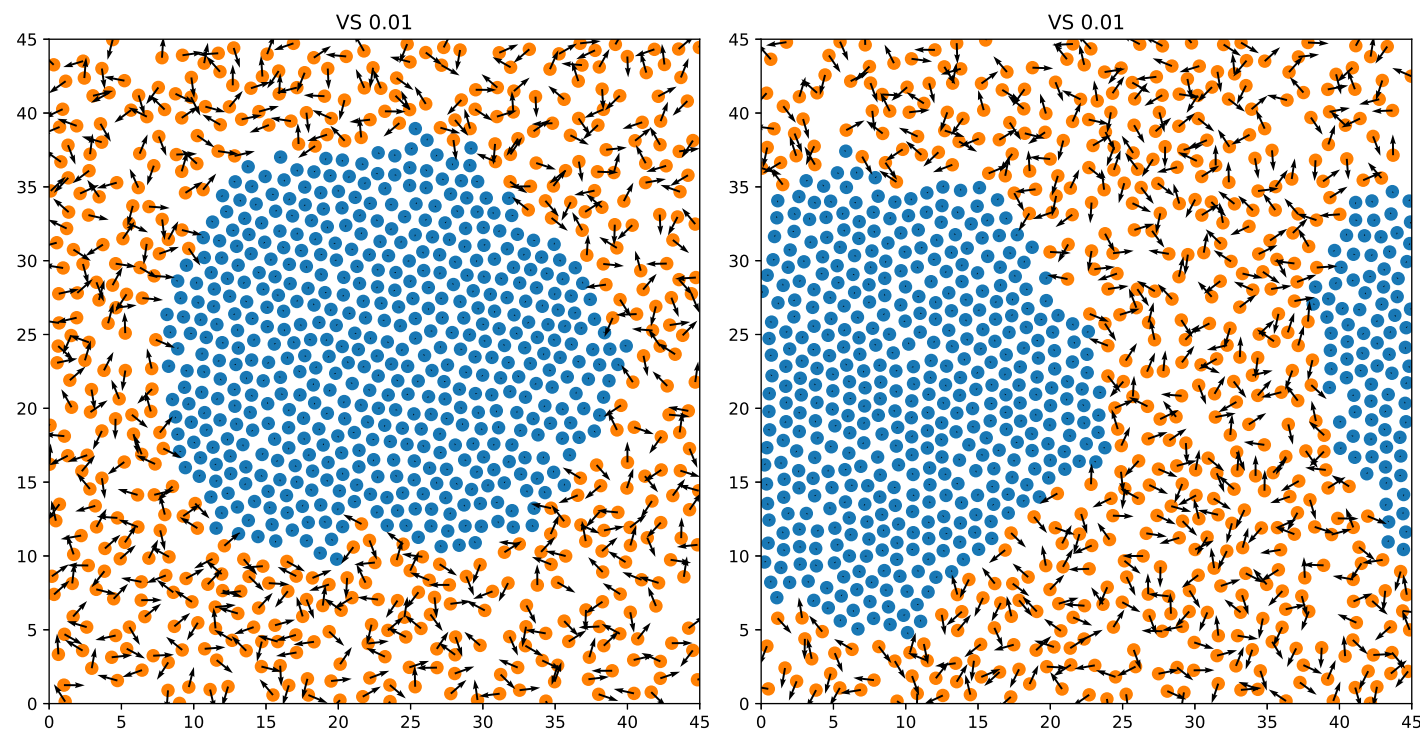

Figura 8.2: Presentamos configuraciones finales con el mismo conjunto de parámetros y las configuraciones iniciales presentadas en la figura 8.1. Observamos que la hipótesis de ergodicidad vale para estos escenarios, alcanzando sistemas con el mismo comportamiento partiendo desde situaciones iniciales opuestas.

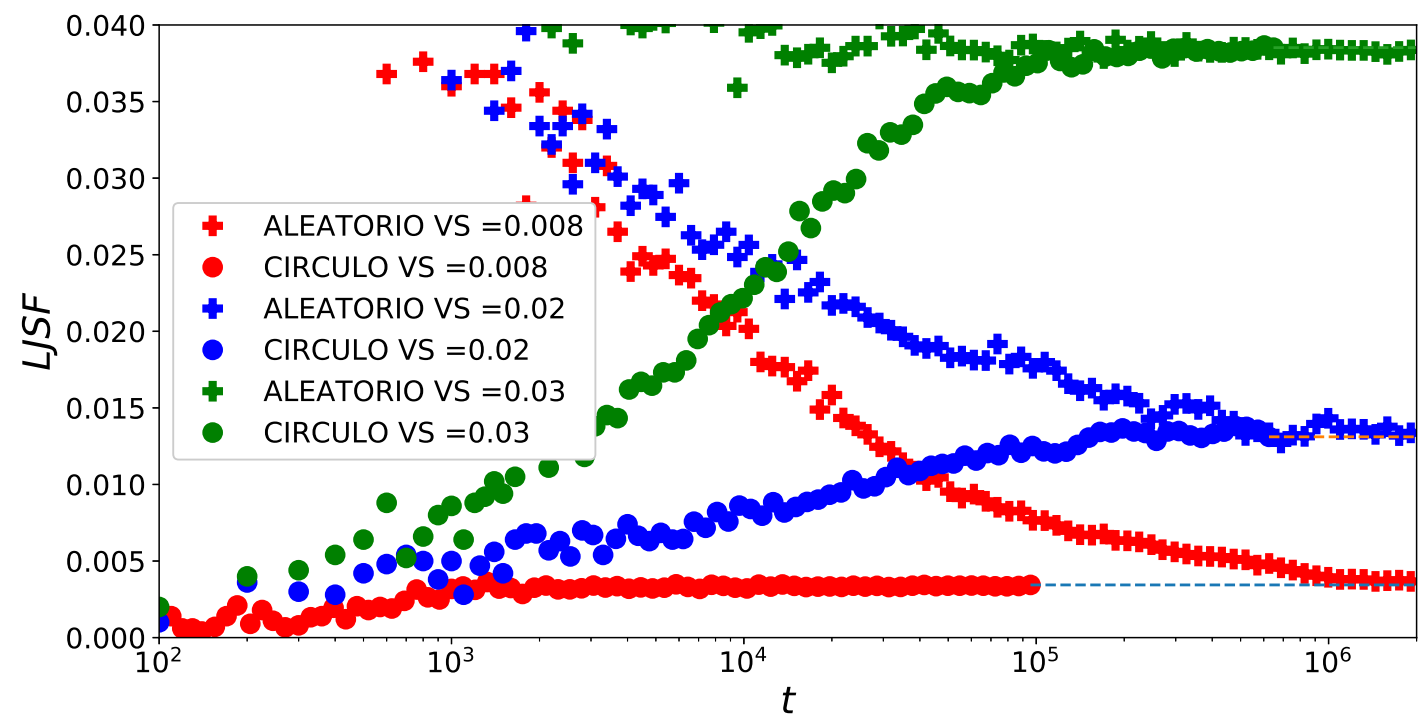

Figura 8.3: Utilizando los mismos parámetros, pero partiendo desde configuraciones iniciales distintas, podemos encontrar mediante una variante de la prueba BIC, el régimen estacionario del sistema. Observamos que la elección de la configuración inicial (segregada o mezcla homogénea), influye en el tiempo en que el sistema demora en alcanzar el valor estacionario. 


\subsection{Clasificación de Estados Estacionarios Según $V_{S}$}

Utilizando la metodología presentada en las sección previa, podemos explorar el espacio de fase presentado en la tabla. 8.1 variando únicamente $\mathrm{V}_{\mathrm{S}}$.

Presentamos en la figura. 8.4 algunos de los estados estacionarios del sistema para distintos valores de $V_{S}$. Para los que el criterio de estado estacionario es la estacionareidad de LJSF.

Una vez que el sistema alcanza el régimen estacionario, utilizamos la configuración final, como punto de partida para una simulación posterior. Llamamos medidas estacionarias, a las simulaciones que toman como punto de partida al sistema estacionado. Será mediante las medidas estacionarias que estudiaremos la segregación del sistema en función de $V_{S}$.

Además, observamos que la intensidad de la segregación, medida desde LJSF asintótico, depende fuertemente de la densidad $\rho$. Para ello, definimos $\mathrm{LJSF}_{\infty}=\langle\overline{\mathrm{LJSF}(\mathrm{t})}\rangle$ para medidas estacionarias, donde $\langle\ldots\rangle$ representa el promedio entre realizaciones y $\overline{(\ldots)}$ representa el promedio temporal. Si dejamos fijo el numero de partículas $\mathrm{N}$ y aumentamos el tamaño de la caja L, como muestra la Figura 8.5, observamos que la dependencia de $\mathrm{LJSF}_{\infty}$ con $\mathrm{V}_{\mathrm{S}}$ decae, tanto que para $\mathrm{L}=90$ todos los valores de $\mathrm{V}_{\mathrm{S}}$ poseen valores de $\mathrm{LJSF}_{\infty}$ prácticamente iguales.

La Figura 8.4 muestra estados mezclados y estado segregados, lo que sugiere la existencia de una transición de segregación en algún valor intermedio de $\mathrm{V}_{\mathrm{s}}$. A continuación, estudiamos la naturaleza y punto de la transición mediante otras técnicas.

\begin{tabular}{|c|c|c|c|c|c|c|}
\hline FPR & $\mathrm{V}_{\mathrm{F}}$ & $\epsilon$ & $\mathrm{L}$ & $\mathrm{N}_{\text {Particulas }}$ & $\mathrm{dt}$ & Realizaciones \\
0.5 & 0.1 & 1 & 45 & 1000 & 0.002 & 10 \\
\hline
\end{tabular}

Cuadro 8.1: Valores constantes en la simulación (a menos se mencione explícitamente lo contrario). Las simulaciones para alcanzar el régimen estacionario, tienen un tiempo $5 \times 10^{5} \leqslant t \leqslant 2 \times 10^{6}$, mientras que todas las de medidas estacionarias $t=10^{5}$. 

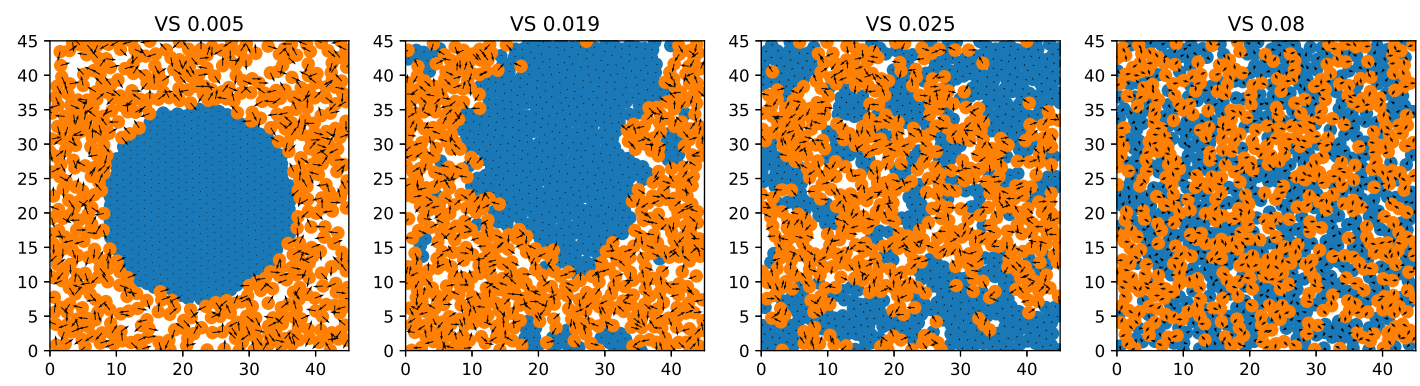

Figura 8.4: Variando $V_{S}$, estudiamos las configuraciones finales. Notamos que el sistema presenta segregación e incluso parece comenzar a formar una estructura periódica de mayor densidad cuando $V_{S} \leqslant 0.01$.

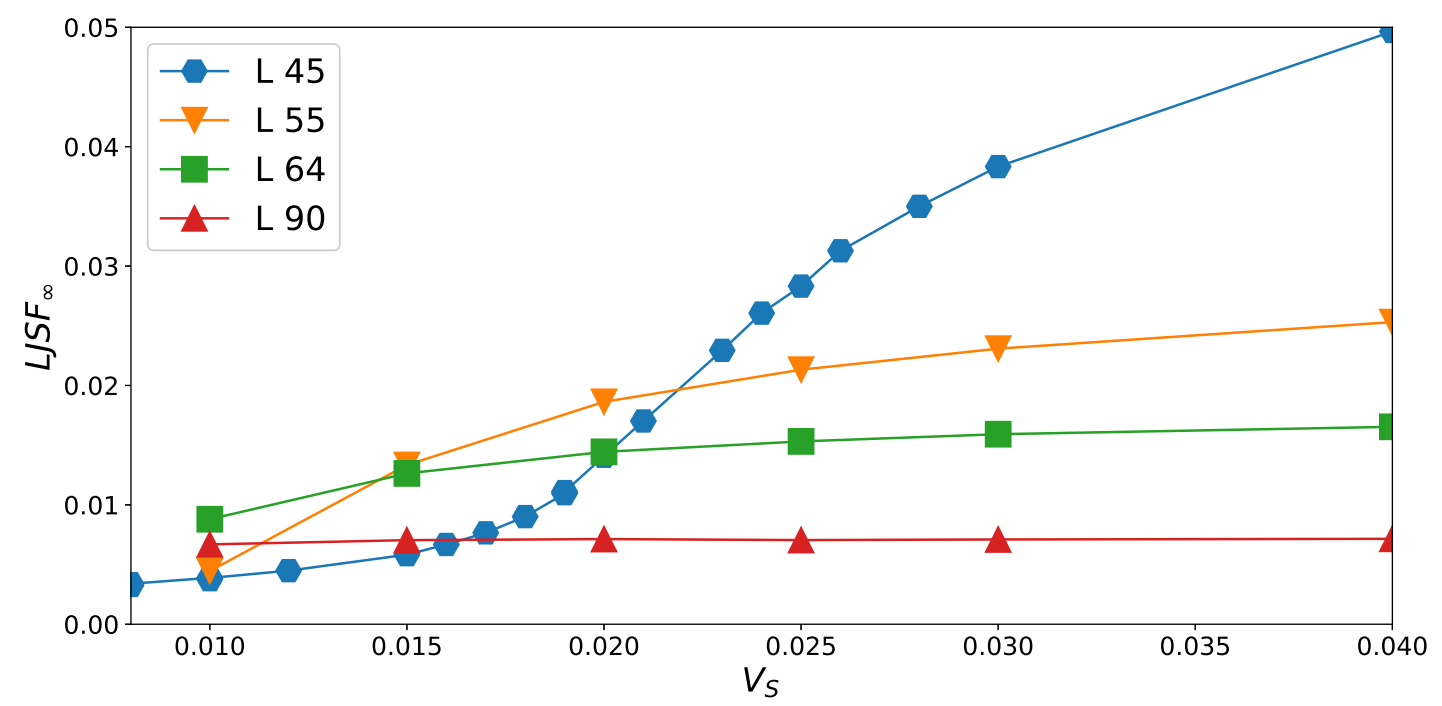

Figura 8.5: A partir de medidas estacionarias de $\mathrm{LJSF}_{\infty}$ para cajas de tamaño $\mathrm{L}=$ $45,55,64,90$ y $\mathrm{N}$ fijo, observamos que la dependencia de $V_{S}$ va disminuyendo a medida que la densidad decrece. 


\subsection{Distribuciones Locales de Densidad y Concentración}

Utilizando el esquema de sub-bloques presentado en la sección 7.4.3, fuimos capaces de detectar los comportamientos de la densidad local $\rho_{\mathrm{B}}$ y concentración local $\mathrm{C}_{\mathrm{B}}$. Para un bloque de lado $\mathrm{B}$, con $\mathrm{N}_{\mathrm{B}}$ partículas en su interior, la densidad local está dada por $\rho_{\mathrm{B}}=\mathrm{N}_{\mathrm{B}} / \mathrm{B}^{2}$, mientras que la concentración molar de partículas rápidas, viene dada

por $C_{B}^{F}=\frac{N_{B}^{F}}{N_{B}^{F}+N_{B}^{S}}$. Notamos que sus contrapartes globales, $\rho$ y $C^{F}=F P R$, permanecen constantes en todo momento.

En su trabajo [Rovere et al., 1990], Rovere y colaboradores estudian un fluido bidimensional con un potencial de Lennard-Jones truncado. Allí, los autores encuentran una estructura de dos picos para la función de densidad por bloques, permitiendoles estimar la densidad de las 2 fases que surgen cuando el sistema se segrega. Utilizando la estructura de sub-bloques que presentamos en la sección 7.4.3, calculamos funciones de densidad de bloque cuya estructura representa la distribución de densidades locales del sistema. Realizamos también un análisis análogo para la concentración local.

Estudiamos los efectos de tamaño finito utilizando bloques de tamaño B, donde $B=[11.25,9 ., 7.5,6.42,5.625,5.0,4.5,3.75,3.2,2.8125]$. En la Figura 8.6 presentamos los resultados del estudio de la densidad de bloques de tamaño $B=5.0$ en comparación con la densidad global del sistema $\rho=\frac{\mathrm{N}}{\mathrm{L}^{2}} \simeq 0.49$. El histograma en dicha figura, representa tanto a los estados mixtos, donde la densidad de los bloques coincide mayormente con la densidad del sistema global, así como al caso segregado, donde existen 2 regiones con densidades locales distintas a la densidad global. Los cambios de concavidad de dicho histograma, nos dan un indicio de la región donde podemos encontrar valores de transición mezcla-segregación. Notamos que el análisis estudia el valor relativo $\rho_{B} / \rho$, de manera que el valor $1 \rightarrow \rho_{\mathrm{B}}=\rho$. Es decir, que valores que se aparten de 1 , representan bloques cuya distribución de partículas posee una densidad $\rho_{\mathrm{B}}$ mayor o menor a la $\rho$ global.

Sobre los resultados presentados en la Figura 8.6, podemos realizar una determinación de $\rho_{\mathrm{B}}\left(\mathrm{V}_{\mathrm{S}}\right)$ mediante el ajuste de funciones gaussianas alrededor de la posición de los máximos, como presentamos en la Figura 8.7. De esta manera, cuando el sistema se segrega, podemos extraer un valor $\rho_{\min }$ para la región de baja densidad y un valor $\rho_{\max }$ para la región de alta densidad. Usando estos valores, determinamos el comportamiento de la diferencia de densidades de las dos sub-regiones segregadas como una ley de potencia con exponente $\zeta$, dada por $\Delta \rho_{\mathrm{B}}=\rho_{\max }\left(\mathrm{V}_{\mathrm{S}}\right)-\rho_{\min }\left(\mathrm{V}_{\mathrm{S}}\right)=\mathrm{V}_{\mathrm{S}}^{\zeta}$. Mostramos este resultado en la Fig. 8.8

Respecto a la concentración local de partículas rapidas $\mathrm{C}_{\mathrm{B}}^{\mathrm{F}}$ (Figura 8.9), observa- 


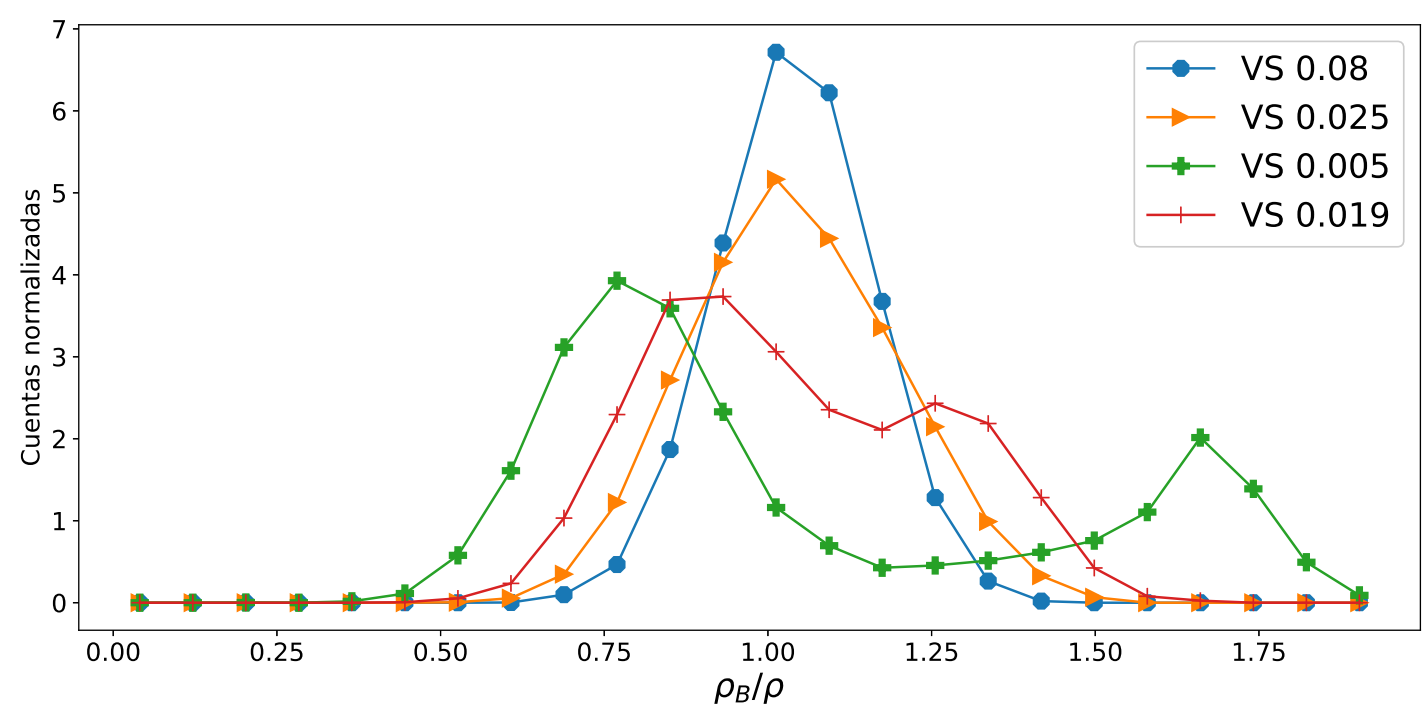

Figura 8.6: Usando el esquema de sub-bloques, con bloques de tamaño $B=5.0$, estudiamos la distribución de densidades de los bloques en función de la densidad global, para $0.005 \leqslant V_{S} \leqslant 0.09$. Observamos dos tipos de comportamiento: un régimen de mezcla, donde la distribución coincide con el valor de la densidad global, así como otro para los cuales el sistema esta segregado en dos regiones de densidades locales muy distintas. $V_{S} \leqslant 0.2$ representa la región segregada y $V_{S}>0.2$ representa la región mezclada.

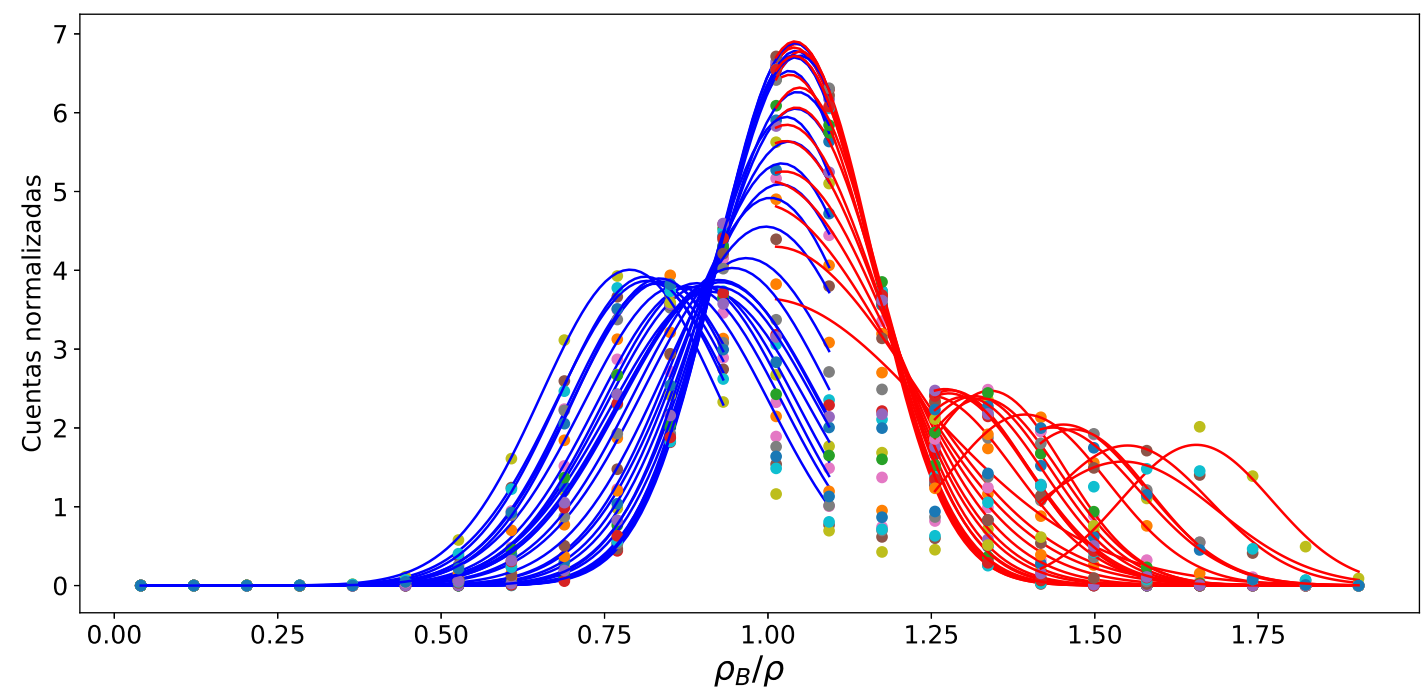

Figura 8.7: Utilizamos los resultados de la Fig. 8.6 para realizar la determinación de las densidades principales para cada $\mathrm{V}_{\mathrm{S}}$, mediante un ajuste gaussiano para cada distribución. 


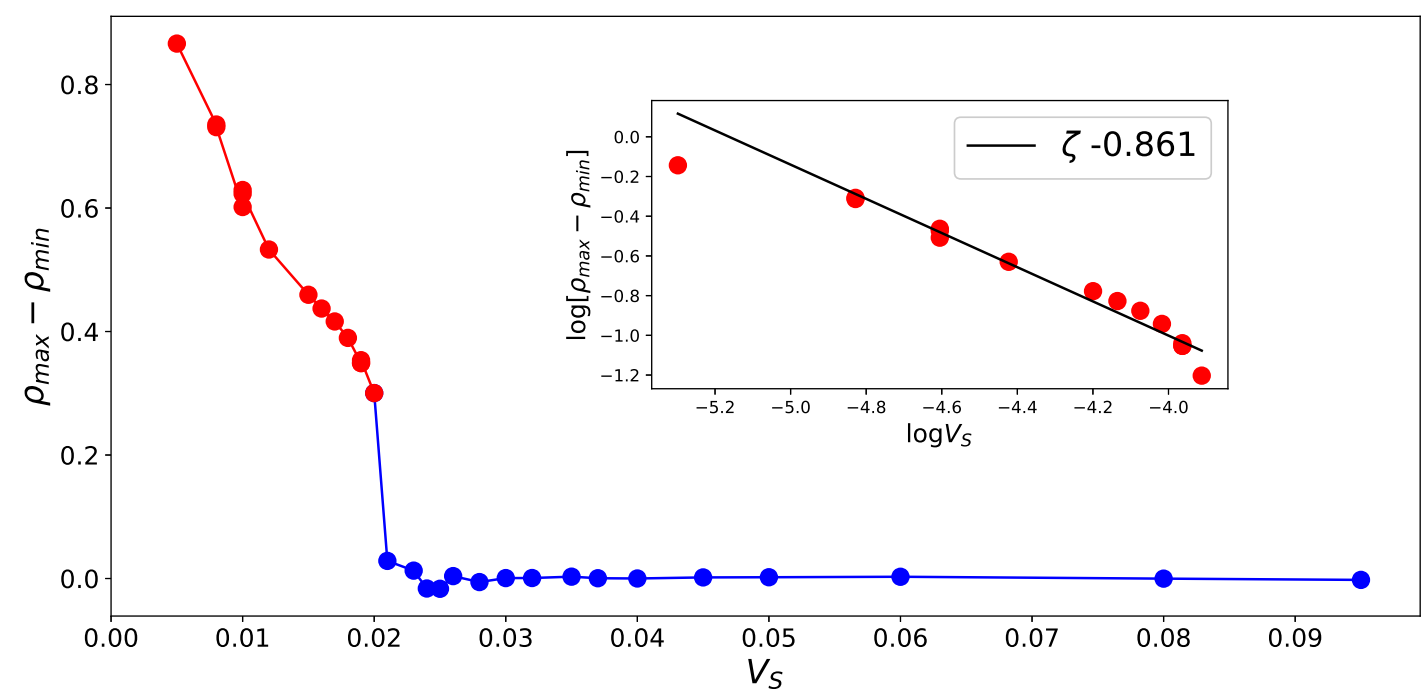

Figura 8.8: Usando las densidades determinadas en la figura 8.7, podemos estudiar su diferencia en función de $V_{S}$. De esta manera, podemos extraer un comportamiento de ley de potencia, con un exponente $\zeta \simeq-0.861$

mos nuevamente el mismo comportamiento que para la densidad. Podemos detectar dos comportamientos en función de $\mathrm{V}_{\mathrm{S}}$ : Un caso no-segregado donde la concentración local coincide mayormente con la concentración global y un caso segregado donde la concentración local tiende a valores 0 o 1 (según el caso estudiado).

Los cambios de la concavidad en la distribución de concentración, a medida que $\mathrm{V}_{\mathrm{S}}$ disminuye, permiten determinar que el sistema se redistribuye de regiones con concentraciones locales $\mathrm{C}_{\mathrm{B}}^{\mathrm{F}} \simeq \mathrm{C}^{\mathrm{F}}=\mathrm{FPR}$ a regiones que tienden a $\mathrm{C}_{\mathrm{B}}^{\mathrm{F}}=0$ ó $\mathrm{C}_{\mathrm{B}}^{\mathrm{F}}=1$, indicando segregación.

\subsection{Transición de segregación y cumulantes de Binder}

Utilizando la información extraída de las medidas estacionarias mediante el análisis de sub-bloques, podemos realizar el cálculo del cumulante de Binder (introducido en la sección 7.4.1) de distintas cantidades. En la Figura 8.10 mostramos el cálculo del cumulante para la concentración de las partículas rápidas $\mathrm{U}\left(\mathrm{C}^{f}\right)$. Observamos que presenta un punto de cruce para todos los tamaños de sub-bloque B estudiados. Esto señala una transición de fase de segundo orden para un valor de $V_{S} \simeq 0.0195$, el cual coincide con las observaciones que extrajimos mediante los estudios de densidad local $\rho_{\mathrm{B}}$ y concentración local $\mathrm{C}_{\mathrm{B}}^{\mathrm{F}}$.

Para entender si el fenómeno se mantiene presente para distintas concentraciones globales (FPR), podemos repetir el calculo del cumulante $\mathrm{U}\left(\mathrm{C}^{\mathrm{F}}\right)$ para distintos valores 


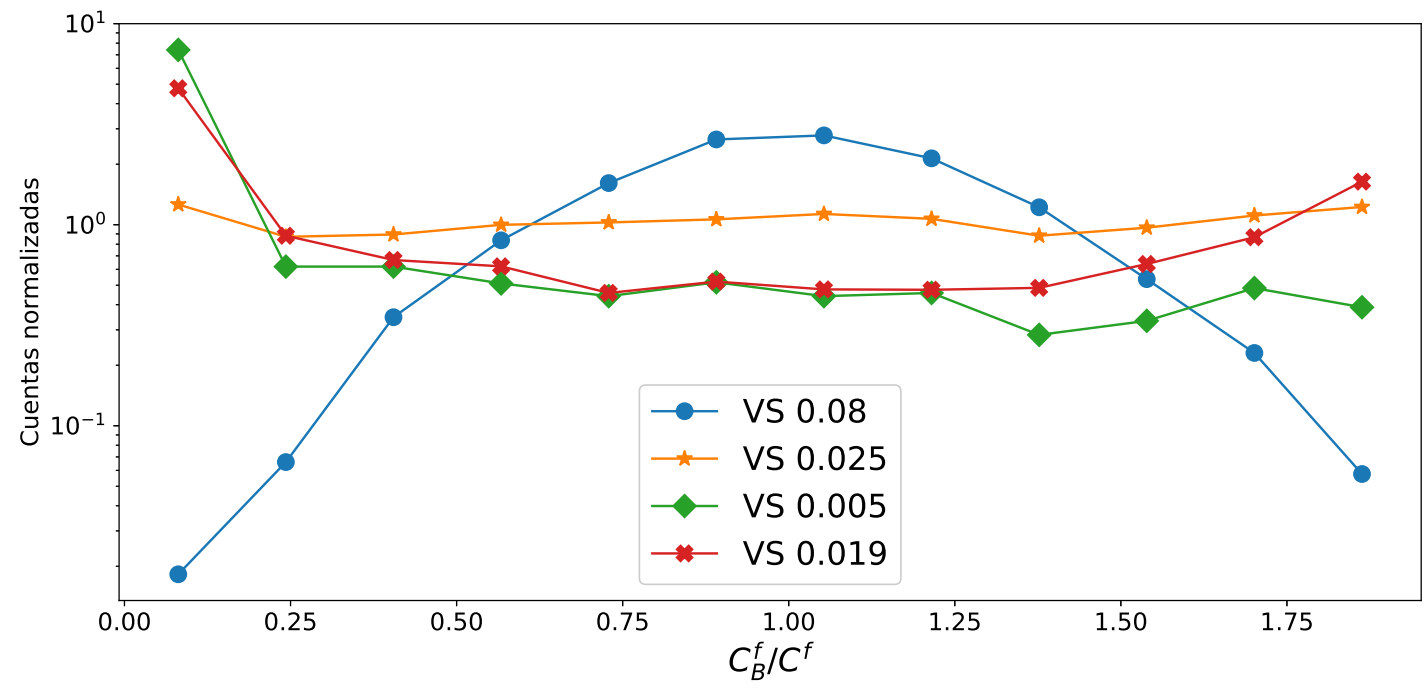

Figura 8.9: Estudiando la distribución de concentración de partículas rápidas $C^{F}$ al variar $V_{S}$ volvemos a determinar regiones de comportamiento segregado $\left(V_{S}<0.02\right)$, así como regiones de mezcla de partículas $\left(V_{S}>0.02\right)$.

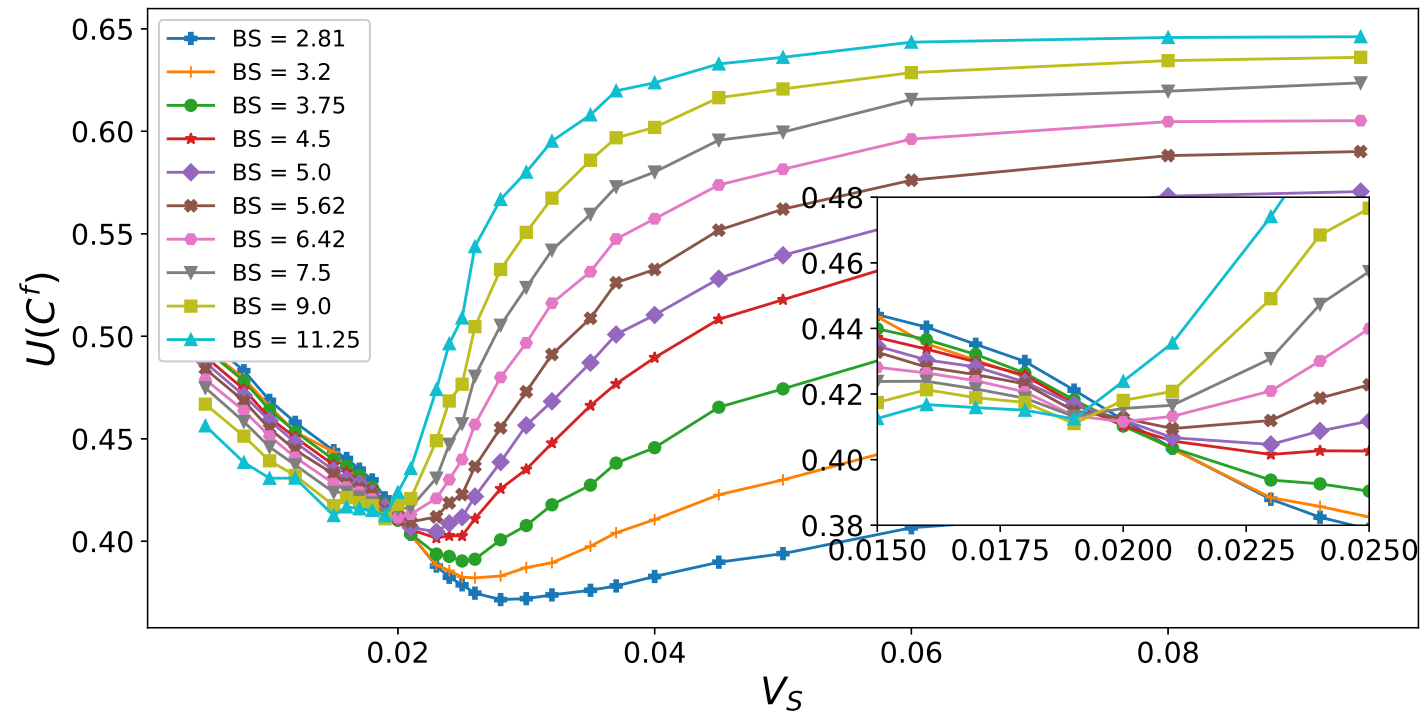

Figura 8.10: Calculo del cumulante de Binder para concentración de partículas rápidas $\mathrm{U}\left(\mathrm{C}^{\mathrm{f}}\right)$, en función de $\mathrm{V}_{\mathrm{S}}$ para $\mathrm{FPR}=0.5$. El cruce de las curvas de distintos tamaño de sub-bloque señala una transición de fase de segundo orden en $V_{S} \simeq 0.0195$. 


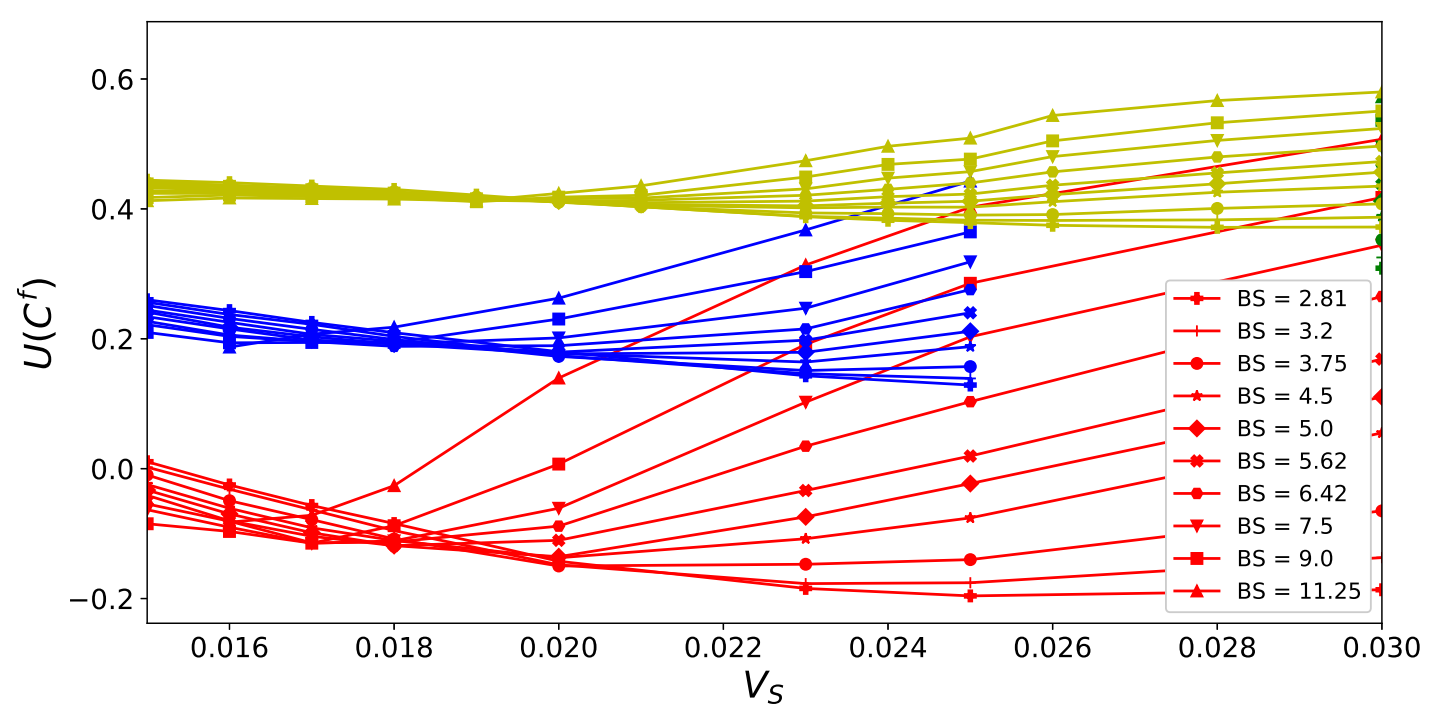

Figura 8.11: Calculo del cumulante de Binder para concentración de partículas rápidas $\mathrm{U}\left(\mathrm{C}^{\mathrm{f}}\right)$, en función de $\mathrm{V}_{\mathrm{S}}$ para distintos valores de FPR. Donde verde representa $F P R=0.5$, azul representa $F P R=0.35$ y rojo representa $F P R=0.25$. Observamos que a medida que FPR disminuye, el cruce de curvas se deslocaliza.

de FPR, manteniendo N y L constante. Presentamos en la figura 8.11 la comparación de los cumulantes para $F P R=0.5,0.35,0.25$. Observamos que existe una deslocalización del punto de cruce, a medida que disminuye FPR. Esto sugiere que se desvanece la transición de segundo orden que fue detectada para FPR $=0.5$ al disminuir de ese valor.

\subsection{Detección de Orden Mediante $S(k)$ y $g(r)$}

Para detectar la presencia de orden espacial en función de $V_{S}$, realizamos un estudio de correlaciones espaciales usando las funciones $g(r)$ y $S(k)$ (introducidas en la sección 2.4.5). Estas funciones resultan útiles para entender como varia la densidad en función de la distancia a una partícula de referencia, así como detectar la presencia de estructura cristalina, en la forma de picos discretos.

En la figura 8.12 observamos que $\mathrm{g}(\mathrm{r})$ calculada sobre todas las partículas, exhibe un primer pico relacionado al tamaño de las partículas. Para $V_{S}>0.01$ observamos que $g(r)$ rápidamente oscila alrededor de 1, lo que señala la ausencia de orden. Sin embargo, para $V_{S} \leqslant 0.01$ observamos que existe una periodicidad, cuya altura aumenta (a $r$ constante) a medida que disminuimos $\mathrm{V}_{S}$.

Podemos realizar un estudio discriminando por tipo de partícula, calculando $\mathrm{g}^{\mathrm{F}}(\mathrm{r})$ y $g^{S}(r)$, los cuales presentamos en la figura 8.13. Al discriminar por tipo, observamos que la contribución del orden proviene únicamente de las partículas lentas, mientras que las 


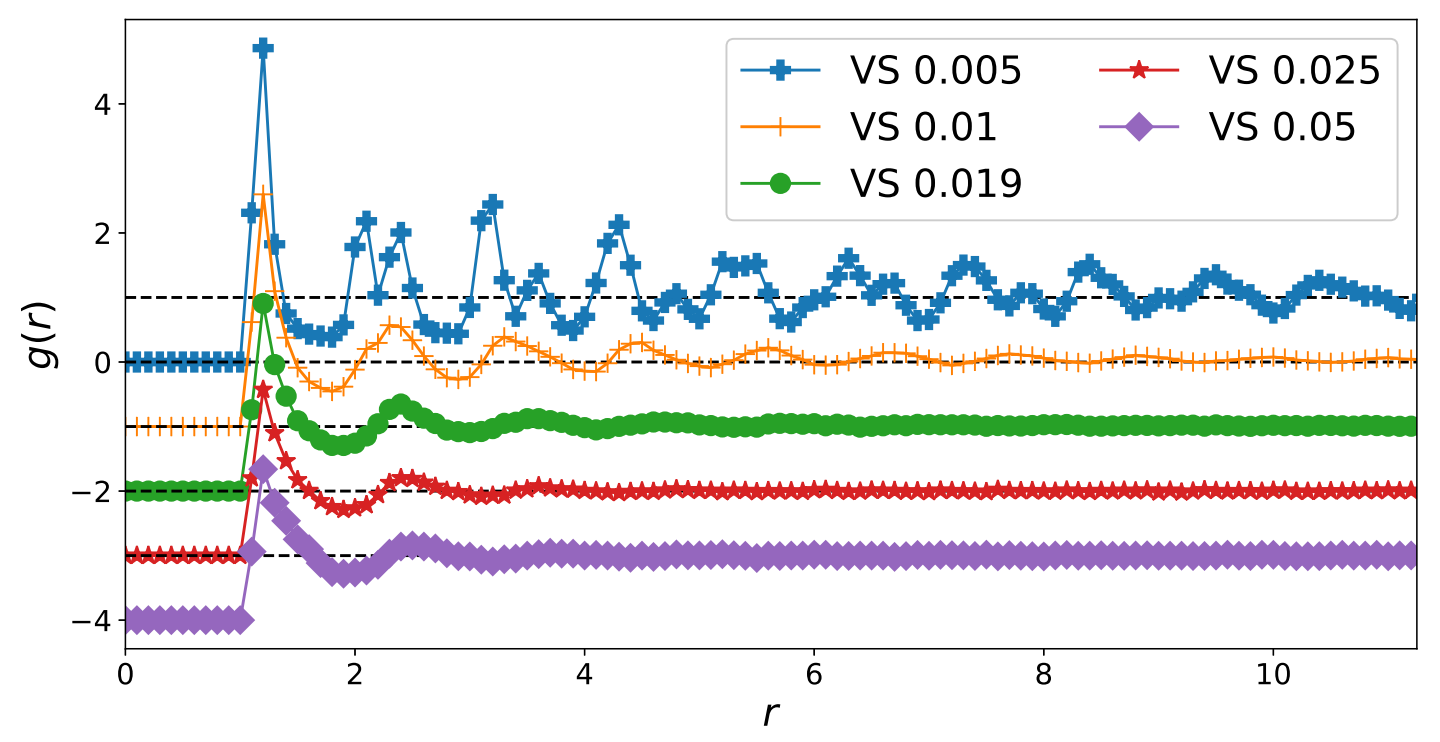

Figura 8.12: Calculo de $\mathrm{g}(\mathrm{r})$ para todas las partículas para valores representativos de $\mathrm{V}_{\mathrm{S}}$. Las curvas tienen un corrimiento para distinguir con claridad los efectos.

partículas rápidas poseen un desorden que resulta independiente de $\mathrm{V}_{\mathrm{S}}$. Los picos para $V_{S}=0.005$, sugieren un ordenamiento cristalino. Lo que implica que al disminuir $V_{S}$, la segregación es seguida de una cristalización de la fase lenta segregada.

Este estudio de correlación espacial mediante g(r), esta complementado con el estudio de la función $S(k)$, presentada en la figura 8.14. Así como $g(r), S(k)$ presenta picos bien definidos para $V_{S}=0.005$, lo que sugiere presencia de estructura cristalina. Nuevamente, si discriminamos las partículas por tipo en $S^{F}(k)$ y $S^{S}(k)$, (ver Figura 8.15) podemos decir que la cristalización es completamente atribuible a las partículas lentas, como ya habíamos observado en las secciones previas. Observando $S^{F}(k)$ no apreciamos diferencias notorias entre las curvas para distintos valores de $V_{S}$. 

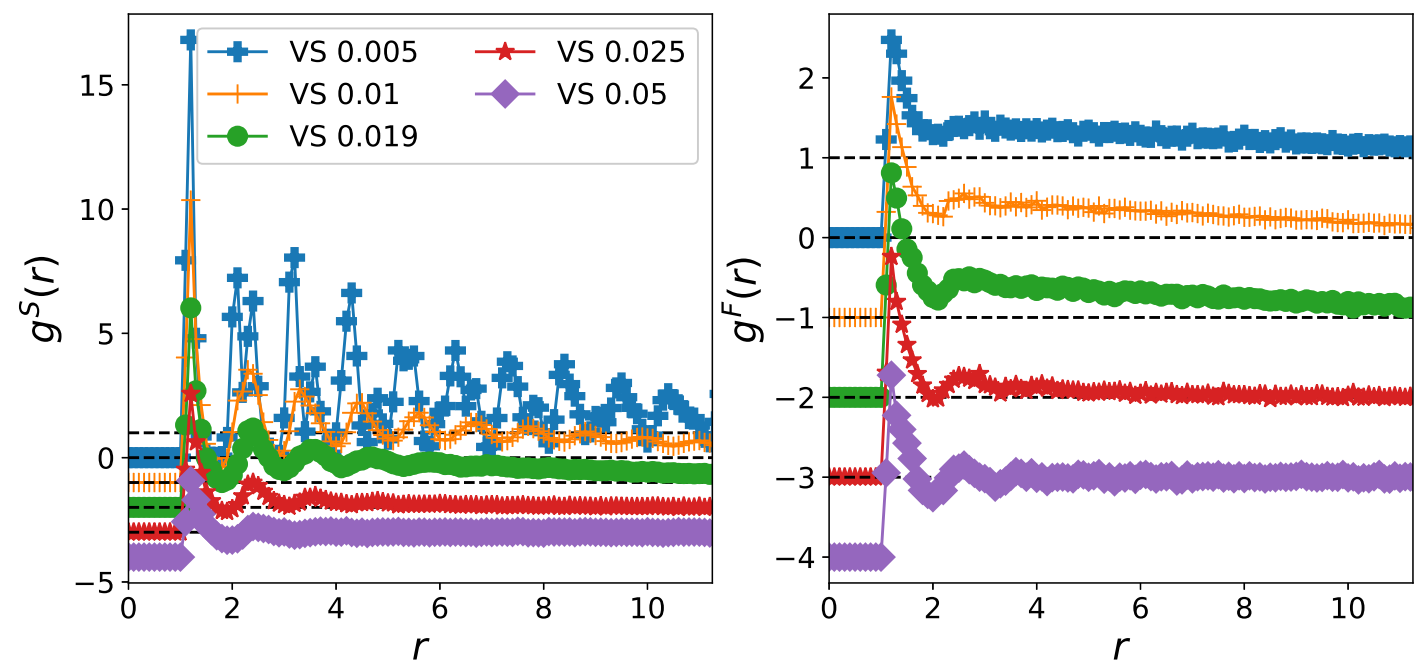

Figura 8.13: Calculo de $g(r)$ discriminando por tipo de partícula. Las curvas tienen un corrimiento para distinguir con claridad los efectos. Observamos ordenamiento en las partículas lentas para $V_{S}$ pequeño, mientras que la estructura de las partículas rápidas no se afecta mucho al variar $\mathrm{V}_{\mathrm{S}}$.

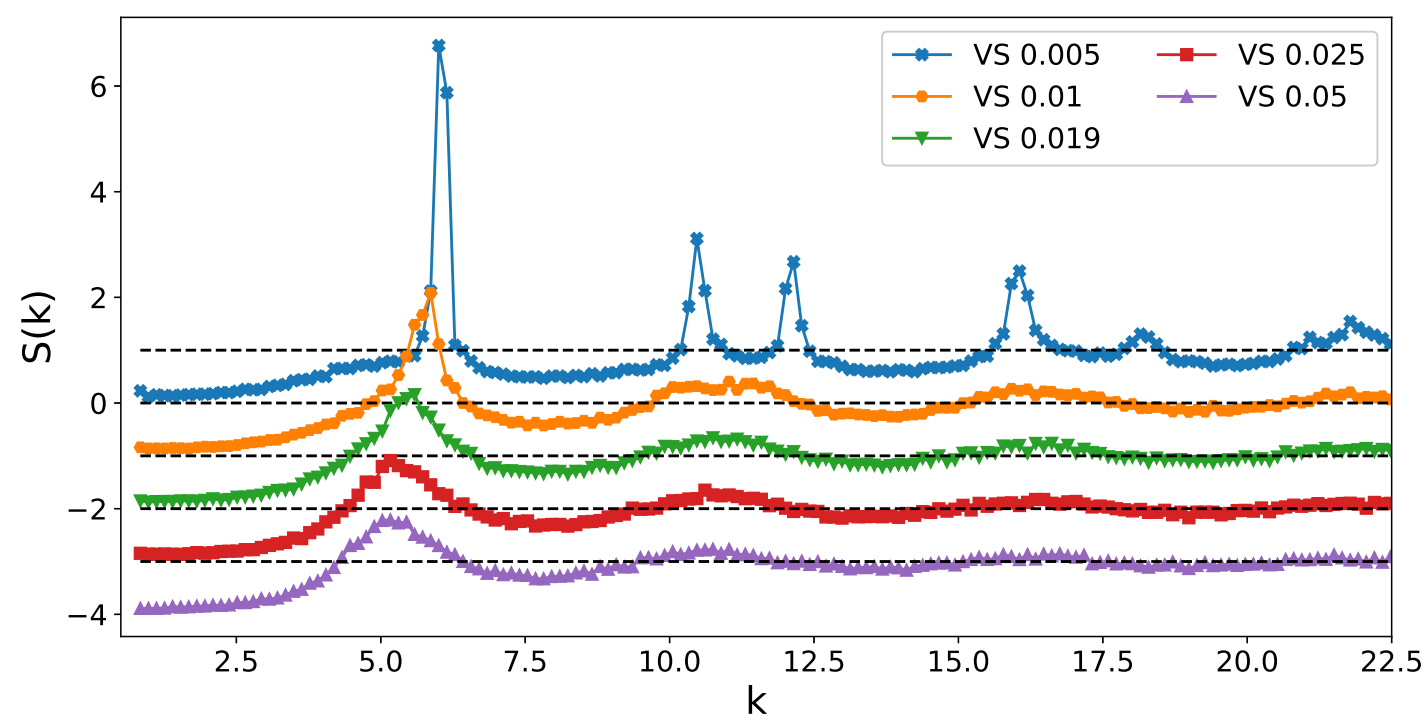

Figura 8.14: Calculo de $S(k)$ para todas las partículas. Las curvas tienen un corrimiento para distinguir con claridad los efectos. Los picos para $V_{S}=0.005$, señalan la existencia de estructura cristalina. 

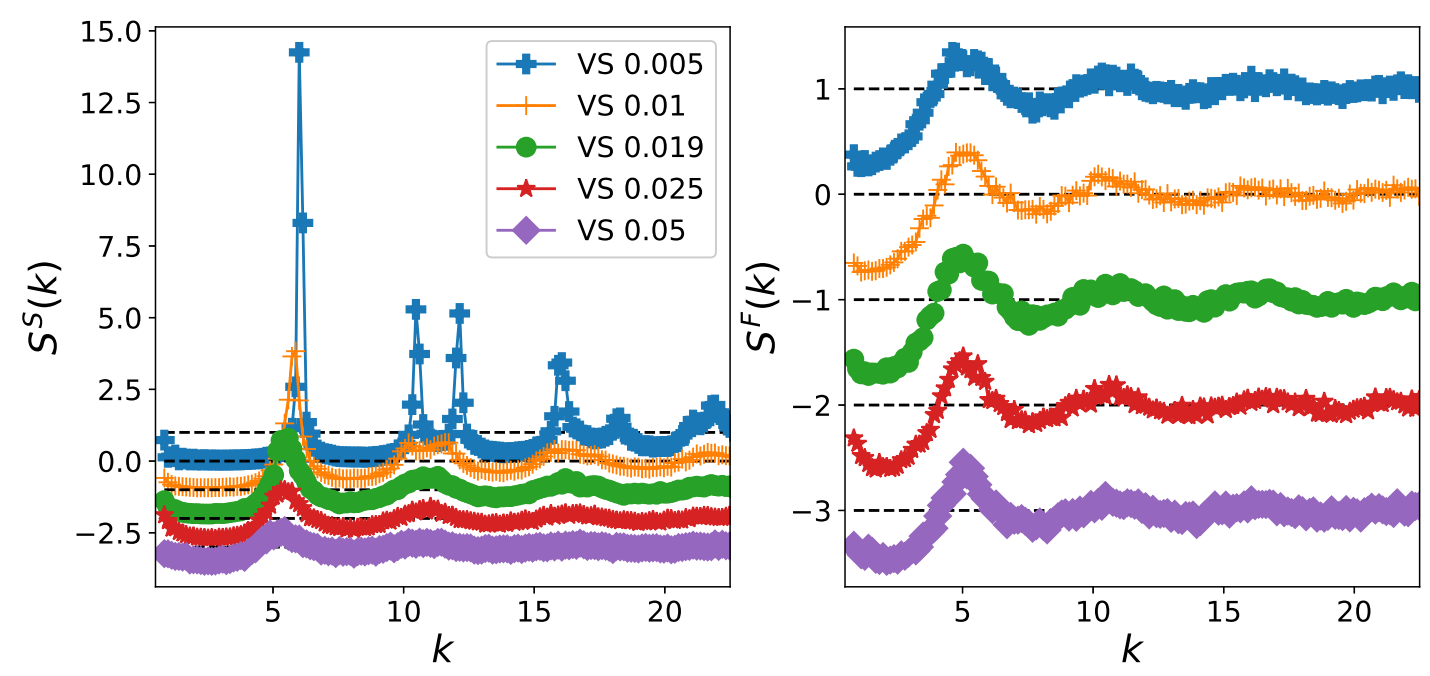

Figura 8.15: Calculo de $S(k)$ para todas las partículas. Las curvas tienen un corrimiento para distinguir con claridad los efectos. Observamos que el ordenamiento cristalino, es debido a las partículas lentas.

Podemos realizar un diagrama (esquemático) de fase para $V_{S}$ y $\rho$ (ver figura 8.16) condensando los resultados presentados. El diagrama describe en forma cualitativa la segregación en función de la densidad y dentro de la región de segregación muestra la presencia de una región con cristalización (solo para las partículas lentas). Fuera de la región de segregación, el sistema se vuelve una mezcla homogénea al aumentar $\mathrm{V}_{\mathrm{S}}$. Para sistemas con $\mathrm{N}=1000$ y $\mathrm{L}>64$ ya no observamos segregación (ver figura 8.5). 


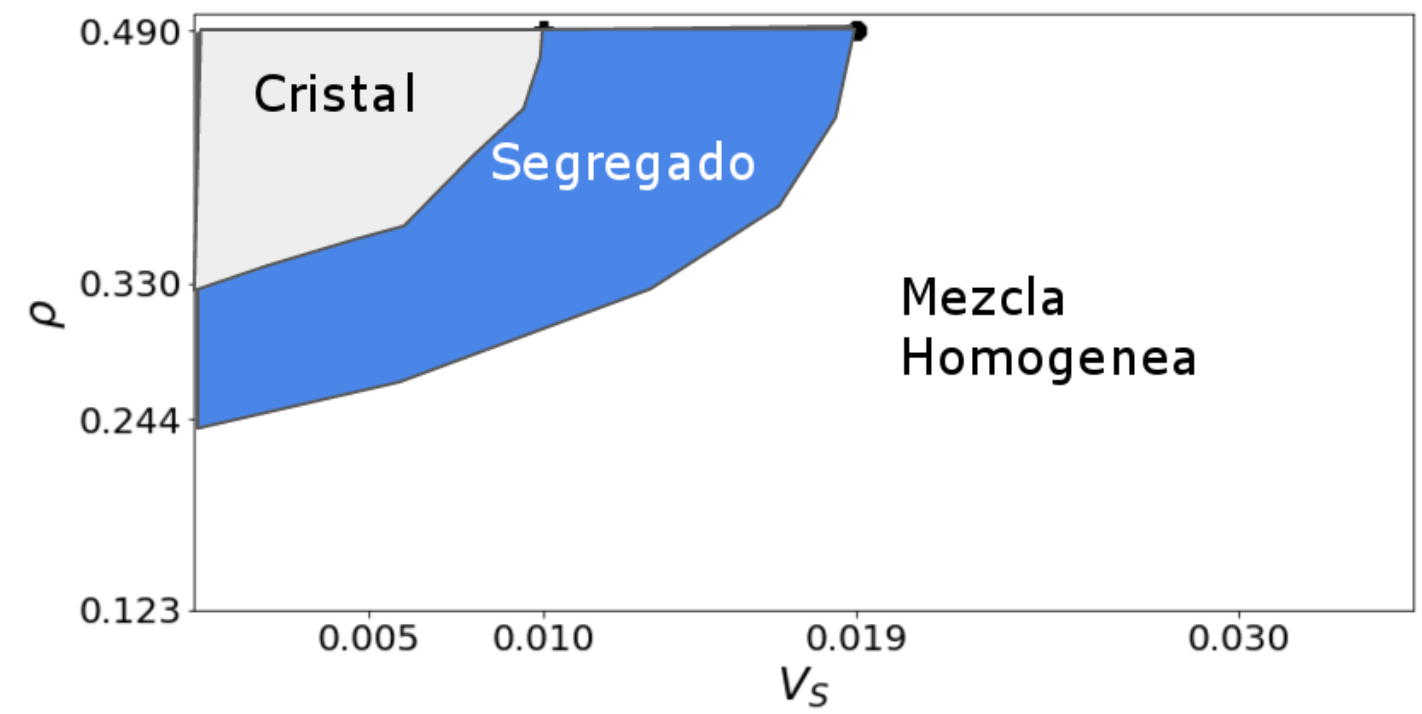

Figura 8.16: Diagrama de fase cualitativo del sistema para variables $V_{S}$ y $\rho$. Cristal representa la zona segregada donde surge una estructura cristalina para las partículas lentas. Fuera de esta región, las partículas se mezclan homogéneamente. Las densidades corresponden a sistemas con $\mathrm{N}=1000$ y tamaños de caja $\mathrm{L}=45,55,64,90$. 


\section{Capítulo 9}

\section{Resultados para Partículas}

\section{Auto-Propulsadas con Volumen Excluido e Interacción Orientacional}

\subsection{Interacción Lennard Jones Junto con Interacción Orien- tacional}

En este capítulo, tomamos el modelo estudiado en la sección anterior y le agregamos una interacción orientacional. Esta interacción, que describiremos en la sección proxima, tiene un radio de corte $\mathrm{R}=\frac{3}{2} \mathrm{r}_{\mathrm{LJ}} \mathrm{y}$ viene dada por las velocidades de las partículas vecinas dentro de ese radio. De esta manera, a medida que dos partículas se aproximan, primero experimentan la interacción orientacional y de seguir aproximándose, la interacción Lennard Jones truncada, como muestra la figura 6.2. Nuevamente utilizamos una caja cuadrada bidimensional de volumen $\mathrm{V}=\mathrm{L}^{2}$, con condiciones de contorno periódicas. Todos los demás parámetros no especificados son los mismos que presentamos en el cuadro 8.1.

\subsubsection{Integración de las Ecuaciones de Movimiento Considerando una Interacción Orientacional}

Utilizaremos una implementación tipo Euler, considerando interacción Lennard Jones truncada $F_{L J}$ e interacción orientacional $F_{O R}$, con el siguiente algoritmo de calculo 
para la j-esima partícula:

$$
\begin{array}{r}
\overrightarrow{\mathrm{F}}_{\mathrm{LJ}}^{j}=\sum_{i} 24 \epsilon \sigma^{6} \mathrm{r}_{i j}^{-8}\left[2 \sigma^{6} \mathrm{r}_{i j}^{-6}-1\right] \overrightarrow{\mathrm{r}}_{i j} \\
\overrightarrow{\mathrm{F}}_{\mathrm{OR}}^{j}=\mathrm{G}^{-1} \sum_{k} \vec{v}_{\mathrm{kj}} \\
\overrightarrow{\mathrm{F}}_{\mathrm{Tot}}^{j}=\overrightarrow{\mathrm{F}}_{\mathrm{LJ}}^{j}+\overrightarrow{\mathrm{F}}_{\mathrm{OR}}^{\mathrm{j}}
\end{array}
$$

Donde $i$ representa las partículas a una distancia $r_{i j}$ de la partícula $j$ con $r_{i j} \leqslant r_{L j}=$ $2^{1 / 6} \sigma$ y $k$ representa a las partículas a una distancia $r_{j k}$ de la partícula $j$ con $r_{j k} \leqslant$ $\frac{3}{2} 2^{1 / 6} \sigma$.

Sea FPR $\in[0,1]$ la fracción de partículas rápidas y $G$ la velocidad media global del sistema dada por $G=F P R v_{0}^{F}+(1-F P R) v_{0}^{S}$. Nuevamente denotamos a las partículas rápidas como $\mathrm{F}$ y a las lentas como $\mathrm{S}$. De manera que la actualización de la posición de la j-esima partícula de tipo T (rápida o lenta) resulta:

$$
\begin{array}{r}
\vec{x}(t+d t)=\vec{x}(t)+\vec{v}(t) d t \\
\overrightarrow{v^{\prime}}=\vec{v}(t)+\vec{F}_{T o t}(t) d t \\
\theta=\operatorname{atan} 2\left(v_{y}^{\prime}, v_{x}^{\prime}\right) \\
\vec{v}(t+d t)=v_{0}^{\top}[\cos \theta \hat{i}+\sin \theta \hat{j}]
\end{array}
$$

Donde $v_{0}^{\top}$ representa la rapidez constante para el tipo de partícula T. Esta integración, al igual que el caso estudiado en la sección 8, posee dos términos de interacción los cuales no están acotados, pero cuyo efecto es el de determinar la dirección del movimiento. El uso de atan2 nos permite extraer la dirección del movimiento sin modificar el módulo de la velocidad de las partículas.

\subsection{Determinación y Clasificación de Estados Estaciona- rios}

Así como en la sección anterior, realizamos una variante de la prueba de condición inicial (ver figura 9.1) para determinar si las realizaciones alcanzan un estado estacionario. Puesto que ahora existen interacciones orientacionales activadas, consideraremos tanto el observable LJSF así como la polarización $\Phi$ (ver Figura 9.2). Recordamos que, 

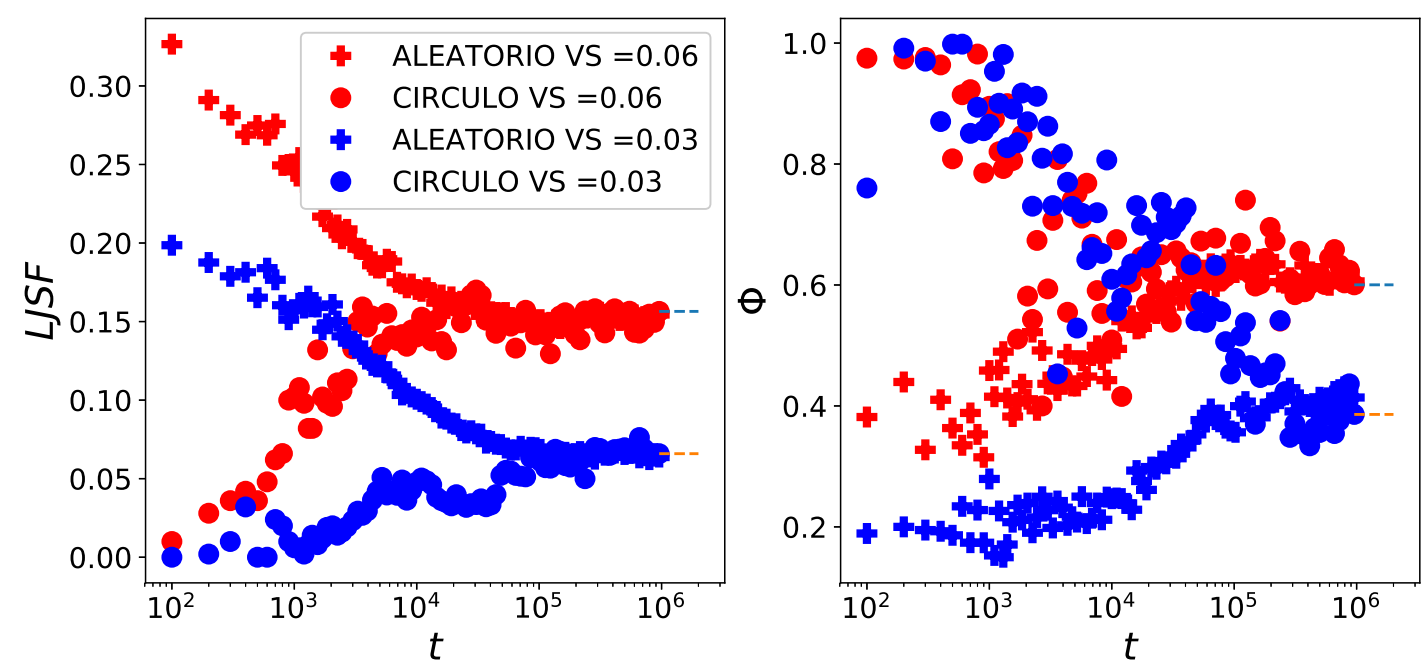

Figura 9.1: Realizamos la prueba de condición inicial usando los observables LJSF y $\Phi$, notamos que para distintos tiempos, el sistema eventualmente siempre se vuelve ergódico.

como se menciono en la sección 6.4 .2 , $\Phi$ viene dado por:

$$
\Phi=N^{-1}\left|\sum_{i=0}^{N} \vec{v}_{i}\right|
$$

Una vez que realizamos las pruebas de condición inicial y verificamos que el sistema estacionó, podemos realizar las medidas estacionarias de LJSF y $_{\infty}=\langle\overline{\Phi(t)}\rangle$.

Podemos estudiar la magnetización $\Phi$ para cada clase de partícula $\left(\Phi_{\infty}^{S}\right.$ considera partículas lentas y $\Phi_{\infty}^{\mathrm{F}}$ considera las partículas rápidas). Como muestra la figura 9.2, para una caja de tamaño $L=45$, observamos que alrededor de $V_{S}=0.015$ hay un mínimo en $\Phi_{\infty}^{\mathrm{F}}$. Para las partículas lentas, el crecimiento continuo de $\Phi_{\infty}^{\mathrm{S}}$, a medida que crece $\mathrm{V}_{\mathrm{S}}$, nos indica que mas y mas dominios de partículas lentas que se orientan en la misma dirección. Combinando esto con la súbita caída de $\operatorname{LJSF}_{\infty}$ para $\mathrm{V}_{\mathrm{S}} \simeq 0.07$ y el súbito incremento en $\Phi_{\infty}^{\mathrm{F}}, \Phi_{\infty}^{\mathrm{S}}$, determinamos que el sistema experimenta una segregación para $\mathrm{V}_{\mathrm{S}} \geqslant 0.07$.

$\mathrm{El}$ análisis de los observables LJSF y $\Phi$, junto con las configuraciones finales de las simulaciones (ver Figura 9.3), nos sugiere una clasificación esquemática del comportamiento estacionario en 3 regímenes:

- $\mathrm{V}_{\mathrm{S}}<0.01$ Las partículas rápidas forman grandes cúmulos orientados (bandada), mientras que las partículas lentas se mantienen mayormente desordenadas orientacionalmente $\left(\Phi^{\mathrm{F}}>\Phi^{\mathrm{S}}\right)$. 

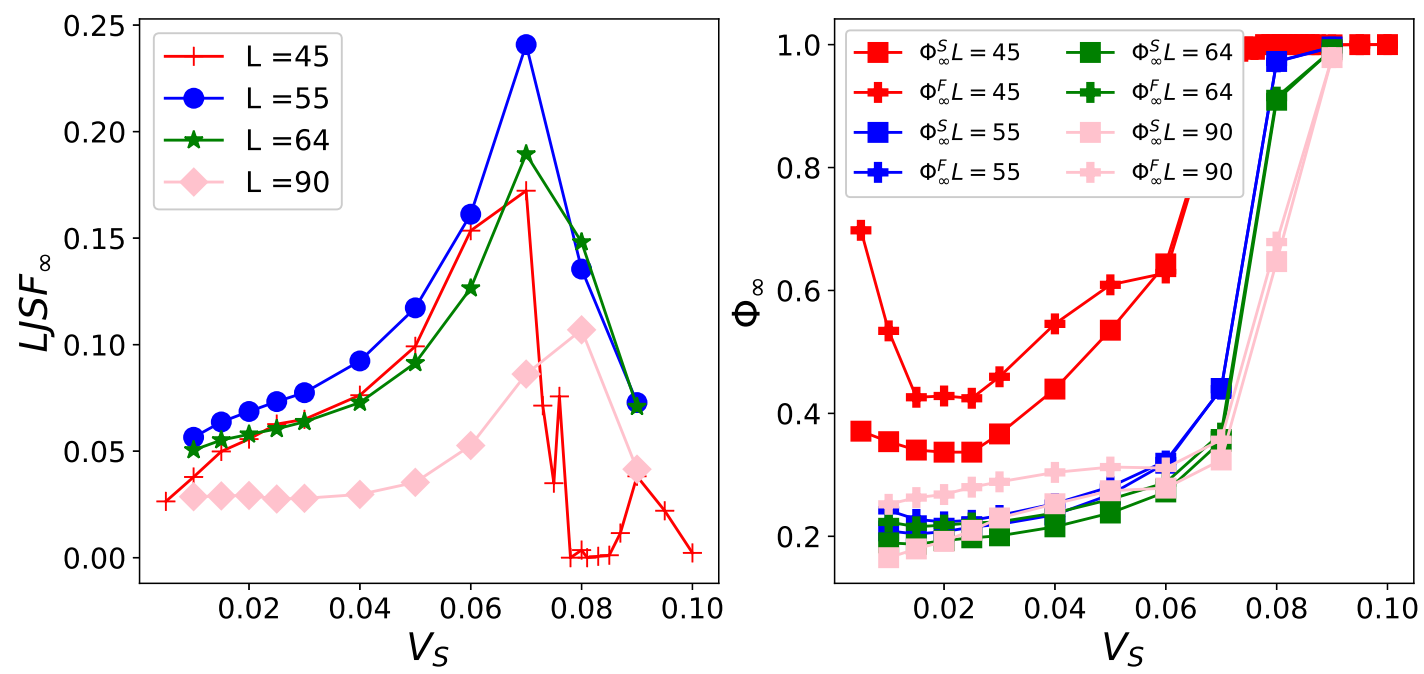

Figura 9.2: Medidas desde condiciones iniciales aleatorias/orden artificial del sistema para distintos valores de $V_{S}$. Si bien los efectos se atenúan ligeramente para $L=90$, observamos que existe un cambio de comportamiento para $V_{S} \gtrsim 0.07$.
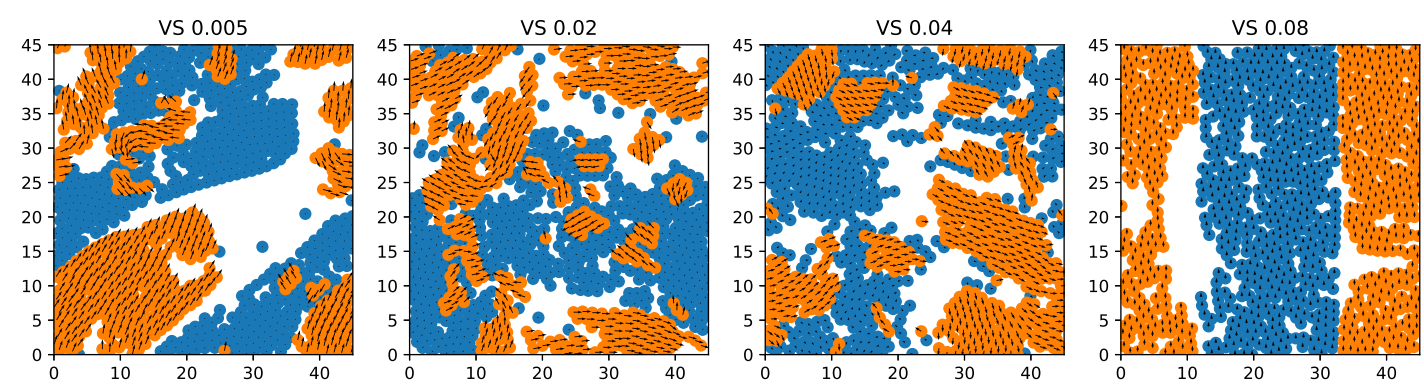

Figura 9.3: Configuraciones finales del sistema con interacciones orientacionales e interacciones Lennard-Jones. Al aumentar $\mathrm{V}_{\mathrm{S}}$ el sistema pasa de una segregación parcial, luego forma cúmulos segregados mas pequeños y finalmente alcanza una segregación total.

- $0.01<\mathrm{V}_{\mathrm{S}}<0.07$ Se forman muchos pequeños cúmulos (aumenta $\mathrm{LJSF}_{\infty}$ ) a medida que las partículas comienzan a orientarse.

- $\mathrm{V}_{\mathrm{S}}>0.07$ Ambos tipos de partículas alcanzan un ordenamiento orientacional total y segregado $\left(\mathrm{LJSF}_{\infty}\right.$ cae$)$.

Resulta interesante notar que, a diferencia del caso sin interacciones orientacionales, para el rango de $V_{S}$ estudiado, el sistema nunca adopta un estado de mezcla homogénea.

Observamos también grandes espacios vacíos, mostrados como regiones en blanco en las representaciones del sistema de la figura 9.3. Creemos que estos espacios se deben al comportamiento colectivo de las partículas rápidas, en las que estas se mueven como 


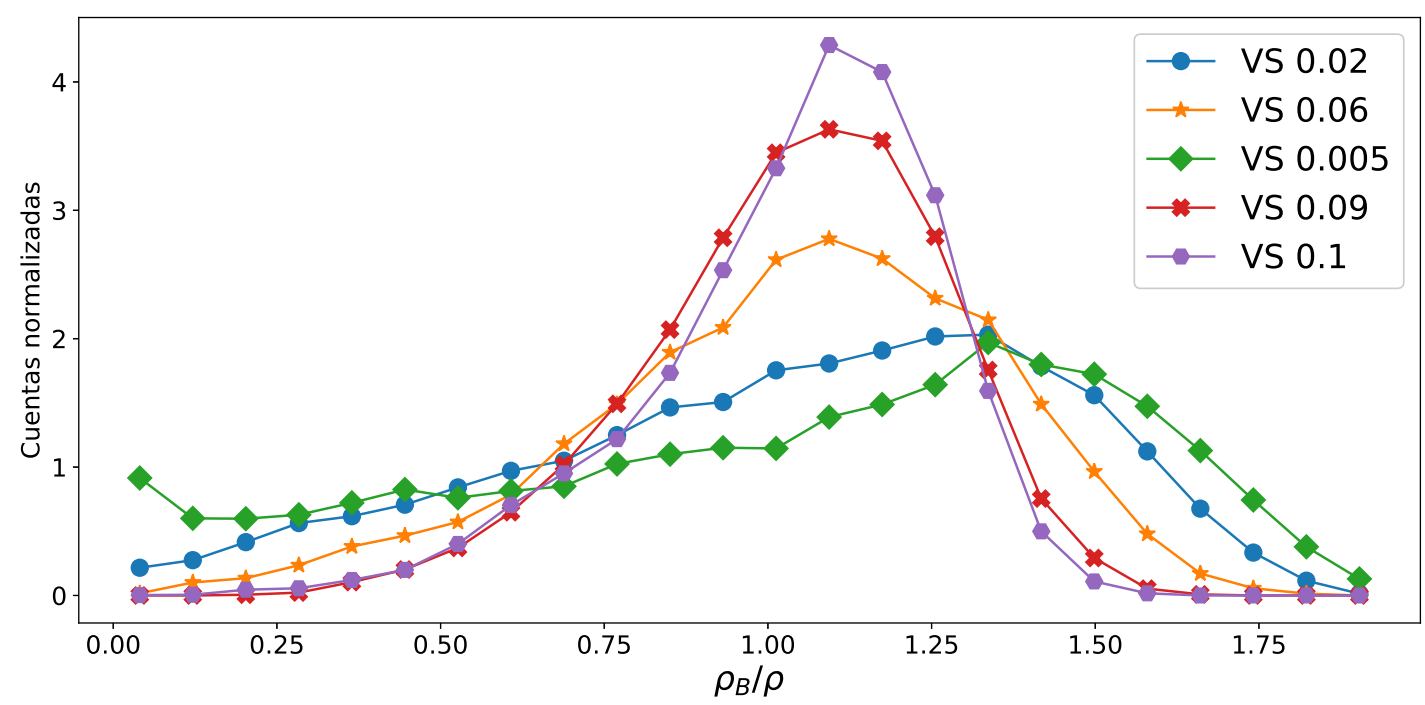

Figura 9.4: Distribuciones locales de densidad. Observamos que a medida que $\mathrm{V}_{\mathrm{S}}$ disminuye, distintas partes del sistema se organizan en densidades locales distintas.

un todo (bandada), liberando espacios que las partículas lentas no son suficientemente veloces para ocupar.

\subsection{Distribuciones Locales de Densidad y Concentración}

Utilizando el análisis de sub-bloques, realizamos una determinación de los comportamientos locales de densidad $\rho_{B}$ y concentración $C_{B}$. Al igual que en la sección anterior, la densidad $\rho$ y concentración global $\mathrm{C}$ se mantienen constantes.

Repitiendo el análisis para los mismos sub-bloques B utilizados en la sección anterior (ver Figura 9.4), observamos que a medida que $V_{S}$ disminuye, el sistema se distribuye en una variedad cada vez mayor de densidades locales. Mientras que cuando hacemos $V_{S} \rightarrow V_{F}=0.1$ las densidades locales $\rho_{B}$ se aproximan por derecha a la densidad global $\rho$. Este corrimiento de la media de $\rho_{\mathrm{B}}$, lo atribuimos a que el algoritmo de sub-bloques excluye del análisis a sub-bloques vacíos (espacios blancos en la figura 9.3). Esto lleva a que en promedio, localmente hay menos espacio para el mismo número de partículas. Lo que explica el ligero incremento de la densidad media $\rho_{\mathrm{B}}$, respecto a la densidad global.

La figura 9.5 confirma la presencia de varios grados de segregación para los valores estudiados. Únicamente el valor $\mathrm{V}_{\mathrm{S}}=\mathrm{V}_{\mathrm{F}}=0.1$ presenta la distribución estilo gaussiana para la concentración local de partículas rápidas $C_{\mathrm{B}}^{\mathrm{F}}$. Esto refuerza el hecho de que, excluyendo $V_{S}=V_{F}$, el sistema no se comporta como una mezcla homogénea para 


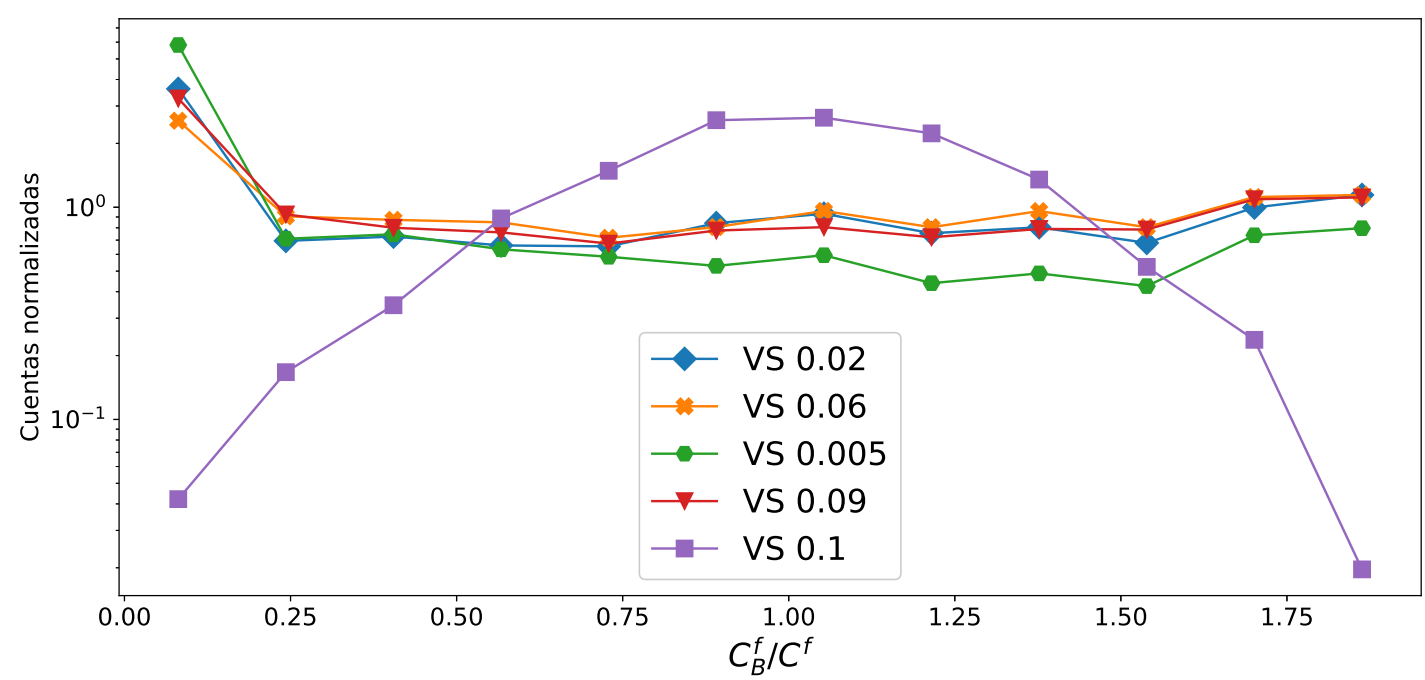

Figura 9.5: Distribuciones locales de concentración de partículas rápidas $\mathrm{C}^{\mathrm{F}}$. Notamos que solo $V_{S}=V_{F}=0.1$ exhibe una distribución de concentraciones tendiendo a la de una mezcla homogénea.

ningún valor de $\mathrm{V}_{\mathrm{S}}$ estudiado.

\subsection{Transición de Segregación y Cumulantes de Binder}

Así como en el estudio anterior, buscaremos determinar una transición de segregación mediante el uso del cumulante de Binder. En particular, observamos que el cumulante para la concentración de partículas rápidas $U\left(C^{F}\right)$ (ver Figura 9.6), presenta una región (no un punto) donde todas las curvas colapsan en $V_{S} \simeq 0.08$. Esta región coincide con los saltos abruptos detectados para $L J S F_{\infty}$ y $\Phi_{\infty}$ para $V_{S} \geqslant 0.07$. Puesto que el cumulante de Binder predice una transición de segundo orden para un punto crítico en el punto donde todas las curvas se cruzan, resulta imposible garantizar que el colapso que observamos se trata de una transición de segundo orden. Sin embargo, podemos observar que la región de $V_{S} \simeq 0.08$ presenta criticalidad. 

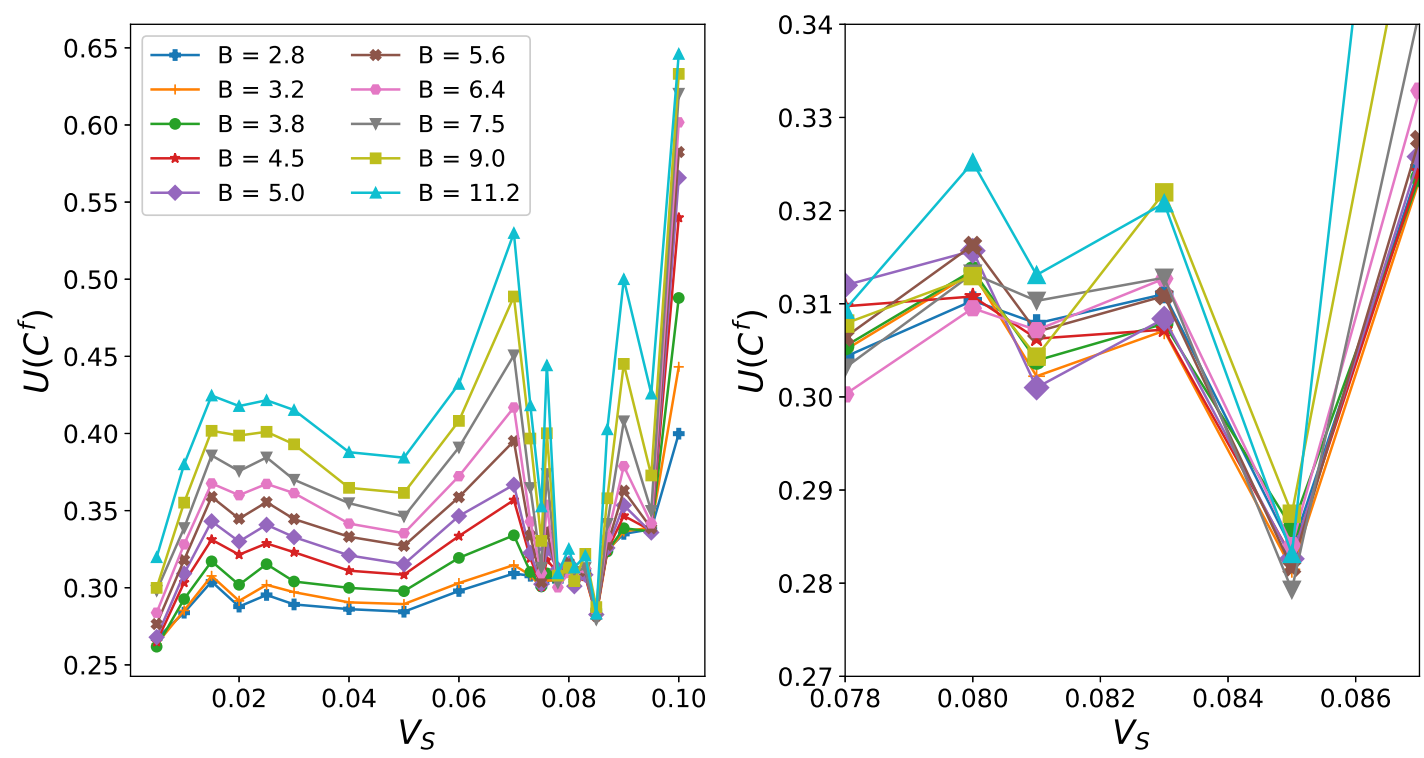

Figura 9.6: Para el cumulante de concentración de partículas rápidas $\mathrm{U}\left(\mathrm{C}^{\mathrm{F}}\right)$, observamos la presencia de una zona de cruce en $V_{S} \simeq 0.08$. Coincide con la zona en que $\Phi$ estacionario se aproxima a 1. RECUADRO: Zoom a la región donde se produce el colapso de sub-bloques de distintos tamaños.

\subsection{Detección de orden mediante $S(k)$ y $g(r)$}

Nuevamente, utilizamos las funciones de correlación espacial estática, $g(r)$ y $S(k)$, para extraer información estructural y así detectar la presencia de orden estructural.

Como muestra la figura 9.7, si bien el estado segregado ordenado se observa para $V_{S}>0.07$, el ordenamiento debido a la interacción orientacional no implica un ordenamiento estructural de cristalización. Un análisis de $\mathrm{g}(\mathrm{r})$ para todas las partículas, no presenta picos definidos y periódicos que decaen al aumentar $\mathrm{V}_{\mathrm{S}}$, como los observados en el caso sin interacción orientacional. En cambio, con interacciones orientacionales, las curvas $\mathrm{g}(\mathrm{r})$ poseen pequeños picos de poca periodicidad, que no presentan gran variación para las distintas $\mathrm{V}_{\mathrm{S}}$ estudiadas.

Sin embargo, un análisis en que discriminamos por tipo de partícula (ver figura 9.8), ilustra una imagen completamente distinta a la presentada para sistemas sin interacciones orientacionales. No solo las partículas lentas, $\mathrm{g}^{\mathrm{S}}(\mathrm{r})$, presentan picos para todo el rango de $V_{S}$, sino que también las partículas rápidas, $g^{F}(r)$, presenta picos que evidencian cierto orden local de corto alcance (poca periodicidad). Atribuimos entonces que las interacciones orientacionales le brindan una estructura de corto alcance a los conjuntos de partículas rápidas y a los conjuntos de partículas lentas.

Para partículas lentas utilizando interacción orientacional, cabe destacar que si bien 


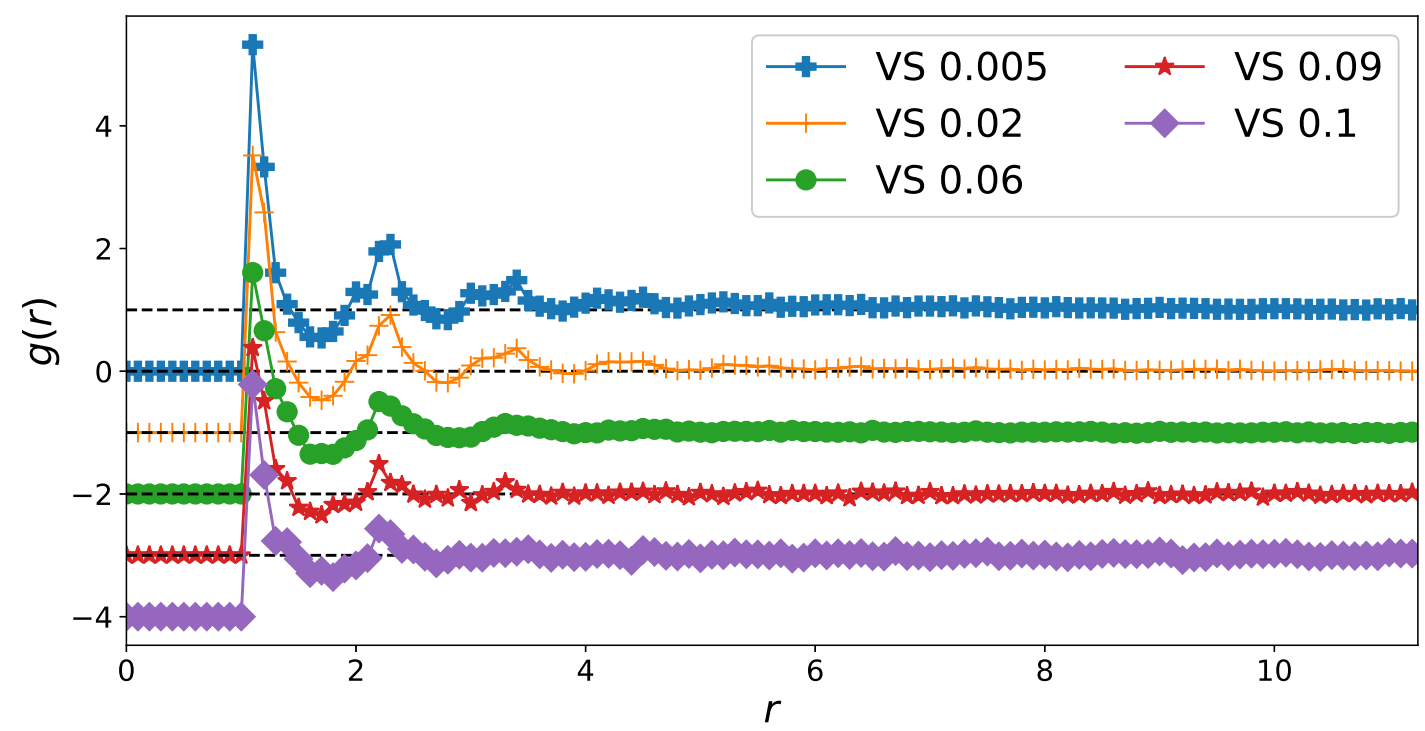

Figura 9.7: g(r) para todas las partículas, con interacción orientacional activada. La presencia de picos que no decaen rápidamente a 1 , muestra que existe cierto ordenamiento de corto alcance aún para $V_{S}>0.01$
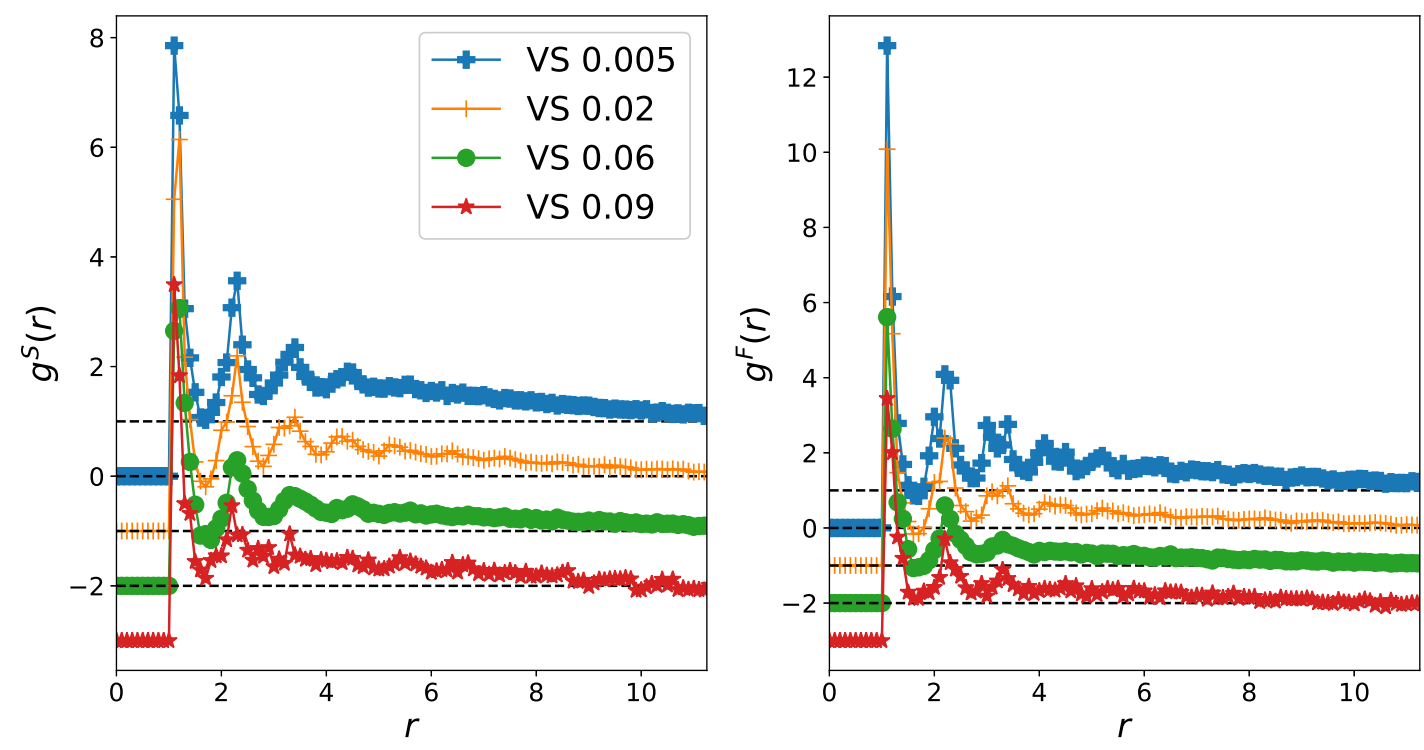

Figura 9.8: $g(r)$ para todas las partículas, con interacción orientacional activada. Notamos que existe un ordenamiento de corto alcance aún para $V_{S}>0.01$ 


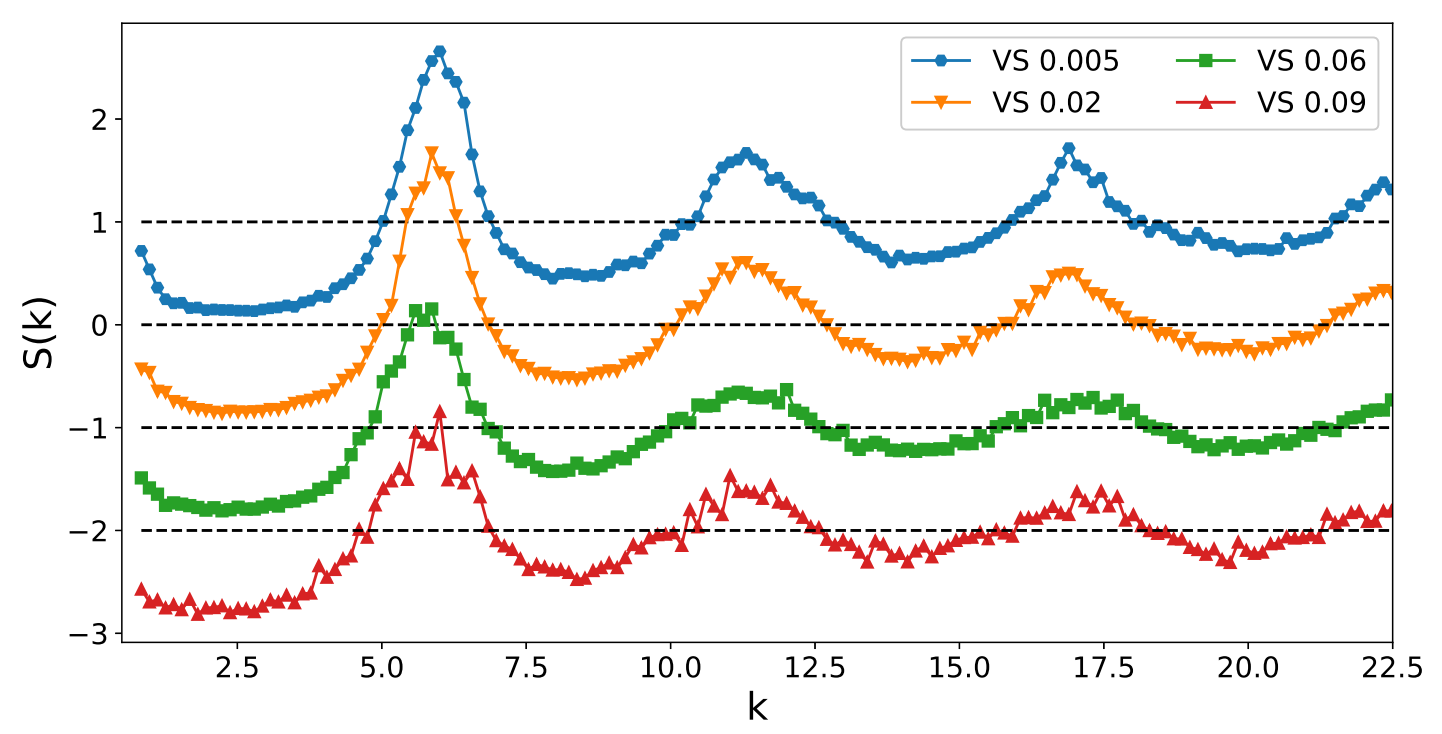

Figura 9.9: $S(k)$ para todas las partículas, con interacción orientacional activada. Notamos que existe un ordenamiento presente para todos los valores de $V_{S}$ estudiados.

hay orden para un amplio rango de $\mathrm{V}_{\mathrm{S}}$, la altura de los picos de dicho ordenamiento se mantiene casi constante, mientras que en el caso sin interacción orientacional, la altura del pico de ordenamiento es el triple de grande para $V_{S}=0.005$ decayendo casi completamente para $\mathrm{V}_{\mathrm{S}}=0.08$.

Podemos extraer las mismas conclusiones estudiando la función de estructura estática $S(k)$ (Figura 9.9). Esta medida, refuerza el hecho de que existe cierta estructura para todo el rango de $\mathrm{V}_{\mathrm{S}}$ estudiado. Sin embargo, comparando con el caso sin interacciones orientacionales (ver Figura 8.14) no observamos picos de gran altura que desaparecen a medida que $V_{S}$ aumenta.

Discriminando por tipo de partícula, podemos calcular $S^{\mathrm{F}}(\mathrm{k})$ y $S^{S}(\mathrm{k}$ ) (ver figura 9.10) y observar que las interacciones orientacionales agregan estructura a ambos tipos de partícula, para todo el rango de valores de $V_{S}$. Esto refuerza la idea de que el orden cristalino de gran alcance está presente en el caso no orientacional a baja $V_{S}$, mientras que el caso orientacional posee un orden de alcance menor pero presente para todo el espectro de $V_{S}$. 

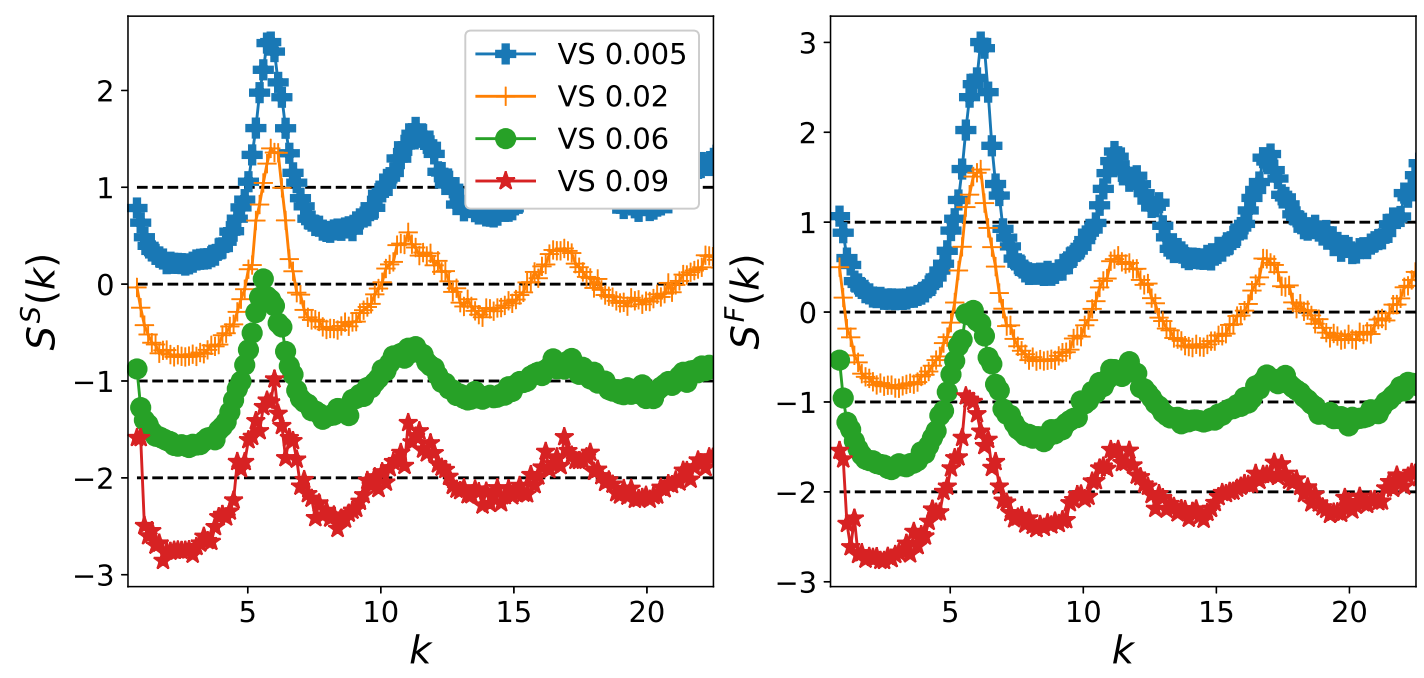

Figura 9.10: $S^{\mathrm{F}}(\mathrm{k})$ para partículas rápidas y $\mathrm{S}^{\mathrm{S}}(\mathrm{k})$ para partículas lentas. Observamos que existen picos para todo el espectro de $V_{S}$ en ambas funciones.

Así como en el caso sin interacción orientacional (ver figura 8.16), podemos realizar un diagrama de fase esquemático para $V_{S}$ y $\rho$ (ver figura 9.11) condensando los resultados presentados en el capítulo. El diagrama describe, en forma cualitativa, la presencia de segregación. En este caso, si disminuimos la densidad $\rho$, la segregación toma lugar cuando las velocidades lentas $V_{S}$ se acercan en valor a la velocidad rápida $V_{F}$. A diferencia del caso no orientacional, el sistema no se vuelve una mezcla completamente homogénea fuera de la zona de segregación, sino que presenta cúmulos ordenados orientacionalmente (ver $\mathrm{LJSF}_{\infty}$ y $\Phi_{\infty}$ en la figura 9.2). Haciendo referencia a la figura 9.3 , podemos pensar que la estructura de bandas $\left(V_{S}=0.08\right)$ se fragmenta al disminuir $\mathrm{v}_{\mathrm{S}}$. 


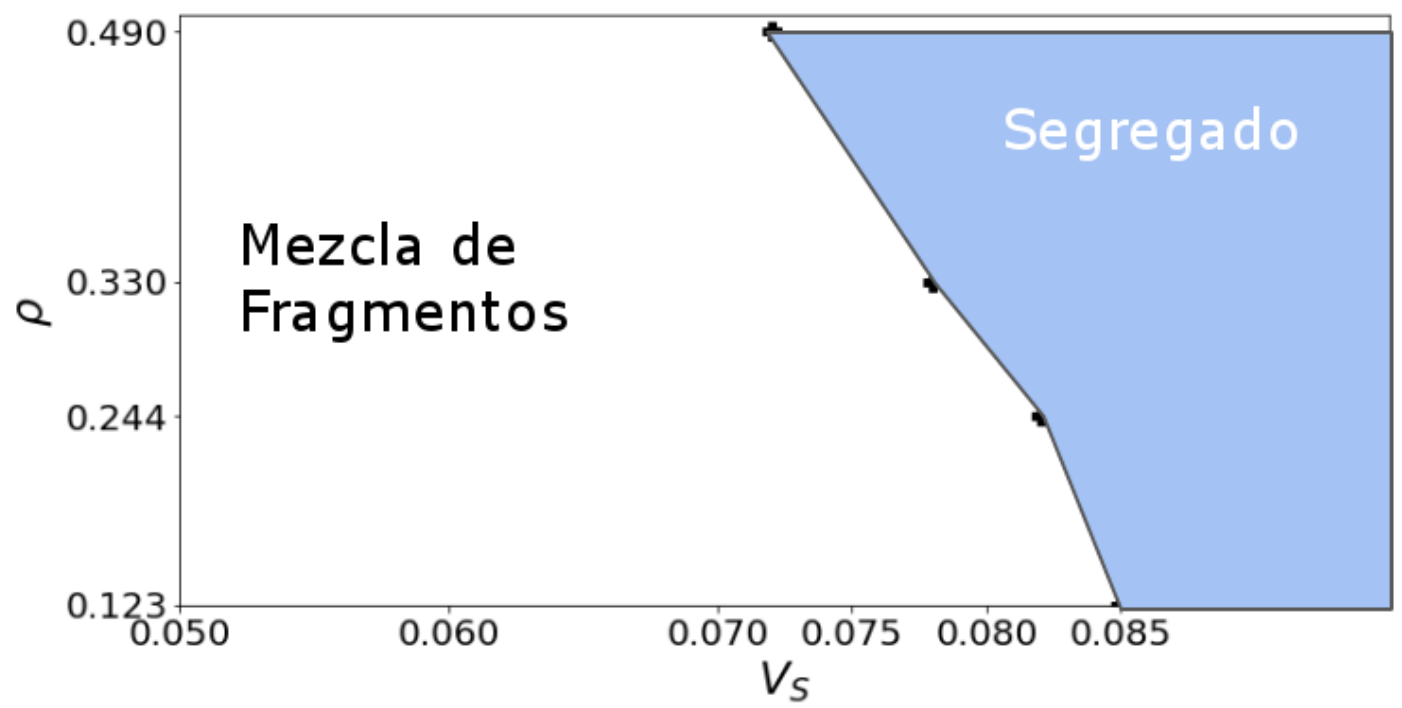

Figura 9.11: Diagrama de fase cualitativo del sistema para variables $V_{\mathrm{S}}$ y $\rho$. Las densidades corresponden a sistemas con $N=1000$ y tamaños de caja $L=45,55,64,90$. Cuando $V_{S} \rightarrow V_{F}$, el sistema presenta segregación. Fuera de la región de segregación, el sistema no es homogéneo. 


\section{Parte III}

\section{Conclusiones Generales}




\section{Capítulo 10}

\section{Conclusiones}

Hemos estudiado, mediante simulaciones computacionales, modelos para líquidos formadores de vidrios (PCTCC y t154) y modelos para partículas auto-propulsadas con volumen excluído (con y sin interacción orientacional). Presentamos las conclusiones de nuestros estudios a continuación.

\subsection{Modelo PCTCC}

En este trabajo de tesis hemos estudiado dos modelos propuestos como modelos de vidrio de retículo. Para el caso de PCTCC si bien observamos que el modelo reproduce muchas de las características de los liquido sobreenfriados, el análisis realizado muestra que el modelo no es apropiado para el estudio del régimen profundamente sobreenfriado.

Hemos mostrado que el límite de metaestabilidad se da a una temperatura no demasiado alejada de la temperatura de fusión, lo que resulta en que dejemos de tener un líquido metaestable para $T \leqslant 0.10 \mu$, el cual esta muy por encima de la estimación que realizamos de $T_{K} \simeq 0.05 \mu$.

Lógicamente, una temperatura de Kauzmann inalcanzable no invalida el estudio termodinámico del sistema (en tanto exista un líquido metaestable), pero para el modelo PCTCC el rango de validez para realizar un estudio es demasiado restringido.

Por otra parte, los estudio del estado de vidrio estructural, donde el modelo está fuera de equilibrio, también presentan severas limitaciones. Mas allá del límite de metaestabilidad, el comportamiento fuera de equilibrio es de crecimiento de dominios ${ }^{1}$, lo cual difiere de lo observado para estudios numéricos y experimentales de vidrios estructurales presentados (ver sección 2.6.4).

\footnotetext{
${ }^{1}$ crecimiento de dominios, del ingles coarsening.
} 
El rango de temperatura entre $T=0.10 \mu$ y la temperatura de fusión corresponde a un comportamiento de vidrio estructural, es decir, un comportamiento donde el sistema fuera de equilibrio lentamente evoluciona hacia un liquido en equilibrio.

Sin embargo, encontramos que las funciones de correlación en el régimen de envejecimiento no cumplen el escaleo $t / t_{w}$, dado que el sistema alcanza el equilibrio muy rápidamente en este rango de temperatura. Cuando es enfriado aún más, la relajación se enlentece y aquí es cuando uno esperaría aproximarse al envejecimiento de un vidrio estructural. Desafortunadamente, es también en este punto cuando el liquido cesa de existir y la dinámica responde necesariamente a engrosamiento (coarsening).

\subsection{Modelo t154}

Las características dinámicas del modelo t154, en particular su dinámica heterogénea, lo hacen un buen candidato para modelo de retículo de un vidrio frágil [Darst et al., 2010]. La relación entre propiedades estructurales y su posible conexión con propiedades termodinámicas y de comportamiento vítreo, ha sido un tema inexplorado que fue el que motivo el detallado estudio de la estructura del t154 a altas densidades.

Hemos computado las fluctuaciones de densidad y dos longitudes de correlación estáticas caracteristicas (la longitud PTS $\xi_{\text {PTS }}$ y una longitud $\xi$ extraida del autosolapamiento Q), las cuales se comportan de manera similar y muestran un crecimiento cuando la densidad incrementa. Este crecimiento, acompañado de incremento en las fluctuaciones del número de partículas y de la compresibilidad para $\rho \gtrsim 0.52$, coincide con el incremento en la heterogeneidad dinámica. Este último, medido por las fluctuaciones del autosolapamiento y por el decaimiento del exponente $\beta$ de la relajación. Es también para $\rho \gtrsim 0.52$ que notamos un incremento en efectos de tamaño en la dinámica de sistemas confinados.

Estas coincidencias apuntan a un origen estructural para el enlentecimiento estilo VFT. Sin embargo, el ordenamiento estructural observado para altas densidades, no se ajusta al escenario termodinámico vítreo como el de la teoría RFOT. En particular, el decaimiento de la correlación PTS es siempre exponencial y el decaimiento del autosolapamiento puede ser explicado por la propuesta de que existe 1 estado, el cual presenta una longitud de correlación comparable con la de PTS. En cambio, el aumento de compresibilidad, aunque modesto en el rango de densidades observadas, apunta a un comportamiento que es mas similar a un enlentecimiento crítico tradicional, quizás debido a una transición de separación de fase, que ocurre mas allá del rango de densidades 
accesible computacionalmente.

En un escenario vítreo gobernado por la termodinámica (RFOT o el esquema de Adam-Gibbs), el tiempo de relajación debería ser exponencial, $\tau \propto \exp \left[\xi^{\psi} / k T\right]$. Esta suposición, no es apoyada por nuestros hallazgos [Hocky et al., 2012]. En cambio, nuestros datos se encuentran mas próximos a una ley de potencia $\tau \sim \xi^{z}$. Los exponentes hallados resultan demasiado grandes para que puedan ser exponentes críticos dinámicos. Sin embargo, estos valores se encuentran muy lejanos a un posible punto critico, como para brindar alguna estimación de exponentes críticos. El punto es que el tiempo de relajación parece crecer con $\xi$ más lentamente que lo esperado de un escenario vítreo activado.

Es por esto que si bien el modelo t154 muestra distintas características de fenomenología vítrea, su comportamiento en altas densidades parece alejarse del de un líquido sobreenfriado, en t154 dicho cambio de comportamiento posiblemente este vinculado a la proximidad de una transición de fase.

\subsection{Partículas Auto-Propulsadas con Volumen Excluído}

Mostramos que el análisis de sub-bloques es una herramienta multipropósito, tanto para reconocer la formación de dominios y estudiar los efectos de densidad local, así como para realizar un estudio de tamaño finito junto con cumulantes de Binder. Mencionamos que el análisis requiere únicamente acceso a las posiciones de las partículas, lo que implica que puede re-hacerse rápidamente con distintos parámetros luego de llevar a cabo una simulación costosa en tiempo para la cual se guardan configuraciones a intervalos frecuentes.

Si bien la interacción de volumen excluído es la misma tanto para partículas rápidas como lentas, es el carácter activo de las partículas el que lleva a una separación de fase cuando la diferencia de velocidades aumenta, tal como se observa en los resultados previos comentados en la sección 6.5.

Notamos también que el efecto de disminuir la densidad mediante el incremento del tamaño de la caja para valores de $\mathrm{L}>45$ disminuye la sensibilidad del sistema al variar $\mathrm{V}_{\mathrm{S}}$. Es decir, si la densidad decrece, la segregación disminuye y eventualmente se vuelve independiente de $V_{S}$.

Las correlaciones estructurales $(\mathrm{g}(\mathrm{r})$ y $\mathrm{S}(\mathrm{k}))$ nos informan de la presencia de una fase cristalina para $V_{S} \leqslant 0.01$. Para $V_{S} \simeq 0.0195$, el cumulante de Binder para la concentración de partículas rápidas $\mathrm{U}\left(\mathrm{C}^{\mathrm{F}}\right)$, presenta un cruce de las curvas para distintos tamaños de bloque, lo que sugiere una transición de fase de segundo orden (segregación). 
Mediante un análisis en el mismo rango de $\mathrm{V}_{\mathrm{S}}$ para un cambio en la concentración global, para FPR $=0.25,0.35,0.5$, observamos que la intersección en el cumulante de concentración de las partículas rápidas se deslocaliza. Esto sugiere que solo podemos garantizar que existe una transición de segundo orden para $\mathrm{FPR}=0.5$.

Así como con $\mathrm{U}\left(\mathrm{C}^{\mathrm{F}}\right)$, mediante el estudio de densidades locales, observamos la separación del sistema homogéneo en una fase diluida y una fase densa para $V_{S} \leqslant 0.0195$. Observamos que la diferencia de densidades se comporta como una ley de potencias en función de $V_{S}$.

\subsection{Partículas Auto-Propulsadas con Volumen Excluído e Interacción Orientacional}

En este caso, el análisis de sub-bloques nos permite detectar que el sistema con interacciones orientacionales posee un comportamiento fundamentalmente distinto al caso donde solo existen efectos de volumen excluído.

El análisis de concentración local exhibe un sistema que se encuentra segregado con dominios cuyo tamaño varia con $V_{S}$. Esta información puede recuperarse a través del estudio de la magnetización discriminando por tipo de partícula. Además, observamos que los efectos de segregación no se aplacan al disminuir la densidad del sistema (aumentando L).

Resulta notorio que el comportamiento de bandada en las partículas rápidas, tiene el efecto de generar una estructura de corto alcance, que es detectada por las funciones de correlación estáticas $g(r)$ y $S(k)$.

Observando el cumulante de concentración para las partículas rápidas $\mathrm{U}\left(\mathrm{C}^{\mathrm{F}}\right)$, notamos que se produce una región de criticalidad en la vecindad de $\mathrm{V}_{\mathrm{S}} \simeq 0.08$. Mediante el estudio asintótico de la magnetización, podemos verificar que es para esa región de $V_{S}$ que ambas clases de partículas se ordenan orientacionalmente y el sistema se segrega completamente en franjas.

\subsection{Conclusiones Generales}

En resumen, se realizó un estudio numérico de modelos de vidrios de retículo, como objeto de estudio para investigar el comportamiento de los líquidos formadores de vidrios. Observamos que si bien ambos modelos presentan fenomenología vítrea, resultan inadecuados para un estudio de sobreenfriamiento profundo. Puesto que es en dicha región donde se presentan los fenómenos de interés de las teorías propuestas creemos 
que estos modelos de retículo, si bien poseen reglas termodinámicas y son fáciles de simular, resultan limitados para el estudio de la transición vítrea.

Por otra parte, el estudio de una mezcla binaria de partículas autopropulsadas con volumen excluído, produce el efecto de segregación discutido en la literatura, observando segregación inducida no por diferencia de tamaño de partículas, como se suele observar en sistemas pasivos, sino únicamente por su diferencia de velocidad. El sistema puede ser claramente discriminado en una fase homogénea $\left(\right.$ alta $V_{S}$ ) y una fase heterogénea (baja $V_{S}$ ), la cual incluso da evidencia de cristalización creciente para el caso de las partículas lentas.

Verificamos que el efecto de segregación por velocidad se presenta de otra manera en el caso que las interacciones orientacionales están activadas. En este caso, $V_{S}$ controla la heterogeneidad, es decir tamaño de dominio de las partículas. A diferencia del caso no orientacional, este sistema no presenta un ordenamiento cristalino de alcance creciente para ninguna clase de partículas o $\mathrm{V}_{\mathrm{S}}$.

A lo largo de esta tesis hemos estudiado dos problemas relacionados al no equilibrio, desde un enfoque de materia condensada. El estudio de correlaciones temporales y el uso de condiciones de contorno amorfas, para los líquidos sobreenfriados, así como las correlaciones espaciales y el análisis de sub-bloques en el régimen estacionario para las partículas autopropulsadas prueban ser herramientas centrales para este análisis.

Para el caso de los líquidos formadores de vidrio, concluimos que el uso de estos enfoques conforma una determinación de rango de validez de modelos rápidos y simples de simular en el retículo. El uso de reglas termodinámicas, en vez de cinéticas, resulta ser un camino válido para estudiar el régimen sobreenfriado pero, para los modelos estudiados en este trabajo, resulta insuficiente para un comportamiento de sobreenfriamiento profundo que pueda brindarnos información de una transición vítrea.

Durante el desarrollo de la presente Tesis Doctoral fueron publicados los resultados expuestos del modelo PCTCC en el capitulo 4 en la referencia [Seif et al., 2015], los resultados expuestos del modelo t154 en el capitulo 5, se encuentran en prensa y una versión desactualizada puede encontrarse en la referencia [Seif and Grigera, 2016]. Se encuentran en preparación otros trabajos relativos a los estudios de materia activa detallados en los capítulos 8 y 9. 


\section{Bibliografía}

[Par, 1997] (1997). Animal Groups in Three Dimensions: How Species Aggregate (Psychiatry and Medicine). Cambridge University Press.

[Enc, 2004] (2004). Encyclopedia of Nonlinear Science. Routledge.

[Albano et al., 2011] Albano, E. V., Bab, M. A., Baglietto, G., Borzi, R. A., Grigera, T. S., Loscar, E. S., Rodriguez, D. E., Puzzo, M. L. R., and Saracco, G. P. (2011). Study of phase transitions from short-time non-equilibrium behaviour. Rep. Prog. Phys., 74(2):026501.

[Anderson, 1989] Anderson, P. W. (1989). Spin glass v: Real power brought to bear. Physics Today, 42(7):9-11.

[Baglietto and Albano, 2009] Baglietto, G. and Albano, E. V. (2009). Nature of the order-disorder transition in the vicsek model for the collective motion of self-propelled particles. Phys. Rev. E, 80:050103.

[Barrat, 2003] Barrat, J.L., H. J. (2003). Basic Concepts for Simple and Complex Liquids. Cambridge University Press.

[Barrat and Latz, 1990] Barrat, J. L. and Latz, A. (1990). Mode coupling theory for the glass transition in a simple binary mixture. Journal of Physics: Condensed Matter, 2(18):4289-4295.

[Berthier and Biroli, 2011] Berthier, L. and Biroli, G. (2011). Theoretical perspective on the glass transition and amorphous materials. Rev. Mod. Phys., 83:587-645.

[Berthier et al., 2011] Berthier, L., Biroli, G., Bouchaud, J.-P., Cipelletti, L., and van Saarloos, W., editors (2011). Dynamical Heterogeneities in Glasses, Colloids, and Granular Media. Oxford University Press. 
[Berthier et al., 2012] Berthier, L., Biroli, G., Coslovich, D., Kob, W., and Toninelli, C. (2012). Finite-size effects in the dynamics of glass-forming liquids. Phys. Rev. E, 86:031502.

[Berthier and Witten, 2009] Berthier, L. and Witten, T. A. (2009). Compressing nearly hard sphere fluids increases glass fragility. EPL (Europhysics Letters), 86(1):10001.

[Bialké et al., 2013] Bialké, J., Löwen, H., and Speck, T. (2013). Microscopic theory for the phase separation of self-propelled repulsive disks. EPL (Europhysics Letters), 103(3):30008.

[Binder, 1981] Binder, K. (1981). Finite size scaling analysis of ising model block distribution functions. Zeitschrift für Physik B Condensed Matter, 43:119-140.

[Binder et al., 1999] Binder, K., Baschnagel, J., Bennemann, C., and Paul, W. (1999). Monte carlo and molecular dynamics simulation of the glass transition of polymers. Journal of Physics: Condensed Matter, 11(10A):A47.

[Biroli et al., 2008] Biroli, G., Bouchaud, J.-P., Cavagna, A., Grigera, T. S., and Verrocchio, P. (2008). Thermodynamic signature of growing amorphous order in glassforming liquids. Nature Physics, 4(10):771-775.

[Biroli and Mézard, 2001] Biroli, G. and Mézard, M. (2001). Lattice glass models. Physical Review Letters, 88(2).

[Calabrese and Gambassi, 2005] Calabrese, P. and Gambassi, A. (2005). Ageing properties of critical systems. Journal of Physics A: Mathematical and General, 38(18):R133-R193.

[Castellani and Cavagna, 2005] Castellani, T. and Cavagna, A. (2005). Spin-Glass Theory for Pedestrians.

[Cates and Tailleur, 2015] Cates, M. E. and Tailleur, J. (2015). Motility-induced phase separation. Annual Review of Condensed Matter Physics, 6(1):219-244.

[Cavagna, 2009] Cavagna, A. (2009). Supercooled liquids for pedestrians. Physics Reports, 476(4-6):51-124.

[Cavagna et al., 2007] Cavagna, A., Grigera, T. S., and Verrocchio, P. (2007). Mosaic Multistate Scenario Versus One-State Description of Supercooled Liquids. Phys. Rev. Lett., 98(18):187801. 
[Cavagna et al., 2010] Cavagna, a., Grigera, T. S., and Verrocchio, P. (2010). Numerical simulations of liquids with amorphous boundary conditions. Journal of Statistical Mechanics: Theory and Experiment, 2010(10):P10001.

[Cavagna et al., 2012] Cavagna, A., Grigera, T. S., and Verrocchio, P. (2012). Dynamic relaxation of a liquid cavity under amorphous boundary conditions. The Journal of Chemical Physics, 136(20):204502.

[Couzin et al., 2005] Couzin, I. D., Krause, J., Franks, N. R., and Levin, S. A. (2005). Effective leadership and decision-making in animal groups on the move. Nature, 433(7025):513-516.

[Cross and Hohenberg, 1993] Cross, M. C. and Hohenberg, P. C. (1993). Pattern formation outside of equilibrium. Rev. Mod. Phys., 65:851-1112.

[Czirók et al., 1996] Czirók, A., Ben-Jacob, E., Cohen, I., and Vicsek, T. (1996). Formation of complex bacterial colonies via self-generated vortices. Phys. Rev. E, 54:1791-1801.

[Darst et al., 2010] Darst, R. K., Reichman, D. R., and Biroli, G. (2010). Dynamical heterogeneity in lattice glass models. The Journal of Chemical Physics, 132(4):044510.

[Das et al., 2003] Das, S. K., Horbach, J., and Binder, K. (2003). Transport phenomena and microscopic structure in partially miscible binary fluids: A simulation study of the symmetrical lennard-jones mixture. The Journal of Chemical Physics, 119(3):1547-1558.

[Das, 2004] Das, S. P. (2004). Mode-coupling theory and the glass transition in supercooled liquids. Reviews of Modern Physics, 76(3):785-851.

[Debenedetti and Stillinger, 2001] Debenedetti, P. G. and Stillinger, F. H. (2001). Supercooled liquids and the glass transition. Nature, 410(6825):259-267.

[Edgeworth et al., 1984] Edgeworth, R., Dalton, B. J., and Parnell, T. (1984). The pitch drop experiment. European Journal of Physics, 5(4):198-200.

[Ediger et al., 1996] Ediger, M. D., Angell, C. A., and Nagel, S. R. (1996). Supercooled liquids and glasses. The Journal of Physical Chemistry, 100(31):13200-13212.

[Ediger and Harrowell, 2012] Ediger, M. D. and Harrowell, P. (2012). Perspective: Supercooled liquids and glasses. The Journal of Chemical Physics, 137(8):080901. 
[Fily and Marchetti, 2012] Fily, Y. and Marchetti, M. C. (2012). Athermal phase separation of self-propelled particles with no alignment. Physical Review Letters, 108(23).

[Fischer et al., 2005] Fischer, H. E., Barnes, A. C., and Salmon, P. S. (2005). Neutron and x-ray diffraction studies of liquids and glasses. Reports on Progress in Physics, 69(1):233-299.

[Garrahan and Chandler, 2002] Garrahan, J. P. and Chandler, D. (2002). Geometrical explanation and scaling of dynamical heterogeneities in glass forming systems. Physical Review Letters, 89(3).

[Goldenfeld, 1992] Goldenfeld, N. (1992). Lectures On Phase Transitions And The Renormalization Group (Frontiers in Physics). Addison-Wesley.

[Goldstein, 1980] Goldstein, H. (1980). Classical Mechanics (Addison-Wesley series in physics). Addison-Wesley.

[Gotze and Sjogren, 1992] Gotze, W. and Sjogren, L. (1992). Relaxation processes in supercooled liquids. Reports on Progress in Physics, 55(3):241-376.

[Gradenigo et al., 2013] Gradenigo, G., Trozzo, R., Cavagna, A., Grigera, T. S., and Verrocchio, P. (2013). Static correlations functions and domain walls in glassforming liquids: The case of a sandwich geometry. The Journal of Chemical Physics, 138(12):12A509.

[Grégoire and Chaté, 2004] Grégoire, G. and Chaté, H. (2004). Onset of collective and cohesive motion. Phys. Rev. Lett., 92:025702.

[Gruler et al., 1999] Gruler, H., Dewald, U., and Eberhardt, M. (1999). Nematic liquid crystals formed by living amoeboid cells. The European Physical Journal B, 11(1):187.

[Hansen and McDonald, 1990] Hansen, J. P. and McDonald, I. (1990). Theory of Simple Liquids. Academic, London.

[Heitjans and Kärger, 2006] Heitjans, P. and Kärger, J. (2006). Diffusion in Condensed Matter: Methods, Materials, Models. SpringerLink: Springer e-Books. Springer Berlin Heidelberg.

[Henkel and Pleimling, 2011] Henkel, M. and Pleimling, M. (2011). Non-Equilibrium Phase Transitions: Volume 2: Ageing and Dynamical Scaling Far from Equilibrium. Theoretical and Mathematical Physics. Springer Netherlands. 
[Hocky et al., 2012] Hocky, G. M., Markland, T. E., and Reichman, D. R. (2012). Growing point-to-set length scale correlates with growing relaxation times in model supercooled liquids. Physical Review Letters, 108(22).

[Huang et al., 2006] Huang, Z.-Y., Merlitz, H., and Wu, C.-X. (2006). Phase separation of a binary two-dimensional core-softened fluid. The Journal of Chemical Physics, 124(23):234506.

[Julicher et al., 2007] Julicher, F., Kruse, K., Prost, J., and Joanny, J. (2007). Active behavior of the cytoskeleton. Physics Reports, 449(1-3):3-28.

[Jungblut and Dellago, 2011] Jungblut, S. and Dellago, C. (2011). Crystallization of a binary lennard-jones mixture. The Journal of Chemical Physics, 134(10):104501.

[Kirkpatrick et al., 1989] Kirkpatrick, T. R., Thirumalai, D., and Wolynes, P. G. (1989). Scaling concepts for the dynamics of viscous liquids near an ideal glassy state. Physical Review A, 40(2):1045-1054.

[Kob and Andersen, 1993] Kob, W. and Andersen, H. C. (1993). Kinetic lattice-gas model of cage effects in high-density liquids and a test of mode-coupling theory of the ideal-glass transition. Physical Review E, 48(6):4364-4377.

[Kurchan and Levine, 2009] Kurchan, J. and Levine, D. (2009). Correlation length for amorphous systems.

[Landau and Binder, 2014] Landau, D. P. and Binder, K. (2014). A Guide to Monte Carlo Simulations in Statistical Physics. Cambridge University Press.

[Levis and Berthier, 2014] Levis, D. and Berthier, L. (2014). Clustering and heterogeneous dynamics in a kinetic monte carlo model of self-propelled hard disks. Phys. Rev. E, 89:062301.

[Lindsey and Patterson, 1980] Lindsey, C. P. and Patterson, G. D. (1980). Detailed comparison of the williams-watts and cole-davidson functions. The Journal of Chemical Physics, 73(7):3348-3357.

[Loscar and Albano, 2003] Loscar, E. and Albano, E. V. (2003). Critical behaviour of irreversible reaction systems. Reports on Progress in Physics, 66(8):1343-1382.

[Loscar et al., 2009] Loscar, E. S., Ferrero, E. E., Grigera, T. S., and Cannas, S. A. (2009). Nonequilibrium characterization of spinodal points using short time dynamics. The Journal of Chemical Physics, 131(2):024120. 
[Loscar et al., 2017] Loscar, E. S., Martin, D. A., and Grigera, T. S. (2017). Stability limits for the supercooled liquid and superheated crystal of lennard-jones particles. The Journal of Chemical Physics, 147(3):034504.

[Lu and Szpunar, 1997] Lu, J. and Szpunar, J. A. (1997). Applications of the embedded-atom method to glass formation and crystallization of liquid and glass transition-metal nickel. Philosophical Magazine A, 75(4):1057-1066.

[Lubchenko and Wolynes, 2007] Lubchenko, V. and Wolynes, P. G. (2007). Theory of structural glasses and supercooled liquids. Annual Review of Physical Chemistry, $58(1): 235-266$.

[Marchetti et al., 2013] Marchetti, M. C., Joanny, J. F., Ramaswamy, S., Liverpool, T. B., Prost, J., Rao, M., and Simha, R. A. (2013). Hydrodynamics of soft active matter. Reviews of Modern Physics, 85(3):1143-1189.

[Marro and Dickman, 2005] Marro, J. and Dickman, R. (2005). Nonequilibrium Phase Transitions in Lattice Models (Collection Alea-Saclay: Monographs and Texts in Statistical Physics). Cambridge University Press.

[Masiewicz et al., 2015] Masiewicz, E., Grzybowski, A., Grzybowska, K., Pawlus, S., Pionteck, J., and Paluch, M. (2015). Adam-gibbs model in the density scaling regime and its implications for the configurational entropy scaling. Scientific Reports, 5(1).

[McCullagh et al., 2005] McCullagh, G. D., Cellai, D., Lawlor, A., and Dawson, K. A. (2005). Finite-energy extension of a lattice glass model. Physical Review E, 71(3).

[McKenna, 2008] McKenna, G. B. (2008). Glass dynamics: Diverging views on glass transition. Nature Physics.

[Montanari and Semerjian, 2006a] Montanari, A. and Semerjian, G. (2006a). Rigorous inequalities between length and time scales in glassy systems. J. Stat. Phys., 125(1):23-54.

[Montanari and Semerjian, 2006b] Montanari, A. and Semerjian, G. (2006b). Rigorous Inequalities Between Length and Time Scales in Glassy Systems. J. Stat. Phys., 125(1):23-54.

[Pauling, 2014] Pauling, L. (2014). General Chemistry (Dover Books on Chemistry). Dover Publications. 
[Pica Ciamarra et al., 2003a] Pica Ciamarra, M., Tarzia, M., de Candia, A., and Coniglio, A. (2003a). Lattice glass model with no tendency to crystallize. Physical Review E, 67(5).

[Pica Ciamarra et al., 2003b] Pica Ciamarra, M., Tarzia, M., de Candia, A., and Coniglio, A. (2003b). Monodisperse model suitable to study the glass transition. Physical Review E, 68(6).

[Poincaré, 1891] Poincaré, H. (1891). Le problème des trois corps. Revue générale des sciences pures et appliquées, 2:1-5.

[Racz, 2002] Racz, Z. (2002). Nonequilibrium phase transitions.

[Ramaswamy, 2010] Ramaswamy, S. (2010). The mechanics and statistics of active matter. Annual Review of Condensed Matter Physics, 1(1):323-345.

[Rapaport, 2004] Rapaport, D. C. (2004). The Art of Molecular Dynamics Simulation. Cambridge University Press.

[Redner et al., 2013] Redner, G. S., Hagan, M. F., and Baskaran, A. (2013). Structure and dynamics of a phase-separating active colloidal fluid. Physical Review Letters, $110(5)$.

[Ritort and Sollich, 2003] Ritort, F. and Sollich, P. (2003). Glassy dynamics of kinetically constrained models. Advances in Physics, 52(4):219-342.

[Rivoire et al., 2004] Rivoire, O., Biroli, G., Martin, O. C., and Mézard, M. (2004). Glass models on bethe lattices. The European Physical Journal B - Condensed Matter and Complex Systems, 37(1):55-78.

[Rovere et al., 1990] Rovere, M., Heermann, D. W., and Binder, K. (1990). The gasliquid transition of the two-dimensional lennard-jones fluid. Journal of Physics: Condensed Matter, 2(33):7009.

[Schweitzer, 2007] Schweitzer, F. (2007). Brownian Agents and Active Particles: Collective Dynamics in the Natural and Social Sciences (Springer Series in Synergetics). Springer.

[Seif and Grigera, 2016] Seif, A. and Grigera, T. S. (2016). Structure and dynamics of the t154 lattice glass.

[Seif et al., 2015] Seif, A., Loscar, E. S., and Grigera, T. S. (2015). Aging and crystallization in a lattice glass model. Physical Review E, 91(4). 
[Sherrington and Kirkpatrick, 1975] Sherrington, D. and Kirkpatrick, S. (1975). Solvable model of a spin-glass. Phys. Rev. Lett., 35:1792-1796.

[Stein and Newman, 2013] Stein, D. and Newman, C. (2013). Spin Glasses and Complexity. Primers in complex systems. Princeton University Press.

[Stillinger and Debenedetti, 2013] Stillinger, F. H. and Debenedetti, P. G. (2013). Glass transition thermodynamics and kinetics. Annual Review of Condensed Matter Physics, 4(1):263-285.

[Strum, 1977] Strum, L. C. E. (1977). Physical aging in plastics and other glassy materials. Polymer Engineering and Science, 17(3):165-173.

[Szabó et al., 2006] Szabó, B., Szöllösi, G. J., Gönci, B., Jurányi, Z., Selmeczi, D., and Vicsek, T. (2006). Phase transition in the collective migration of tissue cells: Experiment and model. Physical Review E, 74(6).

[Thompson et al., 2011] Thompson, A. G., Tailleur, J., Cates, M. E., and Blythe, R. A. (2011). Lattice models of nonequilibrium bacterial dynamics. Journal of Statistical Mechanics: Theory and Experiment, 2011(02):P02029.

[Toner and Tu, 1995] Toner, J. and Tu, Y. (1995). Long-range order in a twodimensional DynamicalXYModel: How birds fly together. Physical Review Letters, 75(23):4326-4329.

[Trefz et al., 2017] Trefz, B., Siebert, J. T., Speck, T., Binder, K., and Virnau, P. (2017). Estimation of the critical behavior in an active colloidal system with vicseklike interactions. The Journal of Chemical Physics, 146(7):074901.

[Varberg et al., 2015] Varberg, J., Gratuze, B., and Kaul, F. (2015). Between egypt, mesopotamia and scandinavia: Late bronze age glass beads found in denmark. Journal of Archaeological Science, 54:168-181.

[Vicsek et al., 1995] Vicsek, T., Czirók, A., Ben-Jacob, E., Cohen, I., and Shochet, O. (1995). Novel type of phase transition in a system of self-driven particles. Physical Review Letters, 75(6):1226-1229.

[Vicsek and Zafeiris, 2012] Vicsek, T. and Zafeiris, A. (2012). Collective motion. Physics Reports, 517(3-4):71-140.

[Voter, 2007] Voter, A. F. (2007). INTRODUCTION TO THE KINETIC MONTE CARLO METHOD, pages 1-23. Springer Netherlands, Dordrecht. 
[Warren and Rottler, 2013] Warren, M. and Rottler, J. (2013). Quench, equilibration, and subaging in structural glasses. Physical Review Letters, 110(2).

[Wu, 1982] Wu, F. Y. (1982). The potts model. Rev. Mod. Phys., 54:235-268.

[Yelash et al., 2008] Yelash, L., Virnau, P., Paul, W., Binder, K., and Müller, M. (2008). Spinodal decomposition of polymer solutions: A parallelized molecular dynamics simulation. Physical Review E, 78(3).

[Zarinelli, 2012] Zarinelli, E. (2012). Spin-glass models and interdisciplinary applications. PhD thesis, Université Paris Sud - Paris XI. 